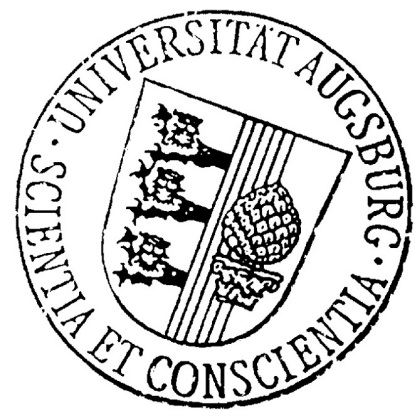

\title{
ATTRACTIVITY AND BIFURCATION FOR NONAUTONOMOUS DYNAMICAL SYSTEMS
}

\author{
Promotionsschrift von \\ Martin Rasmussen \\ Institut für Mathematik \\ Universität Augsburg \\ 86135 Augsburg
}

eingereicht bei der

Mathematisch-Naturwissenschaftlichen Fakultät

der Universität Augsburg

November 2005 
Erstgutachter: Prof. Dr. Fritz Colonius, Universität Augsburg Zweitgutachter: Prof. Dr. Lars Grüne, Universität Bayreuth

Tag der mündlichen Prüfung: 19. April 2006 
To Professor Bernd Aulbach AND MY PARENTS 



\section{CONTENTS}

$\begin{array}{ll}\text { INTRODUCTION } & 3\end{array}$

1 PRELIMINARIES $\quad 9$

$1.1 \quad$ Basic Definitions . . . . . . . . . . . . . . . . . . . 9

1.2 Nonautonomous Dynamical Systems . . . . . . . . . . . . . . . . 10

2 Notions of Attractivity, Repulsivity, BIfURCATION AND Transition 15

2.1 Attractivity and Repulsivity . . . . . . . . . . . . . . . . 15

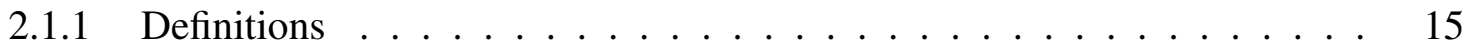

2.1.2 Radii of Attraction and Repulsion . . . . . . . . . . . . . . . 21

2.1.3 Domains of Attraction and Repulsion . . . . . . . . . . . . . 22

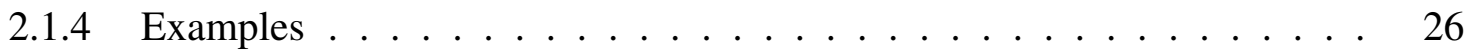

2.1.5 Properties of Time Reversal . . . . . . . . . . . . . . 28

2.1.6 Existence and Uniqueness . . . . . . . . . . . . . . . . . . . . . . 29

2.2 Other Notions of Attractivity and Repulsivity _ . . . . . . . . . . . . . 37

2.2.1 Theory of Stability in the Sense of Lyapunov . . . . . . . . . . . . . . 37

2.2.2 Theory of Autonomous Attractors and Repellers _ . . . . . . . . . . 38

2.2.3 Theory of Nonautonomous Attractors . . . . . . . . . . . . . . 38

2.3 Bifurcation and Transition . . . . . . . . . . . . . . . . 39

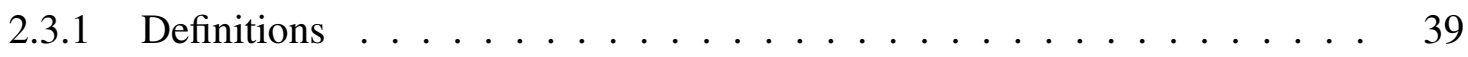

2.3 .2 Examples ............................. 42

2.4 Other Notions of Bifurcation and Transition . . . . . . . . . . . . . . . . . . 44

2.4.1 Autonomous Bifurcation Theory . . . . . . . . . . . . . . . . 45

2.4.2 Bifurcation Theory of Topological Skew Product Flows . . . . . . . . . . 45

2.4.3 Bifurcation Theory of Random Dynamical Systems . . . . . . . . . . . . 46

2.4.4 Bifurcation Theory of General Nonautonomous Dynamical Systems . . . 46 
3 Nonautonomous Morse Decompositions 47

3.1 Nonautonomous Attractor-Repeller Pairs . . . . . . . . . . . . . . . . . . . . . 47

3.2 Nonautonomous Morse Decompositions . . . . . . . . . . . . . . . . 51

3.3 Lyapunov Functions for Nonautonomous Morse Decompositions . . . . . . . . . 55

3.4 Nonautonomous Morse Decompositions in Dimension One . . . . . . . . . . . 57

3.5 Nonautonomous Morse Decompositions of Linear Systems . . . . . . . . . . . . 60

4 LINEAR SYSTEMS $\quad 73$

4.1 Notions of Dichotomy f . . . . . . . . . . . . . 73

4.2 Dichotomy Spectra . . . . . . . . . . . . . . . . 85

4.3 Lyapunov Spectra . . . . . . . . . . . . . . . . . . . . 95

4.4 Spectra of Autonomous Linear Systems _ . . . . . . . . . . . . . . . . . . 97

4.5 Roughness . . . . . . . . . . . . . . . . . . . . . . . 100

5 NONLINEAR SYSTEMS 103

5.1 Nonautonomous Invariant Manifolds . . . . . . . . . . . . . . . . . . . 103

5.2 An Application to Bifurcation Theory . . . . . . . . . . . . . . . . . . 111

5.3 Linearized Attractivity and Repulsivity . . . . . . . . . . . . . . . . . . . 112

5.4 Bifurcation Theory of Adiabatic Systems f . . . . . . . . . . . . . 116

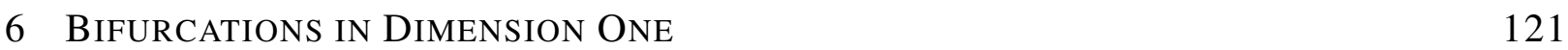

6.1 Nonautonomous Transcritical Bifurcation . . . . . . . . . . . . . . . . 121

6.2 Nonautonomous Pitchfork Bifurcation . . . . . . . . . . . . . . . . . . 127

7 Bifurcations of Asymptotically Autonomous Systems 135

7.1 Basic Properties of Asymptotically Autonomous Systems . . . . . . . . . . . . 136

7.2 Bifurcations in Dimension One . . . . . . . . . . . . . . . . . . 148

7.3 Bifurcations in Dimension Two . . . . . . . . . . . . . . . . . . 160

$\begin{array}{lr}\text { APPENDIX } & 169\end{array}$

A.1 Ordinary Differential Equations . . . . . . . . . . . . . . . . 169

A.2 Useful Lemmata . . . . . . . . . . . . . . . . . . . . . 171

A.3 Projective Spaces . . . . . . . . . . . . . . . . . . . 172

$\begin{array}{ll}\text { BIBLIOGRAPHY } & 175\end{array}$ 


\section{INTRODUCTION}

The mathematical concept of dynamical system is founded on the fact that motions of many application processes are subjected to certain rules. In Newtonian mechanics, in other natural sciences and even in an economical and social context, these laws are given implicitly by a relation that determines the state of a system for all future times just by the knowledge of the present state. A dynamical system therefore consists of the following two components: the space of states and the rule which, given an initial state, allows the projection of the state of the system in the future.

Historically, the notion of dynamical system was derived as an abstraction and generalization of ordinary differential equations. It was first used in 1927 by the American mathematician George D. Birkhoff (1884-1944) in his homonymous book [31]. Birkhoff was strongly influenced by the French mathematician Henri Poincaré (1854-1912), who is regarded-together with the Russian mathematician and engineer Aleksandr M. Lyapunov (1857-1918) —as the father of the so-called qualitative theory of dynamical systems. The goal of the qualitative theory is to understand the behavior of solutions from a more geometrical and topological point of view. In this thesis, we mainly address two aspects of this theory: the theory of attractivity and the theory of bifurcation. These fields are strongly related, since bifurcations from a dynamical viewpoint are associated with loss or gain of attractivity.

The theory of attractivity has its origin in the thesis The General Problem of the Stability of Motion [109, 111, 112], where Lyapunov introduced several definitions and methods to analyze the dynamical behavior in the vicinity of an equilibrium or - more generally — an arbitrary solution of an ordinary differential equation. The term attractor was first used by Coddington and Levinson [47] and Mendelson [118]. In the article Attractors in Dynamical Systems [23], Auslander, Bhatia and Seibert considered attractors consisting of more than one point. In 1967, Stephen Smale introduced in Differential Dynamical Systems [174] a new type of attractor, the axiom A attractor. A new highlight in the theory of attractor was reached in 1971, when Ruelle and Takens regarded so-called strange attractors as a reason for the turbulent behavior in fluids ( $\mathrm{On}$ the $\mathrm{Na}$ ture of Turbulence [147]). This notion of attractor allowed the connection of the attractor theory and the upcoming chaos theory. Similar ideas have formerly been used by Edward N. Lorenz in Deterministic Nonperiodic Flow [107]. In Isolated Invariant Sets and the Morse Index [52], Charles C. Conley introduced in 1978 a very natural notion of local attractor which allowed the construction of so-called attractor-repeller pairs and Morse decompositions, and Ruelle modified this concept by considering so-called pseudoorbits in Small Random Perturbations of Dynamical Systems and the Definition of Attractors [146].

The fundamental ideas and elements of bifurcation theory go back to Poincaré [136] and Lyapunov [110]. Poincaré first used the term bifurcation to describe the splitting of asymptotic states of a dynamical system in his article Sur l'equilibre d'une masse fluids animes d'un mouvement de rotation [134, §2 Equilibre de bifurcation, p. 261]. In 1937, a great step towards a formalization of bifurcation theory was undertaken by the definition of structural stability by Andronov and 
Pontryagin (Systemes grossiers [4]). Since the 1960s, the bifurcation theory was fast-paced. One reason for this development was the introduction of the center manifold theory by Pliss [131] and Kelley [91], which allowed systems of high dimension amenable to a low-dimensional bifurcation analysis. Moreover, the normal form theory, which dates back to the thesis of Poincare [133] and Birkhoff [31], became a field of intensive research.

In many cases, the notion of dynamical system is not general enough to model real world phenomena, since it is often indicated to assume that the underlying rules are time-dependent. For biological processes, for instance, it is more realistic to take evolutionary adaptations into account, and sometimes it is unavoidable to consider random perturbations such as white noise or to model the control of a process by a human being. The appropriate class to treat such problems are the socalled nonautonomous dynamical systems. Another reason to consider nonautonomous dynamical systems is given by the fact that the investigation of states of dynamical systems which are nonconstant in time leads to nonautonomous problems in form of the equation of perturbed motion. The notion of nonautonomous dynamical system was created in the 1990s from the studies of both topological skew product flows and random dynamical systems. The theory of topological skew product flows was founded in the late 1960s by George R. Sell and Richard K. Miller (see $[119,164,165,166])$, and the notion of the random dynamical system is based on research by Baxendale, Bismut, Elworthy, Ikeda, Kunita, Watanabe and many others (see [25, 32, 64, 82, 99]). Further progress in this field was achieved by Ludwig Arnold and his "Bremen Group".

The nonautonomous theory of attractivity has been stimulated in the last fifteen years by the introduction of the notions of pullback attractor, forward attractor, random attractor and weak random attractor. In particular, questions of existence, uniqueness, perturbation and discretization have been addressed. These contributions were made by Cheban, Crauel, Flandoli, Kloeden, Ochs, Schmalfuß and others (see [40, 41, 57, 95, 122]; cf. also Subsection 2.2.3). Nonautonomous bifurcation theory is a new branch which has been developed quite independently for topological skew product flows (see Fabbri, Johnson, Kloeden, Mantellini [66, 84, 85, 86]; cf. also Subsection 2.4.2) and random dynamical systems (see Arnold, Sri Namachchivaya, Schenk-Hoppé $[6,8,155,175]$; cf. also Subsection 2.4.3) so far.

The philosophy behind the present bifurcation theory of nonautonomous dynamical systems is based on a given structure of nonautonomy such as quasi-periodicity or the existence of an invariant measure, and the question arises how to describe bifurcations in a more general nonautonomous context. Recently, Langa, Robinson and Suárez discussed an answer to this question by defining a bifurcation of a nonautonomous differential equation as a merging process of two distinct solutions with different stability behavior (see [102, 104]; cf. also Subsection 2.4.4). In this thesis, other possible approaches are pursued, which are explained demonstratively in the following.

Since the basic understanding of nonautonomous bifur-

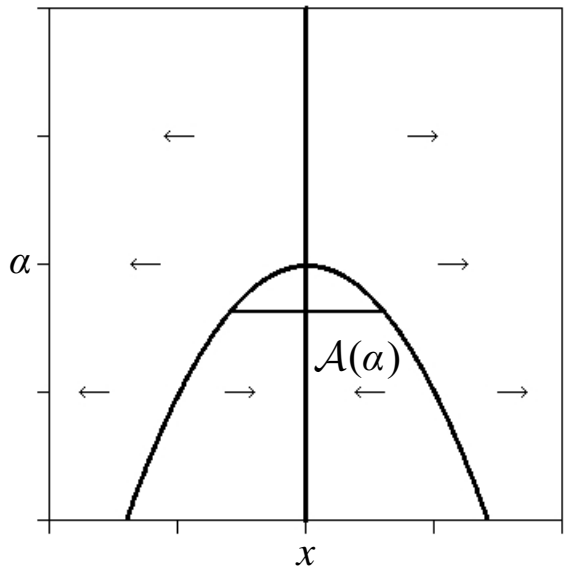

FIGURE 1. Pitchfork bifurcation cations in this thesis is based on phenomenological observations from the autonomous bifurcation theory, it is useful to look exemplarily at an autonomous bifurcation. For a real parameter $\alpha$, consider the ordinary differential equation $\dot{x}=x\left(\alpha+x^{2}\right)$, which is a prototype of a pitchfork bifurcation as indicated in Figure 1. For $\alpha \geq 0$, there is only one equilibrium, which is given 
by zero and which is repulsive. By letting the parameter $\alpha$ pass through zero in negative direction, this equilibrium becomes attractive, and two other repulsive equilibria, given by $\pm \sqrt{-\alpha}$, are bifurcating.

In order to establish a nonautonomous bifurcation theory, consider this scenario in the following way: For $\alpha<0$, the trivial solution is attractive, and the domain of attraction $\mathcal{A}(\alpha)$ is given by the open interval between the two other equilibria. Now, the main point is that this domain of attraction undergoes a qualitative change from a nontrivial to a trivial object in the limit $\alpha \lambda 0$. Moreover, $\mathcal{A}(\alpha)$ is also a repeller, and therefore, also a repeller changes qualitatively for $\alpha \lambda 0$. We call the shrinking of a domain of attraction (repulsion, respectively) a bifurcation, whereas the case of a changing repeller (attractor, respectively) is denoted as a transition.

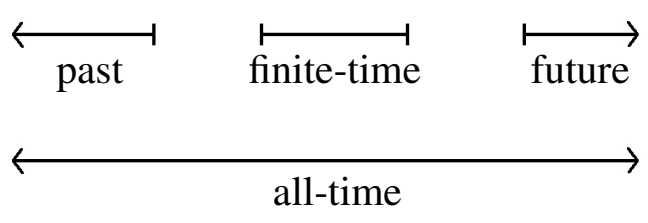

FIGURE 2. Time domains

To implement this idea in the nonautonomous context, locally defined notions of attractive and repulsive solutions, domains of attractivity and repulsivity, as well as attractor and repeller are needed. This thesis distinguishes between four points of view concerning different time domains. The new concepts are introduced for the past (past attractivity, repulsivity, bifurcation and transition), the future (future attractivity, repulsivity, bifurcation and transition), the entire time (all-time attractivity, repulsivity, bifurcation and transition) and compact time intervals (finite-time attractivity, repulsivity, bifurcation and transition) (see Figure 2).

While the first chapter of this thesis is devoted to notational preparations and the introduction of nonautonomous dynamical systems, Chapter 2 contains all relevant notions of attractivity, repulsivity, bifurcation and transition. Several examples illustrate these definitions, and fundamental questions such as existence and uniqueness are discussed. Moreover, the relationship to other notions of attractivity and bifurcation is examined.

Chapter 3 is devoted to Morse decompositions, which were introduced by Charles C. Conley in 1978 to describe the global asymptotic behavior of (autonomous) dynamical systems on compact metric spaces (see [52]). Their components, the so-called Morse sets, are obtained as intersections of attractors and repellers. It is shown that the notions of past and future attractivity and repulsivity are designed to establish nonautonomous generalizations of the Morse decomposition. The dynamical properties of these decompositions are discussed and nonautonomous Lyapunov functions which are constant on the Morse sets are constructed explicitly. Moreover, Morse decompositions of linear systems on the projective space are examined, and a nonautonomous analogon to the Theorem of Selgrade (see [163]) is proved.

In Chapter 4, methods for the analysis of linear systems with respect to the notions of attractivity and repulsivity are introduced. First, several notions of dichotomy are defined, and it is shown that the ranges and null spaces of the corresponding invariant projectors form repellers and attractors of the linear system on the projective space. Furthermore, for the different time domains, dichotomy spectra are introduced which are based on the analysis of the entire time by Sacker and Sell (see [153]) and Siegmund and Aulbach (see [171, 170, 19]). It is also shown that the so-called spectral manifolds give rise to a Morse decomposition on the projective space. This chapter is concluded with a discussion of the relationship to the Lyapunov spectra and some roughness results.

Chapter 5 is devoted to the development of the qualitative theory with respect to the notions of attractivity and repulsivity for nonlinear systems. First, nonautonomous invariant manifolds are constructed, and methods are derived to obtain attractivity and repulsivity from the linearization. 
Moreover, as an application to bifurcation theory, it is shown that the zero is contained in the dichotomy spectrum of a bifurcating solution, and the relationship of the concept of finite-time bifurcation to the bifurcation theory of adiabatic systems is discussed.

The aim of Chapter 6 is to develop counterparts for the classical one-dimensional transcritical and pitchfork bifurcation patterns in the context of nonautonomous bifurcations and transitions. The sufficient conditions are formulated in terms of Taylor coefficients for the right hand side of ordinary differential equations. It is shown that the results are proper generalizations of the autonomous bifurcation scenarios.

In the last chapter of this thesis, asymptotically autonomous systems are discussed. It is supposed that the underlying autonomous system admits a one-dimensional bifurcation of saddle node, pitchfork or transcritical type or a two-dimensional Hopf bifurcation. Sufficient conditions are obtained for the transfer of this bifurcation behavior to the asymptotically autonomous system.

In order to keep this thesis self-contained, some basic facts about ordinary differential equations and projective spaces are noted in the Appendix. Furthermore, the definitions and results are formulated-whenever it was possible - in a very general form. However, to provide reading fluency, attention is restricted to continuous time in Chapter 5, 6 and 7. Extensions for the discrete time can be obtained similarly. Please note that for future reference, the definitions in Chapter 2 are also formulated for noninvertible systems, although invertibility is supposed in all other chapters.

Chapter 1 and 2 are necessary for the understanding of all the other chapters, since they contain both basic facts and the notions of attractivity and bifurcation. The other chapters can be read quite independently of each other. Please note that in Chapter 4, the assertions concerning the Morse decomposition require Chapter 3, and in Chapter 6, results concerning linearized attractivity and repulsivity from Chapter 5 are used.

Finally, please note that - although the applications in this thesis are mainly of low dimensionthe concepts of bifurcation and transition also apply in a higher dimensional setting, since the definitions of attractivity and repulsivity are given in a very general form. The main tool for the analysis of such systems is the method of center manifold reduction (cf. Example 7.2.8). The basic idea is to detect a bifurcation of the system restricted to a center manifold. For instance, consider again the motivating example $\dot{x}=x\left(\alpha+x^{2}\right)$ with an additional second equation, given by $\dot{y}=\lambda y$. In case $\lambda>0$, the trivial solution is not attractive for $\alpha<0$, in contrast to the onedimensional system, and therefore, we have no bifurcation of attraction areas but only a transition of repellers. For $\lambda<0$, the trivial solution is attractive, and thus the two-dimensional system admits a bifurcation of attraction areas, but no longer a repeller transition. Restricting the attention to the lower dimensional invariant manifold $\mathbb{R} \times\{0\}$, however, yields the original one-dimensional system, and for this system we obtain both a bifurcation and a transition.

Writing this dissertation would not have been possible without the aid of many people to whom I would like to express my gratitude. First of all, I would like to thank my supervisor Professor Bernd Aulbach, who unfortunately suddenly and unexpectedly passed away on January 14, 2005, at the age of 57 years. I am grateful for his longstanding support while writing my diploma thesis and dissertation. I benefited from his great ability to explain complicated facts very clearly and lucidly, and I am thankful to him for many fruitful discussions. Moreover, I am greatly indebted to Professor Fritz Colonius who became my advisor after the death of Professor Aulbach. He was very interested in the details of my work, and I was very encouraged by his positive attitude to my ideas and suggestions. I would also like to thank Dr. Stefan Siegmund for many useful discussions and remarks, especially in the first year of my work. Special thanks go to my friends and 
colleagues Dr. Christian Pötzsche and Dr. Ludwig Neidhart for reading the manuscript and making useful comments. I also thank the Deutsche Forschungsgemeinschaft for the financial support I received from them, when I was a member of the Graduiertenkolleg "Nichtlineare Probleme in Analysis, Geometrie und Physik" in the department for mathematics and physics at the University of Augsburg. Finally, I would like to thank my parents for making it possible for me to study mathematics and for their support during all these years.

Augsburg, in November 2005

Martin Rasmussen 



\section{CHAPTER 1}

\section{PRELIMINARIES}

The first section of this chapter is devoted to elementary definitions and notational preparations. In Section 1.2, basic properties of nonautonomous dynamical systems are introduced and discussed.

\subsection{BASIC DEFINITIONS}

As usual, we denote by $\mathbb{Z}$ and $\mathbb{R}$ the sets of all integers and reals, respectively, and we define $\overline{\mathbb{R}}:=\mathbb{R} \cup\{-\infty, \infty\} . \mathbb{R}^{M \times N}$ is the set of all real $M \times N$ matrices, and we write $\mathbb{1}$ for the unit matrix and $\mathbb{O}$ for the zero matrix. Given an arbitrary set $A \subset \mathbb{R}$ and $\kappa \in \mathbb{R}$, we define $A^{ \pm}:=\{x \in \mathbb{R}: x \in A$ or $-x \in A\}, A^{+}:=A \cap(0, \infty), A_{\kappa}^{+}:=A \cap[\kappa, \infty), A^{-}:=A \cap(-\infty, 0)$ and $A_{\kappa}^{-}:=A \cap(-\infty, \kappa]$. Moreover, we set $\mathbb{N}:=\mathbb{Z}^{+}$. For $\mathbb{T}=\mathbb{R}$ or $\mathbb{T}=\mathbb{Z}$, a $\mathbb{T}$-interval is given by the intersection of a real interval with $\mathbb{T}$.

Let $f: X \rightarrow Y$ be a function from a set $X$ to a set $Y$. Then, the graph of $f$ is defined by graph $f:=\{(x, y) \in X \times Y: y=f(x)\}$.

Given a metric space $(X, d)$ and $\varepsilon>0$, we write $U_{\varepsilon}\left(x_{0}\right)=\left\{x \in X: d\left(x, x_{0}\right)<\varepsilon\right\}$ for the $\varepsilon$ neighborhood of a point $x_{0} \in X$ and $U_{\varepsilon}(A)=\cup_{x \in A} U_{\varepsilon}(x)$ for the $\varepsilon$-neighborhood of a set $A \subset X$. The set of all inner points of a nonempty set $A \subset X$ is denoted by int $A$; we write cls $A$ for the closure of $A$ and $\partial A$ for the boundary of $A$. We define the distance of a point $x \in X$ to a nonempty set $A \subset X$ by $d(x, A):=\inf _{y \in A} d(x, y)$ and the Hausdorff semi-distance of two nonempty sets $A, B \subset X$ by

$$
d(A \mid B):=\sup _{x \in A} d(x, B) .
$$

In addition, if both $A$ and $B$ are empty, we set $d(A \mid B):=0$. The Hausdorff distance of $A$ and $B$ is defined by

$$
d_{H}(A, B):=\max \{d(A \mid B), d(B \mid A)\} .
$$

Moreover, for $A, B \subset X$ with $B \subset$ int $A$, we define

$$
\hat{d}(A \mid B):=\sup \left\{r>0: U_{r}(B) \subset A\right\} .
$$

The diameter of a nonempty set $A \subset X$ is given by $\operatorname{diam}(A):=\sup \{d(x, y): x, y \in A\}$. Additionally, we define $\operatorname{diam}(\emptyset):=0$.

If $X$ is a vector space, $A, B \subset X$ and $x \in X$, the following notations will be used:

$$
x+A:=\{x+a: a \in A\} \quad \text { and } \quad A+B:=\{a+b: a \in A, b \in B\} .
$$


With the Euclidian norm

$$
\left\|\left(x_{1}, \ldots, x_{N}\right)\right\|:=\sqrt{\sum_{i=1}^{N} x_{i}^{2}} \text { for all }\left(x_{1}, \ldots, x_{N}\right) \in \mathbb{R}^{N},
$$

induced by the Euclidian scalar product $\langle\cdot, \cdot\rangle$, defined by

$$
\langle x, y\rangle:=\sum_{i=1}^{N} x_{i} y_{i} \quad \text { for all } x=\left(x_{1}, \ldots, x_{N}\right), y=\left(y_{1}, \ldots, y_{N}\right) \in \mathbb{R}^{N},
$$

the $\mathbb{R}^{N}$ is a normed vector space.

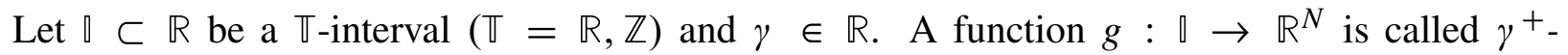

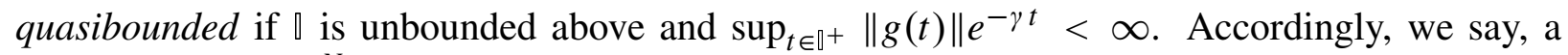

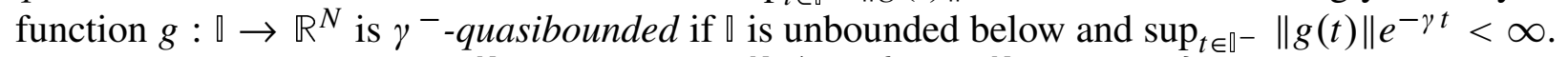
The $(N-1)$-sphere of the $\mathbb{R}^{N}$ is defined by $\mathbb{S}^{N-1}:=\left\{x \in \mathbb{R}^{N}:\|x\|=1\right\}$.

Given a differentiable function $f: X \subset \mathbb{R}^{N} \rightarrow \mathbb{R}^{M}$, we write $D f: X \rightarrow \mathbb{R}^{M \times N}$ for its derivative and $D_{i} f: X \rightarrow \mathbb{R}^{M}$ for its partial derivative with respect to the $i$-th variable, $i \in\{1, \ldots, N\}$. Higher order derivatives $D^{n} f$ or $D_{i}^{n} f$ are defined inductively.

\subsection{Nonautonomous Dynamical Systems}

The notion of nonautonomous dynamical system has emerged in the late 1990s as an abstraction of both continuous skew product flows (see, e.g., Miller [119] and SELL [164, 165, 166]) and random dynamical systems (see, e.g., the monograph ARNOLD [5]). The definition is given as follows (see also the conference proceedings Colonius \& KLOEDEN \& SiEgmund [51]).

1.2.1 Definition (Nonautonomous Dynamical System). A (local) nonautonomous dynamical system (NDS for short) on a metric space $X$ with a time $\mathbb{T}$ $\left(=\mathbb{R}, \mathbb{R}_{0}^{+}, \mathbb{Z}, \mathbb{Z}_{0}^{+}\right)$and base set $P$ is a pair of mappings

$$
\left(\theta: \mathbb{T}^{ \pm} \times P \rightarrow P, \varphi: D \subset \mathbb{T} \times P \times X \rightarrow X\right)
$$

with the following properties:

(i) The so-called base flow or driving system $\theta$ is a dynamical system, i.e., we have the relations

$$
\theta(0, p)=p \quad \text { and } \quad \theta(t+s, p)=\theta(t, \theta(s, p)) \quad \text { for all } p \in P \text { and } t, s \in \mathbb{T}^{ \pm} .
$$

(ii) The maximal interval of existence $D_{\max }(p, x):=\{t \in \mathbb{T}:(t, p, x) \in D\}$ for $p \in P$ and $x \in X$ is either empty or an open $\mathbb{T}$-interval which contains $0 \in \mathbb{T}$.

(iii) $\varphi$ is a cocycle over $\theta$, i.e., for all $t, s \in \mathbb{T}$ and $(p, x) \in P \times X$ with $s \in D_{\max }(p, x)$ and $t+s \in D_{\max }(p, x)$, we have $t \in D_{\max }(\theta(s, p), \varphi(s, p, x))$ and

$$
\varphi(0, p, x)=x \quad \text { and } \quad \varphi(t+s, p, x)=\varphi(t, \theta(s, p), \varphi(s, p, x)) .
$$

(iv) $\varphi$ is continuous with respect to $t \in \mathbb{T}$ and $x \in X$. 
$X$ is called phase space, and $P \times X$ is called extended phase space. We say, a nonautonomous dynamical system is invertible if $\mathbb{T}=\mathbb{R}$ or $\mathbb{T}=\mathbb{Z}$ and $t \in D_{\max }(p, x)$ for some $p \in P$ and $x \in X$ implies $-t \in D_{\max }(\theta(t, p), \varphi(t, p, x))$.

For simplicity in notation, we also write $\theta_{t} p$ instead of $\theta(t, p)$ and $\varphi(t, p) x$ for $\varphi(t, p, x)$.

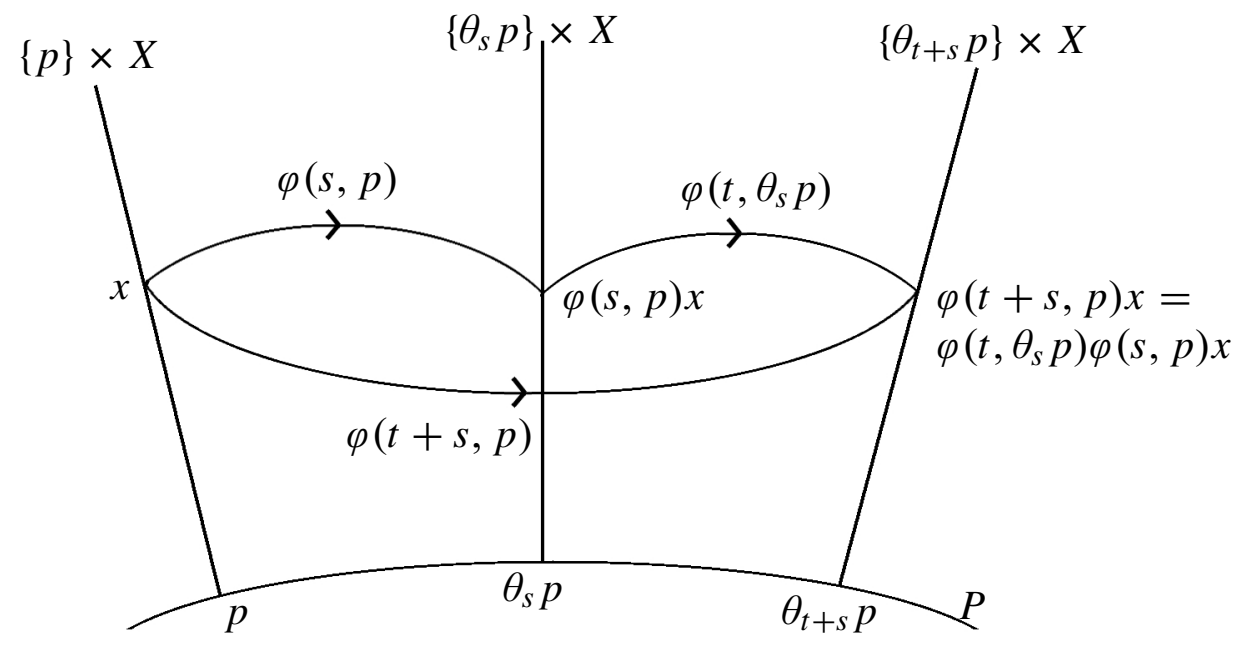

FIGURE 1.1. Property (iii) of Definition 1.2.1.

A standard example of a nonautonomous dynamical system, which is of main interest in this thesis, is provided by a nonautonomous ordinary differential equation

$$
\dot{x}=f(t, x)
$$

with $f: D \subset \mathbb{R} \times \mathbb{R}^{N} \rightarrow \mathbb{R}^{N}$ (see Appendix A.1). Here, $\mathbb{T}=\mathbb{R}$, and the base set $P$ can simply be chosen to be $\mathbb{R}$ with base flow $(t, s) \mapsto t+s$. In case the function $f$ is fulfilling special conditions, the nonautonomous differential equation (1.1) gives rise to a general solution $\lambda: \Omega \subset \mathbb{R} \times \mathbb{R} \times \mathbb{R}^{N} \rightarrow \mathbb{R}^{N}$ (see Proposition A.1.3), and $\varphi$ can then be defined by

$$
\varphi(t, s) x:=\lambda(t+s, s, x) \text { for all }(t, s, x) \in \mathbb{R} \times \mathbb{R} \times \mathbb{R}^{N} \text { such that }(t+s, s, x) \in \Omega .
$$

Without further notice, we assume that all ordinary differential equations considered in this thesis fulfill conditions of local existence and uniqueness of solutions.

A similar construction is also possible for nonautonomous difference equations of the form $x_{n+1}=f\left(n, x_{n}\right), \mathbb{T}=P=\mathbb{Z}$.

In both cases above, however, $P$ is noncompact, which may cause difficulties. This can be avoided for a special class of right hand sides $f$ by considering the Bebutov flow on the hull of $f$ (see, e.g., Bebutov [26] and Sell [166]).

Apart from deterministic also random and stochastic differential and difference equations (see, e.g., ARNOLD [5]) and some other types of equations such as functional differential equations or nonautonomous evolutionary equations generate nonautonomous dynamical systems.

\subsubsection{REMARKS.}

(i) Normally, one has additional structures concerning the driving system $\theta$. In the deterministic case, the base set $P$ is a metric space and $\theta$ is continuous; in case of random dynamical systems, $\theta$ represents an ergodic dynamical system. For a discussion of the relationship of these two concepts, see BERGER \& SIEGMUND [28]. 
(ii) In the literature, one usually considers global nonautonomous dynamical systems, i.e., $D=\mathbb{T} \times P \times X$. The above definition of a local nonautonomous dynamical system stems from AulbaCH \& SiEgmund \& RASMUSSEN [16] (see also RASMUSSEN [143]; in case of random dynamical systems, see ARNOLD [5] and ARNOLD \& NAMACHCHIVAYA \& SCHENK-HOPPÉ [8]).

(iii) Although using the definition of a local nonautonomous dynamical system, we try to avoid overloaded notation. For instance, in Definition 2.1.1 (iii), the statement "for all $\varepsilon>0, \ldots$, we have $\varphi\left(t, \theta_{-t} p\right) U_{\varepsilon}\left(R\left(\theta_{-t} p\right)\right) \supset M(p)$ " means actually "there exists an $\hat{\varepsilon}>0$ such that for all $\varepsilon \in(0, \hat{\varepsilon}), \ldots$, we have $\varphi\left(t, \theta_{-t} p\right) U_{\varepsilon}\left(R\left(\theta_{-t} p\right)\right) \supset M(p)$ ". Here, the situation for small $\varepsilon$ is crucial, and writing the "abbreviated form" does not imply that $\varphi\left(t, \theta_{-t} p, \cdot\right)$ is globally defined.

Let $\theta$ be a base flow on $P$. For an element $p \in P$, we define the forward orbit of $p$ by $\mathcal{O}^{+}(p):=$ $\left\{\theta_{t} p: t \geq 0\right\}$, the backward orbit of $p$ by $\mathcal{O}^{-}(p):=\left\{\theta_{t} p: t \leq 0\right\}$, the $T$-orbit of $p, T \in \mathbb{T}^{+}$, by $\mathcal{O}^{T}(p):=\left\{\theta_{t} p: t \in[0, T] \cap \mathbb{T}\right\}$ and the orbit of $p$ by $\mathcal{O}(p):=\left\{\theta_{t} p: t \in \mathbb{T}^{ \pm}\right\}$. Two elements $p_{1}, p_{2} \in P$ are called equivalent $\left(p_{1} \sim p_{2}\right)$ if $p_{1} \in \mathcal{O}\left(p_{2}\right)$. We denote the set of all equivalence classes $[p]$ by $P / \sim$.

1.2.3 DeFinition (NonAutonomous Sets). We consider a nonautonomous dynamical system $(\theta, \varphi)$ on a metric space $X$ with a base set $P$. For an arbitrary set $M \subset P \times X$, we define the so-called $p$-fibre of $M$ by

$$
M(p):=\{x \in X:(p, x) \in M\} \text { for all } p \in P,
$$

and we denote by $P^{*}(M):=\{p \in P: M(p) \neq \emptyset\}$ the set of all base elements leading to nonempty fibres. $M$ is called

(i) past nonautonomous set if $\mathcal{O}^{-}(p) \subset P^{*}(M)$ for all $p \in P^{*}(M)$,

(ii) future nonautonomous set if $\mathcal{O}^{+}(p) \subset P^{*}(M)$ for all $p \in P^{*}(M)$,

(iii) all-time nonautonomous set if $\mathcal{O}(p) \subset P^{*}(M)$ for all $p \in P^{*}(M)$,

(iv) $(p, T)$-nonautonomous set if $\mathcal{O}^{T}(p) \subset P^{*}(M)$.

We say that $M$ is

(i) invariant if $\varphi(t, p) M(p)=M\left(\theta_{t} p\right)$ for all $p \in P^{*}(M)$ and $t \in \mathbb{T}$ with $\theta_{t} p \in P^{*}(M)$,

(ii) closed if $M(p)$ is closed for all $p \in P^{*}(M)$,

(iii) compact if $M(p)$ is compact for all $p \in P^{*}(M)$.

\subsubsection{REMARKS.}

(i) An all-time nonautonomous set is a past, as well as a future nonautonomous set. The reversal is certainly not true.

(ii) In the literature, an all-time nonautonomous set $M$ with $P^{*}(M)=P$ is called nonautonomous set.

1.2.5 Proposition. Let $(\theta, \varphi)$ be a NDS with a locally compact phase space $X$ and $M$ be a compact and invariant past (future, all-time, $(p, T)$-, respectively) nonautonomous set. Then, 
for all $p \in P^{*}(M), \varepsilon>0$ and $t \in \mathbb{T}$ with $\theta_{t} p \in P^{*}(M)$, there exists a $\delta>0$ with

$$
\varphi(t, p) U_{\delta}(M(p)) \subset U_{\varepsilon}\left(M\left(\theta_{t} p\right)\right)
$$

Proof. Given $p \in P^{*}(M)$, due to the local compactness, there exists a $\beta>0$ such that cls $U_{\beta}(M(p))$ is compact. Let $\varepsilon>0$ and $t \in \mathbb{T}$ with $\theta_{t} p \in P^{*}(M)$. Then, the map $\varphi(t, p, \cdot)$ is uniformly continuous on the compact set cls $U_{\beta}(M(p))$. Thus, there exists a $\delta \in(0, \beta)$ with $\varphi(t, p) U_{\delta}(M(p)) \subset U_{\varepsilon}\left(M\left(\theta_{t} p\right)\right)$.

The following definition is adapted from AUBIN \& FRANKOWSKA [12] (see also AKIN [3, Exercise 1.5, p. 9] and Elstrodt [63, p. 9]).

1.2.6 Definition. For a past nonautonomous set $M \subset P \times X$ and $p \in P^{*}(M)$, we define $\limsup _{t \rightarrow \infty} M\left(\theta_{-t} p\right):=\bigcap_{\tau \geq 0} \bigcup_{t \geq \tau} M\left(\theta_{-t} p\right) \quad$ and $\quad \liminf _{t \rightarrow \infty} M\left(\theta_{-t} p\right):=\bigcup_{\tau \geq 0} \bigcap_{t \geq \tau} M\left(\theta_{-t} p\right)$.

Given a future nonautonomous set $M \subset P \times X$ and $p \in P^{*}(M)$, we define

$$
\limsup _{t \rightarrow \infty} M\left(\theta_{t} p\right):=\bigcap_{\tau \geq 0} \bigcup_{t \geq \tau} M\left(\theta_{t} p\right) \quad \text { and } \quad \liminf _{t \rightarrow \infty} M\left(\theta_{t} p\right):=\bigcup_{\tau \geq 0} \bigcap_{t \geq \tau} M\left(\theta_{t} p\right) \text {. }
$$

It is easy to show that the following characterizations hold:

- $\lim \sup _{t \rightarrow \infty} M\left(\theta_{-t} p\right)=\left\{x \in X: \forall \tau \geq 0: \exists t \geq \tau: x \in M\left(\theta_{-t} p\right)\right\}$,

- $\liminf _{t \rightarrow \infty} M\left(\theta_{-t} p\right)=\left\{x \in X: \exists \tau \geq 0: \forall t \geq \tau: x \in M\left(\theta_{-t} p\right)\right\}$,

- $\lim \sup _{t \rightarrow \infty} M\left(\theta_{t} p\right)=\left\{x \in X: \forall \tau \geq 0: \exists t \geq \tau: x \in M\left(\theta_{t} p\right)\right\}$,

- $\liminf _{t \rightarrow \infty} M\left(\theta_{t} p\right)=\left\{x \in X: \exists \tau \geq 0: \forall t \geq \tau: x \in M\left(\theta_{t} p\right)\right\}$. 



\section{CHAPTER 2}

\section{NOTIONS OF ATTRACTIVITY, REPULSIVITY, BIFURCATION AND TRANSITION}

In this chapter, new concepts of (local) attractivity and repulsivity (in Section 2.1) and bifurcation and transition (in Section 2.3) are introduced for nonautonomous dynamical systems. By a bifurcation and transition, a qualitative change of the attractivity or repulsivity of nonautonomous sets is meant. Due to the nonautonomous framework, it is distinguished between four distinct points of view concerning different time domains. The notions of attractivity and repulsivity-and for this reason also the notions of bifurcation and transition - are introduced for the past (past attractivity and repulsivity), the future (future attractivity and repulsivity), the entire time (all-time attractivity and repulsivity) and the present (finite-time attractivity and repulsivity) of the system.

Since the definitions in this chapter are new to a broad extent, the relationship to well-known concepts is discussed in Section 2.2 (in case of attractivity and repulsivity) and Section 2.4 (in case of bifurcation and transition).

\subsection{ATTRACTIVITY AND REPULSIVITY}

This section is divided into six subsections. In the first subsection, several notions of nonautonomous attractor and repeller are introduced, and equivalent characterizations in special situations are formulated. In Subsection 2.1.2 and 2.1.3, the theoretical background to analyze the strength of attractivity and repulsivity is established. The introduced definitions are illustrated by means of two examples in Subsection 2.1.4, and in Subsection 2.1.5, properties of the definitions under time reversal are studied. In the last subsection, criteria for the existence of attractors and repellers are formulated, and the question of their uniqueness is discussed.

Throughout this section, let $\left(\theta: \mathbb{T}^{ \pm} \times P \rightarrow P, \varphi: D \subset \mathbb{T} \times P \times X \rightarrow X\right)$ be a nonautonomous dynamical system with an arbitrary base set $P$ and a metric space $(X, d)$.

2.1.1 Definitions. We begin with the definitions concerning the past of the system.

2.1.1 Definition (PASt Attractivity And Repulsivity). Let $A$ and $R$ be invariant and compact past nonautonomous sets and $\mathcal{M}$ be a collection of past nonautonomous sets. 
(i) $A$ is called $\mathcal{M}$-past attractor if for all $M \in \mathcal{M}$, we have $P^{*}(M) \subset P^{*}(A)$ and

$$
\lim _{t \rightarrow \infty} d\left(\varphi\left(t, \theta_{-t} p\right) M\left(\theta_{-t} p\right) \mid A(p)\right)=0 \quad \text { for all } p \in P^{*}(M) .
$$

(ii) $A$ is called past attractor if $A$ is an $\{M\}$-past attractor for some past nonautonomous set $M$ fulfilling the following property: There exists an $\eta>0$ such that for all $p \in P^{*}(A)$, there exists a $\hat{p} \in[p] \cap P^{*}(A)$ with

$$
U_{\eta}\left(A\left(\theta_{-t} \hat{p}\right)\right) \subset M\left(\theta_{-t} \hat{p}\right) \quad \text { for all } t \geq 0 .
$$

(iii) $R$ is called $\mathcal{M}$-past repeller if for all $M \in \mathcal{M}$, we have $P^{*}(M) \subset P^{*}(R)$, and for all $M \in \mathcal{M}, p \in P^{*}(M)$ and $\varepsilon>0$, there exists a $t^{*}>0$ with

$$
\varphi\left(t, \theta_{-t} p\right) U_{\varepsilon}\left(R\left(\theta_{-t} p\right)\right) \supset M(p) \text { for all } t \geq t^{*} .
$$

(iv) $R$ is called past repeller if $R$ is an $\{M\}$-past repeller for some past nonautonomous set $M$ fulfilling the following property: There exists an $\eta>0$ such that for all $p \in P^{*}(R)$, there exists a $\hat{p} \in[p] \cap P^{*}(R)$ with

$$
U_{\eta}\left(R\left(\theta_{-t} \hat{p}\right)\right) \subset M\left(\theta_{-t} \hat{p}\right) \quad \text { for all } t \geq 0 .
$$

It follows directly from the definitions that the empty set is both a past attractor and a past repeller. If $X$ is compact and $D=\mathbb{T} \times P \times X$, then $P \times X$ is also both a past attractor and a past repeller.

To understand Definition 2.1.1, the following equivalent characterizations are useful.

2.1.2 Proposition (Equivalent Characterizations). Let $A$ and $R$ be invariant and compact past nonautonomous sets and $\mathcal{M}$ be a collection of past nonautonomous sets. Then, the following statements are fulfilled:

(i) In case $X$ is locally compact, $A$ is an $\mathcal{M}$-past attractor if and only if for all $M \in \mathcal{M}$, we have $P^{*}(M) \subset P^{*}(A)$, and for all $M \in \mathcal{M}$ and $p \in P^{*}(M)$, there exists a $\hat{p} \in[p] \cap P^{*}(M)$ with

$$
\lim _{t \rightarrow \infty} d\left(\varphi\left(t, \theta_{-t} \hat{p}\right) M\left(\theta_{-t} \hat{p}\right) \mid A(\hat{p})\right)=0
$$

(ii) $A$ is a past attractor if and only if there exists an $\eta>0$ such that for all $p \in P^{*}(A)$, there exists a $\hat{p} \in[p] \cap P^{*}(A)$ with

$$
\lim _{t \rightarrow \infty} d\left(\varphi\left(t, \theta_{-\tau-t} \hat{p}\right) U_{\eta}\left(A\left(\theta_{-\tau-t} \hat{p}\right)\right) \mid A\left(\theta_{-\tau} \hat{p}\right)\right)=0 \quad \text { for all } \tau \geq 0 .
$$

(iii) In case $X$ is locally compact, $A$ is a past attractor if and only if there exists an $\eta>0$ such that for all $p \in P^{*}(A)$, we have

$$
\lim _{t \rightarrow \infty} d\left(\varphi\left(t, \theta_{-t} p\right) U_{\eta}\left(A\left(\theta_{-t} p\right)\right) \mid A(p)\right)=0 .
$$

(iv) In case $X$ is locally compact, $A$ is a past attractor if and only if there exists an $\eta>0$ such that for all $p \in P^{*}(A)$, there exists a $\hat{p} \in[p] \cap P^{*}(A)$ with 


$$
\lim _{t \rightarrow \infty} d\left(\varphi\left(t, \theta_{-t} \hat{p}\right) U_{\eta}\left(A\left(\theta_{-t} \hat{p}\right)\right) \mid A(\hat{p})\right)=0 .
$$

(v) $R$ is a past repeller if and only if there exists an $\eta>0$ such that for all $p \in P^{*}(R)$, there exists a $\hat{p} \in[p] \cap P^{*}(R)$ such that for all $\varepsilon>0$ and $\tau \geq 0$, we have a $t^{*}>0$ with

$$
\varphi\left(t, \theta_{-\tau-t} \hat{p}\right) U_{\varepsilon}\left(R\left(\theta_{-\tau-t} \hat{p}\right)\right) \supset U_{\eta}\left(R\left(\theta_{-\tau} \hat{p}\right)\right) \quad \text { for all } t \geq t^{*} .
$$

In addition, we assume that $(\theta, \varphi)$ is invertible. Then, the following statements are fulfilled:

(vi) $R$ is an $\mathcal{M}$-past repeller if and only if for all $M \in \mathcal{M}$, we have $P^{*}(M) \subset P^{*}(R)$ and

$$
\lim _{t \rightarrow \infty} d\left(\varphi(-t, p) M(p) \mid R\left(\theta_{-t} p\right)\right)=0 \quad \text { for all } p \in P^{*}(M) .
$$

(vii) $R$ is a past repeller if and only if there exists an $\eta>0$ such that for all $p \in P^{*}(R)$, there exists a $\hat{p} \in[p] \cap P^{*}(R)$ with

$$
\lim _{t \rightarrow \infty} d\left(\varphi\left(-t, \theta_{-\tau} \hat{p}\right) U_{\eta}\left(R\left(\theta_{-\tau} \hat{p}\right)\right) \mid R\left(\theta_{-\tau-t} \hat{p}\right)\right)=0 \quad \text { for all } \tau \geq 0
$$

Proof. (ii), (v), (vi) and (vii) are direct consequences of Definition 2.1.1. (i), (iii) and (iv) follow using Proposition 1.2.5.

\subsubsection{REMARKS.}

(i) The notions of Definition 2.1.1 represent the behavior of $(\theta, \varphi)$ in the past. This can be seen by considering another nonautonomous dynamical system $(\theta, \bar{\varphi}: D \subset \mathbb{T} \times P \times X \rightarrow X)$ with the following property: For all $\bar{p} \in P$, there exists a $\hat{p} \in[\bar{p}]$ with

$$
\varphi(t, p) x=\bar{\varphi}(t, p) x \quad \text { for all }(t, p, x) \in D \text { fulfilling } p, \theta_{t} p \in \mathcal{O}^{-}(\hat{p})
$$

Then, for any past attractor (repeller, respectively) $A$ of $(\theta, \varphi)$, there exists a past attractor (repeller, respectively) $\bar{A}$ of $(\theta, \bar{\varphi})$ such that for all $\bar{p} \in P$, there exists a $\hat{p} \in[\bar{p}]$ with

$$
A(p)=\bar{A}(p) \text { for all } p \in \mathcal{O}^{-}(\hat{p}) .
$$

(ii) Let $A$ be a past attractor with $\eta$ given as in Definition 2.1.1 (ii). In case $X$ is locally compact, for all $p \in P^{*}(A)$, we have

$$
A(p)=\limsup _{t \rightarrow \infty} \varphi\left(t, \theta_{-t} p\right) U_{\eta}\left(A\left(\theta_{-t} p\right)\right)=\liminf _{t \rightarrow \infty} \varphi\left(t, \theta_{-t} p\right) U_{\eta}\left(A\left(\theta_{-t} p\right)\right) .
$$

In the following definition, the notions of future attractivity and repulsivity are explained.

2.1.4 Definition (Future Attractivity and Repulsivity). Let $A$ and $R$ be invariant and compact future nonautonomous sets and $\mathcal{M}$ be a collection of future nonautonomous sets.

(i) $A$ is called $\mathcal{M}$-future attractor if for all $M \in \mathcal{M}$, we have $P^{*}(M) \subset P^{*}(A)$ and

$$
\lim _{t \rightarrow \infty} d\left(\varphi(t, p) M(p) \mid A\left(\theta_{t} p\right)\right)=0 \quad \text { for all } p \in P^{*}(M) .
$$


(ii) $A$ is called future attractor if $A$ is an $\{M\}$-future attractor for some future nonautonomous set $M$ fulfilling the following property: There exists an $\eta>0$ such that for all $p \in P^{*}(A)$, there exists a $\hat{p} \in[p] \cap P^{*}(A)$ with

$$
U_{\eta}\left(A\left(\theta_{t} \hat{p}\right)\right) \subset M\left(\theta_{t} \hat{p}\right) \quad \text { for all } t \geq 0 \text {. }
$$

(iii) $R$ is called $\mathcal{M}$-future repeller if for all $M \in \mathcal{M}$, we have $P^{*}(M) \subset P^{*}(R)$, and for all $M \in \mathcal{M}, p \in P^{*}(M)$ and $\varepsilon>0$, there exists a $t^{*}>0$ with

$$
\varphi(t, p) U_{\varepsilon}(R(p)) \supset M\left(\theta_{t} p\right) \quad \text { for all } t \geq t^{*} .
$$

(iv) $R$ is called future repeller if $R$ is an $\{M\}$-future repeller for some future nonautonomous set $M$ fulfilling the following property: There exists an $\eta>0$ such that for all $p \in P^{*}(R)$, there exists a $\hat{p} \in[p] \cap P^{*}(R)$ with

$$
U_{\eta}\left(R\left(\theta_{t} \hat{p}\right)\right) \subset M\left(\theta_{t} \hat{p}\right) \quad \text { for all } t \geq 0 \text {. }
$$

It follows directly from the definitions that the empty set is both a future attractor and future repeller. If $X$ is compact and $D=\mathbb{T} \times P \times X$, then $P \times X$ is also a future attractor and future repeller.

2.1.5 Proposition (Equivalent Characterizations). Let $A$ and $R$ be invariant and compact future nonautonomous sets and $\mathcal{M}$ be a collection of future nonautonomous sets. Then, the following statements are fulfilled:

(i) $A$ is a future attractor if and only if there exists an $\eta>0$ such that for all $p \in P^{*}(A)$, there exists a $\hat{p} \in[p] \cap P^{*}(A)$ with

$$
\lim _{t \rightarrow \infty} d\left(\varphi\left(t, \theta_{\tau} \hat{p}\right) U_{\eta}\left(A\left(\theta_{\tau} \hat{p}\right)\right) \mid A\left(\theta_{\tau+t} \hat{p}\right)\right)=0 \quad \text { for all } \tau \geq 0 .
$$

(ii) $R$ is a future repeller if and only if there exists an $\eta>0$ such that for all $p \in P^{*}(R)$, there exists a $\hat{p} \in[p] \cap P^{*}(R)$ such that for all $\varepsilon>0$ and $\tau \geq 0$, we have a $t^{*}>0$ with

$$
\varphi\left(t, \theta_{\tau} \hat{p}\right) U_{\varepsilon}\left(R\left(\theta_{\tau} \hat{p}\right)\right) \supset U_{\eta}\left(R\left(\theta_{\tau+t} \hat{p}\right)\right) \quad \text { for all } t \geq t^{*} .
$$

In addition, we assume that $(\theta, \varphi)$ is invertible. Then, the following statements are fulfilled:

(iii) $R$ is an $\mathcal{M}$-future repeller if and only if for all $M \in \mathcal{M}$, we have $P^{*}(M) \subset P^{*}(R)$ and

$$
\lim _{t \rightarrow \infty} d\left(\varphi\left(-t, \theta_{t} p\right) M\left(\theta_{t} p\right) \mid R(p)\right)=0 \quad \text { for all } p \in P^{*}(M) .
$$

(iv) In case $X$ is locally compact, $R$ is an $\mathcal{M}$-future repeller if and only if for all $M \in \mathcal{M}$, we have $P^{*}(M) \subset P^{*}(R)$, and for all $M \in \mathcal{M}$ and $p \in P^{*}(M)$, there exists a $\hat{p} \in[p] \cap P^{*}(M)$ with

$$
\lim _{t \rightarrow \infty} d\left(\varphi\left(-t, \theta_{t} \hat{p}\right) M\left(\theta_{t} \hat{p}\right) \mid R(\hat{p})\right)=0 .
$$

(v) $R$ is a future repeller if and only if there exists an $\eta>0$ such that for all $p \in P^{*}(R)$, 
there exists a $\hat{p} \in[p] \cap P^{*}(R)$ with

$$
\lim _{t \rightarrow \infty} d\left(\varphi\left(-t, \theta_{\tau+t} \hat{p}\right) U_{\eta}\left(R\left(\theta_{\tau+t} \hat{p}\right)\right) \mid R\left(\theta_{\tau} \hat{p}\right)\right)=0 \quad \text { for all } \tau \geq 0 .
$$

(vi) In case $X$ is locally compact, $R$ is a future repeller if and only if there exists an $\eta>0$ such that for all $p \in P^{*}(R)$, we have

$$
\lim _{t \rightarrow \infty} d\left(\varphi\left(-t, \theta_{t} p\right) U_{\eta}\left(R\left(\theta_{t} p\right)\right) \mid R(p)\right)=0 .
$$

(vii) In case $X$ is locally compact, $R$ is a future repeller if and only if there exists an $\eta>0$ such that for all $p \in P^{*}(R)$, there exists a $\hat{p} \in[p] \cap P^{*}(R)$ with

$$
\lim _{t \rightarrow \infty} d\left(\varphi\left(-t, \theta_{t} \hat{p}\right) U_{\eta}\left(R\left(\theta_{t} \hat{p}\right)\right) \mid R(\hat{p})\right)=0
$$

PROOF. See proof of Proposition 2.1.2.

\subsubsection{REMARKS.}

(i) As seen in Remark 2.1.3 (i) in case of past attractivity and repulsivity, the notions of Definition 2.1.4 represent the behavior of $(\theta, \varphi)$ in the future.

(ii) There are important analogies in the concepts of past and future attractivity and repulsivity, but in the general noninvertible case, it is not possible to reduce the amount of definitions. For the invertible case, this question is treated in Section 2.1.5. It is shown that a past attractor corresponds to a future repeller, and a past repeller is related to a future attractor.

(iii) Suppose, $(\theta, \varphi)$ is invertible and $X$ is locally compact, and let $R$ be a future repeller with $\eta$ given as in Definition 2.1.4 (iv). Then, for all $p \in P^{*}(R)$, we have

$$
R(p)=\limsup _{t \rightarrow \infty} \varphi\left(-t, \theta_{t} p\right) U_{\eta}\left(R\left(\theta_{t} p\right)\right)=\liminf _{t \rightarrow \infty} \varphi\left(-t, \theta_{t} p\right) U_{\eta}\left(R\left(\theta_{t} p\right)\right) .
$$

In the following definition, the notions of all-time attractivity and repulsivity are explained.

2.1.7 Definition (All-Time Attractivity And Repulsivity). Let $A$ and $R$ be invariant and compact all-time nonautonomous sets.

(i) $A$ is called all-time attractor if there exists an $\eta>0$ with

$$
\lim _{t \rightarrow \infty} \sup _{p \in P^{*}(A)} d\left(\varphi(t, p) U_{\eta}(A(p)) \mid A\left(\theta_{t} p\right)\right)=0 .
$$

(ii) $R$ is called all-time repeller if there exists an $\eta>0$ such that for all $\varepsilon>0$, there exists a $t^{*}>0$ with

$$
\varphi(t, p) U_{\varepsilon}(R(p)) \supset U_{\eta}\left(R\left(\theta_{t} p\right)\right) \quad \text { for all } p \in P^{*}(R) \text { and } t \geq t^{*} .
$$

It follows directly from the definitions that the empty set is both an all-time attractor and an alltime repeller. If $X$ is compact and $D=\mathbb{T} \times P \times X$, then $P \times X$ is also an all-time attractor and all-time repeller. An all-time attractor (all-time repeller, respectively) is also both a past attractor 
(past repeller, respectively) and a future attractor (future repeller, respectively), since in the above definition, $p$ can be replaced by $\theta_{-t} p$.

In case of invertibility, Definition 2.1.7 (ii) can be simplified.

2.1.8 Proposition (Equivalent Characterization). Let $R$ be an invariant and compact all-time nonautonomous set, and suppose, $(\theta, \varphi)$ is invertible. Then, $R$ is an alltime repeller if and only if there exists an $\eta>0$ with

$$
\lim _{t \rightarrow \infty} \sup _{p \in P^{*}(R)} d\left(\varphi(-t, p) U_{\eta}(R(p)) \mid R\left(\theta_{-t} p\right)\right)=0 .
$$

Finally, the definitions of finite-time attractivity and repulsivity are introduced.

2.1.9 Definition (Finite-Time Attractivity And Repulsivity). For $p \in P$ and $T>0$, let $A$ and $R$ be invariant and compact $(p, T)$-nonautonomous sets.

(i) $A$ is called $(p, T)$-attractor if

$$
\underset{\eta \unlhd 0}{\limsup } \frac{1}{\eta} d\left(\varphi(T, p) U_{\eta}(A(p)) \mid A\left(\theta_{T} p\right)\right)<1 .
$$

(ii) $R$ is called $(p, T)$-repeller if

$$
\liminf _{\eta \unlhd 0} \frac{1}{\eta} \hat{d}\left(\varphi(T, p) U_{\eta}(R(p)) \mid R\left(\theta_{T} p\right)\right)>1 .
$$

2.1.10 REMARK. In contrast to the above definitions in case of past, future and all-time attractivity and repulsivity, the notions of finite-time attractivity and repulsivity are not invariant with respect to a change of the metric to an equivalent metric.

In case of invertibility, Definition 2.1.9 (ii) can be simplified.

2.1.11 Proposition (Equivalent Characterization). Let $R$ be an invariant and compact $(p, T)$-nonautonomous set. If $(\theta, \varphi)$ is invertible, then $R$ is a $(p, T)$-repeller if and only if

$$
\underset{\eta \triangleleft 0}{\limsup } \frac{1}{\eta} d\left(\varphi\left(-T, \theta_{T} p\right) U_{\eta}\left(R\left(\theta_{T} p\right)\right) \mid R(p)\right)<1 .
$$

We will often consider invariant nonautonomous sets which are solutions of the nonautonomous dynamical system $(\theta, \varphi)$.

2.1.12 Definition (Solution). Let $\hat{P} \subset P$ be nonempty with $\hat{P} \subset \mathcal{O}(p)$ for some $p \in P$. A function $\mu: \hat{P} \rightarrow X$ is called solution of $(\theta, \varphi)$ if graph $\mu$ is invariant.

The attractivity and repulsivity of solutions are defined by considering the graph of the solution as an invariant nonautonomous set. 
2.1.13 Definition (Attractivity and Repulsivity of Solutions). Let $\mu$ be a solution of $(\theta, \varphi)$.

(i) $\mu$ is called past attractive (future attractive, all-time attractive, $(p, T)$-attractive, respectively) if $\operatorname{graph}(\mu)$ is a past attractor (future attractor, all-time attractor, $(p, T)$-attractor, respectively).

(ii) $\mu$ is called past repulsive (future repulsive, all-time repulsive $(p, T)$-repulsive, respectively) if $\operatorname{graph}(\mu)$ is a past repeller (future repeller, all-time repeller, $(p, T)$-repeller, respectively).

Concluding this subsection, we state the following proposition, whose obvious proof will be omitted.

2.1.14 Proposition. A past nonautonomous (future nonautonomous, all-time nonautonomous, $(p, T)$-nonautonomous, respectively) set can never be both a past attractor (future attractor, all-time attractor, $(p, T)$-attractor, respectively) and a past repeller (future repeller, all-time repeller, $(p, T)$-repeller, respectively).

2.1.2 Radii of Attraction and Repulsion. Since the local dynamical behavior of nonautonomous sets is studied in the definitions of the preceding subsection, it is useful to know something about the range of attractivity or repulsivity. In this subsection, notions of radii of attraction and repulsion are introduced.

2.1.15 Definition (RAdi OF ATTRACTION AND REPUlsion). We define the radius of past attraction of a past attractor $A$ by

$$
\begin{aligned}
\mathfrak{A}_{A}^{\leftarrow}:=\sup \{\eta>0: & \text { For all } p \in P^{*}(A), \text { there exists a } \hat{p} \in[p] \cap P^{*}(A) \text { with } \\
& \left.\lim _{t \rightarrow \infty} d\left(\varphi\left(t, \theta_{-\tau-t} \hat{p}\right) U_{\eta}\left(A\left(\theta_{-\tau-t} \hat{p}\right)\right) \mid A\left(\theta_{-\tau} \hat{p}\right)\right)=0 \text { for all } \tau \geq 0\right\}
\end{aligned}
$$

(cf. also Proposition 2.1.2 (ii)) and the radius of past repulsion of a past repeller $R$ by

$$
\begin{aligned}
\mathfrak{R}_{R}^{\leftarrow}:=\sup \{\eta>0: & \text { For all } p \in P^{*}(R), \text { there exists a } \hat{p} \in[p] \cap P^{*}(R) \text { such that for all } \\
& \varepsilon>0 \text { and } \tau \geq 0, \text { we have a } t^{*}>0 \text { with } \\
& \left.\varphi\left(t, \theta_{-\tau-t} \hat{p}\right) U_{\varepsilon}\left(R\left(\theta_{-\tau-t} \hat{p}\right)\right) \supset U_{\eta}\left(R\left(\theta_{-\tau} \hat{p}\right)\right) \text { for all } t \geq t^{*}\right\}
\end{aligned}
$$

(cf. also Proposition 2.1.2(v)). The radius of future attraction of a future attractor $A$ is defined by

$$
\begin{aligned}
\mathfrak{R}_{A}:=\sup \{\eta>0: & \text { For all } p \in P^{*}(A), \text { there exists a } \hat{p} \in[p] \cap P^{*}(A) \text { with } \\
& \left.\lim _{t \rightarrow \infty} d\left(\varphi\left(t, \theta_{\tau} \hat{p}\right) U_{\eta}\left(A\left(\theta_{\tau} \hat{p}\right)\right) \mid A\left(\theta_{t+\tau} \hat{p}\right)\right)=0 \text { for all } \tau \geq 0\right\}
\end{aligned}
$$

(cf. also Proposition 2.1.5 (i)), and the radius of future repulsion of a future repeller $R$ is defined by 


$$
\begin{aligned}
\Re_{R}:=\sup \{\eta>0: & \text { For all } p \in P^{*}(R), \text { there exists a } \hat{p} \in[p] \cap P^{*}(R) \text { such that } \\
& \text { for all } \varepsilon>0 \text { and } \tau \geq 0, \text { we have a } t^{*}>0 \text { with } \\
& \left.\varphi\left(t, \theta_{\tau} \hat{p}\right) U_{\varepsilon}\left(R\left(\theta_{\tau} \hat{p}\right)\right) \supset U_{\eta}\left(R\left(\theta_{\tau+t} \hat{p}\right)\right) \text { for all } t \geq t^{*}\right\}
\end{aligned}
$$

(cf. also Proposition 2.1.5 (ii)). The radius of all-time attraction of an all-time attractor $A$ is defined by

$$
\mathfrak{R}_{A}^{\leftrightarrow}:=\sup \left\{\eta>0: \lim _{t \rightarrow \infty} \sup _{p \in P^{*}(A)} d\left(\varphi(t, p) U_{\eta}(A(p)) \mid A\left(\theta_{t} p\right)\right)=0\right\}
$$

(cf. also Definition 2.1.7 (i)), and the radius of all-time repulsion of an all-time repeller $R$ is defined by

$$
\begin{aligned}
\mathfrak{R}_{R}^{\leftrightarrow}:=\sup \{\eta>0: & \text { For all } \varepsilon>0, \text { there exists a } t^{*}>0 \text { with } \\
& \left.\varphi(t, p) U_{\varepsilon}(R(p)) \supset U_{\eta}\left(R\left(\theta_{t} p\right)\right) \text { for all } p \in P^{*}(R) \text { and } t \geq t^{*}\right\}
\end{aligned}
$$

(cf. also Definition 2.1.7 (ii)). The radius of $(p, T)$-attraction of a $(p, T)$-attractor $A$ is defined by

$$
\mathfrak{A}_{A}^{(p, T)}:=\sup \left\{\eta>0: d\left(\varphi(T, p) U_{\hat{\eta}}(A(p)) \mid A\left(\theta_{T} p\right)\right)<\hat{\eta} \text { for all } \hat{\eta} \in(0, \eta)\right\}
$$

(cf. also Definition 2.1.9 (i)), and the radius of $(p, T)$-repulsion of a $(p, T)$-repeller $R$ is defined by

$$
\mathfrak{R}_{R}^{(p, T)}:=\sup \left\{\eta>0: \hat{d}\left(\varphi(T, p) U_{\hat{\eta}}(R(p)) \mid R\left(\theta_{T} p\right)\right)>\hat{\eta} \text { for all } \hat{\eta} \in(0, \eta)\right\}
$$

(cf. also Definition 2.1.9 (ii)).

When considering a solution $\mu$ of $(\theta, \varphi)$ which is either past (future, all-time, $(p, T)$-, respectively) attractive or repulsive, one of the above definitions applies for graph $\mu$. We write $\mathfrak{A}_{\mu}:=\mathfrak{A}_{\text {graph } \mu}$ or $\mathfrak{R}_{\mu}:=\mathfrak{R}_{\text {graph } \mu}$ and proceed similarly with further notation (concerning, e.g., the domains of attraction and repulsion introduced in the next subsection).

2.1.3 Domains of Attraction and Repulsion. The above defined radii of attraction and repulsion are positive real numbers. However, if $X$ is a finite-dimensional Banach space (or equivalently locally compact, see LANG [101, Corollary 3.15, p. 39]) and in case of past, future and all-time attractivity and repulsivity, we will — in addition to the radii of attraction and repulsion-consider domains of attraction and repulsion as subsets of the phase space.

We begin with some auxiliary definitions for the extended phase space. Given a past attractor $A$, we define for all $p \in P^{*}(A)$

$$
\begin{aligned}
\mathcal{A}_{A}^{\leftarrow}(p):=\{x \in X: & \text { There exists a neighborhood } U \text { of } x \text { such that } \\
& \left.\lim _{t \rightarrow \infty} d\left(\varphi\left(t, \theta_{-t} p\right)\left(A\left(\theta_{-t} p\right)+U\right) \mid A(p)\right)=0\right\}
\end{aligned}
$$


(cf. also Proposition 2.1.2 (iii)), and for a past repeller $R$, we define for all $p \in P^{*}(R)$

$$
\begin{aligned}
\mathcal{R}_{R}^{\leftarrow}(p):=\{x \in X: & \text { There exists a neighborhood } U \text { of } x \text { such that } \\
& \text { for all } \varepsilon>0, \text { there exists a } t^{*}>0 \text { with } \\
& \left.\varphi\left(t, \theta_{-t} p\right) U_{\varepsilon}\left(R\left(\theta_{-t} p\right)\right) \supset R(p)+U \text { for all } t \geq t^{*}\right\}
\end{aligned}
$$

(cf. also Proposition 2.1.2 (v)). In case the $\operatorname{NDS}(\theta, \varphi)$ is invertible, for all $p \in P^{*}(R)$, we have

$$
\begin{array}{r}
\mathcal{R}_{R}^{\leftarrow}(p)=\{x \in X: \text { There exists a neighborhood } U \text { of } x \text { such that } \\
\left.\qquad \lim _{t \rightarrow \infty} d\left(\varphi(-t, p)(R(p)+U) \mid R\left(\theta_{-t} p\right)\right)=0\right\}
\end{array}
$$

(cf. also Proposition 2.1.2 (vii)).

Given a future attractor $A$, we define for all $p \in P^{*}(A)$

$$
\begin{aligned}
\mathcal{A}_{A}(p):=\{x \in X: & \text { There exists a neighborhood } U \text { of } x \text { such that } \\
& \left.\lim _{t \rightarrow \infty} d\left(\varphi(t, p)(A(p)+U) \mid A\left(\theta_{t} p\right)\right)=0\right\}
\end{aligned}
$$

(cf. also Proposition 2.1.5 (i)), and for a future repeller $R$, we define for all $p \in P^{*}(R)$

$\mathcal{R}_{R}(p):=\{x \in X:$ There exists a neighborhood $U$ of $x$ such that for all $\varepsilon>0$, there exists a $t^{*}>0$ with $\varphi(t, p) U_{\varepsilon}(R(p)) \supset R\left(\theta_{t} p\right)+U$ for all $\left.t \geq t^{*}\right\}$

(cf. also Proposition 2.1.5 (ii)). In case the $\operatorname{NDS}(\theta, \varphi)$ is invertible, for all $p \in P^{*}(R)$, we have

$$
\begin{aligned}
\mathcal{R}_{R}(p)=\{x \in X: & \text { There exists a neighborhood } U \text { of } x \text { such that } \\
& \left.\lim _{t \rightarrow \infty} d\left(\varphi\left(-t, \theta_{t} p\right)\left(R\left(\theta_{t} p\right)+U\right) \mid R(p)\right)=0\right\}
\end{aligned}
$$

(cf. also Proposition 2.1.5 (vi)).

Some properties of these sets are derived in the following proposition.

2.1.16 Proposition. Suppose, $X$ is a finite-dimensional Banach space. Then, the following statements are fulfilled:

(i) Given a past attractor $A$, for all $p \in P^{*}(A)$, the set $\mathcal{A}_{A}^{\leftarrow}(p)$ is open, and we have $\mathcal{A}_{A}^{\leftarrow}(p)=\mathcal{A}_{A}^{\leftarrow}(\hat{p})$ for all $\hat{p} \in[p] \cap P^{*}(A)$. Furthermore, for all $p \in P^{*}(A)$ and compact sets $C \subset \mathcal{A}_{A}^{\leftarrow}(p)$, the relation

$$
\lim _{t \rightarrow \infty} d\left(\varphi\left(t, \theta_{-t} p\right)\left(A\left(\theta_{-t} p\right)+C\right) \mid A(p)\right)=0
$$

is fulfilled.

(ii) Given a past repeller $R$, for all $p \in P^{*}(R)$, the set $\mathcal{R}_{R}^{\leftarrow}(p)$ is open. Furthermore, for all $p \in P^{*}(R)$, compact sets $C \subset \mathcal{R}_{A}^{\leftarrow}(p)$ and $\varepsilon>0$, there exists a $t^{*}>0$ with

$$
\varphi\left(t, \theta_{-t} p\right) U_{\varepsilon}\left(R\left(\theta_{-t} p\right)\right) \supset R(p)+C \quad \text { for all } t \geq t^{*} .
$$

In case of invertibility, the last formula is equivalent to

$$
\lim _{t \rightarrow \infty} d\left(\varphi(-t, p)(R(p)+C) \mid R\left(\theta_{-t} p\right)\right)=0 .
$$


(iii) Given a future attractor $A$, for all $p \in P^{*}(A)$, the set $\mathcal{A}_{A}(p)$ is open. Furthermore, for all $p \in P^{*}(A)$ and compact sets $C \subset \mathcal{A}_{A}(p)$, the relation

$$
\lim _{t \rightarrow \infty} d\left(\varphi(t, p)(A(p)+C) \mid A\left(\theta_{t} p\right)\right)=0
$$

is fulfilled.

(iv) In addition, we suppose that the $\operatorname{NDS}(\theta, \varphi)$ is invertible. Then, given a future repeller $R$, for all $p \in P^{*}(R)$, the set $\mathcal{R}_{R}(p)$ is open, and we have $\mathcal{R}_{R}(p)=\mathcal{R}_{R}(\hat{p})$ for all $\hat{p} \in[p] \cap P^{*}(R)$. Furthermore, for all $p \in P^{*}(R)$ and compact sets $C \subset \mathcal{R}_{R}(p)$, the relation

$$
\lim _{t \rightarrow \infty} d\left(\varphi\left(-t, \theta_{t} p\right)\left(R\left(\theta_{t} p\right)+C\right) \mid R(p)\right)=0
$$

is fulfilled.

Proof. (i) The openness of $\mathcal{A}_{A}^{\leftarrow}(p)$ is a direct consequence of its definition, and the second assertion follows from Proposition 1.2.5, since the finite-dimensional Banach space $X$ is locally compact (see LANG [101, Corollary 3.15, p. 39]). Let us now assume that there exist $p \in P^{*}(A)$, a compact set $C \subset \mathcal{A}_{A}^{\leftarrow}(p)$, an $\varepsilon>0$ and sequences $\left\{x_{n}\right\}_{n \in \mathbb{N}}$ in $C$ and $\left\{t_{n}\right\}_{n \in \mathbb{N}}$ in $\mathbb{T}$ such that $\lim _{n \rightarrow \infty} t_{n}=\infty$ and

$$
d\left(\varphi\left(t_{n}, \theta_{-t_{n}} p\right)\left(A\left(\theta_{-t_{n}} p\right)+x_{n}\right) \mid A(p)\right) \geq \varepsilon \quad \text { for all } n \in \mathbb{N} .
$$

Since $C$ is compact, we assume w.l.o.g. that $\left\{x_{n}\right\}_{n \in \mathbb{N}}$ is convergent with $\lim _{n \rightarrow \infty} x_{n}=x_{0}$. Since $x_{0} \in C \subset \mathcal{A}_{A}^{\leftarrow}(p)$, there exists a neighborhood $U$ of $x_{0}$ such that

$$
\lim _{t \rightarrow \infty} d\left(\varphi\left(t, \theta_{-t} p\right)\left(A\left(\theta_{-t} p\right)+U\right) \mid A(p)\right)=0 .
$$

This is a contradiction.

(ii) As in (i), the first assertion is clear. Suppose now, there exist a $p \in P^{*}(R)$, a compact set $C \subset \mathcal{R}_{R}^{\leftarrow}(p)$, an $\varepsilon>0$ and sequences $\left\{x_{n}\right\}_{n \in \mathbb{N}}$ in $C$ and $\left\{t_{n}\right\}_{n \in \mathbb{N}}$ in $\mathbb{T}$ such that $\lim _{n \rightarrow \infty} t_{n}=\infty$ and

$$
\varphi\left(t_{n}, \theta_{-t_{n}} p\right) U_{\varepsilon}\left(R\left(\theta_{-t_{n}} p\right)\right) \not \supset R(p)+x_{n} \quad \text { for all } n \in \mathbb{N} .
$$

Since $C$ is compact, we assume w.l.o.g. that $\left\{x_{n}\right\}_{n \in \mathbb{N}}$ is convergent with $\lim _{n \rightarrow \infty} x_{n}=x_{0}$. Since $x_{0} \in C \subset \mathcal{R}_{R}^{\leftarrow}(p)$, there exists a neighborhood $U$ of $x_{0}$ such that for all $\varepsilon>0$, there exists a $t^{*}>0$ with

$$
\varphi\left(t, \theta_{-t} p\right) U_{\varepsilon}\left(R\left(\theta_{-t} p\right)\right) \supset R(p)+U \text { for all } t \geq t^{*} .
$$

This is a contradiction and finishes the proof of (ii).

The proofs of (iii) and (iv) will be omitted, since they are similar to (i) and (ii).

For simplicity in description, it is our aim to characterize the strength of attractivity or repulsivity not by the above defined fiber-wise sets, but by subsets of the phase space. This reduction is done by the following definition.

2.1.17 Definition (Domains of Attraction And Repulsion). Suppose, $X$ is a finite-dimensional Banach space.

(i) The domain of past attraction of a past attractor $A$ is defined by 


$$
\mathcal{A}_{A}^{\leftarrow}:=\bigcap_{p \in P^{*}(A)} \liminf _{t \rightarrow \infty} \mathcal{A}_{A}^{\leftarrow}\left(\theta_{-t} p\right)
$$

(ii) The domain of past repulsion of a past repeller $R$ is defined by

$$
\mathcal{R}_{R}^{\leftarrow}:=\bigcap_{p \in P^{*}(R)} \liminf _{t \rightarrow \infty} \mathcal{R}_{R}^{\leftarrow}\left(\theta_{-t} p\right)
$$

(iii) The domain of future attraction of a future attractor $A$ is defined by

$$
\mathcal{A}_{A}:=\bigcap_{p \in P^{*}(A)} \liminf _{t \rightarrow \infty} \mathcal{A}_{A}\left(\theta_{t} p\right)
$$

(iv) The domain of future repulsion of a future repeller $R$ is defined by

$$
\mathcal{R}_{R}:=\bigcap_{p \in P^{*}(R)} \liminf _{t \rightarrow \infty} \mathcal{R}_{R}\left(\theta_{t} p\right) .
$$

(v) The domain of all-time attraction of an all-time attractor $A$ is defined by

$$
\begin{aligned}
\mathcal{A}_{A}^{\leftrightarrow}:=\{x \in X: & \text { There exists a neighborhood } U \text { of } x \\
& \text { such that } \left.\lim _{t \rightarrow \infty} \sup _{p \in P^{*}(A)} d\left(\varphi(t, p)(A(p)+U) \mid A\left(\theta_{t} p\right)\right)=0\right\} .
\end{aligned}
$$

(vi) The domain of all-time repulsion of an all-time repeller $R$ is defined by

$$
\begin{aligned}
\mathcal{R}_{R}^{\leftrightarrow}:=\{x \in X: & \text { There exists a neighborhood } U \text { of } x \text { such that } \\
& \text { for all } \varepsilon>0 \text {, there exists a } t^{*}>0 \text { with } \\
& \left.\varphi(t, p) U_{\varepsilon}(R(p)) \supset R\left(\theta_{t} p\right)+U \text { for all } p \in P^{*}(R) \text { and } t \geq t^{*}\right\} .
\end{aligned}
$$

In case the $\operatorname{NDS}(\theta, \varphi)$ is invertible, we have

$$
\begin{aligned}
& \mathcal{R}_{R}^{\leftrightarrow}=\{x \in X: \text { There exists a neighborhood } U \text { of } x \text { such that } \\
&\left.\qquad \lim _{t \rightarrow \infty} \sup _{p \in P^{*}(R)} d\left(\varphi(-t, p)(R(p)+U) \mid R\left(\theta_{-t} p\right)\right)=0\right\} .
\end{aligned}
$$

\subsubsection{REMARKS.}

(i) It can be seen immediately from the definitions that all above defined domains of attraction and repulsion are neighborhoods of zero.

(ii) The relations

$$
\begin{aligned}
& \mathfrak{A}_{A}^{\leftarrow}=\hat{d}\left(\mathcal{A}_{A}^{\leftarrow} \mid\{0\}\right), \quad \mathfrak{A}_{A}=\hat{d}\left(\mathcal{A}_{A} \mid\{0\}\right), \quad \mathfrak{R}_{R}=\hat{d}\left(\mathcal{R}_{R} \mid\{0\}\right) \quad \text { and } \\
& \mathfrak{R}_{R}^{\leftarrow}=\hat{d}\left(\mathcal{R}_{R}^{\leftarrow} \mid\{0\}\right), \quad \mathfrak{A}_{A}^{\leftrightarrow}=\hat{d}\left(\mathcal{A}_{A}^{\leftrightarrow} \mid\{0\}\right), \quad \mathfrak{R}_{R}^{\leftrightarrow}=\hat{d}\left(\mathcal{R}_{R}^{\leftrightarrow} \mid\{0\}\right)
\end{aligned}
$$

are fulfilled. 
(iii) Given a past attractor $A$, from Proposition 2.1.16 (i), the relation

$$
\mathcal{A}_{A}^{\leftarrow}=\bigcap_{p \in P^{*}(A)} \mathcal{A}_{A}^{\leftarrow}(p)
$$

follows. In case of invertibility, Proposition 2.1.16 (iv) implies for a future repeller $R$,

$$
\mathcal{R}_{R}=\bigcap_{p \in P^{*}(R)} \mathcal{R}_{R}(p) .
$$

2.1.19 Proposition. Suppose, $X$ is a finite-dimensional Banach space and $(\theta, \varphi)$ is invertible. Then, the following statements are fulfilled:

(i) Let $\mu: \mathcal{O}^{-}(p) \rightarrow X$ be a past repulsive solution. Then, the past nonautonomous set with the $\theta_{-t} p$-fibres $\mathcal{R}_{\mu}^{\leftarrow}\left(\theta_{-t} p\right)+\mu\left(\theta_{-t} p\right), t \geq 0$, is invariant.

(ii) Let $\mu: \mathcal{O}^{+}(p) \rightarrow X$ be a future attractive solution. Then, the future nonautonomous set with the $\theta_{t} p$-fibres $\mathcal{A}_{\mu}\left(\theta_{t} p\right)+\mu\left(\theta_{t} p\right), t \geq 0$, is invariant.

Proof. (i) We choose $\tau \in \mathbb{T}_{0}^{+}, \hat{\tau} \in \mathbb{T} \cap(-\infty, \tau]$ and $x \in \mathcal{R}_{\mu}^{\leftarrow}\left(\theta_{-\tau} p\right)+\mu\left(\theta_{-\tau} p\right)$. Let $U$ be a neighborhood of $x-\mu\left(\theta_{-\tau} p\right)$ such that

$$
\lim _{t \rightarrow \infty} d\left(\varphi\left(-t, \theta_{-\tau} p\right)\left(\mu\left(\theta_{-\tau} p\right)+U\right) \mid\left\{\mu\left(\theta_{-\tau-t} p\right)\right\}\right)=0 .
$$

Since $(\theta, \varphi)$ is invertible, the set $\varphi\left(\hat{\tau}, \theta_{-\tau} p\right)\left(\mu\left(\theta_{-\tau} p\right)+U\right)$ is a neighborhood of $\varphi\left(\hat{\tau}, \theta_{-\tau} p\right) x$, and we have

$$
\begin{aligned}
& \left.\lim _{t \rightarrow \infty} d\left(\varphi\left(-t, \theta_{\hat{\tau}-\tau} p\right) \varphi\left(\hat{\tau}, \theta_{-\tau} p\right)\left(\mu\left(\theta_{-\tau} p\right)+U\right)\right) \mid\left\{\mu\left(\theta_{\hat{\tau}-\tau-t} p\right)\right\}\right) \\
= & \lim _{t \rightarrow \infty} d\left(\varphi\left(-t, \theta_{-\tau} p\right)\left(\mu\left(\theta_{-\tau} p\right)+U\right) \mid\left\{\mu\left(\theta_{-\tau-t}\right)\right\}\right) \stackrel{(2.1)}{=} 0 .
\end{aligned}
$$

This means that $\varphi\left(\hat{\tau}, \theta_{-\tau} p\right) x-\mu\left(\theta_{\hat{\tau}-\tau}\right) \in \mathcal{R}_{\mu}^{\leftarrow}\left(\theta_{\hat{\tau}-\tau} p\right)$

The assertion (ii) can be proved analogously.

2.1.4 Examples. In this subsection, two examples-given by a linear and a nonlinear onedimensional differential equation - are discussed in order to illustrate the notions of attractivity and repulsivity introduced in the preceding three subsections.

2.1.20 EXAMPLE. We consider the linear nonautonomous differential equation

$$
\dot{x}=a(t) x
$$

with a continuous function $a: \mathbb{R} \rightarrow \mathbb{R}$, which generates a nonautonomous dynamical system with $\mathbb{T}=P=\mathbb{R}$ (see Section 1.2). It is easy to see that every invariant and compact all-time nonautonomous set $M \subset \mathbb{R} \times \mathbb{R}$ is a

- past attractor with $\mathcal{A}_{M}^{\leftarrow}=\mathbb{R}$ if and only if $\lim _{t \rightarrow-\infty} \int_{t}^{0} a(s) d s=-\infty$

- past repeller with $\mathcal{R}_{M}^{\leftarrow}=\mathbb{R}$ if and only if $\lim _{t \rightarrow-\infty} \int_{t}^{0} a(s) d s=\infty$ 
- future attractor with $\mathcal{A}_{M}=\mathbb{R}$ if and only if $\lim _{t \rightarrow \infty} \int_{0}^{t} a(s) d s=-\infty$,

- future repeller with $\mathcal{R}_{M}=\mathbb{R}$ if and only if $\lim _{t \rightarrow \infty} \int_{0}^{t} a(s) d s=\infty$,

- all-time attractor with $\mathcal{A}_{M}^{\leftrightarrow}=\mathbb{R}$ if and only if $\lim _{t \rightarrow \infty} \sup _{\tau \in \mathbb{R}} \int_{\tau}^{\tau+t} a(s) d s=-\infty$,

- all-time repeller with $\mathcal{R}_{M}^{\leftrightarrow}=\mathbb{R}$ if and only if $\lim _{t \rightarrow \infty} \sup _{\tau \in \mathbb{R}} \int_{\tau}^{\tau+t} a(s) d s=\infty$,

- $(p, T)$-attractor with $\mathfrak{A}_{M}^{(p, T)}=\infty$ if and only if $\int_{p}^{p+T} a(s) d s<0$,

- $(p, T)$-repeller with $\mathfrak{R}_{M}^{(p, T)}=\infty$ if and only if $\int_{p}^{p+T} a(s) d s>0$.

2.1.21 EXAMPLE. The nonautonomous differential equation

$$
\dot{x}=a(t) x+b(t) x^{3}=x\left(a(t)+b(t) x^{2}\right)
$$

with continuous functions $a: \mathbb{R} \rightarrow \mathbb{R}$ and $b: \mathbb{R} \rightarrow \mathbb{R}_{\kappa}^{+}$for some $\kappa>0$ generates a nonautonomous dynamical system with $\mathbb{T}=P=\mathbb{R}$ (see Section 1.2). For simplicity, we define

$$
w(t):=\sqrt{-\frac{a(t)}{b(t)}} \text { for all } t \in \mathbb{R} \text { with } a(t)<0 .
$$

Then, for fixed $t \in \mathbb{R}$ with $a(t)<0$, the zero set of the right hand side is $\{0, \pm w(t)\}$; for all $t \in \mathbb{R}$ with $a(t) \geq 0$, this zero set is the singleton $\{0\}$. An elementary discussion of the sign of the right hand side of this equation yields that the trivial solution is

- past attractive with

$$
\left(-\liminf _{t \rightarrow-\infty} w(t), \liminf _{t \rightarrow-\infty} w(t)\right) \subset \mathcal{A}_{0}^{\leftarrow} \subset\left(-\limsup _{t \rightarrow-\infty} w(t), \limsup _{t \rightarrow-\infty} w(t)\right)
$$

if $\liminf _{t \rightarrow-\infty}-\frac{a(t)}{b(t)}>0$,

- past repulsive with $\mathcal{R}_{0}^{\leftarrow}=\mathbb{R}$ if $\lim \sup _{t \rightarrow-\infty}-\frac{a(t)}{b(t)} \leq 0$,

- future attractive with

$$
\left(-\liminf _{t \rightarrow \infty} w(t), \liminf _{t \rightarrow \infty} w(t)\right) \subset \mathcal{A}_{0} \subset\left(-\limsup _{t \rightarrow \infty} w(t), \limsup _{t \rightarrow \infty} w(t)\right)
$$

if $\liminf _{t \rightarrow \infty}-\frac{a(t)}{b(t)}>0$,

- future repulsive with $\mathcal{R}_{0}=\mathbb{R}$ if $\lim \sup _{t \rightarrow \infty}-\frac{a(t)}{b(t)} \leq 0$,

- all-time attractive with

$$
\left(-\inf _{t \in \mathbb{R}} w(t), \inf _{t \in \mathbb{R}} w(t)\right) \subset \mathcal{A}_{0}^{\leftrightarrow} \subset\left(-\sup _{t \in \mathbb{R}} w(t), \sup _{t \in \mathbb{R}} w(t)\right)
$$

if $\inf _{t \in \mathbb{R}}-\frac{a(t)}{b(t)}>0$,

- all-time repulsive with $\mathcal{R}_{0}^{\leftrightarrow}=\mathbb{R}$ if $-\frac{a(t)}{b(t)} \leq 0$ for all $t \in \mathbb{R}$,

- $(p, T)$-attractive with

$$
\inf _{t \in[p, p+T]} w(t) \leq \mathfrak{A}_{0}^{(p, T)} \leq \sup _{t \in[p, p+T]} w(t)
$$

if $-\frac{a(t)}{b(t)}>0$ for all $t \in[p, p+T]$, 
- $(p, T)$-repulsive with $\mathfrak{R}_{0}^{(p, T)}=\infty$ if $-\frac{a(t)}{b(t)} \leq 0$ for all $t \in[p, p+T]$.

These conditions are only sufficient for attractivity or repulsivity of the trivial solution but not necessary.

2.1.5 Properties of Time Reversal. As the reader may have observed, there are important analogies in the concepts of, say, past repulsivity and future attractivity. The aim of this subsection is to study these relationships.

We assume that $(\theta, \varphi)$ is invertible, which implies $\mathbb{T}=\mathbb{R}$ or $\mathbb{T}=\mathbb{Z}$, and we consider the NDS under time reversal, denoted by $\left(\theta^{-1}, \varphi^{-1}\right)$ and defined by the relations

$$
\begin{aligned}
\theta^{-1}(t, p) & :=\theta(-t, p) & & \text { for all }(t, p) \in \mathbb{T} \times P, \\
\varphi^{-1}(t, p, x) & :=\varphi(-t, p, x) & & \text { for all }(t, p, x) \text { with }(-t, p, x) \in D .
\end{aligned}
$$

The pair $(\theta, \varphi)^{-1}:=\left(\theta^{-1}, \varphi^{-1}\right)$ is indeed a nonautonomous dynamical system, since we have

$$
\begin{aligned}
\varphi^{-1}(t+s, p, x) & =\varphi(-t-s, p, x)=\varphi(-t, \theta(-s, p), \varphi(-s, p, x)) \\
& =\varphi^{-1}\left(t, \theta^{-1}(s, p), \varphi^{-1}(s, p, x)\right)
\end{aligned}
$$

for all $t, s \in \mathbb{T}$ and $(p, x) \in P \times X$ with $-s \in D_{\max }(p, x)$ and $-t-s \in D_{\max }(p, x)$.

2.1.22 Proposition (Properties of Time Reversal). Let $M$ be a subset of $P \times X$. Then, the following statements are fulfilled:

(i) $M$ is a past attractor of $(\theta, \varphi)$ if and only if $M$ is a future repeller of $(\theta, \varphi)^{-1}$. We have $\mathfrak{A}_{M}^{\leftarrow}=\mathfrak{R}_{M}$. If, in addition, $X$ is a finite-dimensional Banach space, then also $\mathcal{A}_{M}^{\overleftarrow{M}}=\mathcal{R}_{M} \rightarrow$ is fulfilled

(ii) $M$ is a past repeller of $(\theta, \varphi)$ if and only if $M$ is a future attractor of $(\theta, \varphi)^{-1}$. We have $\mathfrak{R}_{M}^{\leftarrow}=\mathfrak{A}_{M}$. If, in addition, $X$ is a finite-dimensional Banach space, then also $\mathcal{R}_{M}^{\leftarrow}=\mathcal{A}_{M}$ is fulfilled

(iii) $M$ is an all-time attractor of $(\theta, \varphi)$ if and only if $M$ is an all-time repeller of $(\theta, \varphi)^{-1}$. We have $\mathfrak{R}_{M}^{\leftrightarrow}=\mathfrak{A}_{M}^{\leftrightarrow}$. If, in addition, $X$ is a finite-dimensional Banach space, then also $\mathcal{R}_{M}^{\leftrightarrow}=\mathcal{A}_{M}^{\leftrightarrow}$ is fulfilled.

(iv) $M$ is a $(p, T)$-attractor of $(\theta, \varphi)$ if and only if $M$ is a $\left(\theta_{T} p, T\right)$-repeller of $(\theta, \varphi)^{-1}$. We have $\mathfrak{A}_{M}^{(p, T)}=\mathfrak{R}_{M}^{\left(\theta_{T} p, T\right)}$.

Proof. (i) Let $M$ be a past attractor of $(\theta, \varphi)$, i.e., due to Proposition 2.1.2 (ii), there exists an $\eta>0$ such that for all $p \in P^{*}(M)$, there exists a $\hat{p} \in[p] \cap P^{*}(M)$ with

$$
\lim _{t \rightarrow \infty} d\left(\varphi\left(t, \theta_{-\tau-t} \hat{p}\right) U_{\eta}\left(M\left(\theta_{-\tau-t} \hat{p}\right)\right) \mid M\left(\theta_{-\tau} \hat{p}\right)\right)=0 \quad \text { for all } \tau \geq 0
$$

This is equivalent to

$$
\lim _{t \rightarrow \infty} d\left(\varphi^{-1}\left(-t, \theta^{-1}(\tau+t, \hat{p})\right) U_{\eta}\left(M\left(\theta^{-1}(\tau+t, \hat{p})\right)\right) \mid M\left(\theta^{-1}(\tau, \hat{p})\right)\right)=0 \quad \text { for all } \tau \geq 0,
$$

and due to Proposition 2.1.5 (v), this means that $M$ is a future repeller of $(\theta, \varphi)^{-1}$. In case $X$ is a 
finite-dimensional Banach space, the relation $\mathcal{A}_{M}^{\leftarrow}=\mathcal{R}_{M}$ follows from

$$
\begin{aligned}
\mathcal{A}_{M}^{\leftarrow}(p)=\{x \in X: & \text { There exists a neighborhood } U \text { of } x \text { such that } \\
& \left.\lim _{t \rightarrow \infty} d\left(\varphi\left(t, \theta_{-t} p\right)\left(M\left(\theta_{-t} p\right)+U\right) \mid M(p)\right)=0\right\} \\
=\{x \in X: & \text { There exists a neighborhood } U \text { of } x \text { such that } \\
& \left.\lim _{t \rightarrow \infty} d\left(\varphi^{-1}\left(-t, \theta^{-1}(t, p)\right)\left(M\left(\theta^{-1}(t, p)\right)+U\right) \mid M(p)\right)=0\right\}=\mathcal{R}_{M}(p)
\end{aligned}
$$

for all $p \in P^{*}(M)$. The relation $\mathfrak{A}_{M}^{\leftarrow}=\mathfrak{R}_{M}$ follows analogously.

The proofs of (ii), (iii) and (iv) are very similar to that of (i); we therefore omit them.

2.1.6 Existence and Uniqueness. In this subsection, criteria for the existence of attractors and repellers are formulated, and the question of their uniqueness is discussed.

First, the notions of past absorbing and future rejecting sets are introduced (these definitions are derived from Flandoli \& SCHMALFUSS [67] and KLOEDEN [93, 94]).

\subsubsection{Definition (PASt Absorbing and Future Rejecting Sets).}

(i) Let $B$ be a past nonautonomous set and $\mathcal{M}$ be a collection of past nonautonomous sets. Then, $B$ is called past absorbing with respect to $\mathcal{M}$ if for all $M \in \mathcal{M}$, we have $P^{*}(M) \subset P^{*}(B)$, and for all $M \in \mathcal{M}$ and $p \in P^{*}(M)$, there exists a $t^{*}>0$ such that

$$
\varphi\left(t, \theta_{-t} p\right) M\left(\theta_{-t} p\right) \subset B(p) \text { for all } t \geq t^{*} .
$$

(ii) We suppose that $(\theta, \varphi)$ is invertible. Let $B$ be a future nonautonomous set and $\mathcal{M}$ be a collection of future nonautonomous sets. Then, $B$ is called future rejecting with respect to $\mathcal{M}$ if for all $M \in \mathcal{M}$, we have $P^{*}(M) \subset P^{*}(B)$, and for all $M \in \mathcal{M}$ and $p \in P^{*}(M)$, there exists a $t^{*}>0$ such that

$$
\varphi\left(-t, \theta_{t} p\right) M\left(\theta_{t} p\right) \subset B(p) \text { for all } t \geq t^{*}
$$

2.1.24 REMARK. In case the past absorbing set is compact (which is a hypothesis of the next theorem), Definition 2.1.23 (i) is a nonautonomous generalization of the notion of the dissipative dynamical system (see, e.g., HALE [76]).

The following existence result is adapted from FLANDOLI \& SCHMALFUSS [67] (for related results in the context of random attractors, see also CRAUEL \& FLANDOLI [57, Theorem 3.11], SCHENK-Hoppé [158, Theorem 4.2] and SCHMALFUSS [159, 160]).

2.1.25 Theorem (Existence of $\mathcal{M}$-Past Attractors and $\mathcal{M}$-Future RePELLERS). The following statements are fulfilled:

(i) Let $\mathcal{M}$ be a collection of past nonautonomous sets and $B$ be a compact past absorbing set with respect to $\mathcal{M}$. Then, there exists an $\mathcal{M}$-past attractor $A$ fulfilling the representation 


$$
A(p)=\bigcap_{\tau \geq 0} \operatorname{cls} \bigcup_{t \geq \tau} \varphi\left(t, \theta_{-t} p\right) B\left(\theta_{-t} p\right) \quad \text { for all } p \in P^{*}(B) .
$$

If, in addition, $A \in \mathcal{M}$, then $A$ is uniquely determined. In case $B \in \mathcal{M}$, the relation $A \subset B$ is fulfilled.

(ii) We suppose that $(\theta, \varphi)$ is invertible. Let $\mathcal{M}$ be a collection of future nonautonomous sets and $B$ be a compact future rejecting set with respect to $\mathcal{M}$. Then, there exists an $\mathcal{M}$-future repeller $R$ fulfilling the representation

$$
R(p)=\bigcap_{\tau \geq 0} \operatorname{cls} \bigcup_{t \geq \tau} \varphi\left(-t, \theta_{t} p\right) B\left(\theta_{t} p\right) \quad \text { for all } p \in P^{*}(B) .
$$

If, in addition, $R \in \mathcal{M}$, then $R$ is uniquely determined. In case $B \in \mathcal{M}$, the relation $R \subset B$ is fulfilled.

Proof. For assertion (i), see Flandoli \& Schmalfuss [67, Theorem 3.5], (ii) follows from (i) using Proposition 2.1.22.

In case the collection $\mathcal{M}$ of the above theorem contains a neighborhood of the set $B$, existence results for past attractors and future repellers follow directly.

\subsubsection{Corollary (Existence of Past Attractors And Future Repellers).}

The following statements are fulfilled:

(i) Let $\mathcal{M}$ be a collection of past nonautonomous sets, $B$ be a compact past absorbing set with respect to $\mathcal{M}$ and $\eta>0$ such that the past nonautonomous set $\bar{B}$, defined by

$$
\bar{B}(p):=U_{\eta}(B(p)) \text { for all } p \in P^{*}(B)
$$

lies in $\mathcal{M}$. Then, the $\mathcal{M}$-past attractor of Theorem 2.1.25 (i) is also a past attractor.

(ii) We suppose that $(\theta, \varphi)$ is invertible. Let $\mathcal{M}$ be a collection of future nonautonomous sets, $B$ be a compact future rejecting set with respect to $\mathcal{M}$ and $\eta>0$ such that the future nonautonomous set $\bar{B}$, defined by

$$
\bar{B}(p):=U_{\eta}(B(p)) \text { for all } p \in P^{*}(B)
$$

lies in $\mathcal{M}$. Then, the $\mathcal{M}$-future repeller of Theorem 2.1.25 (ii) is also a future repeller.

ProOF. The assertions follow directly from Definition 2.1.1 (ii) and Definition 2.1.4 (iv).

In the following proposition, the question of local uniqueness and nonuniqueness for nonautonomous attractors and repellers is discussed.

2.1.27 Proposition (Local UniQueness and Nonuniqueness). The following statements are fulfilled:

(i) Let $A_{1}$ and $A_{2}$ be past attractors such that $A_{1}(p) \neq A_{2}(p)$ for all $p \in P$. Then, we have 


$$
\liminf _{t \rightarrow \infty} d_{H}\left(\left(A_{1}\left(\theta_{-t} p\right), A_{2}\left(\theta_{-t} p\right)\right) \geq \min \left\{\mathfrak{A}_{A_{1}}^{\leftarrow}, \mathfrak{A}_{A_{2}}^{\leftarrow}\right\} \quad \text { for all } p \in P\right.
$$

(ii) We suppose that $(\theta, \varphi)$ is invertible. Let $R_{1}$ and $R_{2}$ be future repellers such that $R_{1}(p) \neq R_{2}(p)$ for all $p \in P$. Then, we have

$$
\liminf _{t \rightarrow \infty} d_{H}\left(R_{1}\left(\theta_{t} p\right), R_{2}\left(\theta_{t} p\right)\right) \geq \min \left\{\mathfrak{R}_{R_{1}}, \mathfrak{R}_{R_{2}}\right\} \quad \text { for all } p \in P
$$

(iii) We suppose that $(\theta, \varphi)$ is invertible. Let $R_{1}$ be a past repeller. Then, for all $p \in P^{*}\left(R_{1}\right)$ and $\delta \in\left(0, \mathfrak{R}_{R_{1}}^{\leftarrow}\right)$, there exists a $\hat{p} \in[p] \cap P^{*}\left(R_{1}\right)$ such that for all $\tau \geq 0$ and compact sets $R_{2}\left(\theta_{-\tau} \hat{p}\right) \subset X$ with

$$
R_{1}\left(\theta_{-\tau} \hat{p}\right) \subset R_{2}\left(\theta_{-\tau} \hat{p}\right) \subset \operatorname{cls} U_{\delta}\left(R_{1}\left(\theta_{-\tau} \hat{p}\right)\right),
$$

the past nonautonomous set $R_{2}$, defined by

$$
R_{2}(\bar{p}):=\left\{\begin{array}{cll}
\varphi\left(-t, \theta_{-\tau} \hat{p}\right) R_{2}\left(\theta_{-\tau} \hat{p}\right) & : \bar{p}=\theta_{-\tau-t} \hat{p} \quad \text { for some } t \in \mathbb{T}_{0}^{+} \\
R_{1}(\bar{p}) & : \bar{p} \in P^{*}\left(R_{1}\right) \backslash[p]
\end{array},\right.
$$

is also a past repeller with $\mathfrak{R}_{R_{1}}^{\leftarrow}=\mathfrak{R}_{R_{2}}^{\leftarrow}$

(iv) Let $A_{1}$ be a future attractor. Then, for all $p \in P^{*}\left(A_{1}\right)$ and $\delta \in\left(0, \mathfrak{A}_{A_{1}}\right)$, there exists a $\hat{p} \in[p] \cap P^{*}\left(A_{1}\right)$ such that for all $\tau \geq 0$ and compact sets $A_{2}\left(\theta_{\tau} \hat{p}\right) \subset X$ with

$$
A_{1}\left(\theta_{\tau} \hat{p}\right) \subset A_{2}\left(\theta_{\tau} \hat{p}\right) \subset \operatorname{cls} U_{\delta}\left(A_{1}\left(\theta_{\tau} \hat{p}\right)\right)
$$

the future nonautonomous set $A_{2}$, defined by

$$
A_{2}(\bar{p}):=\left\{\begin{array}{cll}
\varphi\left(t, \theta_{\tau} \hat{p}\right) A_{2}\left(\theta_{\tau} \hat{p}\right) & : \bar{p}=\theta_{\tau+t} \hat{p} \quad \text { for some } t \in \mathbb{T}_{0}^{+} \\
A_{1}(\bar{p}) & : \bar{p} \in P^{*}\left(A_{1}\right) \backslash[p]
\end{array},\right.
$$

is also a future attractor with $\mathfrak{A}_{A_{1}}=\mathfrak{A A}_{A_{2}}$.

\subsubsection{REMARKS.}

(i) The form of (local) nonuniqueness of past repellers and future attractors is weak in the sense that, for instance, the past repellers $R_{1}$ and $R_{2}$ from (iii) fulfill

$$
\lim _{t \rightarrow \infty} d_{H}\left(R_{1}\left(\theta_{-t} p\right), R_{2}\left(\theta_{-t} p\right)\right)=0 \quad \text { for all } p \in P .
$$

(ii) Since all-time attractors (repellers, respectively) are past attractors (future repellers, respectively) (cf. remark after Definition 2.1.7), they also fulfill a uniqueness result similar to (i) ((ii), respectively).

(iii) Concerning finite-time attractors and repellers, it is not possible to show uniqueness and nonuniqueness results. More precisely, it is possible that in every neighborhood of a $(p, T)$ attractor lies another $(p, T)$-attractor and an invariant $(p, T)$-nonautonomous set which is not a $(p, T)$-attractor.

(iv) In case $X$ is a finite-dimensional Banach space, in (iii) ((iv), respectively), not only the relation $\mathfrak{R}_{R_{1}}^{\leftarrow}=\mathfrak{R}_{R_{2}}^{\leftarrow}\left(\mathfrak{A}_{A_{1}}^{\leftarrow}=\mathfrak{A A}_{A_{2}}^{\leftarrow}\right.$, respectively) but also $\mathcal{R}_{R_{1}}^{\leftarrow}=\mathcal{R}_{R_{2}}^{\leftarrow}\left(\mathcal{A}_{A_{1}}^{\leftarrow}=\mathcal{A}_{A_{2}}^{\leftarrow}\right.$, respectively) holds. 
ProOF OF PROPOSITION 2.1.27. We will only prove the statements (i) and (iii), since the proofs of (ii) and (iv) are similar.

(i) Assume to the contrary that there exists a $p \in P$ with

$$
\liminf _{t \rightarrow \infty} d_{H}\left(A_{1}\left(\theta_{-t} p\right), A_{2}\left(\theta_{-t} p\right)\right)<\min \left\{\mathfrak{A}_{A_{1}}^{\leftarrow}, \mathfrak{A}_{A_{2}}^{\leftarrow}\right\}
$$

Hence, there exist a $\beta<\min \left\{\mathfrak{A}_{A_{1}}^{\leftarrow}, \mathfrak{A}_{A_{2}}^{\leftarrow}\right\}$ and a sequence $\left\{t_{n}\right\}_{n \in \mathbb{N}}$ with $\lim _{n \rightarrow \infty} t_{n}=\infty$ and

$$
A_{2}\left(\theta_{-t_{n}} p\right) \subset U_{\beta}\left(A_{1}\left(\theta_{-t_{n}} p\right)\right) \text { for all } n \in \mathbb{N} \text {. }
$$

Due to Definition 2.1.15, this implies the existence of a $\tau \geq 0$ such that

$$
\lim _{n \rightarrow \infty} d(\underbrace{\varphi\left(t_{n}-\tau, \theta_{-t_{n}} p\right) A_{2}\left(\theta_{-t_{n}} p\right)}_{=A_{2}\left(\theta_{-\tau} p\right)} \mid A_{1}\left(\theta_{-\tau} p\right))=0 .
$$

This means that $d\left(A_{2}\left(\theta_{-\tau} p\right) \mid A_{1}\left(\theta_{-\tau} p\right)\right)=0$, and hence, $d\left(A_{2}(p) \mid A_{1}(p)\right)=0$. Analogously, one can show $d\left(A_{1}(p) \mid A_{2}(p)\right)=0$. This implies $A_{1}(p)=A_{2}(p)$, since $A_{1}(p)$ and $A_{2}(p)$ are compact, and this contradiction finishes the proof of (i).

(iii) We choose $p \in P^{*}\left(R_{1}\right)$ and $\delta, \eta \in\left(0, \mathfrak{R}_{R_{1}}^{\leftarrow}\right)$ arbitrarily and define

$$
\beta:=\frac{1}{2}\left(\max \{\delta, \eta\}+\mathfrak{R}_{R_{1}}^{\leftarrow}\right) \in\left(\max \{\delta, \eta\}, \mathfrak{R}_{R_{1}}^{\leftarrow}\right)
$$

Due to Definition 2.1.15, there exists a $\hat{p} \in[p] \cap P^{*}\left(R_{1}\right)$ such that

$$
\lim _{t \rightarrow \infty} d\left(\varphi\left(-t, \theta_{-\tau} \hat{p}\right) U_{\beta}\left(R_{1}\left(\theta_{-\tau} \hat{p}\right)\right) \mid R_{1}\left(\theta_{-\tau-t} \hat{p}\right)\right)=0 \quad \text { for all } \tau \geq 0 .
$$

We choose $\tau \geq 0$ and a compact set $R_{2}\left(\theta_{-\tau} \hat{p}\right) \subset X$ with

$$
R_{1}\left(\theta_{-\tau} \hat{p}\right) \subset R_{2}\left(\theta_{-\tau} \hat{p}\right) \subset \operatorname{cls} U_{\delta}\left(R_{1}\left(\theta_{-\tau} \hat{p}\right)\right)
$$

and define the nonautonomous set $R_{2}$ as stated in the proposition. Because of (2.3), there exists a $t^{*} \geq 0$ such that

$$
d(\underbrace{\varphi\left(-t, \theta_{-\tau} \hat{p}\right) R_{2}\left(\theta_{-\tau} \hat{p}\right)}_{=R_{2}\left(\theta_{-\tau-t} \hat{p}\right)} \mid R_{1}\left(\theta_{-\tau-t} \hat{p}\right))<\frac{\mathfrak{R}_{R_{1}}^{\leftarrow}-\beta}{2} \quad \text { for all } t \geq t^{*}
$$

Since $\frac{1}{2}\left(\mathfrak{R}_{R_{1}}^{\leftarrow}-\beta\right)+\eta<\beta$, this implies using (2.3) the relation

$$
\lim _{t \rightarrow \infty} d\left(\varphi\left(-t, \theta_{-\tau-s} \hat{p}\right) U_{\eta}\left(R_{2}\left(\theta_{-\tau-s} \hat{p}\right)\right) \mid R_{1}\left(\theta_{-\tau-s-t} \hat{p}\right)\right)=0 \quad \text { for all } s \geq t^{*} .
$$

Because of $R_{1} \subset R_{2}$, we obtain

$$
\lim _{t \rightarrow \infty} d\left(\varphi\left(-t, \theta_{-\tau-s} \hat{p}\right) U_{\eta}\left(R_{2}\left(\theta_{-\tau-s} \hat{p}\right)\right) \mid R_{2}\left(\theta_{-\tau-s-t} \hat{p}\right)\right)=0 \quad \text { for all } s \geq t^{*}
$$

and this means that $R_{2}$ is a past repeller with $\mathfrak{R}_{R_{2}}^{\leftarrow} \geq \eta$ (please note that $R_{1}(\bar{p})=R_{2}(\bar{p})$ for all $\left.\bar{p} \in P^{*}\left(R_{1}\right) \backslash[p]\right)$. Hence, $\mathfrak{R}_{R_{2}}^{\leftarrow} \geq \mathfrak{R}_{R_{1}}^{\leftarrow}\left(\eta\right.$ has been chosen arbitrarily). The relation $\mathfrak{R}_{R_{2}}^{\leftarrow} \leq \mathfrak{R}_{R_{1}}^{\leftarrow}$ can be obtained from

$$
\lim _{t \rightarrow \infty} d\left(R_{2}\left(\theta_{-t} p\right) \mid R_{1}\left(\theta_{-t} p\right)\right)=0 .
$$


This finishes the proof of this proposition.

In the following lemma, attraction (repulsion, respectively) areas of attractive (repulsive, respectively) solutions lying in repellers (attractors, respectively) are analyzed.

2.1.29 LemmA. We suppose that $X$ is a finite-dimensional Banach space and $(\theta, \varphi)$ is invertible. Then, the following statements are fulfilled:

(i) Let $R$ be a past repeller and $\mu: \mathcal{O}^{-}(p) \rightarrow X$ be a past attractive solution with

$$
\mu\left(\theta_{-t} p\right) \in \operatorname{int} R\left(\theta_{-t} p\right) \quad \text { for all } t \geq 0 \text {. }
$$

Then, we have

$$
\liminf _{t \rightarrow \infty}\left(R\left(\theta_{-t} p\right)-\mu\left(\theta_{-t} p\right)\right) \supset \mathcal{A}_{\mu}^{\leftarrow}
$$

(ii) Let $A$ be a past attractor and $\mu: \mathcal{O}^{-}(p) \rightarrow X$ be a past repulsive solution with

$$
\mu\left(\theta_{-t} p\right) \in A\left(\theta_{-t} p\right) \quad \text { for all } t \geq 0 \text {. }
$$

Then, the relation $A\left(\theta_{-t} p\right)-\mu\left(\theta_{-t} p\right) \supset \mathcal{R}_{\mu}^{\leftarrow}\left(\theta_{-t} p\right)$ holds for all $t \geq 0$, and we thus have

$$
\liminf _{t \rightarrow \infty}\left(A\left(\theta_{-t} p\right)-\mu\left(\theta_{-t} p\right)\right) \supset \mathcal{R}_{\mu}^{\leftarrow}
$$

(iii) Let $A$ be a future attractor and $\mu: \mathcal{O}^{+}(p) \rightarrow X$ be a future repulsive solution with

$$
\mu\left(\theta_{t} p\right) \in \operatorname{int} A\left(\theta_{t} p\right) \quad \text { for all } t \geq 0 \text {. }
$$

Then, we have

$$
\liminf _{t \rightarrow \infty}\left(A\left(\theta_{t} p\right)-\mu\left(\theta_{t} p\right)\right) \supset \mathcal{R}_{\mu}
$$

(iv) Let $R$ be a future repeller and $\mu: \mathcal{O}^{+}(p) \rightarrow X$ be a future attractive solution with

$$
\mu\left(\theta_{t} p\right) \in R\left(\theta_{t} p\right) \quad \text { for all } t \geq 0 \text {. }
$$

Then, the relation $R\left(\theta_{t} p\right)-\mu\left(\theta_{t} p\right) \supset \mathcal{A}_{\mu}\left(\theta_{t} p\right)$ holds for all $t \geq 0$, and we thus have

$$
\liminf _{t \rightarrow \infty}\left(R\left(\theta_{t} p\right)-\mu\left(\theta_{t} p\right)\right) \supset \mathcal{A}_{\mu}
$$

\subsubsection{REMARKS.}

(i) This lemma implies that (past and future) attractors or repellers containing repulsive or attractive solutions, respectively, are nontrivial, i.e., their fibers are no singletons.

(ii) Since the notions of all-time attractivity and repulsivity are stronger than those of past and future attractivity and repulsivity (cf. remark after Definition 2.1.7), the assertions of the above lemma are also applicable for all-time attractors and repellers.

Proof OF Lemma 2.1.29. Because of Proposition 2.1.22, it is sufficient to show the statements (i) and (ii).

(i) We choose $x \in \mathcal{A}_{\mu}^{\leftarrow}$. Due to the hypotheses, there exists an $\eta>0$ such that

$$
\varphi(-t, p) U_{\eta}(\mu(p)) \subset R\left(\theta_{-t} p\right) \quad \text { for all } t \geq 0 \text {. }
$$


Since $x \in \mathcal{A}_{\mu}^{\leftarrow}$, there exists a $\tau>0$ with

$$
\varphi\left(t, \theta_{-t} p\right)\left(\mu\left(\theta_{-t} p\right)+x\right) \in U_{\eta}(\mu(p)) \text { for all } t \geq \tau .
$$

Hence, we have

$$
\mu\left(\theta_{-t} p\right)+x=\varphi(-t, p) \varphi\left(t, \theta_{-t} p\right)\left(\mu\left(\theta_{-t} p\right)+x\right) \in R\left(\theta_{-t} p\right) \text { for all } t \geq \tau .
$$

Therefore, $x \in \liminf _{t \rightarrow \infty}\left(R\left(\theta_{-t} p\right)-\mu\left(\theta_{-t} p\right)\right)$.

(ii) We choose $x \in \mathcal{R}_{\mu}^{\leftarrow}(p)$ and $\delta>0$ arbitrarily. Since $\mu$ lies in $A$, there exist $\eta>0$ and $\tau>0$ such that

$$
\varphi\left(t, \theta_{-t} p\right) U_{\eta}\left(\mu\left(\theta_{-t} p\right)\right) \subset U_{\delta}(A(p)) \text { for all } t \geq \tau .
$$

Since $x \in \mathcal{R}_{\mu}^{\leftarrow}(p)$, there exists a $\hat{\tau}>0$ with

$$
\varphi(-t, p)(\mu(p)+x) \in U_{\eta}\left(\mu\left(\theta_{-t} p\right)\right) \text { for all } t \geq \hat{\tau} .
$$

Hence, with $t:=\max \{\tau, \hat{\tau}\}$, the relation

$$
\mu(p)+x=\varphi\left(t, \theta_{-t} p\right) \varphi(-t, p)(\mu(p)+x) \in U_{\delta}(A(p))
$$

holds. Since $\delta$ has been chosen arbitrarily and $A(p)$ is compact, we have $\mu(p)+x \in A(p)$, and therefore, $\mu(p)+\mathcal{R}_{\mu}^{\leftarrow}(p) \subset A(p)$ is fulfilled. The assertion follows directly from Proposition 2.1.19 (i).

In the following theorem, sufficient conditions are derived to guarantee the existence of a nonautonomous attractor (repeller, respectively) which contains a nonautonomous repulsive (attractive, respectively) solution.

2.1.31 TheOrem (EXISTENCE OF NONAUtOnOMOUS ATTRACTORS AND REPELLERS). Assume, $X$ is a finite-dimensional Banach space and $(\theta, \varphi)$ is invertible. Then, the following statements are fulfilled:

(i) We suppose that $\mu: \mathcal{O}^{-}(p) \rightarrow X$ is a past attractive solution such that $\mathcal{A}_{\mu}^{\leftarrow}$ is bounded and there exist $\varepsilon>0$ and $s>0$ with

$$
\lim _{t \rightarrow \infty} d\left(\varphi\left(-t, \theta_{-\tau} p\right) U_{\varepsilon}\left(\mu\left(\theta_{-\tau} p\right)+\mathcal{A}_{\mu}^{\leftarrow}\right) \mid \mu\left(\theta_{-\tau-t} p\right)+\mathcal{A}_{\mu}^{\leftarrow}\right)=0 \quad \text { for all } \tau \geq s
$$

Then, there exists a $\beta>0$ such that the invariant and compact past nonautonomous set $R$, defined by

$$
R\left(\theta_{-t-s} p\right):=\varphi\left(-t, \theta_{-s} p\right) \operatorname{cls} U_{\beta}\left(\mu\left(\theta_{-s} p\right)\right) \quad \text { for all } t \geq 0,
$$

is a past repeller fulfilling

$$
\mathcal{A}_{\mu}^{\leftarrow} \subset \liminf _{t \rightarrow \infty}\left(R\left(\theta_{-t} p\right)-\mu\left(\theta_{-t} p\right)\right) \subset \limsup _{t \rightarrow \infty}\left(R\left(\theta_{-t} p\right)-\mu\left(\theta_{-t} p\right)\right) \subset \operatorname{cls} \mathcal{A}_{\mu}^{\leftarrow}
$$

(ii) We suppose that $\mu: \mathcal{O}^{-}(p) \rightarrow X$ is a past repulsive solution such that $\mathcal{R}_{\mu}^{\leftarrow}$ is bounded and there exists an $\eta>0$ such that for all $\varepsilon>0$, there exists an $s>0$ such that for all $\tau \geq s$, there is a $T>0$ with

$$
\varphi\left(t, \theta_{-\tau-t} p\right) U_{\eta}\left(\mu\left(\theta_{-\tau-t} p\right)+\mathcal{R}_{\mu}^{\leftarrow}\right) \subset U_{\varepsilon}\left(\mu\left(\theta_{-\tau} p\right)+\mathcal{R}_{\mu}^{\leftarrow}\right) \quad \text { for all } t \geq T
$$

Then, there exists a past attractor $A \subset \mathcal{O}^{-}(p) \times X$ fulfilling 


$$
\mathcal{R}_{\mu}^{\leftarrow} \subset \liminf _{t \rightarrow \infty}\left(A\left(\theta_{-t} p\right)-\mu\left(\theta_{-t} p\right)\right) \subset \limsup _{t \rightarrow \infty}\left(A\left(\theta_{-t} p\right)-\mu\left(\theta_{-t} p\right)\right) \subset \operatorname{cls} \mathcal{R}_{\mu}^{\leftarrow}
$$

(iii) We suppose that $\mu: \mathcal{O}^{+}(p) \rightarrow X$ is a future repulsive solution such that $\mathcal{R}_{\mu}$ is bounded and there exist $\varepsilon>0$ and $s>0$ with

$$
\lim _{t \rightarrow \infty} d\left(\varphi\left(t, \theta_{\tau} p\right) U_{\varepsilon}\left(\mu\left(\theta_{\tau} p\right)+\mathcal{R}_{\mu}\right) \mid \mu\left(\theta_{t+\tau} p\right)+\mathcal{R}_{\mu}\right)=0 \quad \text { for all } \tau \geq s .
$$

Then, there exists a $\beta>0$ such that the invariant and compact future nonautonomous set $A$, defined by

$$
A\left(\theta_{t+s} p\right):=\varphi\left(t, \theta_{s} p\right) \operatorname{cls} U_{\beta}\left(\mu\left(\theta_{s} p\right)\right) \quad \text { for all } t \geq 0,
$$

is a future attractor fulfilling

$$
\mathcal{R}_{\mu} \overrightarrow{\liminf _{t \rightarrow \infty}}\left(A\left(\theta_{t} p\right)-\mu\left(\theta_{t} p\right)\right) \subset \limsup _{t \rightarrow \infty}\left(A\left(\theta_{t} p\right)-\mu\left(\theta_{t} p\right)\right) \subset \operatorname{cls} \mathcal{R}_{\mu} .
$$

(iv) We suppose that $\mu: \mathcal{O}^{+}(p) \rightarrow X$ is a future attractive solution such that $\mathcal{A}_{\mu}$ is bounded and there exists an $\eta>0$ such that for all $\varepsilon>0$, there exists an $s>0$ such that for all $\tau \geq s$, there is a $T>0$ with

$$
\varphi\left(-t, \theta_{\tau+t} p\right) U_{\eta}\left(\mu\left(\theta_{\tau+t} p\right)+\mathcal{A}_{\mu}\right) \subset U_{\varepsilon}\left(\mu\left(\theta_{\tau} p\right)+\mathcal{A}_{\mu}\right) \quad \text { for all } t \geq T
$$

Then, there exists a future repeller $R \subset \mathcal{O}^{+}(p) \times X$ fulfilling

$$
\mathcal{A}_{\mu} \overrightarrow{\liminf _{t \rightarrow \infty}}\left(R\left(\theta_{t} p\right)-\mu\left(\theta_{t} p\right)\right) \subset \limsup _{t \rightarrow \infty}\left(R\left(\theta_{t} p\right)-\mu\left(\theta_{t} p\right)\right) \subset \operatorname{cls} \mathcal{A}_{\mu}
$$

Proof. Due to Proposition 2.1.22, it is sufficient to show the statements (i) and (ii).

(i) We choose a $\beta>0$ with cls $U_{\beta}(0) \subset U_{\varepsilon}\left(\mathcal{A}_{\mu}^{\leftarrow}\right)$ and define

$$
R\left(\theta_{-s-t} p\right):=\varphi\left(-t, \theta_{-s} p\right) \operatorname{cls} U_{\beta}\left(\mu\left(\theta_{-s} p\right)\right) \text { for all } t \geq 0 .
$$

This means that

$$
R\left(\theta_{-s-t} p\right) \subset \varphi\left(-t, \theta_{-s} p\right) U_{\varepsilon}\left(\mu\left(\theta_{-s} p\right)+\mathcal{A}_{\mu}^{\leftarrow}\right) \quad \text { for all } t \geq 0
$$

Moreover,

$$
\begin{aligned}
& \lim _{t \rightarrow \infty} d\left(R\left(\theta_{-t} p\right) \mid \mu\left(\theta_{-t} p\right)+\mathcal{A}_{\mu}^{\leftarrow}\right) \\
\stackrel{(2.5)}{\leq} & \lim _{t \rightarrow \infty} d\left(\varphi\left(-t, \theta_{-s} p\right) U_{\varepsilon}\left(\mu\left(\theta_{-s} p\right)+\mathcal{A}_{\mu}^{\leftarrow}\right) \mid \mu\left(\theta_{-s-t} p\right)+\mathcal{A}_{\mu}^{\leftarrow}\right) \stackrel{(2.4)}{=} 0
\end{aligned}
$$

is fulfilled, and therefore,

$$
\limsup _{t \rightarrow \infty}\left(R\left(\theta_{-t} p\right)-\mu\left(\theta_{-t} p\right)\right) \subset \operatorname{cls} \mathcal{A}_{\mu}^{\leftarrow}
$$

holds. Next, we show that $R$ is a past repeller. Suppose that

$$
\delta:=\liminf _{t \rightarrow \infty} d\left(\mu\left(\theta_{-t} p\right)+\mathcal{A}_{\mu}^{\leftarrow} \mid R\left(\theta_{-t} p\right)\right)>0
$$


is fulfilled. Since cls $\mathcal{A}_{\mu}^{\leftarrow}$ is compact, there exist an $n \in \mathbb{N}$ and elements $x_{1}, \ldots, x_{n} \in \operatorname{cls} \mathcal{A}_{\mu}^{\leftarrow}$ such that

$$
\operatorname{cls} \mathcal{A}_{\mu}^{\leftarrow} \subset \bigcup_{i=1}^{n} U_{\frac{\delta}{4}}\left(x_{i}\right) .
$$

For all $i \in\{1, \ldots, n\}$, we choose arbitrary elements

$$
y_{i} \in U_{\frac{\delta}{4}}\left(x_{i}\right) \cap \mathcal{A}_{\mu}^{\leftarrow}
$$

Then, the set $C:=\left\{y_{1}, \ldots, y_{n}\right\}$ is a compact subset of $\mathcal{A}_{\mu}^{\leftarrow}$ which fulfills

$$
d\left(\mathcal{A}_{\mu}^{\leftarrow} \mid C\right) \leq \frac{\delta}{2}
$$

It follows from Proposition 2.1.16 (i) that there exists a $\tilde{t} \geq 0$ such that

$$
\varphi\left(t, \theta_{-s-t} p\right)\left(\mu\left(\theta_{-s-t} p\right)+C\right) \subset U_{\beta}\left(\mu\left(\theta_{-s} p\right)\right) \subset R\left(\theta_{-s} p\right) \text { for all } t \geq \tilde{t} .
$$

Hence, due to the invariance of $R$, we obtain

$$
\lim _{t \rightarrow \infty} d\left(\mu\left(\theta_{-t} p\right)+C \mid R\left(\theta_{-t} p\right)\right)=0 .
$$

Using Lemma A.2.2, this implies

$$
\begin{aligned}
& \liminf _{t \rightarrow \infty} d\left(\mu\left(\theta_{-t} p\right)+\mathcal{A}_{\mu}^{\leftarrow} \mid R\left(\theta_{-t} p\right)\right) \\
\leq & \liminf _{t \rightarrow \infty}\left(d\left(\mu\left(\theta_{-t} p\right)+\mathcal{A}_{\mu}^{\leftarrow} \mid \mu\left(\theta_{-t} p\right)+C\right)+d\left(\mu\left(\theta_{-t} p\right)+C \mid R\left(\theta_{-t} p\right)\right)\right) \stackrel{(2.7)}{\leq} \frac{\delta}{2}
\end{aligned}
$$

This is a contradiction to (2.6). Therefore,

$$
\lim _{t \rightarrow \infty} d\left(\mu\left(\theta_{-t} p\right)+\mathcal{A}_{\mu}^{\leftarrow} \mid R\left(\theta_{-t} p\right)\right)=0
$$

is fulfilled. Furthermore, there exists a $\hat{t} \geq 0$ with

$$
\begin{aligned}
& d\left(R\left(\theta_{-s-\tau} p\right) \mid \mu\left(\theta_{-s-\tau} p\right)+\mathcal{A}_{\mu}^{\leftarrow}\right) \\
& \stackrel{(2.5)}{\leq} d\left(\varphi\left(-\tau, \theta_{-s} p\right) U_{\varepsilon}\left(\mu\left(\theta_{-s} p\right)+\mathcal{A}_{\mu}^{\leftarrow}\right) \mid \mu\left(\theta_{-s-\tau} p\right)+\mathcal{A}_{\mu}^{\leftarrow}\right) \stackrel{(2.4)}{<} \frac{\varepsilon}{2} \quad \text { for all } \tau \geq \hat{t}
\end{aligned}
$$

Hence, we have

$$
\begin{aligned}
U_{\varepsilon}\left(\mu\left(\theta_{-s-\tau} p\right)+\mathcal{A}_{\mu}^{\leftarrow}\right) & \supset U_{\frac{\varepsilon}{2}}\left(U_{\frac{\varepsilon}{2}}\left(\mu\left(\theta_{-s-\tau} p\right)+\mathcal{A}_{\mu}^{\leftarrow}\right)\right) & \\
& \supset U_{\frac{\varepsilon}{2}}\left(R\left(\theta_{-s-\tau} p\right)\right) & \text { for all } \tau \geq \hat{t}
\end{aligned}
$$

For all $\tau \geq \hat{t}$, the inequality

$$
\begin{array}{cl}
\stackrel{\lim _{t \rightarrow \infty} d\left(\varphi\left(-t, \theta_{-s-\tau} p\right) U_{\frac{\varepsilon}{2}}\left(R\left(\theta_{-s-\tau} p\right)\right) \mid R\left(\theta_{-s-\tau-t} p\right)\right)}{\leq} \lim _{t \rightarrow \infty} d\left(\varphi\left(-t, \theta_{-s-\tau} p\right) U_{\varepsilon}\left(\mu\left(\theta_{-s-\tau} p\right)+\mathcal{A}_{\mu}^{\leftarrow}\right) \mid R\left(\theta_{-s-\tau-t} p\right)\right) \\
\stackrel{\text { Lemma A.2.2 }}{\leq} \lim _{t \rightarrow \infty} d\left(\varphi\left(-t, \theta_{-s-\tau} p\right) U_{\varepsilon}\left(\mu\left(\theta_{-s-\tau} p\right)+\mathcal{A}_{\mu}^{\leftarrow}\right) \mid \mu\left(\theta_{-s-\tau-t} p\right)+\mathcal{A}_{\mu}^{\leftarrow}\right)+ \\
\\
\lim _{t \rightarrow \infty} d\left(\mu\left(\theta_{-s-\tau-t} p\right)+\mathcal{A}_{\mu}^{\leftarrow} \mid R\left(\theta_{-s-\tau-t} p\right)\right) \\
\stackrel{(2.4),(2.8)}{=} \quad 0
\end{array}
$$


holds, and this means that $R$ is a past repeller. The relation

$$
\liminf _{t \rightarrow \infty}\left(R\left(\theta_{-t} p\right)-\mu\left(\theta_{-t} p\right)\right) \supset \mathcal{A}_{\mu}^{\leftarrow}
$$

follows from Lemma 2.1.29 (i).

(ii) We define the past nonautonomous set $M$ by its fibers

$$
M\left(\theta_{-t} p\right):=U_{\eta}\left(\mu(t)+\mathcal{R}_{\mu}^{\leftarrow}\right) \quad \text { for all } t \geq 0
$$

Due to the hypotheses, the fibers of a compact past absorbing set $B$ with respect to $\{M\}$ can be defined with the following property: For all $\varepsilon>0$, there exists a $\tau \geq 0$ such that

$$
\mathcal{R}_{\mu}^{\leftarrow}+\mu\left(\theta_{-t} p\right) \subset B\left(\theta_{-t} p\right) \subset U_{\varepsilon}\left(\mathcal{R}_{\mu}^{\leftarrow}+\mu\left(\theta_{-t} p\right)\right) \text { for all } t \geq \tau
$$

Therefore, Theorem 2.1.25 implies the existence of an $\{M\}$-past attractor $A \subset B$ fulfilling

$$
\limsup _{t \rightarrow \infty}\left(A\left(\theta_{-t} p\right)-\mu\left(\theta_{-t} p\right)\right) \subset \limsup _{t \rightarrow \infty}\left(B\left(\theta_{-t} p\right)-\mu\left(\theta_{-t} p\right)\right) \stackrel{(2.10)}{\subset} \operatorname{cls} \mathcal{R}_{\mu}^{\leftarrow}
$$

Due to Corollary 2.1.26, $A$ is also a past attractor. The relation

$$
\liminf _{t \rightarrow \infty}\left(A\left(\theta_{-t} p\right)-\mu\left(\theta_{-t} p\right)\right) \supset \mathcal{R}_{\mu}^{\leftarrow}
$$

follows from Lemma 2.1.29 (ii).

\subsection{Other Notions of AtTRACTIVITY AND REPUlSIVITY}

In this section, other notions of attractivity and repulsivity from the literature are discussed with respect to their relationship to the definitions of the previous section. In the first subsection, the well-known theory of stability in the sense of Lyapunov is treated, and in Subsection 2.2.2, it is indicated that the notions of past (future, all-time, respectively) attractor and repeller are generalizations of the concept of attractor and repeller introduced in CONLEY [52]. Finally, the last subsection is devoted to the theory of nonautonomous attractors.

2.2.1 Theory of Stability in the Sense of Lyapunov. Several different forms of stability are examined in the literature. Most articles in this area, however, deal with the concept of stability in the sense of Lyapunov, which has been introduced by LYAPUNOV in his thesis [109] (see $[111,112]$ for translations into French and English). We shortly review the basic definitions of this theory in the context of nonautonomous differential equations (see also the classical books from CESARI [39] and HAHN [73]). An analogous theory exists for nonautonomous difference equations (see, e.g., AGARWAL [2]). Let

$$
\dot{x}=f(t, x)
$$

be a nonautonomous differential equation with a function $f: D \subset \mathbb{R} \times \mathbb{R}^{N} \rightarrow \mathbb{R}^{N}$ satisfying conditions guaranteeing local existence and uniqueness of solutions (see Appendix A.1). The general solution of (2.11) is denoted by $\lambda$. A solution $\mu:(\tau, \infty) \rightarrow \mathbb{R}^{N}$ is called Lyapunov-stable if for all $t_{0}>\tau$ and $\varepsilon>0$, there exists a $\delta=\delta\left(t_{0}, \varepsilon\right)>0$ with

$$
\lambda\left(t, t_{0}, U_{\delta}\left(\mu\left(t_{0}\right)\right)\right) \subset U_{\varepsilon}(\mu(t)) \text { for all } t \geq t_{0} .
$$


Furthermore, a solution $\mu:(\tau, \infty) \rightarrow \mathbb{R}^{N}$ is called Lyapunov-attractive if for all $t_{0}>\tau$, there exists an $\eta=\eta\left(t_{0}\right)>0$ such that

$$
\lim _{t \rightarrow \infty}\left\|\lambda\left(t, t_{0}, x\right)-\mu(t)\right\|=0 \quad \text { for all } x \in U_{\eta}\left(\mu\left(t_{0}\right)\right) .
$$

There exist counterexamples in dimensions greater than one which show that not every Lyapunovattractive solution is Lyapunov-stable (see, e.g., AULBACH [14, Beispiel 7.4.16, p. 325] and BHATIA \& SZEGÖ [30, p. 59]). However, if a solution is both Lyapunov-stable and Lyapunovattractive, we call this solution Lyapunov-asymptotically stable. If in the definition of the Lyapunov-stable solution, $\delta$ is independent of $t_{0}$, we call this solution uniformly Lyapunov-stable. In case $\eta$ is independent of $t_{0}$ in the definition of the Lyapunov-attractive solution, we call this solution uniformly Lyapunov-attractive. A solution which is both uniformly Lyapunov-stable and uniformly Lyapunov-attractive is called uniformly Lyapunov-asymptotically stable (see, e.g., SELL [166, p. 130]).

The concept of uniform asymptotically stability is a very strong form of stability in the sense of Lyapunov. It is easy to prove that any future attractive solution of (2.11) is uniformly Lyapunovasymptotically stable.

2.2.2 Theory of Autonomous Attractors and Repellers. There are many different notions of attractor and repeller for (autonomous) dynamical systems (see SIENZ [173] for a summary). Many authors use various properties such as irreducibility, topological transitivity or connectivity, in their definitions. As stated below, the concept of nonautonomous attractor and repeller in this thesis is closely related to the autonomous definitions used in CONLEY [52]. There, the main building blocks of attractor and repeller are invariance, compactness and local attractivity and repulsivity. We shortly review the definitions. Let $\phi: \mathbb{T} \times X \rightarrow X$ be a discrete (i.e., $\mathbb{T}=\mathbb{Z}$ ) or continuous (i.e., $\mathbb{T}=\mathbb{R}$ ) dynamical system on a metric space $(X, d)$. A compact set $A \subset X$ is called attractor of $\phi$ if $A$ is invariant, i.e.,

$$
\phi(t, A)=A \text { for all } t \in \mathbb{T},
$$

and if $A$ is the $\omega$-limit set of some neighborhood $V$ of $A$, i.e.,

$$
A=\omega(V):=\bigcap_{t \geq 0} \overline{\phi([t, \infty), V)}
$$

An invariant and compact set $R \subset X$ is called repeller if it is the $\alpha$-limit set of some neighborhood $W$ of $R$, i.e.,

$$
R=\alpha(W):=\bigcap_{t \leq 0} \overline{\phi((-\infty, t], W)} .
$$

One easily verifies that the definitions of past (future, all-time, respectively) attractor and repeller are indeed proper generalizations of this concept of attractor and repeller. In case of finite-time attractors and repellers, the situation is more subtle, since, given $T>0$, not every attractor is a $(0, T)$-attractor. However, given an attractor $A$, there exists a $\tau>0$ such that for all $T \geq \tau, A$ is a $(0, T)$-attractor.

2.2.3 Theory of Nonautonomous Attractors. Since the 1990s, the attractivity of nonautonomous sets is intensively discussed. In particular, the notions of pullback attractor and forward 
attractor have been introduced (see, e.g., Cheban \& Kloeden \& Schmalfuss [40, 41] or KLOEDEN \& KELLER \& SCHMALFUSS [97]). Closely related to pullback attractors are the socalled random attractors (see, e.g., ARnold [5], Crauel \& Debussche \& Flandoli [55], CRAuel \& Flandoli [57] and SCHEnK-Hoppé [158]). The most general form of a pullback attractor (see, e.g., ARNOLD [5, Definition 9.3.1, p. 483]) coincides basically with the notion of the $\mathcal{M}$-past attractor as introduced in Definition 2.1.1 (i). In the literature, $\mathcal{M}$ is called the attraction universe. Global pullback attractors are considered often, e.g., in CHEBAN \& KLOEDEN $\&$ SChmalfuss [41, Definition 2.4]. In this case, the universe $\mathcal{M}$ is supposed to contain all fiber-wise constant and compact nonautonomous sets. The past attractor as introduced in Definition 2.1.1 (i), however, is a local form of a pullback attractor. Here, the universe contains a neighborhood of the attractor itself. Another form of a local pullback attractor is introduced in LANGA \& ROBINSON \& SUÁREZ [102, 104].

In contrast to pullback attractors, forward attractors play a minor role in the literature. Usually, only global forward attractors are considered (for an exception, see AULBACH \& RASMUSSEN $\&$ Siegmund [16, Definition 3.4]). The $\mathcal{M}$-future attractor of Definition 2.1 .4 (i) provides a very general form of a forward attractor. By choosing $\mathcal{M}$ as the set of all fiber-wise constant and compact nonautonomous sets, one obtains the usual definition of a global forward attractor. A local form of a forward attractor, however, is provided by the future attractor as introduced in Definition 2.1.4 (ii).

Apart from these classes of attractors, pullback and forward attractors which are allowed to be noncompact are introduced in Aulbach \& RASMussen \& Siegmund [16, Definition 3.4]. Instead to be compact, attractors of this type are supposed to be "compactly generated". This notion includes some classes of noncompact nonautonomous invariant manifolds (see AULBACH \& RASMUSSEN \& SIEGMUND [17, 18]), but is no proper generalization of a compact attractor, since a compact attractor is not compactly generated in general.

\subsection{BIFURCATION AND TRANSITION}

This section is devoted to the introduction of various nonautonomous concepts of bifurcation and transition based on the notions of attractivity and repulsivity from Section 2.1.

Throughout this section, let $\left(\theta: \mathbb{T} \times P \rightarrow P, \varphi_{\alpha}: D_{\alpha} \subset \mathbb{T} \times P \times X \rightarrow X\right), \alpha \in\left(\alpha^{-}, \alpha^{+}\right)$, be a family of nonautonomous dynamical systems with a base set $P$ and a locally compact metric space $(X, d)$.

2.3.1 Definitions. In addition to the four different time domains (past, future, all-time and finite-time), we also distinguish between bifurcations of radii of attraction and repulsion and transitions of attractors and repellers. Attractors transitions are studied in, e.g., MA \& WANG [113], or see KLOEDEN \& SIEGMUND [98], where also nonautonomous attractors are considered.

We begin with the definitions concerning the past of the system.

2.3.1 Definition (Past Bifurcation and Transition). Let $\alpha_{0} \in\left(\alpha^{-}, \alpha^{+}\right)$. We say, $\left(\theta, \varphi_{\alpha}\right)$ admits a supercritical past bifurcation at $\alpha_{0}$ if there exists an $\hat{\alpha}>\alpha_{0}$ and a continuous function $\mu: D \subset \mathcal{O}(p) \times\left(\alpha_{0}, \hat{\alpha}\right) \rightarrow X$ such that one of the following two statements is fulfilled: 
(i) $\mu(\cdot, \alpha)$ is a past attractive solution of $\left(\theta, \varphi_{\alpha}\right)$ for all $\alpha \in\left(\alpha_{0}, \hat{\alpha}\right)$, and

$$
\lim _{\alpha \searrow \alpha_{0}} \mathfrak{A}_{\mu(\cdot, \alpha)}^{\leftarrow}=0
$$

is fulfilled. In case $X$ is a Banach space, we call this bifurcation total if

$$
\lim _{\alpha \triangleleft \alpha_{0}} d\left(\mathcal{A}_{\mu(\cdot, \alpha)}^{\leftarrow} \mid\{0\}\right)=0
$$

holds, otherwise, we call this bifurcation partial.

(ii) $\mu(\cdot, \alpha)$ is a past repulsive solution of $\left(\theta, \varphi_{\alpha}\right)$ for all $\alpha \in\left(\alpha_{0}, \hat{\alpha}\right)$, and

$$
\lim _{\alpha \searrow \alpha_{0}} \mathfrak{R}_{\mu(\cdot, \alpha)}^{\leftarrow}=0
$$

is fulfilled. In case $X$ is a Banach space, we call this bifurcation total if

$$
\lim _{\alpha \triangleright \alpha_{0}} d\left(\mathcal{R}_{\mu(\cdot, \alpha)}^{\leftarrow} \mid\{0\}\right)=0
$$

holds, otherwise, we call this bifurcation partial.

We say, $\left(\theta, \varphi_{\alpha}\right)$ admits a supercritical past attractor (past repeller, respectively) transition at $\alpha_{0}$ if there exist an $\hat{\alpha}>\alpha_{0}$ and past attractors (past repellers, respectively) $M_{\alpha}$ of $\left(\theta, \varphi_{\alpha}\right)$ for $\alpha \in\left(\alpha_{0}, \hat{\alpha}\right)$ with

$$
\lim _{\alpha \unlhd \alpha_{0}} \limsup _{t \rightarrow \infty} \operatorname{diam} M_{\alpha}\left(\theta_{-t} p\right)=0 \quad \text { for all } p \in P
$$

Accordingly, subcritical past bifurcations and past attractor (past repeller, respectively) transitions are defined by considering the limit $\alpha>\alpha_{0}$.

The following definition is devoted to the definition of future bifurcations and transitions.

2.3.2 Definition (Future Bifurcation And Transition). Let $\alpha_{0} \in\left(\alpha^{-}, \alpha^{+}\right)$. We say, $\left(\theta, \varphi_{\alpha}\right)$ admits a supercritical future bifurcation at $\alpha_{0}$ if there exists an $\hat{\alpha}>\alpha_{0}$ and a continuous function $\mu: D \subset \mathcal{O}(p) \times\left(\alpha_{0}, \hat{\alpha}\right) \rightarrow X$ such that one of the following two statements is fulfilled:

(i) $\mu(\cdot, \alpha)$ is a future attractive solution of $\left(\theta, \varphi_{\alpha}\right)$ for all $\alpha \in\left(\alpha_{0}, \hat{\alpha}\right)$, and

$$
\lim _{\alpha \searrow \alpha_{0}} \mathfrak{A}_{\mu(\cdot, \alpha)}=0
$$

is fulfilled. In case $X$ is a Banach space, we call this bifurcation total if

$$
\lim _{\alpha \searrow \alpha_{0}} d\left(\mathcal{A}_{\mu(\cdot, \alpha)} \mid\{0\}\right)=0
$$

holds, otherwise, we call this bifurcation partial.

(ii) $\mu(\cdot, \alpha)$ is a future repulsive solution of $\left(\theta, \varphi_{\alpha}\right)$ for all $\alpha \in\left(\alpha_{0}, \hat{\alpha}\right)$, and 


$$
\lim _{\alpha \searrow \alpha_{0}} \mathfrak{R}_{\mu(\cdot, \alpha)}=0
$$

is fulfilled. In case $X$ is a Banach space, we call this bifurcation total if

$$
\lim _{\alpha \searrow \alpha_{0}} d\left(\mathcal{R}_{\mu(\cdot, \alpha)} \mid\{0\}\right)=0
$$

holds, otherwise, we call this bifurcation partial.

We say, $\left(\theta, \varphi_{\alpha}\right)$ admits a supercritical future attractor (future repeller, respectively) transition at $\alpha_{0}$ if there exist an $\hat{\alpha}>\alpha_{0}$ and future attractors (future repellers, respectively) $M_{\alpha}$ of $\left(\theta, \varphi_{\alpha}\right)$ for $\alpha \in\left(\alpha_{0}, \hat{\alpha}\right)$ with

$$
\lim _{\alpha \triangleleft \alpha_{0}} \limsup _{t \rightarrow \infty} \operatorname{diam} M_{\alpha}\left(\theta_{t} p\right)=0 \quad \text { for all } p \in P .
$$

Accordingly, subcritical future bifurcations and future attractor (future repeller, respectively) transitions are defined by considering the limit $\alpha>\alpha_{0}$.

In the next definition, the notions of all-time bifurcation and transition are explained.

2.3.3 Definition (All-Time Bifurcation And Transition). Let $\alpha_{0} \in\left(\alpha^{-}, \alpha^{+}\right)$. We say, $\left(\theta, \varphi_{\alpha}\right)$ admits a supercritical all-time bifurcation at $\alpha_{0}$ if there exists an $\hat{\alpha}>\alpha_{0}$ and a continuous function $\mu: \mathcal{O}(p) \times\left(\alpha_{0}, \hat{\alpha}\right) \rightarrow X$ such that one of the following two statements is fulfilled:

(i) $\mu(\cdot, \alpha)$ is an all-time attractive solution of $\left(\theta, \varphi_{\alpha}\right)$ for all $\alpha \in\left(\alpha_{0}, \hat{\alpha}\right)$, and

$$
\lim _{\alpha \searrow \alpha_{0}} \mathfrak{A}_{\mu(\cdot, \alpha)}^{\leftrightarrow}=0
$$

is fulfilled. In case $X$ is a Banach space, we call this bifurcation total if

$$
\lim _{\alpha \unlhd \alpha_{0}} d\left(\mathcal{A}_{\mu(\cdot, \alpha)}^{\leftrightarrow} \mid\{0\}\right)=0
$$

holds, otherwise, we call this bifurcation partial.

(ii) $\mu(\cdot, \alpha)$ is an all-time repulsive solution of $\left(\theta, \varphi_{\alpha}\right)$ for all $\alpha \in\left(\alpha_{0}, \hat{\alpha}\right)$, and

$$
\lim _{\alpha \unlhd \alpha_{0}} \mathfrak{R}_{\mu(\cdot, \alpha)}^{\leftrightarrow}=0
$$

is fulfilled. In case $X$ is a Banach space, we call this bifurcation total if

$$
\lim _{\alpha \searrow \alpha_{0}} d\left(\mathcal{R}_{\mu(\cdot, \alpha)}^{\leftrightarrow} \mid\{0\}\right)=0
$$

holds, otherwise, we call this bifurcation partial.

We say, $\left(\theta, \varphi_{\alpha}\right)$ admits a supercritical all-time attractor (all-time repeller, respectively) transition at $\alpha_{0}$ if there exist an $\hat{\alpha}>\alpha_{0}$ and all-time attractors (all-time repellers, respectively) $M_{\alpha}$ of $\left(\theta, \varphi_{\alpha}\right)$ for $\alpha \in\left(\alpha_{0}, \hat{\alpha}\right)$ with 


$$
\lim _{\alpha \searrow \alpha_{0}} \sup _{p \in P} \operatorname{diam} M_{\alpha}(p)=0 .
$$

Accordingly, subcritical all-time bifurcations and all-time attractor (all-time repeller, respectively) transitions are defined by considering the limit $\alpha>\alpha_{0}$.

Finally, the following definition treats the concept of finite-time bifurcation and transition.

2.3.4 Definition (Finite-Time Bifurcation And Transition). Let $\alpha_{0} \in\left(\alpha^{-}, \alpha^{+}\right)$. We say, $\left(\theta, \varphi_{\alpha}\right)$ admits a supercritical $(p, T)$-bifurcation at $\alpha_{0}$ if there exists an $\hat{\alpha}>\alpha_{0}$ and a continuous function $\mu: \mathcal{O}^{T}(p) \times\left(\alpha_{0}, \hat{\alpha}\right) \rightarrow X$ such that one of the following two statements is fulfilled:

(i) $\mu(\cdot, \alpha)$ is a $(p, T)$-attractive solution of $\left(\theta, \varphi_{\alpha}\right)$ for all $\alpha \in\left(\alpha_{0}, \hat{\alpha}\right)$, and

$$
\lim _{\alpha \unlhd \alpha_{0}} \mathfrak{A}_{\mu(\cdot, \alpha)}^{(p, T)}=0
$$

is fulfilled.

(ii) $\mu(\cdot, \alpha)$ is a $(p, T)$-repulsive solution of $\left(\theta, \varphi_{\alpha}\right)$ for all $\alpha \in\left(\alpha_{0}, \hat{\alpha}\right)$, and

$$
\lim _{\alpha \searrow \alpha_{0}} \mathfrak{R}_{\mu(\cdot, \alpha)}^{(p, T)}=0
$$

holds.

We say, $\left(\theta, \varphi_{\alpha}\right)$ admits a supercritical $(p, T)$-attractor $((p, T)$-repeller, respectively) transition at $\alpha_{0} \in\left(\alpha^{-}, \alpha^{+}\right)$if there exist an $\hat{\alpha}>\alpha_{0}$ and $(p, T)$-attractors $((p, T)$-repellers, respectively) $M_{\alpha}$ of $\left(\theta, \varphi_{\alpha}\right)$ for $\alpha \in\left(\alpha_{0}, \hat{\alpha}\right)$ with

$$
\lim _{\alpha \searrow \alpha_{0}} \operatorname{diam} M_{\alpha}(p)=0
$$

Accordingly, subcritical $(p, T)$-bifurcations and $(p, T)$-attractor $((p, T)$-repeller, respectively) transitions are defined by considering the limit $\alpha>\alpha_{0}$.

2.3.2 Examples. In this subsection, two nonautonomous differential equations are discussed which are closely related to standard examples of equations admitting an autonomous bifurcation. The first example is of pitchfork type and leads to a total nonautonomous bifurcation; the second one is of transcritical type and gives rise to a partial nonautonomous bifurcation.

2.3.5 Example (Nonautonomous Pitchfork Bifurcation). We consider the nonautonomous differential equation

$$
\dot{x}=\alpha a(t) x+b(t) x^{3}=x\left(\alpha a(t)+b(t) x^{2}\right)
$$

depending on a real parameter $\alpha$ with continuous functions $a: \mathbb{R} \rightarrow \mathbb{R}$ and $b: \mathbb{R} \rightarrow \mathbb{R}_{\kappa}^{+}$for some $\kappa>0$. The ODE (2.12) is a nonautonomous version of the well-known autonomous differential equation

$$
\dot{x}=\alpha x+x^{3}=x\left(\alpha+x^{2}\right),
$$


which admits a pitchfork bifurcation (see, e.g., GUCKENHEIMER \& HOLMES [71, p. 150]). For fixed $\alpha \in \mathbb{R}$, (2.12) has already been discussed in Example 2.1.21, where we have derived sufficient conditions concerning the attractivity and repulsivity of the trivial solution. The following statements are direct consequences of these observations. The above nonautonomous differential equation admits a

- total supercritical past bifurcation at $\alpha=0$ if

$$
\liminf _{t \rightarrow-\infty}-\frac{a(t)}{b(t)}>0 \quad \text { and } \quad \limsup _{t \rightarrow-\infty}-\frac{a(t)}{b(t)}<\infty
$$

- total subcritical past bifurcation at $\alpha=0$ if

$$
\liminf _{t \rightarrow-\infty} \frac{a(t)}{b(t)}>0 \quad \text { and } \quad \limsup _{t \rightarrow-\infty} \frac{a(t)}{b(t)}<\infty
$$

- total supercritical future bifurcation at $\alpha=0$ if

$$
\liminf _{t \rightarrow \infty}-\frac{a(t)}{b(t)}>0 \text { and } \quad \limsup _{t \rightarrow \infty}-\frac{a(t)}{b(t)}<\infty
$$

- total subcritical future bifurcation at $\alpha=0$ if

$$
\liminf _{t \rightarrow \infty} \frac{a(t)}{b(t)}>0 \quad \text { and } \quad \limsup _{t \rightarrow \infty} \frac{a(t)}{b(t)}<\infty,
$$

- total supercritical all-time bifurcation at $\alpha=0$ if

$$
\inf _{t \in \mathbb{R}}-\frac{a(t)}{b(t)}>0 \quad \text { and } \quad \sup _{t \in \mathbb{R}}-\frac{a(t)}{b(t)}<\infty,
$$

- total subcritical all-time bifurcation at $\alpha=0$ if

$$
\inf _{t \in \mathbb{R}} \frac{a(t)}{b(t)}>0 \quad \text { and } \quad \sup _{t \in \mathbb{R}} \frac{a(t)}{b(t)}<\infty,
$$

- total supercritical $(\tau, T)$-bifurcation at $\alpha=0$ if

$$
-\frac{a(t)}{b(t)}>0 \quad \text { for all } t \in[\tau, \tau+T]
$$

- total subcritical $(\tau, T)$-bifurcation at $\alpha=0$ if

$$
\frac{a(t)}{b(t)}>0 \quad \text { for all } t \in[\tau, \tau+T]
$$

A generalization of this ODE is discussed in Section 6.2. It is also shown there that this example admits attractor and repeller transitions.

2.3.6 REMARK. A special form of the above example (for constant functions $a$ ) is discussed in LANGa \& Robinson \& SuÁrez [102, Proposition 3.1] and Caraballo \& Langa [37, Subsection 4.1]. 
2.3.7 Example (Nonautonomous Transcritical Bifurcation). We consider the nonautonomous differential equation

$$
\dot{x}=\alpha a(t) x+b(t) x^{2}=x(\alpha a(t)+b(t) x)
$$

depending on a real parameter $\alpha$ with continuous functions $a: \mathbb{R} \rightarrow \mathbb{R}$ and $b: \mathbb{R} \rightarrow \mathbb{R}_{\kappa}^{+}$for some $\kappa>0$. This ODE is a nonautonomous version of the well-known autonomous differential equation

$$
\dot{x}=\alpha x+x^{2}=x(\alpha+x),
$$

which admits a transcritical bifurcation (see HALE \& KOÇAK [77, Example 2.3, p. 28]). Analogously to Example 2.3.5, we see that the above nonautonomous differential equation admits a

- partial supercritical and subcritical past bifurcation at $\alpha=0$ if

$$
\liminf _{t \rightarrow-\infty}\left|\frac{a(t)}{b(t)}\right|>0 \text { and } \limsup _{t \rightarrow-\infty}\left|\frac{a(t)}{b(t)}\right|<\infty,
$$

- partial supercritical and subcritical future bifurcation at $\alpha=0$ if

$$
\liminf _{t \rightarrow \infty}\left|\frac{a(t)}{b(t)}\right|>0 \text { and } \quad \limsup _{t \rightarrow \infty}\left|\frac{a(t)}{b(t)}\right|<\infty,
$$

- partial supercritical and subcritical all-time bifurcation at $\alpha=0$ if

$$
\inf _{t \in \mathbb{R}}\left|\frac{a(t)}{b(t)}\right|>0 \quad \text { and } \quad \sup _{t \in \mathbb{R}}\left|\frac{a(t)}{b(t)}\right|<\infty,
$$

- partial supercritical and subcritical $(\tau, T)$-bifurcation at $\alpha=0$ if

$$
\left|\frac{a(t)}{b(t)}\right|>0 \quad \text { for all } t \in[\tau, \tau+T] .
$$

A generalization of this nonautonomous differential equation is discussed in Section 6.1.

\subsection{OThER Notions OF BIFURCATION AND TRANSITION}

In this section, several notions of bifurcation for (nonautonomous) dynamical systems are discussed with respect to their relationship to the concept of bifurcation and transition introduced in the previous section.

In the first subsection of this section, the autonomous bifurcation theory is treated. As mentioned in Section 1.2, the notion of the nonautonomous dynamical system is an abstraction of both topological skew product flows and random dynamical systems. In the recent studies of bifurcations of nonautonomous dynamical systems, one should also distinguish between topological skew product flows (cf. Subsection 2.4.2) and random dynamical systems (cf. Subsection 2.4.3). So far, there are only few approaches to the nonautonomous bifurcation theory without imposing special hypotheses on the base set $P$ such as compactness or existence of an invariant measure (cf. Subsection 2.4.4).

Please note that in Section 5.4, a relationship between the concept of finite-time bifurcation and the bifurcation theory of adiabatic systems is pointed out. 
2.4.1 Autonomous Bifurcation Theory. As mentioned in CHOW \& HALE [45] and MARSDEN \& HUGHES [115], there are two distinct aspects of autonomous bifurcation theory: static and dynamic. The static point of view is concentrated on the qualitative changes in the structure of the set of zeros of a function as parameters are varied. The dynamic bifurcation theory, however, is concerned with dynamical changes that occur in invariant sets (such as equilibria, periodic orbits, heteroclinic orbits and invariant tori).

Since the concept of bifurcation and transition used in this thesis is based on notions of attractivity and repulsivity, the static approach is too narrow in our situation, and we hope that the center manifold theory, which had been so fruitful in dynamic bifurcation theory, will be a method for future research in a higher dimensional nonautonomous bifurcation theory (cf. Example 7.2.8). It is not clear a priori to what extent the method of Lyapunov-Schmidt (see, e.g., HALE [75, Section 1]) is able to give a contribution in the nonautonomous context.

In the Introduction, an easy example already indicated that autonomous bifurcation phenomena can be described in terms of the concepts of nonautonomous bifurcation and transition. Please note also that in Chapter 6, one-dimensional nonautonomous bifurcations are studied, and Example 6.1.3 shows that the nonautonomous patterns are applicable also in the autonomous context. Moreover, it is shown in Chapter 7 that the classical bifurcation scenarios of saddle node, pitchfork, transcritical or Hopf type can be transferred to asymptotically autonomous equations. By regarding an autonomous system which admits a bifurcation of this type as an asymptotically autonomous system, one sees that the autonomous situation fits well into our context.

Interesting books on the topic of autonomous bifurcation theory are CHOW \& HALE [45], GUCKENHEIMER \& Holmes [71], HALE \& KoçAK [77], KuZneTSOW [100] and LuO \& WANG \& ZHU \& HAN [108]. For a brief introduction, see also CRAWFORD [59].

2.4.2 Bifurcation Theory of Topological Skew Product Flows. In the bifurcation theory of nonautonomous dynamical systems where the base set is supposed to have a certain topological structure, one distinguishes between attractor-repeller bifurcations and bifurcations of solutions.

An attractor-repeller bifurcation either occurs if a nontrivial attractor or repeller, respectively, shrinks down to a trivial object by variation of the parameter (this corresponds to the notion of transition), or if an attractor bifurcates from a repeller in the sense of Hausdorff distance. Please note that the attractors and repellers under consideration are autonomous objects of the skew product flow.

In Johnson \& MANTELlini [85], FABbRi \& Johnson \& MANTEllini [66] and FABbri \& JOHNSON [65], for one-dimensional nonautonomous differential equations with strictly ergodic time dependence (e.g., quasi-periodic equations are of this type), attractor-repeller bifurcations are considered. Bifurcations of attractors and repellers are also studied in JOHNSON \& KLOEDEN \& PAVANi [84] and Johnson [83] for deterministic counterparts of the Two-Step-BifurcationPattern. These considerations are based on the studies of Ludwig Arnold and his coworkers in the context of stochastic differential equations (see ARNOLD [5]). In GLENDINNING [69], a bifurcation of nonchaotic strange attractors of a quasi-periodic differential equation is verified, both numerically and analytically.

A bifurcation (of pitchfork and transcritical type) of almost periodic solutions of an almost periodic ordinary differential equation is examined in KLOEDEN [96].

We also mention autonomous bifurcations of invariant sets on which the dynamics of the system is nonperiodic, because the analysis requires - by means of the equation of perturbed motion- 
nonautonomous techniques. In JOHNSON [83] and JOHNSON \& YI [86], former studies concerning the bifurcation of invariant tori (see, e.g., SELL [168] and CHENCINER \& Iooss [42, 43]) are continued. The authors consider for an autonomous differential equation the loss of stability of an invariant set (which is, for instance, the closure of a nonperiodic and bounded trajectory). The bifurcation theory of tori with quasi-periodic flows whose frequencies satisfy the Diophantine condition is well-developed (see BROER \& HUITEMA \& TAKENS \& BRAAKSMA [36], BROER [35] and BRAAKSMA \& BROER [33]).

2.4.3 Bifurcation Theory of Random Dynamical Systems. To study bifurcation phenomena of random dynamical systems, two different concepts have been pursued so far: the socalled phenomenological approach (P-bifurcation) and the dynamical approach (D-bifurcation). For fundamental explanations and comparisons, we refer to ARNOLD \& NAMACHCHIVAYA \& SCHENK-Hoppé [8], ARnOld [5, 6] and SCHENK-Hoppé [155, 156] (see also SCHENK-Hoppé $[157,158])$.

P-bifurcations describe changes in stationary probability densities in special families of random dynamical systems. For instance, these densities exhibit transitions from one-peak to two-peak or crater-like structures. The concept of P-bifurcation can be formalized using the notion of equivalent probability densities, introduced by ZEEMAN [184, 185], which gives rise to a notion of structural stability. There are many drawbacks to this phenomenological approach, which are mentioned, e.g., in ARNOLD [5, Subsection 9.2.2]. Since P-bifurcations are static in the sense that there is no connection to stability properties obtained by Lyapunov exponents, we cannot expect a relationship to the concept of bifurcation introduced in this chapter.

Recently, the study of random bifurcation phenomena concentrated on D-bifurcations. A Dbifurcation occurs if from an invariant reference measure, another invariant measure bifurcates in the sense of weak convergence. It has been shown that this concept links the local bifurcation of invariant measures with the stability determined by the Lyapunov exponents.

2.4.4 Bifurcation Theory of General Nonautonomous Dynamical Systems. So far, there have been two approaches in the study of bifurcation phenomena of nonautonomous dynamical systems where no special hypotheses concerning the base set are made.

In KLOEDEN \& SiEgmund [98], a nonautonomous bifurcation is understood as a (continuous or discontinuous) transition from a nontrivial (global) pullback attractor to a trivial pullback attractor.

In LANGA \& ROBINSON \& SUÁREZ [102], for nonautonomous differential equations, notions of Lyapunov pullback-stable and Lyapunov pullback-unstable solutions are introduced, and bifurcations in form of merging processes of two distinct solutions with different stability behavior are studied by means of relatively simple examples. In their recent paper [104], the three authors found sufficient conditions for the Taylor coefficients of the right hand side of one-dimensional differential equations which guarantee the existence of such bifurcations. These conditions, however, are of a quite different form than the results obtained in Chapter 6 (cf. also the introduction of Chapter 6). 


\section{CHAPTER 3}

\section{NONAUTONOMOUS MORSE DECOMPOSITIONS}

The global asymptotic behavior of dynamical systems on compact metric spaces can be described via Morse decompositions. Their components, the so-called Morse sets, are obtained as intersections of attractors and repellers. In this chapter, nonautonomous generalizations of the Morse decomposition are established with respect to the notions of past and future attractivity and repulsivity. The dynamical properties of these decompositions are discussed, and nonautonomous Lyapunov functions which are constant on the Morse sets are constructed explicitly. Furthermore, Morse decompositions of one-dimensional and linear systems are analyzed.

For a discussion of elementary properties of Morse decompositions, we refer to the original work of CONLEy [52] and to RyBAKOwski [148, Chapter 3] (see also ColoniUs \& Kliemann [49, Appendix B2], RoBinson [145], AKIN [3] and ScHMidT [161]). Recently, OcHS [122] used the notion of weak attractor to construct Morse decompositions for random dynamical systems (see also CRAUEL \& DUC \& SiEgmund [56]).

In this chapter, we suppose that $(\theta: \mathbb{T} \times P \rightarrow P, \varphi: \mathbb{T} \times P \times X \rightarrow X)$ is an invertible NDS (this implies that $\mathbb{T}=\mathbb{R}$ or $\mathbb{T}=\mathbb{Z}$ ) with an arbitrary base set $P$ and a compact metric space $(X, d)$. Moreover, we assume that all (past or future) attractors and repellers $M$ under consideration fulfill either $P^{*}(M)=P$ or $M=\emptyset$.

Since Morse decompositions for the future are obtained via time reversal from Morse decompositions for the past, only the results concerning past Morse decompositions are proved in this chapter.

\subsection{Nonautonomous ATtractor-RePeller Pairs}

In this section, it is analyzed if for a given nonautonomous attractor, there exists a corresponding nonautonomous repeller and vice versa.

Due to the Axiom of Choice, there exists a set $P^{*} \subset P$ such that $[p] \cap P^{*}$ is a singleton for all $p \in P$. We write $P^{*}=P_{p}^{*} \cup P_{n}^{*}$ with $P_{p}^{*}$ containing all periodic points in $P^{*}$, i.e., $p^{*} \in P_{p}^{*}$ if and only if there exists a $\tau \in \mathbb{T}^{+}$with $p^{*}=\theta_{\tau} p^{*}$, and $P_{n}^{*}:=P^{*} \backslash P_{p}^{*}$.

Let $R$ be a past repeller. Due to Proposition 2.1.2 (vii), there exists an $\eta>0$ such that for all $p^{*} \in P^{*}$, there exists a $t^{*}\left(p^{*}\right)>0$ with

$$
\lim _{t \rightarrow \infty} d\left(\varphi\left(-t, \theta_{-\tau} p^{*}\right) U_{\eta}\left(R\left(\theta_{-\tau} p^{*}\right)\right) \mid R\left(\theta_{-\tau-t} p^{*}\right)\right)=0 \quad \text { for all } \tau \geq t^{*}\left(p^{*}\right) .
$$


For $\zeta \in(0, \eta]$, we define the compact nonautonomous set $B_{\zeta}$ by

$$
B_{\zeta}\left(\theta_{-t} p^{*}\right):=\left\{\begin{array}{cc}
X \backslash U_{\zeta}\left(R\left(\theta_{-t} p^{*}\right)\right) & : t \geq t^{*}\left(p^{*}\right) \\
X & : t<t^{*}\left(p^{*}\right)
\end{array} \quad \text { for all } p^{*} \in P_{n}^{*} \text { and } t \in \mathbb{T}\right.
$$

and

$$
B_{\zeta}\left(\theta_{-t} p^{*}\right):=X \backslash U_{\zeta}\left(R\left(\theta_{-t} p^{*}\right)\right) \text { for all } p^{*} \in P_{p}^{*} \text { and } t \in \mathbb{T} \text {. }
$$

3.1.1 Theorem (Existence of a Past AtTractor-RePeller Pair). Let $R$ be a past repeller, and set $\mathcal{M}:=\left\{B_{\zeta}: \zeta \in(0, \eta]\right\}$ with $B_{\zeta}$ defined as above. Then, there exists a uniquely determined $\mathcal{M}$-past attractor $R^{*} \subset B_{\eta}$, which is also a past attractor. Furthermore, $R^{*}$ is the maximal past attractor outside of $R$ in the following sense: Any other past attractor $A \supsetneq R^{*}$ has nonempty intersection with $R$. We call $\left(R^{*}, R\right)$ a past attractor-repeller pair.

Proof. We show that the hypotheses of Theorem 2.1.25 (i) are fulfilled by setting $B:=B_{\eta}$. Thereto, let $\zeta \in(0, \eta]$ and $p \in P$. In case $B(p)=X$, the condition (2.2) certainly holds, otherwise, there exist $p^{*} \in P^{*}$ and $\tau \geq t^{*}\left(p^{*}\right)$ with $\theta_{-\tau} p^{*}=p$. Due to (3.1), there exists a $\hat{t} \geq 0$ with

$$
d\left(\varphi(-t, p) U_{\eta}(R(p)) \mid R\left(\theta_{-t} p\right)\right)<\frac{\zeta}{2} \quad \text { for all } t \geq \hat{t} .
$$

This means that $\varphi(-t, p) U_{\eta}(R(p)) \subset U_{\frac{\zeta}{2}}\left(R\left(\theta_{-t} p\right)\right)$ for all $t \geq \hat{t}$. Thus, we have

$$
\varphi(-t, p) B_{\eta}(p)=X \backslash \varphi(-t, p) U_{\eta}(R(p)) \supset B_{\zeta}\left(\theta_{-t} p\right) \quad \text { for all } t \geq \hat{t}
$$

This implies the desired relation $\varphi\left(t, \theta_{-t} p\right) B_{\zeta}\left(\theta_{-t} p\right) \subset B(p)$ for all $t \geq \hat{t}$. Therefore, Theorem 2.1.25 (i) guarantees the existence of an $\mathcal{M}$-past attractor $R^{*} \subset B_{\eta}$. Due to Corollary 2.1.26, $R^{*}$ is also a past attractor. Let $A \supsetneq R^{*}$ be another past attractor. Then, there exists a $p \in P$ with $A(p) \supsetneq R^{*}(p)$. We choose an $x \in A(p) \backslash R^{*}(p)$. Since $A \ni(p, x)$ is a past attractor, there exists an $\tilde{\eta}>0$ with

$$
\lim _{t \rightarrow \infty} d\left(\varphi\left(t, \theta_{-t} p\right) U_{\tilde{\eta}}(\varphi(-t, p) x) \mid A(p)\right)=0 .
$$

Due to $\lim _{t \rightarrow \infty} d\left(\varphi(-t, p) x, R\left(\theta_{-t} p\right)\right)=0$ (we will see this in Theorem 3.1 .5 (ii)), there exists a sequence $\left\{y_{n}\right\}_{n \in \mathbb{N}}$ in $R(p)$ with

$$
\lim _{n \rightarrow \infty} d\left(y_{n}, A(p)\right)=0
$$

Since $R(p)$ and $A(p)$ are compact, this implies that their intersection is nonempty.

Based on Proposition 2.1.22, the construction of a future attractor-repeller pair is not difficult.

3.1.2 Corollary (Existence of a Future Attractor-Repeller Pair). Let $A$ be a future attractor. Then, there exists a uniquely determined future repeller $A^{*} \subset(P \times X) \backslash A$, which is the maximal future repeller outside of $A$ in the following sense: Any future repeller $R \supsetneq A^{*}$ has nonempty intersection with $A$. We call $\left(A, A^{*}\right)$ a future attractor-repeller pair.

Proof. Because of Proposition 2.1.22, $A$ is a past repeller of $(\theta, \varphi)^{-1}$. Due to Theorem 3.1.1, there exists a corresponding past attractor $A^{*}$ of $(\theta, \varphi)^{-1}$. Then, $A^{*}$ is a future repeller of $(\theta, \varphi)$. 
The property that $A^{*}$ is the maximal future repeller outside of $A$ follows easily from Theorem 3.1.1.

It is natural to ask if a past attractor implies the existence of a past repeller and, equivalently, if a future repeller implies the existence of a future attractor. The following example shows that this is not fulfilled.

3.1.3 EXAMPLE. The nonautonomous differential equation

$$
\dot{x}=f(t, x)
$$

with the function $f: \mathbb{R} \times[0,1] \rightarrow \mathbb{R}$, defined by

$$
f(t, x):=\left\{\begin{array}{cl}
|2 x-1|-1 & : t \geq 0 \text { and } x \in[0,1] \\
|2 x-1|-1 & : t<0 \text { and } x \in\left[0, \frac{1}{2}\right] \\
(2-2 t)\left(x-\frac{1}{2}\right)-1 & : t<0 \text { and } x \in\left[\frac{1}{2}, \frac{2-t}{2-2 t}\right], \\
0 & : t<0 \text { and } x \in\left(\frac{2-t}{2-2 t}, 1\right]
\end{array},\right.
$$

generates a nonautonomous dynamical system with $P=\mathbb{R}$ and $X=[0,1]$. The invariant nonautonomous set $A:=\mathbb{R} \times\{0\}$ is a past (as well as a future and an all-time) attractor. Assume, there exists a past repeller $A^{*} \subset \mathbb{R} \times(0,1]$. Due to the invariance of $A^{*}$, the form of the right hand side implies that there exist $\gamma>\frac{1}{2}$ and $\tau_{1}<0$ with

$$
A^{*}(t) \subset(\gamma, 1] \text { for all } t \leq \tau_{1} .
$$

Thus, there exists a $\tau_{2}<\tau_{1}$ with

$$
A^{*}(s)=A^{*}(t) \text { and } f(t,[\gamma, 1])=\{0\} \quad \text { for all } t, s \leq \tau_{2} .
$$

This contradicts the fact that $A^{*}$ is a past repeller.

3.1.4 REMARK. This example shows that there is no possibility to construct an all-time attractor-repeller pair: The past attractor $A$ is also an all-time attractor, and no corresponding all-time repeller exists, since this would be also a past repeller. Furthermore, $A$ is an all-time repeller for the system under time reversal (see Proposition 2.1.22), and there is no corresponding all-time attractor, since this would be an all-time repeller for the original system.

Now, some properties of nonautonomous attractor-repeller pairs are derived.

3.1.5 TheOREM (Properties of NonAutonomous ATtRaCtOR-REPELLER PAirs). Let $\left(R^{*}, R\right)$ be a past attractor-repeller pair. Then, the following statements are fulfilled:

(i) Past isolation. There exists a $\beta>0$ such that for all $p \in P$, there exists a $\tau>0$ with

$$
U_{\beta}\left(R^{*}\left(\theta_{-t} p\right)\right) \cap U_{\beta}\left(R\left(\theta_{-t} p\right)\right)=\emptyset \quad \text { for all } t \geq \tau .
$$

(ii) Backward convergence. Let $p \in P$ and $C \subset X \backslash R^{*}(p)$ be a compact set. Then, we have

$$
\lim _{t \rightarrow \infty} d\left(\varphi(-t, p) C \mid R\left(\theta_{-t} p\right)\right)=0 .
$$

(iii) Pullback convergence. For all $p \in P$ and all functions $\gamma: \mathbb{T}^{+} \rightarrow X$ with

$$
\liminf _{t \rightarrow \infty} d\left(\gamma(t), R\left(\theta_{-t} p\right)\right)>0
$$


we have

$$
\lim _{t \rightarrow \infty} d\left(\varphi\left(t, \theta_{-t} p\right) \gamma(t), R^{*}(p)\right)=0 .
$$

Let $\left(A, A^{*}\right)$ be a future attractor-repeller pair. Then, the following statements are fulfilled:

(i) Future isolation. There exists a $\beta>0$ such that for all $p \in P$, there exists a $\tau>0$ with

$$
U_{\beta}\left(A\left(\theta_{t} p\right)\right) \cap U_{\beta}\left(A^{*}\left(\theta_{t} p\right)\right)=\emptyset \quad \text { for all } t \geq \tau
$$

(ii) Forward convergence. Let $p \in P$ and $C \subset X \backslash A^{*}(p)$ be a compact set. Then, we have

$$
\lim _{t \rightarrow \infty} d\left(\varphi(t, p) C \mid A\left(\theta_{t} p\right)\right)=0 .
$$

(iii) Pushforward convergence. For all $p \in P$ and all functions $\gamma: \mathbb{T}^{+} \rightarrow X$ with

$$
\liminf _{t \rightarrow \infty} d\left(\gamma(t), A\left(\theta_{t} p\right)\right)>0
$$

we have

$$
\lim _{t \rightarrow \infty} d\left(\varphi\left(-t, \theta_{t} p\right) \gamma(t), A^{*}(p)\right)=0
$$

Proof. Let $\left(R^{*}, R\right)$ be a past attractor-repeller pair with $\eta$ and $\mathcal{M}$ defined as in the introduction of this section.

(i) Theorem 3.1.1 implies $R^{*} \subset B_{\eta}$. The assertion follows by choosing $\beta:=\frac{\eta}{2}$.

(ii) Let $p \in P$ and $C \subset X \backslash R^{*}(p)$ be a compact set. Since $R^{*}$ is an $\mathcal{M}$-past attractor and thus a $\left\{B_{\eta}\right\}$-past attractor, there exists a $\tau>0$ such that

$$
C \cap \varphi\left(t, \theta_{-t} p\right) B_{\eta}\left(\theta_{-t} p\right)=\emptyset \quad \text { for all } t \geq \tau \text {. }
$$

Hence, for all $t \geq \tau$, the relation $\varphi(-t, p) C \cap B_{\eta}\left(\theta_{-t} p\right)=\emptyset$ is fulfilled, and therefore, we have $\lim _{t \rightarrow \infty} d\left(\varphi(-t, p) C, R\left(\theta_{-t} p\right)\right)=0$.

(iii) We set $\zeta:=\frac{1}{2} \min \left\{\eta, \liminf _{t \rightarrow \infty} d\left(\gamma(t), R\left(\theta_{-t} p\right)\right)\right\}$ and see that there exists a $\tau>0$ with

$$
\gamma(t) \in B_{\zeta}\left(\theta_{-t} p\right) \text { for all } t \geq \tau \text {. }
$$

This finishes the proof, since $B_{\zeta} \in \mathcal{M}$ and $R^{*}$ is a $\mathcal{M}$-past attractor.

Theorem 3.1.1 implies that, given a past repeller $R$, the set $R^{*}$ is the uniquely determined past attractor outside of $R$ with the property of pullback convergence as described in Theorem 3.1.5 (iii). It is easy to see that such a uniqueness result is not valid for past repellers, i.e., it is possible that $\left(A, R_{1}\right)$ and $\left(A, R_{2}\right)$ are past attractor-repeller pairs with $R_{1} \neq R_{2}$. The following proposition says that in this case, $R_{1}$ and $R_{2}$ are converging to each other when time tends to the past.

\subsubsection{Proposition (Form of NONUNiQUENESS OF NONAUTONOMOUS ATTRACTOR-} REPELler PAIRS). Let $R_{1}$ and $R_{2}$ be past repellers with $R_{1}^{*}=R_{2}^{*}$. Then, we have

$$
\lim _{t \rightarrow \infty} d_{H}\left(R_{1}\left(\theta_{-t} p\right), R_{2}\left(\theta_{-t} p\right)\right)=0 \quad \text { for all } p \in P .
$$

Let $A_{1}$ and $A_{2}$ be future attractors with $A_{1}^{*}=A_{2}^{*}$. Then, we have 


$$
\lim _{t \rightarrow \infty} d_{H}\left(A_{1}\left(\theta_{t} p\right), A_{2}\left(\theta_{t} p\right)\right)=0 \quad \text { for all } p \in P
$$

Proof. Suppose, there exist a $p \in P$ and sequences $\left\{t_{n}\right\}_{n \in \mathbb{N}}$ in $\mathbb{T}$ and $\left\{\gamma_{n}\right\}_{n \in \mathbb{N}}$ in $X$ with $\lim _{n \rightarrow \infty} t_{n}=\infty$ and $\gamma_{n} \in R_{1}\left(\theta_{-t_{n}} p\right)$ such that

$$
\liminf _{n \rightarrow \infty} d\left(\gamma_{n}, R_{2}\left(\theta_{-t_{n}} p\right)\right)>0
$$

Hence, Theorem 3.1.5 (iii), applied to the attractor-repeller pair $\left(R_{2}^{*}, R_{2}\right)$, implies that

$$
\lim _{n \rightarrow \infty} d\left(\varphi\left(t_{n}, \theta_{-t_{n}} p\right) \gamma_{n}, R_{2}^{*}(p)\right)=0
$$

Since $\varphi\left(t_{n}, \theta_{-t_{n}} p\right) \gamma_{n} \in R_{1}(p)$ and $R_{1}$ and $R_{1}^{*}=R_{2}^{*}$ are compact nonautonomous sets, we obtain $R_{1}(p) \cap R_{1}^{*}(p) \neq \emptyset$. This is a contradiction.

\subsection{Nonautonomous Morse Decompositions}

In this section, the notion of the attractor-repeller pair is generalized by considering Morse decompositions.

\subsubsection{Definition (Nonautonomous Morse Decompositions). A family} $\left\{M_{1}, M_{2}, \ldots, M_{n}\right\}$ of nonautonomous sets, the so-called Morse sets, is called past Morse decomposition if the representation

$$
M_{i}=R_{i}^{*} \cap R_{i-1} \quad \text { for all } i \in\{1, \ldots, n\}
$$

holds with past repellers

$$
P \times X=R_{0} \supsetneq R_{1} \supsetneq \cdots \supsetneq R_{n}=\emptyset
$$

fulfilling $\emptyset=R_{0}^{*} \subsetneq R_{1}^{*} \subsetneq \cdots \subsetneq R_{n}^{*}=P \times X$.

A family $\left\{M_{1}, M_{2}, \ldots, M_{n}\right\}$ of nonautonomous sets, the so-called Morse sets, is called future Morse decomposition if the representation

$$
M_{i}=A_{i} \cap A_{i-1}^{*} \quad \text { for all } i \in\{1, \ldots, n\}
$$

holds with future attractors

$$
\emptyset=A_{0} \subsetneq A_{1} \subsetneq \cdots \subsetneq A_{n}=P \times X
$$

fulfilling $P \times X=A_{0}^{*} \supsetneq A_{1}^{*} \supsetneq \cdots \supsetneq A_{n}^{*}=\emptyset$.

3.2.2 REMARK. Let $(A, R)$ be a past (future, respectively) attractor-repeller pair such that the relation $\emptyset \subsetneq A \subsetneq P \times X$ is fulfilled. Then, $\{A, R\}$ is a past (future, respectively) Morse decomposition. 


\subsubsection{Proposition (BAsic Properties of Nonautonomous Morse Decomposi-} TIONS). The Morse sets of a past Morse decomposition $\left\{M_{1}, \ldots, M_{n}\right\}$ are nonempty, invariant, pairwise disjoint and past isolated, i.e., there exists a $\beta>0$ such that for all $1 \leq i<j \leq n$ and $p \in P$, there exists a $\tau>0$ with

$$
U_{\beta}\left(M_{i}\left(\theta_{-t} p\right)\right) \cap U_{\beta}\left(M_{j}\left(\theta_{-t} p\right)\right)=\emptyset \quad \text { for all } t \geq \tau .
$$

The Morse sets of a future Morse decomposition $\left\{M_{1}, \ldots, M_{n}\right\}$ are nonempty, invariant, pairwise disjoint and future isolated, i.e., there exists a $\beta>0$ such that for all $1 \leq i<j \leq n$ and $p \in P$, there exists a $\tau>0$ with

$$
U_{\beta}\left(M_{i}\left(\theta_{t} p\right)\right) \cap U_{\beta}\left(M_{j}\left(\theta_{t} p\right)\right)=\emptyset \quad \text { for all } t \geq \tau .
$$

Proof. Let $M_{i}=R_{i}^{*} \cap R_{i-1}$ be a Morse set. Since $R_{i-1}^{*} \subsetneq R_{i}^{*}$, we can choose a $p \in P$ and an $x \in R_{i}^{*}(p) \backslash R_{i-1}^{*}(p)$. Since $R_{i}^{*} \ni(p, x)$ is a past attractor, there exists an $\eta>0$ with

$$
\lim _{t \rightarrow \infty} d\left(\varphi\left(t, \theta_{-t} p\right) U_{\eta}(\varphi(-t, p) x) \mid R_{i}^{*}(p)\right)=0
$$

Due to $\lim _{t \rightarrow \infty} d\left(\varphi(-t, p) x, R_{i-1}\left(\theta_{-t} p\right)\right)=0$ (cf. Theorem 3.1 .5 (ii)), this means that there exists a sequence $\left\{y_{n}\right\}_{n \in \mathbb{N}}$ in $R_{i-1}(p)$ with

$$
\lim _{n \rightarrow \infty} d\left(y_{n}, R_{i}^{*}(p)\right)=0 .
$$

Since $R_{i-1}(p)$ and $R_{i}^{*}(p)$ are compact, this implies $M_{i}=R_{i}^{*} \cap R_{i-1} \neq \emptyset$. Furthermore, $M_{i}$ is the intersection of two invariant nonautonomous sets and thus invariant. Choose another Morse set $M_{j}=R_{j}^{*} \cap R_{j-1}$. W.l.o.g, we assume $j>i$. Then, we get

$$
M_{i} \cap M_{j}=R_{i}^{*} \cap R_{i-1} \cap R_{j}^{*} \cap R_{j-1}=R_{i-1}^{*} \cap R_{j-1} \subset R_{j-1}^{*} \cap R_{j-1}=\emptyset .
$$

The fact that the Morse sets are past isolated is an easy consequence of Theorem 3.1.5 (i).

As in the autonomous case, nonautonomous Morse decompositions are not uniquely determined.

3.2.4 Definition. We say, the past Morse decomposition $\left\{M_{1}, \ldots, M_{n}\right\}$ is finer than the past Morse decomposition $\left\{\tilde{M}_{1}, \ldots, \tilde{M}_{m}\right\}$ if

$$
\lim _{t \rightarrow \infty} d\left(\bigcup_{i=1}^{n} M_{i}\left(\theta_{-t} p\right) \mid \bigcup_{i=1}^{m} \tilde{M}_{i}\left(\theta_{-t} p\right)\right)=0 \quad \text { for all } p \in P
$$

is fulfilled.

We say, the future Morse decomposition $\left\{M_{1}, \ldots, M_{n}\right\}$ is finer than the future Morse decomposition $\left\{\tilde{M}_{1}, \ldots, \tilde{M}_{m}\right\}$ if

$$
\lim _{t \rightarrow \infty} d\left(\bigcup_{i=1}^{n} M_{i}\left(\theta_{t} p\right) \mid \bigcup_{i=1}^{m} \tilde{M}_{i}\left(\theta_{t} p\right)\right)=0 \quad \text { for all } p \in P
$$

is fulfilled. 


\subsubsection{REMARKS.}

(i) The above definition is a generalization of the notion of a finer (autonomous) Morse decomposition. In the autonomous case, a Morse decomposition $\left\{M_{1}, \ldots, M_{n}\right\}$ is called finer than the Morse decomposition $\left\{\tilde{M}_{1}, \ldots, \tilde{M}_{m}\right\}$ if for all $j \in\{1, \ldots, m\}$, there exists an $i \in\{1, \ldots, n\}$ such that $M_{i} \subset \tilde{M}_{j}$ (see, e.g., Colonius \& KLIEMAnN [49, p. 542]). It is easy to see that this is equivalent to

$$
d\left(\bigcup_{i=1}^{n} M_{i} \mid \bigcup_{i=1}^{m} \tilde{M}_{i}\right)=0
$$

The additional limit in our nonautonomous context is motivated by Proposition 3.1.6.

(ii) There are different forms of nonuniqueness for the Morse sets. As seen in Proposition 3.1.6, two past attractor-repeller pairs are converging to each other in case the past attractors are equal. One can find examples to show that such a (weak) form of nonuniqueness is not valid for arbitrary Morse decompositions (i.e., those consisting of more than two sets). However, in the special cases of one-dimensional and linear systems (cf. Section 3.4 and 3.5), one obtains similar results as in Proposition 3.1.6 (cf. Proposition 3.4.2 and 3.5.5).

The following theorem shows that Morse sets are important for the asymptotic behavior of nonautonomous dynamical systems.

\subsubsection{Theorem (Dynamical Properties of Nonautonomous Morse Decompo-} SiTIONS). Pullback convergence. Let $\left\{M_{1}, \ldots, M_{n}\right\}$ be a past Morse decomposition obtained by the finite sequence of past repellers $R_{0} \supset \cdots \supset R_{n}$. Then, for all $p \in P$ and all functions $\gamma: \mathbb{T}^{+} \rightarrow X$ with

$$
\liminf _{t \rightarrow \infty} d\left(\gamma(t), \bigcup_{j=1}^{n} \partial R_{j}\left(\theta_{-t} p\right)\right)>0,
$$

we have

$$
\lim _{t \rightarrow \infty} d\left(\varphi\left(t, \theta_{-t} p\right) \gamma(t), \bigcup_{j=1}^{n} M_{j}(p)\right)=0 .
$$

Pushforward convergence. Let $\left\{M_{1}, \ldots, M_{n}\right\}$ be a future Morse decomposition obtained by the finite sequence of future attractors $A_{0} \subset \cdots \subset A_{n}$. Then, for all $p \in P$ and all functions $\gamma: \mathbb{T}^{+} \rightarrow X$ with

$$
\liminf _{t \rightarrow \infty} d\left(\gamma(t), \bigcup_{j=1}^{n} \partial A_{j}\left(\theta_{t} p\right)\right)>0,
$$

we have

$$
\lim _{t \rightarrow \infty} d\left(\varphi\left(-t, \theta_{t} p\right) \gamma(t), \bigcup_{j=1}^{n} M_{j}(p)\right)=0
$$

Proof. We assume w.l.o.g. that there exists an $i \in\{1, \ldots, n\}$ with

$$
\gamma(t) \in R_{i-1}\left(\theta_{-t} p\right) \quad \text { and } \quad \gamma(t) \notin R_{i}\left(\theta_{-t} p\right) \quad \text { for all } t>0 \text {. }
$$


Then, $\liminf _{t \rightarrow \infty} d\left(\gamma(t), \partial R_{i}\left(\theta_{-t} p\right)\right)>0$ yields $\liminf _{t \rightarrow \infty} d\left(\gamma(t), R_{i}\left(\theta_{-t} p\right)\right)>0$. Therefore, Theorem 3.1.5 implies that

$$
\lim _{t \rightarrow \infty} d\left(\varphi\left(t, \theta_{-t} p\right) \gamma(t), R_{i}^{*}(p)\right)=0 .
$$

Assume, there exist an $\varepsilon>0$ and a sequence $\left\{t_{n}\right\}_{n \in \mathbb{N}}$ in $\mathbb{T}^{+}$with $\lim _{n \rightarrow \infty} t_{n}=\infty$ and

$$
d\left(\varphi\left(t_{n}, \theta_{-t_{n}} p\right) \gamma\left(t_{n}\right), M_{i}(p)\right) \geq \varepsilon \quad \text { for all } n \in \mathbb{N} .
$$

W.l.o.g., the sequence $\left\{\varphi\left(t_{n}, \theta_{-t_{n}} p\right) \gamma\left(t_{n}\right)\right\}_{n \in \mathbb{N}}$ in $R_{i-1}(p)$ is convergent with limit $x_{0} \in R_{i-1}(p)$ $\left(R_{i-1}(p)\right.$ is compact). Moreover, $x_{0} \in R_{i}^{*}(p)$, since (3.2) holds and $R_{i}^{*}(p)$ is compact. Thus, $x_{0} \in M_{i}(p)=R_{i}^{*}(p) \cap R_{i-1}(p)$. This contradicts (3.3) and finishes the proof of this theorem.

3.2.7 REMARK. In contrast to attractor-repeller pairs, backward and forward convergence conditions as described in Theorem 3.1.5 do not hold for arbitrary Morse decompositions. However, in the special cases of one-dimensional and linear systems (cf. Section 3.4 and 3.5), one obtains similar results as in Theorem 3.1.5 (cf. Theorem 3.4.1 and 3.5.4).

If the backward (in case of a past Morse decomposition) or forward (in case of a future Morse decomposition) convergence holds, the following uniqueness result concerning the past attractors or future repellers, respectively, is fulfilled.

3.2.8 Proposition. Let $\left\{M_{1}, \ldots, M_{n}\right\}$ be a past Morse decomposition obtained by the finite sequence of past repellers $R_{0} \supset \cdots \supset R_{n}$. We assume that the backward convergence holds, i.e., for all $(p, x) \in P \times X$, there exists an $i \in\{1, \ldots, n\}$ with

$$
\lim _{t \rightarrow \infty} d\left(\varphi(-t, p) x, M_{i}\left(\theta_{-t} p\right)\right)=0 .
$$

Then, the representation

$$
R_{i}^{*}=\left\{(p, x): \lim _{t \rightarrow \infty} d\left(\varphi(-t, p) x, \bigcup_{j=1}^{i} M_{j}\left(\theta_{-t} p\right)\right)=0\right\} \quad \text { for all } i \in\{1, \ldots, n\}
$$

holds, i.e., the past attractors of the past Morse decomposition are uniquely determined. Let $\left\{M_{1}, \ldots, M_{n}\right\}$ be a future Morse decomposition obtained by the finite sequence of future attractors $A_{0} \subset \cdots \subset A_{n}$. We assume that the forward convergence holds, i.e., for all $(p, x) \in P \times X$, there exists an $i \in\{1, \ldots, n\}$ with

$$
\lim _{t \rightarrow \infty} d\left(\varphi(t, p) x, M_{i}\left(\theta_{t} p\right)\right)=0 .
$$

Then, the representation

$$
A_{i}^{*}=\left\{(p, x): \lim _{t \rightarrow \infty} d\left(\varphi(t, p) x, \bigcup_{j=i+1}^{n} M_{j}\left(\theta_{t} p\right)\right)=0\right\} \quad \text { for all } i \in\{1, \ldots, n\}
$$

holds, i.e., the future repellers of the future Morse decomposition are uniquely determined. 
Proof. $(\subseteq)$ Let $(p, x) \in R_{i}^{*}$. We choose $j \in\{1, \ldots, n\}$ such that

$$
0=\lim _{t \rightarrow \infty} d\left(\varphi(-t, p) x, M_{j}\left(\theta_{-t} p\right)\right)=\lim _{t \rightarrow \infty} d\left(\varphi(-t, p) x, R_{j-1}\left(\theta_{-t} p\right)\right) .
$$

The assumption $j>i$ leads to

$$
\lim _{t \rightarrow \infty} d\left(\varphi(-t, p) x, R_{i}\left(\theta_{-t} p\right)\right)=0 .
$$

This contradicts Theorem 3.1.5 (i), since $\varphi(-t, p) x \in R_{i}^{*}\left(\theta_{-t} p\right)$ for all $t \in \mathbb{T}$.

() Let $(p, x) \in(P \times X) \backslash R_{i}^{*}$. Then, Theorem 3.1.5 (ii) implies

$$
\lim _{t \rightarrow \infty} d\left(\varphi(-t, p) x, R_{i}\left(\theta_{-t} p\right)\right)=0 .
$$

The assumption

$$
\lim _{t \rightarrow \infty} d\left(\varphi(-t, p) x, \bigcup_{j=1}^{i} M_{j}\left(\theta_{-t} p\right)\right)=0
$$

leads to

$$
\lim _{t \rightarrow \infty} d\left(\varphi(-t, p) x, R_{i}^{*}\left(\theta_{-t} p\right)\right)=0,
$$

since $M_{j} \subset R_{i}^{*}$ for $j \in\{1, \ldots, i\}$. Because of Theorem 3.1.5 (i), this is a contradiction to (3.4).

\subsection{LyapunOV FunCtions FOR NONAUTONOMOUS MORSE DECOMPOSITIONS}

In this section, nonautonomous Lyapunov functions which are constant on the Morse sets and which strictly decrease outside them are obtained explicitly. A similar construction is used in CONLEY [52, §5 and §6 of Chapter II] (see also Franks [68, §1], Robinson [145, Chapter X] and NORTON [121]), and this technique has also been adapted in KLOEDEN [93, 94] and ARNOLD \& SCHMALFUSS [7] in the nonautonomous setting.

First, the case that the nonautonomous Morse decomposition is given by a nonautonomous attractor-repeller pair is treated.

3.3.1 LEMMA. Let $(A, R)$ be a past (future, respectively) attractor-repeller pair. Then, there exists a function $L: P \times X \rightarrow[0,1]$ which is continuous with respect to $x \in X$ such that $\left.L\right|_{A} \equiv 0,\left.L\right|_{R} \equiv 1$ and

$$
L\left(\theta_{t} p, \varphi(t, p) x\right)<L(p, x) \quad \text { for all } t>0 \text { and }(p, x) \in(P \times X) \backslash(A \cup R)
$$

is satisfied.

PROOF. In case of a past attractor-repeller pair, $R^{*}=A$ is fulfilled, and we define the function $V: P \times X \rightarrow[0,1]$ by

$$
V(p, x):=\frac{d\left(x, R^{*}(p)\right)}{d\left(x, R^{*}(p)\right)+d(x, R(p))} \quad \text { for all }(p, x) \in P \times X .
$$


This function is continuous with respect to $x \in X$ and fulfills $\left.V\right|_{R^{*}} \equiv 0,\left.V\right|_{R} \equiv 1$, but is not necessarily decreasing along solutions. Therefore, we define by

$$
V^{*}(p, x):=\inf _{s \geq 0} V\left(\theta_{-s} p, \varphi(-s, p) x\right) \text { for all }(p, x) \in P \times X
$$

a function $V^{*}: P \times X \rightarrow[0,1]$, which obviously satisfies $\left.V^{*}\right|_{R^{*}} \equiv 0,\left.V^{*}\right|_{R} \equiv 1$ and $V^{*}\left(\theta_{t} p, \varphi(t, p) x\right) \leq V^{*}(p, x)$ for all $t \geq 0$ and $(p, x) \in P \times X$. To prove that $V^{*}(p, \cdot)$ is continuous for all $p \in P$, we first choose $\xi \in X \backslash R^{*}(p)$ and $\varepsilon>0$. Then, there exists a $\hat{\delta}>0$ such that $C:=\overline{U_{\hat{\delta}}(\xi)} \subset X \backslash R^{*}(p)$. It follows that

$$
\lim _{s \rightarrow \infty} \inf _{x \in C} V\left(\theta_{-s} p, \varphi(-s, p) x\right)=1,
$$

since $\lim _{s \rightarrow \infty} d\left(\varphi(-s, p) C, R\left(\theta_{-s} p\right)\right)=0$ (cf. Theorem 3.1.5 (ii)) and there exist a $\beta>0$ and an $\hat{s}>0$ such that $d\left(\varphi(-s, p) C, R^{*}\left(\theta_{-s} p\right)\right) \geq \frac{\beta}{2}$ for all $s \geq \hat{s}$ (cf. Theorem 3.1.5 (i), (ii)). Thus, there exists an $s_{0}>0$ such that

$$
\inf _{x \in C} V\left(\theta_{-s} p, \varphi(-s, p) x\right)>1-\varepsilon \quad \text { for all } s \geq s_{0} .
$$

Due to the continuity of $V(\theta(\cdot, p), \varphi(\cdot, p, \cdot)): \mathbb{T} \times X \rightarrow \mathbb{R}$, there exists a $\delta \in(0, \hat{\delta})$ such that

$$
\left|V\left(\theta_{-s} p, \varphi(-s, p) \xi\right)-V\left(\theta_{-s} p, \varphi(-s, p) x\right)\right|<\varepsilon \quad \text { for all } x \in U_{\delta}(\xi) \text { and } 0 \leq s \leq s_{0} .
$$

This implies that $V^{*}(p, \cdot)$ is continuous in $\xi \notin R^{*}(p)$. The continuity of $V^{*}(p, \cdot)$ in $\xi \in R^{*}(p)$ follows directly from the continuity of $V$. Please note that $V$ is not strictly decreasing along solutions in $(P \times X) \backslash\left(R^{*} \cup R\right)$. Therefore, we define $L$ to be a weighted average of $V^{*}$ over the backward solution:

$$
L(p, x):=\int_{0}^{\infty} e^{-s} V^{*}\left(\theta_{-s} p, \varphi(-s, p) x\right) d s \text { for all }(p, x) \in P \times X .
$$

This function is obviously continuous with respect to $x \in X$, and we have

$$
\begin{aligned}
L\left(\theta_{t} p, \varphi(t, p, x)\right) & =\int_{0}^{\infty} e^{-s} V^{*}\left(\theta_{-s} \theta_{t} p, \varphi\left(-s, \theta_{t} p\right) \varphi(t, p) x\right) d s \\
& =\int_{0}^{\infty} e^{-s} V^{*}\left(\theta_{t} \theta_{-s} p, \varphi\left(t, \theta_{-s} p\right) \varphi(-s, p) x\right) d s \\
& \leq \int_{0}^{\infty} e^{-s} V^{*}\left(\theta_{-s} p, \varphi(-s, p) x\right) d s=L(p, x) .
\end{aligned}
$$

To prove that this function is also strictly decreasing along solutions in $(P \times X) \backslash\left(R^{*} \cup R\right)$, we assume that $L\left(\theta_{t} p, \varphi(t, p) x\right)=L(p, x)$ for some $t>0$ and $x \in(P \times X) \backslash\left(R^{*} \cup R\right)$. Then, $V^{*}\left(\theta_{-s} p, \varphi(-s, p) x\right)=V^{*}\left(\theta_{t-s} p, \varphi(t-s, p) x\right)$ for all $s \geq 0$. This is impossible, since $V^{*}(p, x) \in(0,1)$ and $\lim _{s \rightarrow \infty} V^{*}\left(\theta_{-s} p, \varphi(-s, p, x)\right)=0$.

In the following theorem, the above Lyapunov function for attractor-repeller pairs is extended to Morse decompositions.

\subsubsection{Theorem (Lyapunov Functions for Nonautonomous Morse Decompo-} SITIONS). Let $\left\{M_{1}, \ldots, M_{n}\right\}$ be a past (future, respectively) Morse decomposition. Then, there exists a function $L: P \times X \rightarrow[0,1]$ which is continuous with respect to $x \in X$ such that $\left.L\right|_{M_{i}} \equiv \frac{i-1}{n-1}$ for $i \in\{1, \ldots, n\}$ and 


$$
L\left(\theta_{t} p, \varphi(t, p) x\right)<L(p, x) \quad \text { for all } t>0 \text { and }(p, x) \in(P \times X) \backslash\left(M_{1} \cup \cdots \cup M_{n}\right)
$$

is satisfied.

Proof. Let $P \times X=R_{0} \supsetneq R_{1} \supsetneq \cdots \supsetneq R_{n}=\emptyset$ be the sequence of past repellers leading to the given past Morse decomposition, i.e.,

$$
M_{i}=R_{i}^{*} \cap R_{i-1} \quad \text { for all } i \in\{1, \ldots, n\} .
$$

Furthermore, let $L_{i}, i \in\{1, \ldots, n-1\}$, be the Lyapunov function corresponding to the past attractor-repeller pair $\left(R_{i}^{*}, R_{i}\right)$ as introduced in Lemma 3.3.1. We define

$$
L(p, x):=\frac{1}{n-1} \sum_{i=1}^{n-1} L_{i}(p, x) \text { for all }(p, x) \in P \times X .
$$

Choose $(p, x) \in M_{i}$ arbitrarily, and let $j \in\{1, \ldots, n-1\}$. Then $(p, x) \in R_{j}$ if and only if $j \in\{1, \ldots, i-1\}$, and $(p, x) \in R_{j}^{*}$ if and only if $j \in\{i, \ldots, n-1\}$. This implies $\left.L\right|_{M_{i}} \equiv \frac{i-1}{n-1}$ for all $i \in\{1, \ldots, n\}$. Now choose $(p, x) \in(P \times X) \backslash\left(M_{1} \cup \cdots \cup M_{n}\right)$. Then, there exists a $j \in\{1, \ldots, n\}$ with $(p, x) \notin R_{j}^{*} \cup R_{j}$. This means that $L_{j}\left(\theta_{t} p, \varphi(t, p) x\right)<L_{j}(p, x)$ for all $t>0$ and finishes the proof of this theorem.

\subsection{NonAutonomous Morse DeCOMPOSITIONS IN DIMENSION ONE}

In this section, Morse decompositions of nonautonomous dynamical systems whose phase space is a compact interval are studied. In this special case, stronger results concerning the convergence behavior of the system and the nonuniqueness of the Morse sets are obtained.

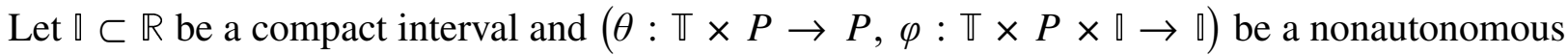
dynamical system.

\subsubsection{Theorem (Dynamical Properties of Nonautonomous Morse Decompo-} Sitions IN DimENSION ONE). Let $\left\{M_{1}, \ldots, M_{n}\right\}$ be a past Morse decomposition obtained by the finite sequence of past repellers $R_{0} \supset \cdots \supset R_{n}$. Then, the following statements are fulfilled:

(i) Pullback convergence. For all $p \in P$ and all functions $\gamma: \mathbb{T}^{+} \rightarrow \llbracket$ with

$$
\liminf _{t \rightarrow \infty} d\left(\gamma(t), \bigcup_{j=1}^{n} \partial R_{j}\left(\theta_{-t} p\right)\right)>0,
$$

we have

$$
\lim _{t \rightarrow \infty} d\left(\varphi\left(t, \theta_{-t} p\right) \gamma(t), \bigcup_{j=1}^{n} M_{j}(p)\right)=0 .
$$

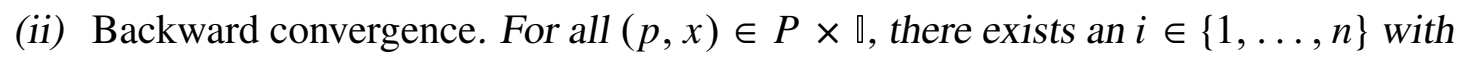




$$
\lim _{t \rightarrow \infty} d\left(\varphi(-t, p) x, M_{i}\left(\theta_{-t} p\right)\right)=0 .
$$

Let $\left\{M_{1}, \ldots, M_{n}\right\}$ be a future Morse decomposition obtained by the finite sequence of future attractors $A_{0} \subset \cdots \subset A_{n}$. Then, the following statements are fulfilled:

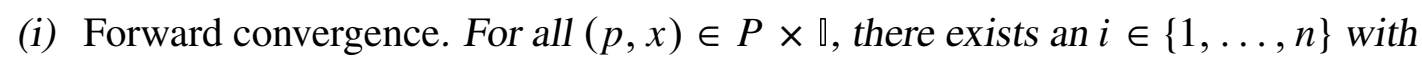

$$
\lim _{t \rightarrow \infty} d\left(\varphi(t, p) x, M_{i}\left(\theta_{t} p\right)\right)=0 .
$$

(ii) Pushforward convergence. For all $p \in P$ and all functions $\gamma: \mathbb{T}^{+} \rightarrow I$ with

$$
\liminf _{t \rightarrow \infty} d\left(\gamma(t), \bigcup_{j=1}^{n} \partial A_{j}\left(\theta_{t} p\right)\right)>0,
$$

we have

$$
\lim _{t \rightarrow \infty} d\left(\varphi\left(-t, \theta_{t} p\right) \gamma(t), \bigcup_{j=1}^{n} M_{j}(p)\right)=0
$$

PROOF. (i) This assertion is also valid for general Morse decompositions and was proved in Theorem 3.2.6.

(ii) Choose $(p, x) \in P \times \llbracket$ arbitrarily. Then, there exists an $i \in\{1, \ldots, n\}$ such that

$$
x \in R_{i}^{*}(p) \text { and } x \notin R_{i-1}^{*}(p) .
$$

In case $x \in R_{i-1}(p)$, the asserted limit relation follows, since then $x \in M_{i}(p)$ and $M_{i}$ is invariant. We therefore assume $x \notin R_{i-1}(p)$ from now on. Due to the topology of $\mathbb{1}, \varphi$ is order preserving in the following sense: For fixed $t \in \mathbb{T}$, exactly one of the following two statements is fulfilled:

- $y_{1}<y_{2}$ implies $\varphi(t, p) y_{1}<\varphi(t, p) y_{2}$,

- $y_{1}<y_{2}$ implies $\varphi(t, p) y_{1}>\varphi(t, p) y_{2}$.

Since $\lim _{t \rightarrow \infty} d\left(\varphi(-t, p) x, R_{i-1}\left(\theta_{-t} p\right)\right)=0$ (cf. Theorem 3.1 .5 (ii)), this implies that there exists a $y \in R_{i-1}(p)$ such that

$$
\lim _{t \rightarrow \infty}|\varphi(-t, p) x-\varphi(-t, p) y|=0 .
$$

Because $R_{i}^{*}$ is a past attractor, there exists an $\eta>0$ such that

$$
R_{i}^{*}(p)=\limsup _{t \rightarrow \infty} \varphi\left(t, \theta_{-t} p\right) U_{\eta}\left(R_{i}^{*}\left(\theta_{-t} p\right)\right)
$$

(cf. Remark 2.1 .3 (ii)). This implies $\lim \sup _{t \rightarrow \infty} \varphi\left(t, \theta_{-t} p\right) U_{\eta}(\varphi(-t, p) x) \subset R_{i}^{*}(p)$. Due to (3.5), this leads to $y \in R_{i}^{*}(p)$. Hence, $y \in M_{i}^{*}(p)$, and this finishes the proof of this theorem.

In our special situation, Proposition 3.1.6 can be generalized.

3.4.2 Proposition (Form of Nonuniqueness of The Morse Sets). Let $\left\{M_{1}, \ldots, M_{n}\right\}$ and $\left\{\hat{M}_{1}, \ldots, \hat{M}_{n}\right\}$ be past Morse decompositions obtained by the finite sequences of past repellers $R_{0} \supset \cdots \supset R_{n}$ and $\hat{R}_{0} \supset \cdots \supset \hat{R}_{n}$. We assume that 


$$
R_{i}^{*}=\hat{R}_{i}^{*} \quad \text { for all } i \in\{1, \ldots, n-1\}
$$

Then, the relation

$$
\lim _{t \rightarrow \infty} d_{H}\left(M_{i}\left(\theta_{-t} p\right), \hat{M}_{i}\left(\theta_{-t} p\right)\right)=0 \quad \text { for all } i \in\{1, \ldots, n\} \text { and } p \in P
$$

is fulfilled.

Let $\left\{M_{1}, \ldots, M_{n}\right\}$ and $\left\{\hat{M}_{1}, \ldots, \hat{M}_{n}\right\}$ be future Morse decompositions obtained by the finite sequences of future attractors $A_{0} \subset \cdots \subset A_{n}$ and $\hat{A}_{0} \subset \cdots \subset \hat{A}_{n}$. We assume that

$$
A_{i}^{*}=\hat{A}_{i}^{*} \quad \text { for all } i \in\{1, \ldots, n-1\} .
$$

Then, the relation

$$
\lim _{t \rightarrow \infty} d_{H}\left(M_{i}\left(\theta_{t} p\right), \hat{M}_{i}\left(\theta_{t} p\right)\right)=0 \quad \text { for all } i \in\{1, \ldots, n\} \text { and } p \in P
$$

is fulfilled.

Proof. Choose $i \in\{1, \ldots, n\}$ and $p \in P$ arbitrarily. W.l.o.g., we only show the relation

$$
\lim _{t \rightarrow \infty} d\left(M_{i}\left(\theta_{-t} p\right) \mid \hat{M}_{i}\left(\theta_{-t} p\right)\right)=0 .
$$

The proof is divided into three steps.

STEP 1 . There exists a past repeller $\bar{R}_{i-1} \supset R_{i-1}$ with $\bar{R}_{i-1}^{*}=R_{i-1}^{*}$ such that

$$
\bar{M}_{i}(\bar{p}):=R_{i}^{*}(\bar{p}) \cap \bar{R}_{i-1}(\bar{p}) \quad \text { for all } \bar{p} \in[p]
$$

has only finitely many connected components.

Since $R_{i}^{*}$ is a past attractor, there exists an $\eta>0$ such that

$$
R_{i}^{*}(p)=\limsup _{t \rightarrow \infty} \varphi\left(t, \theta_{-t} p\right) U_{\eta}\left(R_{i}^{*}\left(\theta_{-t} p\right)\right)
$$

(cf. Remark 2.1.3 (ii)). Since $\varphi$ is continuous and $U_{\eta}\left(R_{i}^{*}\left(\theta_{-t} p\right)\right.$ ) has only finitely many connected components for $t \in \mathbb{T}$, this implies that $R_{i}^{*}(p)$ has only finitely many connected components. Because $R_{i-1}$ is a past repeller, there exists a $\beta>0$ such that

$$
\lim _{t \rightarrow \infty} d\left(\varphi(-t, p) U_{\beta}\left(R_{i-1}(p)\right) \mid R_{i-1}\left(\theta_{-t} p\right)\right)=0 .
$$

Hence, the nonautonomous set $\bar{R}_{i-1}$, defined by

$$
\bar{R}_{i-1}(\bar{p}):=\left\{\begin{array}{cll}
\varphi(t, p) \operatorname{cls} U_{\frac{\beta}{2}}\left(R_{i-1}(p)\right) & : \bar{p}=\theta_{t} p \\
R_{i-1}(\bar{p}) & : \bar{p} \notin[p]
\end{array} \quad \text { for some } t \in \mathbb{T}\right.
$$

is also a past repeller fulfilling $\bar{R}_{i-1}^{*}=R_{i-1}^{*}$ (cf. Proposition 2.1.27 (iii)). Moreover, for all $\bar{p} \in[p]$, the set $\bar{R}_{i-1}(\bar{p})$ has only finitely many connected components, since $\varphi$ is continuous and cls $U_{\frac{\beta}{2}}\left(R_{i-1}(p)\right)$ has only finitely many connected components. This implies the assertion.

STEP 2. For all connected components $C$ of $\bar{M}_{i}(p)$, we have

$$
\lim _{t \rightarrow \infty} d\left(\varphi(-t, p) C \mid \hat{M}_{i}\left(\theta_{-t} p\right)\right)=0 .
$$


Let $C=\left[c_{1}, c_{2}\right]$ be a connected component of $\bar{M}_{i}(p)$, and choose $\varepsilon>0$ arbitrarily. Due to Theorem 3.4.1 (ii), there exists a $\tau_{1} \geq 0$ such that we have

$$
d\left(\varphi(-t, p) c_{j}, \hat{M}_{i}\left(\theta_{-t} p\right)\right) \leq \frac{\varepsilon}{2} \text { for all } t \geq \tau_{1} \text { and } j \in\{1,2\} .
$$

Furthermore, because of Proposition 3.1.6, there exists a $\tau_{2} \geq \tau_{1}$ with

$$
d_{H}\left(\bar{R}_{i-1}\left(\theta_{-t} p\right), \hat{R}_{i-1}\left(\theta_{-t} p\right)\right) \leq \frac{\varepsilon}{2} \quad \text { for all } t \geq \tau_{2} .
$$

Let $t \geq \tau_{2}$ and $x \in \varphi(-t, p) C$. In case $\min \left\{\left|x-\varphi(-t, p) c_{1}\right|,\left|x-\varphi(-t, p) c_{2}\right|\right\} \leq \frac{\varepsilon}{2}$, the inequality (3.6) implies that $d\left(x, \hat{M}_{i}\left(\theta_{-t} p\right)\right) \leq \varepsilon$. Otherwise, since $x \in \bar{R}_{i-1}\left(\theta_{-t} p\right)$ and due to (3.7), there exists a $y \in \hat{R}_{i-1}\left(\theta_{-t} p\right)$ with $|x-y| \leq \frac{\varepsilon}{2}$. Obviously,

$$
y \in \varphi(-t, p) C \subset \varphi(-t, p) \bar{M}_{i}(p) \subset R_{i}^{*}\left(\theta_{-t} p\right)
$$

is fulfilled. Hence, $y \in \hat{M}_{i}\left(\theta_{-t} p\right)$, and thus, $d\left(x, \hat{M}_{i}\left(\theta_{-t} p\right)\right) \leq \varepsilon$. This finishes the proof of this step.

STEP 3. The relation

$$
\lim _{t \rightarrow \infty} d\left(M_{i}\left(\theta_{-t} p\right) \mid \hat{M}_{i}\left(\theta_{-t} p\right)\right)=0
$$

is fulfilled.

Since $\bar{M}_{i}(p)$ has only finitely many connected components, this assertion follows from Step 2 and the fact that $\bar{M}_{i} \supset M_{i}$.

\subsection{Nonautonomous Morse Decompositions of Linear SYSTEMS}

In this section, Morse decompositions of linear nonautonomous dynamical systems are analyzed. Under the assumption that the base space is chain recurrent, such (autonomous) Morse decompositions of the corresponding skew product flow have been studied in SELGRADE [163], SALAMON \& ZEhnder [154] and Colonius \& KLIEMAnN [49, Chapter 5] (see also Colonius \& KliEMANN [48, 50] and BRAGA BARROS \& SAN MARTIN [34]).

Given $N \in \mathbb{N}$, let $\left(\theta: \mathbb{T} \times P \rightarrow P, \varphi: \mathbb{T} \times P \times \mathbb{R}^{N} \rightarrow \mathbb{R}^{N}\right)$ be a linear nonautonomous dynamical system, i.e.,

$$
\varphi(t, p, \alpha x+\beta y)=\alpha \varphi(t, p, x)+\beta \varphi(t, p, y) \quad \text { for all } \alpha, \beta \in \mathbb{R}, t \in \mathbb{T}, p \in P \text { and } x, y \in \mathbb{R}^{N} .
$$

Thus, there exists a matrix-valued function $\Phi: \mathbb{T} \times P \rightarrow \mathbb{R}^{N \times N}$ with $\Phi(t, p) x=\varphi(t, p, x)$ for all $t \in \mathbb{T}, p \in P$ and $x \in \mathbb{R}^{N}$. We suppose that $(\theta, \varphi)$ is invertible, i.e., $\mathbb{T}=\mathbb{R}$ or $\mathbb{T}=\mathbb{Z}$.

For our purpose, $\mathbb{R}^{N}$ is equipped with the Euclidian norm $\|\cdot\|$, induced by the Euclidian scalar product (see Section 1.1). The NDS $(\theta, \varphi)$ canonically induces a nonautonomous dynamical system $(\theta, \mathbb{P} \Phi)$ on the real projective space $\mathbb{P}^{N-1}$ of the vector space $\mathbb{R}^{N}$ by defining

$$
\mathbb{P} \Phi(t, p) \mathbb{P} x:=\mathbb{P}(\Phi(t, p) x) \text { for all } t \in \mathbb{T}, p \in P \text { and } x \in \mathbb{R}^{N}
$$

(see also Colonius \& KLIEMAnN [49, Lemma 5.2.1, p. 149]). For basic properties of the projective space and notation, we refer to Appendix A.3. 
The main observation of the following lemma is that past attractors and future repellers in $\mathbb{P}^{N-1}$ are linear nonautonomous invariant manifolds in $\mathbb{R}^{N}$ (cf. Definition 4.1.1). For a similar result, see Salamon \& Zehnder [154, Proposition 2.9] and Colonius \& Kliemann [49, Lemma 5.2.2., p. 149].

\subsubsection{Proposition (PAst Attractors and Future Repellers in $\mathbb{P}^{N-1}$ ). Let $A$ be} a past attractor of $(\theta, \mathbb{P} \Phi)$. Then, for all $p \in P$ and all compact sets $C \subset \mathbb{S}^{N-1} \backslash \mathbb{P}^{-1} A(p)$, we have

$$
\lim _{t \rightarrow \infty} \frac{\sup _{v \in \mathbb{S}^{N-1} \cap \mathbb{P}^{-1} A(p)}\|\Phi(-t, p) v\|}{\inf _{w \in C}\|\Phi(-t, p) w\|}=0 .
$$

Moreover, $\mathbb{P}^{-1} A$ is a linear nonautonomous invariant manifold in $\mathbb{R}^{N}$, i.e., $\mathbb{P}^{-1} A$ is an invariant nonautonomous set and for all $p \in P$, the set $\mathbb{P}^{-1} A(p)$ is a linear subspace of the $\mathbb{R}^{N}$. Let $R$ be a future repeller of $(\theta, \mathbb{P} \Phi)$. Then, for all $p \in P$ and all compact sets $C \subset \mathbb{S}^{N-1} \backslash \mathbb{P}^{-1} R(p)$, we have

$$
\lim _{t \rightarrow \infty} \frac{\sup _{v \in \mathbb{S}^{N-1} \cap \mathbb{P}^{-1} R(p)}\|\Phi(t, p) v\|}{\inf _{w \in C}\|\Phi(t, p) w\|}=0 .
$$

Moreover, $\mathbb{P}^{-1} R$ is a linear nonautonomous invariant manifold in $\mathbb{R}^{N}$.

Proof. Let $A$ be a past attractor of $(\theta, \mathbb{P} \Phi)$, and choose a $p \in P$ and a compact set $C \subset \mathbb{S}^{N-1} \backslash \mathbb{P}^{-1} A(p)$ arbitrarily. First, we define for any nonzero $v \in \mathbb{P}^{-1} A(p)$ and $w \in C$ the two-dimensional linear subspace $L_{v, w} \subset \mathbb{R}^{N}$ by

$$
L_{v, w}:=\{r v+s w: r, s \in \mathbb{R}\} .
$$

The proof of this proposition is divided into five steps.

STEP 1. For all $0 \neq v \in \mathbb{P}^{-1} A(p)$ and $w \in C$ such that $\mathbb{P} v$ is a boundary point of $A(p) \cap \mathbb{P} L_{v, w}$ relative to $\mathbb{P} L_{v, w}$, we have

$$
\lim _{t \rightarrow \infty} \frac{\|\Phi(-t, p) v\|}{\|\Phi(-t, p) w\|}=0
$$

Since $A$ is a past attractor, there exists an $\eta>0$ such that

$$
\lim _{t \rightarrow \infty} d\left(\mathbb{P} \Phi\left(t, \theta_{-t} p\right) U_{2 \eta}\left(A\left(\theta_{-t} p\right)\right) \mid A(p)\right)=0 \text {. }
$$

Due to Lemma A.3.1, there exists a $\delta \in(0,1)$ such that for all nonzero $u_{1}, u_{2} \in \mathbb{R}^{N}$ with

$$
\frac{\left\langle u_{1}, u_{2}\right\rangle^{2}}{\left\|u_{1}\right\|^{2}\left\|u_{2}\right\|^{2}} \geq 1-\delta
$$

we have

$$
d_{\mathbb{P}}\left(\mathbb{P} u_{1}, \mathbb{P} u_{2}\right) \leq \eta
$$

We argue negatively and suppose that there exist a $\gamma>0$ and a sequence $\left\{t_{n}\right\}_{n \in \mathbb{N}}$ with $\lim _{n \rightarrow \infty} t_{n}=-\infty$ such that

$$
\frac{\left\|\Phi\left(t_{n}, p\right) w\right\|}{\left\|\Phi\left(t_{n}, p\right) v\right\|} \leq \gamma \quad \text { for all } n \in \mathbb{N}
$$


For nonzero $c \in \mathbb{R}$ with $|c|$ sufficiently small, this implies that for all $n \in \mathbb{N}$,

$$
\begin{aligned}
& \frac{\left\langle\Phi\left(t_{n}, p\right)(c w+v), \Phi\left(t_{n}, p\right) v\right\rangle^{2}}{\left\|\Phi\left(t_{n}, p\right)(c w+v)\right\|^{2}\left\|\Phi\left(t_{n}, p\right) v\right\|^{2}} \\
= & \frac{c^{2}\left\langle\Phi\left(t_{n}, p\right) w, \Phi\left(t_{n}, p\right) v\right\rangle^{2}+2 c\left\|\Phi\left(t_{n}, p\right) v\right\|^{2}\left\langle\Phi\left(t_{n}, p\right) w, \Phi\left(t_{n}, p\right) v\right\rangle+\left\|\Phi\left(t_{n}, p\right) v\right\|^{4}}{c^{2}\left\|\Phi\left(t_{n}, p\right) w\right\|^{2}\left\|\Phi\left(t_{n}, p\right) v\right\|^{2}+2 c\left\|\Phi\left(t_{n}, p\right) v\right\|^{2}\left\langle\Phi\left(t_{n}, p\right) w, \Phi\left(t_{n}, p\right) v\right\rangle+\left\|\Phi\left(t_{n}, p\right) v\right\|^{4}} \\
\geq & 1-\delta
\end{aligned}
$$

holds. Hence, for $|c|>0$ sufficiently small, we have

$$
d_{\mathbb{P}}\left(\mathbb{P} \Phi\left(t_{n}, p\right) \mathbb{P}(c w+v), A\left(\theta_{t_{n}} p\right)\right) \leq \eta \quad \text { for all } n \in \mathbb{N} .
$$

This implies

$$
\begin{aligned}
d_{\mathbb{P}}(\mathbb{P}(c w+v), A(p)) & =\lim _{n \rightarrow \infty} d_{\mathbb{P}}(\mathbb{P}(c w+v), A(p)) \\
& =\lim _{n \rightarrow \infty} d_{\mathbb{P}}(\mathbb{P} \Phi\left(-t_{n}, \theta_{t_{n}} p\right) \underbrace{\mathbb{P} \Phi\left(t_{n}, p\right) \mathbb{P}(c w+v)}_{\in U_{2 \eta}\left(A\left(\theta_{t_{n}} p\right)\right)}, A(p)) \\
& \stackrel{(3.8)}{=} 0 .
\end{aligned}
$$

This is a contradiction, since $\mathbb{P} v$ is assumed to be a boundary point of $A(p) \cap \mathbb{P} L_{v, w}$ in $\mathbb{P} L_{v, w}$, and thus, the first step of this proof is finished.

STEP 2. For all nonzero $v \in \mathbb{P}^{-1} A(p)$ and $w \in C$, the set $A(p) \cap \mathbb{P} L_{v, w}$ is a singleton.

Please note that any point in $\mathbb{P} L_{v, w} \backslash\{\mathbb{P} v\}$ is given by $\mathbb{P}(w+c v)$ for some $c \in \mathbb{R}$. It follows from Step 1 that

$$
\begin{aligned}
& \lim _{t \rightarrow-\infty} \frac{\langle\Phi(t, p)(w+c v), \Phi(t, p) w\rangle^{2}}{\|\Phi(t, p)(w+c v)\|^{2}\|\Phi(t, p) w\|^{2}} \\
= & \lim _{t \rightarrow-\infty} \frac{\|\Phi(t, p) w\|^{4}+2 c\|\Phi(t, p) w\|^{2}\langle\Phi(t, p) v, \Phi(t, p) w\rangle+c^{2}\langle\Phi(t, p) v, \Phi(t, p) w\rangle^{2}}{\|\Phi(t, p) w\|^{4}+2 c\|\Phi(t, p) w\|^{2}\langle\Phi(t, p) v, \Phi(t, p) w\rangle+c^{2}\|\Phi(t, p) v\|^{2}\|\Phi(t, p) w\|^{2}} \\
= & 1
\end{aligned}
$$

in case $\mathbb{P} v$ is a boundary point of $A(p) \cap \mathbb{P} L_{v, w}$ relative to $\mathbb{P} L_{v, w}$. This implies with Lemma A.3.1 that

$$
\lim _{t \rightarrow \infty} d_{\mathbb{P}}(\mathbb{P} \Phi(-t, p) \mathbb{P}(w+c v), \mathbb{P} \Phi(-t, p) \mathbb{P} w)=0,
$$

and hence, $\mathbb{P}(w+c v) \notin A(p)$. Therefore, $A(p) \cap \mathbb{P} L_{v, w}$ consists of a single point.

STEP 3. For all nonzero $v \in \mathbb{P}^{-1} A(p)$ and $w \in C$, we have

$$
\lim _{t \rightarrow \infty} \frac{\|\Phi(-t, p) v\|}{\|\Phi(-t, p) w\|}=0
$$

This follows directly from Step 1 and Step 2.

STEP 4. $\mathbb{P}^{-1} A(p)$ is a linear subspace of $\mathbb{R}^{N}$.

We have shown that for any two-dimensional subspace $L_{v, w}$, the set $A(p) \cap \mathbb{P} L_{v, w}$ is either empty, equals $\mathbb{P} L_{v, w}$ or consists of a single point. This implies that $\mathbb{P}^{-1} A$ intersects each fiber in a linear subspace.

STEP 5. We have

$$
\lim _{t \rightarrow \infty} \frac{\sup _{v \in \mathbb{S}^{N-1} \cap \mathbb{P}^{-1} A(p)}\|\Phi(-t, p) v\|}{\inf _{w \in C}\|\Phi(-t, p) w\|}=0
$$


We assume to the contrary that there exist sequences $\left\{t_{n}\right\}_{n \in \mathbb{N}}$ in $\mathbb{R},\left\{v_{n}\right\}_{n \in \mathbb{N}}$ in $\mathbb{S}^{N-1} \cap \mathbb{P}^{-1} A(p)$ and $\left\{w_{n}\right\}_{n \in \mathbb{N}}$ in $C$ such that $\lim _{n \rightarrow \infty} t_{n}=-\infty$ and, w.l.o.g., $\lim _{n \rightarrow \infty} v_{n}=v$ and $\lim _{n \rightarrow \infty} w_{n}=w$ for some $v \in \mathbb{P}^{-1} A(p) \cap \mathbb{S}^{N-1}$ and $w \in C$, and the following property is fulfilled: There exists a $\gamma>0$ such that

$$
\frac{\left\|\Phi\left(t_{n}, p\right) w_{n}\right\|}{\left\|\Phi\left(t_{n}, p\right) v_{n}\right\|} \leq \gamma \quad \text { for all } n \in \mathbb{N} .
$$

We write $\Phi_{n}:=\Phi\left(t_{n}, p\right)$. Similarly to Step 1 , for nonzero $c \in \mathbb{R}$ with $|c|$ sufficiently small, this implies that for all $n \in \mathbb{N}$,

$$
\begin{aligned}
& \frac{\left\langle\Phi_{n}\left(c w_{n}+v_{n}\right), \Phi_{n} v_{n}\right\rangle^{2}}{\left\|\Phi_{n}\left(c w_{n}+v_{n}\right)\right\|^{2}\left\|\Phi_{n} v_{n}\right\|^{2}} \\
= & \frac{c^{2}\left\langle\Phi_{n} w_{n}, \Phi_{n} v_{n}\right\rangle^{2}+2 c\left\|\Phi_{n} v_{n}\right\|^{2}\left\langle\Phi_{n} w_{n}, \Phi_{n} v_{n}\right\rangle+\left\|\Phi_{n} v_{n}\right\|^{4}}{c^{2}\left\|\Phi_{n} w_{n}\right\|^{2}\left\|\Phi_{n} v_{n}\right\|^{2}+2 c\left\|\Phi_{n} v_{n}\right\|^{2}\left\langle\Phi_{n} w_{n}, \Phi_{n} v_{n}\right\rangle+\left\|\Phi_{n} v_{n}\right\|^{4}} \\
\geq & 1-\delta
\end{aligned}
$$

holds, with $\delta \in(0,1)$ chosen as in Step 1 . Hence, for $|c|>0$ sufficiently small, we have

$$
d_{\mathbb{P}}\left(\mathbb{P} \Phi\left(t_{n}, p\right) \mathbb{P}\left(c w_{n}+v_{n}\right), A\left(\theta_{t_{n}} p\right)\right) \leq \eta \quad \text { for all } n \in \mathbb{N}
$$

Since $\mathbb{P}(c w+v) \notin A(p)$ (due to Step 2, $A(p) \cap \mathbb{P} L_{v, w}$ is a singleton), there exist an $n_{0} \in \mathbb{N}$ and a $\beta>0$ such that $c w_{n}+v_{n} \notin U_{\beta}(A(p))$ for all $n \geq n_{0}$. Similarly to Step 1, using (3.8), this implies a contradiction.

Concerning past repellers and future attractors, we can not expect that their fibers give rise to linear subspaces, since they are intrinsically nonunique (cf. Proposition 2.1.27). The following lemma, however, says that for any past attractor or future repeller, a linear counterpart in form of a past repeller or future attractor, respectively, can be found easily.

3.5.2 Proposition (PAst Repellers and Future Attractors in $\mathbb{P}^{N-1}$ ). Let $A$ be a past attractor of $(\theta, \mathbb{P} \Phi)$ and $R \subset P \times \mathbb{P}^{N-1}$ be an invariant nonautonomous set such that $\mathbb{P}^{-1} R(p)$ is a linear subspace of the $\mathbb{R}^{N}$ and

$$
\mathbb{P}^{-1} A(p) \oplus \mathbb{P}^{-1} R(p)=\mathbb{R}^{N} \text { for all } p \in P .
$$

Then, $R$ is a past repeller, and the relation $A=R^{*}$ is fulfilled.

Let $R$ be a future repeller of $(\theta, \mathbb{P} \Phi)$ and $A \subset P \times \mathbb{P}^{N-1}$ be an invariant nonautonomous set such that $\mathbb{P}^{-1} A(p)$ is linear subspace of the $\mathbb{R}^{N}$ and

$$
\mathbb{P}^{-1} A(p) \oplus \mathbb{P}^{-1} R(p)=\mathbb{R}^{N} \text { for all } p \in P \text {. }
$$

Then, $A$ is a future attractor, and the relation $R=A^{*}$ is fulfilled.

PROOF. The proof of this proposition is divided into five steps.

STEP 1. For all $p \in P$ and compact sets $C \subset \mathbb{P}^{N-1}$ with $C \cap A(p)=\emptyset$, we have

$$
\lim _{t \rightarrow \infty} \inf _{0 \neq v \in \mathbb{P}^{-1} C} \frac{\left\|\Phi(-t, p) v_{r}\right\|}{\|\Phi(-t, p) v\|}=\lim _{t \rightarrow \infty} \sup _{0 \neq v \in \mathbb{P}^{-1} C} \frac{\left\|\Phi(-t, p) v_{r}\right\|}{\|\Phi(-t, p) v\|}=1,
$$


where $v=v_{a}+v_{r}$ with $v_{a} \in \mathbb{P}^{-1} A(p)$ and $v_{r} \in \mathbb{P}^{-1} R(p)$.

The first assertion follows from

$$
\begin{aligned}
\lim _{t \rightarrow \infty} \inf _{0 \neq v \in \mathbb{P}^{-1} C} \frac{\left\|\Phi(-t, p) v_{r}\right\|}{\|\Phi(-t, p) v\|} & \geq\left(\lim _{t \rightarrow \infty} \sup _{0 \neq v \in \mathbb{P}^{-1} C} \frac{\left\|\Phi(-t, p) v_{a}\right\|}{\left\|\Phi(-t, p) v_{r}\right\|}+1\right)^{-1} \\
& =\left(\lim _{t \rightarrow \infty} \sup _{v \in \mathbb{P}^{-1} C, v_{a} \neq 0} \frac{\left\|v_{a}\right\|\left\|\Phi(-t, p) \frac{v_{a}}{\left\|v_{a}\right\|}\right\|}{\left\|v_{r}\right\|\left\|\Phi(-t, p) \frac{v_{r}}{\left\|v_{r}\right\|}\right\|}+1\right)^{-1} \\
& \stackrel{\text { Prop. 3.5.1 }}{=} 1
\end{aligned}
$$

and

$$
\begin{aligned}
\lim _{t \rightarrow \infty} \inf _{0 \neq v \in \mathbb{P}^{-1} C} \frac{\left\|\Phi(-t, p) v_{r}\right\|}{\|\Phi(-t, p) v\|} & \leq\left(\lim _{t \rightarrow \infty} \sup _{0 \neq v \in \mathbb{P}^{-1} C}\left|1-\frac{\left\|\Phi(-t, p) v_{a}\right\|}{\left\|\Phi(-t, p) v_{r}\right\|}\right|\right)^{-1} \\
& =\left(\lim _{t \rightarrow \infty} \sup _{v \in \mathbb{P}^{-1} C, v_{a} \neq 0}\left|1-\frac{\left\|v_{a}\right\|\left\|\Phi(-t, p) \frac{v_{a}}{\left\|v_{a}\right\|}\right\|}{\left\|v_{r}\right\|\left\|\Phi(-t, p) \frac{v_{r}}{\left\|v_{r}\right\|}\right\|}\right|\right)^{-1}
\end{aligned}
$$$$
\stackrel{\text { Prop. 3.5.1 }}{=} 1 \text {. }
$$

In both relations, Proposition 3.5.1 is applicable, because the set $\left\{v_{a}: v \in \mathbb{P}^{-1} C \cap \mathbb{S}^{N-1}\right\}$ is compact and the set $\left\{v_{r}: v \in \mathbb{P}^{-1} C \cap \mathbb{S}^{N-1}\right\}$ is bounded away from zero. This is due to the fact that the projector $Q \in \mathbb{R}^{N \times N}$ with range $A(p)$ and null space $R(p)$ satisfies

$$
\begin{aligned}
& \left\{v_{a}: v \in \mathbb{P}^{-1} C \cap \mathbb{S}^{N-1}\right\}=Q\left(\mathbb{P}^{-1} C \cap \mathbb{S}^{N-1}\right) \\
& \left\{v_{r}: v \in \mathbb{P}^{-1} C \cap \mathbb{S}^{N-1}\right\}=(\mathbb{1}-Q)\left(\mathbb{P}^{-1} C \cap \mathbb{S}^{N-1}\right)
\end{aligned} \quad \text { and }
$$

(cf. also Step 3 of the proof of Lemma 4.1.14). The assertion

$$
\lim _{t \rightarrow \infty} \sup _{0 \neq v \in \mathbb{P}^{-1} C} \frac{\left\|\Phi(-t, p) v_{r}\right\|}{\|\Phi(-t, p) v\|}=1
$$

follows analogously.

STEP 2. For all $p \in P$ and compact sets $C \subset \mathbb{P}^{N-1}$ with $C \cap A(p)=\emptyset$, we have

$$
\lim _{t \rightarrow \infty} d_{\mathbb{P}}\left(\mathbb{P} \Phi(-t, p) C \mid R\left(\theta_{-t} p\right)\right)=0 .
$$

With $v_{a}$ and $v_{r}$ defined as in Step 1 , for all $t \geq 0$ and $v \in \mathbb{S}^{N-1} \cap \mathbb{P}^{-1} C$, we consider the expression

$$
\begin{aligned}
& \frac{\left\langle\Phi(-t, p) v, \Phi(-t, p) v_{r}\right\rangle^{2}}{\|\Phi(-t, p) v\|^{2}\left\|\Phi(-t, p) v_{r}\right\|^{2}}=\frac{\left(\left\langle\Phi(-t, p) v_{a}, \Phi(-t, p) v_{r}\right\rangle+\left\langle\Phi(-t, p) v_{r}, \Phi(-t, p) v_{r}\right\rangle\right)^{2}}{\|\Phi(-t, p) v\|^{2}\left\|\Phi(-t, p) v_{r}\right\|^{2}} \\
= & \frac{\left\langle\Phi(-t, p) v_{a}, \Phi(-t, p) v_{r}\right\rangle^{2}+\left\|\Phi(-t, p) v_{r}\right\|^{4}+2\left\langle\Phi(-t, p) v_{a}, \Phi(-t, p) v_{r}\right\rangle\left\|\Phi(-t, p) v_{r}\right\|^{2}}{\|\Phi(-t, p) v\|^{2}\left\|\Phi(-t, p) v_{r}\right\|^{2}} \\
= & \frac{\left\langle\Phi(-t, p) v_{a}, \Phi(-t, p) v_{r}\right\rangle^{2}}{\|\Phi(-t, p) v\|^{2}\left\|\Phi(-t, p) v_{r}\right\|^{2}}+\frac{\left\|\Phi(-t, p) v_{r}\right\|^{2}}{\|\Phi(-t, p) v\|^{2}}+\frac{2\left\langle\Phi(-t, p) v_{a}, \Phi(-t, p) v_{r}\right\rangle}{\|\Phi(-t, p) v\|^{2}} .
\end{aligned}
$$


Using the Cauchy-Schwartz inequality, we obtain the following relations:

$$
\begin{aligned}
& 0 \leq \lim _{t \rightarrow \infty} \sup _{v \in \mathbb{S}^{N-1} \cap \mathbb{P}^{-1} C} \frac{\left\langle\Phi(-t, p) v_{a}, \Phi(-t, p) v_{r}\right\rangle^{2}}{\|\Phi(-t, p) v\|^{2}\left\|\Phi(-t, p) v_{r}\right\|^{2}} \leq \\
& \lim _{t \rightarrow \infty} \sup _{v \in \mathbb{S}^{N-1} \cap \mathbb{P}^{-1} C} \frac{\left\|\Phi(-t, p) v_{a}\right\|^{2}}{\|\Phi(-t, p) v\|^{2}} \stackrel{\text { Proposition 3.5.1 }}{=} 0
\end{aligned}
$$

and

$$
\begin{aligned}
0 & \leq \quad \lim _{t \rightarrow \infty} \sup _{v \in \mathbb{S}^{N-1} \cap \mathbb{P}^{-1} C} \frac{2\left|\left\langle\Phi(-t, p) v_{a}, \Phi(-t, p) v_{r}\right\rangle\right|}{\|\Phi(-t, p) v\|^{2}} \\
& \leq \lim _{t \rightarrow \infty} \sup _{v \in \mathbb{S}^{N-1} \cap \mathbb{P}^{-1} C} 2 \frac{\left\|\Phi(-t, p) v_{a}\right\|}{\left\|\Phi(-t, p) v_{r}\right\|} \\
\stackrel{\text { Step } 1}{=} & \lim _{t \rightarrow \infty} \sup _{v \in \mathbb{S}^{N-1} \cap \mathbb{P}^{-1} C} \frac{2\left\|\Phi(-t, p) v_{a}\right\|}{\|\Phi(-t, p) v\|} \\
\stackrel{\|(-t, p) v\|}{=} &
\end{aligned}
$$

Hence, we obtain

$$
\begin{aligned}
& \lim _{t \rightarrow \infty} \inf _{v \in \mathbb{S}^{N-1} \cap \mathbb{P}^{-1} C} \frac{\left\langle\Phi(-t, p) v, \Phi(-t, p) v_{r}\right\rangle^{2}}{\|\Phi(-t, p) v\|^{2}\left\|\Phi(-t, p) v_{r}\right\|^{2}} \\
&=\lim _{t \rightarrow \infty} \inf _{v \in \mathbb{S}^{N-1} \cap \mathbb{P}^{-1} C}\left(\frac{\left\langle\Phi(-t, p) v_{a}, \Phi(-t, p) v_{r}\right\rangle^{2}}{\|\Phi(-t, p) v\|^{2}\left\|\Phi(-t, p) v_{r}\right\|^{2}}+\frac{\left\|\Phi(-t, p) v_{r}\right\|^{2}}{\|\Phi(-t, p) v\|^{2}}\right. \\
&\left.+\frac{2\left\langle\Phi(-t, p) v_{a}, \Phi(-t, p) v_{r}\right\rangle}{\|\Phi(-t, p) v\|^{2}}\right) \\
& \stackrel{+ \text { Step } 1 .}{=} 1 .
\end{aligned}
$$

Using Lemma A.3.1, this implies the assertion.

STEP 3. $A$ and $R$ are past isolated, i.e., there exists a $\beta>0$ such that for all $p \in P$, there exists a $\tau>0$ with

$$
U_{\beta}\left(A\left(\theta_{-t} p\right)\right) \cap U_{\beta}\left(R\left(\theta_{-t} p\right)\right)=\emptyset \quad \text { for all } t \geq \tau .
$$

Since $A$ is a past attractor, Proposition 2.1.2 (iii) implies that there exists an $\eta>0$ such that for all $p \in P$, we have

$$
\lim _{t \rightarrow \infty} d_{\mathbb{P}}\left(\varphi\left(t, \theta_{-t} p\right) U_{\eta}\left(A\left(\theta_{-t} p\right)\right) \mid A(p)\right)=0 .
$$

Defining $\beta:=\frac{\eta}{2}$ and using the invariance of $R$, this implies the assertion.

STEP $4 . R$ is a past repeller.

This is a direct consequence of Step 2 and Step 3.

STEP 5. The relation $A=R^{*}$ is fulfilled.

We define $\eta>0, P^{*}$ and $B_{\zeta}$ for $\zeta \in(0, \eta]$ as in the introduction of Section 3.1. We also consider the collection $\mathcal{M}:=\left\{B_{\zeta}: \zeta \in(0, \eta]\right\}$. Due to Theorem 3.1.1, it is sufficient to show that $A$ is an $\mathcal{M}$-past attractor. Thereto, we fix $\zeta \in(0, \eta]$ and $p \in P^{*}$. Furthermore, we choose $\varepsilon>0$ arbitrarily and consider the compact set $C:=\mathbb{P}^{N-1} \backslash U_{\varepsilon}(A(p))$. Due to Step 2, we have

$$
\lim _{t \rightarrow \infty} d\left(\mathbb{P} \Phi(-t, p) C \mid R\left(\theta_{-t} p\right)\right)=0 .
$$


This implies that there exists a $\tau>0$ such that $\mathbb{P} \Phi(-t, p) C \cap B_{\zeta}\left(\theta_{-t} p\right)=\emptyset$ for all $t \geq \tau$. Thus,

$$
d_{\mathbb{P}}\left(\Phi\left(t, \theta_{-t} p\right) B_{\zeta}\left(\theta_{-t} p\right) \mid A(p)\right) \leq \varepsilon \quad \text { for all } t \geq \tau .
$$

Due to Proposition 2.1.2 (i), $A$ is an $\mathcal{M}$-past attractor. This finishes the proof of this proposition.

3.5.3 LEMMA. For all $n \in \mathbb{N}$, we consider nontrivial linear subspaces $W_{n} \subsetneq V_{n} \subsetneq \mathbb{R}^{N}$. Furthermore, let $\left\{x_{n}\right\}_{n \in \mathbb{N}}$ be a sequence in $\mathbb{R}^{N}$ such that the following hypotheses are fulfilled:

(i) $x_{n} \notin V_{n}$ for all $n \in \mathbb{N}$,

(ii) $\lim _{n \rightarrow \infty} d_{\mathbb{P}}\left(\mathbb{P} x_{n}, \mathbb{P} V_{n}\right)=0$,

(iii) there exists an $\varepsilon>0$ such that $d_{\mathbb{P}}\left(\mathbb{P} x_{n}, \mathbb{P} W_{n}\right) \geq \varepsilon$ for all $n \in \mathbb{N}$.

For all $n \in \mathbb{N}$, we define $C_{n}:=W_{n} \oplus\left\{\lambda x_{n}: \lambda \in \mathbb{R}\right\}$. Then, the limit relation

$$
\lim _{n \rightarrow \infty} d_{\mathbb{P}}\left(\mathbb{P} C_{n} \mid \mathbb{P} V_{n}\right)=0
$$

is fulfilled.

Proof. W.1.o.g., we assume that $\left\|x_{n}\right\|=1$ for all $n \in \mathbb{N}$. Due to Hypothesis (ii), there exists a sequence $\left\{v_{n}\right\}_{n \in \mathbb{N}}$ with $v_{n} \in V_{n}$ and $\left\|v_{n}\right\|=1$ for all $n \in \mathbb{N}$ such that $\lim _{n \rightarrow \infty}\left\|x_{n}-v_{n}\right\|=0$. Since $\mathbb{P} C_{n}$ is a compact subset of $\mathbb{P}^{N-1}$, there exists a sequence $\left\{c_{n}\right\}_{n \in \mathbb{N}}$ with $c_{n} \in C_{n}$ for all $n \in \mathbb{N}$ such that $d_{\mathbb{P}}\left(\mathbb{P} C_{n} \mid \mathbb{P} V_{n}\right)=d_{\mathbb{P}}\left(\mathbb{P} c_{n}, \mathbb{P} V_{n}\right)$. W.l.o.g., we assume that $c_{n}$ is of the form

$$
c_{n}=x_{n}+w_{n} \quad \text { for all } n \in \mathbb{N} \text {, }
$$

where $\left\{w_{n}\right\}_{n \in \mathbb{N}}$ is a sequence with $w_{n} \in W_{n}$ for all $n \in \mathbb{N}$, and we define

$$
r_{n}:=v_{n}+w_{n} \quad \text { for all } n \in \mathbb{N}
$$

and $\beta_{n}:=\left\langle x_{n}, w_{n}\right\rangle, \delta_{n}:=\left\langle v_{n}, w_{n}\right\rangle$ and $\gamma_{n}:=\left\langle x_{n}, v_{n}\right\rangle$ for all $n \in \mathbb{N}$. Then, for all $n \in \mathbb{N}$, we have

$$
\frac{\left\langle c_{n}, r_{n}\right\rangle^{2}}{\left\|c_{n}\right\|^{2}\left\|r_{n}\right\|^{2}}=\frac{\gamma_{n}^{2}+\overbrace{\beta_{n}^{2}+\delta_{n}^{2}+\left\|w_{n}\right\|^{4}+2 \gamma_{n}\left\|x_{n}\right\|^{2}+2 \beta_{n} \delta_{n}}^{=: \xi_{2\left(\beta_{n}+\delta_{n}\right)\left(\left\|w_{n}\right\|^{2}+\gamma_{n}\right)}}}{1+\underbrace{2\left\|w_{n}\right\|^{2}+\left\|w_{n}\right\|^{4}+4 \beta_{n} \delta_{n}}_{=: \bar{\xi}_{n}}+\underbrace{2\left(\beta_{n}+\delta_{n}\right)\left(\left\|w_{n}\right\|^{2}+1\right)}_{=: \bar{\eta}_{n}}},
$$

and it is easy to see that $\lim _{n \rightarrow \infty} \gamma_{n}=1, \lim _{n \rightarrow \infty} \xi_{n} / \bar{\xi}_{n}=1$ and $\lim _{n \rightarrow \infty} \eta_{n} / \bar{\eta}_{n}=1$. This implies that

$$
\lim _{n \rightarrow \infty} \frac{\left\langle c_{n}, r_{n}\right\rangle^{2}}{\left\|c_{n}\right\|^{2}\left\|r_{n}\right\|^{2}}=1
$$

under the condition that $\left\|c_{n}\right\|\left\|r_{n}\right\|$ is bounded away from 0 in the limit $n \rightarrow \infty$. To see that this is fulfilled, we need Hypothesis (iii), which says that there exists a $\delta \in(0,1)$ with

$$
\frac{\left\langle x_{n}, w\right\rangle}{\|w\|} \leq \delta \quad \text { for all } n \in \mathbb{N} \text { and } w \in W_{n}
$$


(cf. Lemma A.3.1). This means that for all $n \in \mathbb{N}$ and $w \in W_{n}$, we have

$$
\left\|x_{n}-w\right\|^{2}=1-2\left\langle x_{n}, w\right\rangle+\|w\|^{2} \geq 1-2 \delta\|w\|+\|w\|^{2} \geq \gamma \quad \text { for some } \gamma>0,
$$

and using $\lim _{n \rightarrow \infty}\left\|x_{n}-v_{n}\right\|=0$, this finishes the proof of this lemma.

In our special situation, convergence in both directions to the Morse sets is satisfied.

\subsubsection{Theorem (Dynamical Properties of Nonautonomous Morse Decompo-} SITIONS OF LINEAR SYSTEMS). Let $\left\{M_{1}, \ldots, M_{n}\right\}$ be a past Morse decomposition obtained by the finite sequence of past repellers $R_{0} \supset \cdots \supset R_{n}$ such that $\mathbb{P}^{-1} R_{i}(p)$ is a linear subspace of $\mathbb{R}^{N}$ for $i \in\{1, \ldots, n-1\}$ and $p \in P$. Then, the following statements are fulfilled:

(i) Pullback convergence. For all $p \in P$ and all functions $\gamma: \mathbb{T}^{+} \rightarrow \mathbb{P}^{N-1}$ with

$$
\liminf _{t \rightarrow \infty} d_{\mathbb{P}}\left(\gamma(t), \bigcup_{j=1}^{n} \partial R_{j}\left(\theta_{-t} p\right)\right)>0
$$

we have

$$
\lim _{t \rightarrow \infty} d_{\mathbb{P}}\left(\mathbb{P} \Phi\left(t, \theta_{-t} p\right) \gamma(t), \bigcup_{j=1}^{n} M_{j}(p)\right)=0 .
$$

(ii) Backward convergence. For all $(p, x) \in P \times \mathbb{P}^{N-1}$, there exists an $i \in\{1, \ldots, n\}$ with

$$
\lim _{t \rightarrow \infty} d_{\mathbb{P}}\left(\mathbb{P} \Phi(-t, p) x, M_{i}\left(\theta_{-t} p\right)\right)=0 .
$$

Let $\left\{M_{1}, \ldots, M_{n}\right\}$ be a future Morse decomposition obtained by the finite sequence of future attractors $A_{0} \subset \cdots \subset A_{n}$ such that $\mathbb{P}^{-1} A_{i}(p)$ is a linear subspace of $\mathbb{R}^{N}$ for $i \in\{1, \ldots, n-1\}$ and $p \in P$. Then, the following statements are fulfilled:

(i) Forward convergence. For all $(p, x) \in P \times \mathbb{P}^{N-1}$, there exists an $i \in\{1, \ldots, n\}$ with

$$
\lim _{t \rightarrow \infty} d_{\mathbb{P}}\left(\mathbb{P} \Phi(t, p) x, M_{i}\left(\theta_{t} p\right)\right)=0
$$

(ii) Pushforward convergence. For all $p \in P$ and all functions $\gamma: \mathbb{T}^{+} \rightarrow \mathbb{P}^{N-1}$ with

$$
\liminf _{t \rightarrow \infty} d_{\mathbb{P}}\left(\gamma(t), \bigcup_{j=1}^{n} \partial A_{j}\left(\theta_{t} p\right)\right)>0,
$$

we have

$$
\lim _{t \rightarrow \infty} d_{\mathbb{P}}\left(\mathbb{P} \Phi\left(-t, \theta_{t} p\right) \gamma(t), \bigcup_{j=1}^{n} M_{j}(p)\right)=0
$$

PROOF. (i) This assertion is also valid for general Morse decompositions and was proved in Theorem 3.2.6.

(ii) Choose $(p, x) \in P \times \mathbb{P}^{N-1}$ arbitrarily. Then, there exists an $i \in\{1, \ldots, n\}$ such that

$$
x \in R_{i}^{*}(p) \quad \text { and } \quad x \notin R_{i-1}^{*}(p) .
$$


In case $x \in R_{i-1}(p)$, the above limit relation follows, since then $x \in M_{i}(p)$ and $M_{i}$ is invariant. We therefore assume $x \notin R_{i-1}(p)$ from now on. To obtain a contradiction, we also assume that there exist an $\varepsilon>0$ and a sequence $\left\{t_{n}\right\}_{n \in \mathbb{N}}$ in $\mathbb{R}$ with $\lim _{n \rightarrow \infty} t_{n}=\infty$ such that

$$
d_{\mathbb{P}}\left(\mathbb{P} \Phi\left(-t_{n}, p\right) x, M_{i}\left(\theta_{-t_{n}} p\right)\right) \geq \varepsilon \quad \text { for all } n \in \mathbb{N} .
$$

We define $C:=\mathbb{P}^{-1} M_{i}(p) \oplus \mathbb{P}^{-1}\{x\}$. Since $\lim _{t \rightarrow \infty} d_{\mathbb{P}}\left(\mathbb{P} \Phi(-t, p) x, R_{i-1}\left(\theta_{-t} p\right)\right)=0$ (cf. Theorem 3.1.5), Lemma 3.5.3 implies

$$
\lim _{n \rightarrow \infty} d_{\mathbb{P}}\left(\mathbb{P} \Phi\left(-t_{n}, p\right) \mathbb{P} C \mid R_{i-1}\left(\theta_{-t_{n}} p\right)\right)=0 .
$$

We define $\bar{C}:=\mathbb{P}^{-1} R_{i-1}(p) \oplus \mathbb{P}^{-1}\{x\}$. Then,

$$
\begin{aligned}
\operatorname{dim}\left(\bar{C} \cap \mathbb{P}^{-1} R_{i-1}^{*}(p)\right) & =\operatorname{dim} \bar{C}+\operatorname{dim} \mathbb{P}^{-1} R_{i-1}^{*}(p)-\operatorname{dim}\left(\bar{C}+\mathbb{P}^{-1} R_{i-1}^{*}(p)\right) \\
& =N+1-N=1 .
\end{aligned}
$$

Let $y=v+w$ be a nonzero element of $\bar{C} \cap \mathbb{P}^{-1} R_{i-1}^{*}(p)$ with $v \in \mathbb{P}^{-1}\{x\}$ and $w \in \mathbb{P}^{-1} R_{i-1}(p)$. Since $y$ and $v$ are in $\mathbb{P}^{-1} R_{i}^{*}(p), w$ is also an element of $\mathbb{P}^{-1} R_{i}^{*}(p)$. Hence, $w \in \mathbb{P}^{-1} M_{i}(p)$. This implies $y \in C$, and hence, we get from (3.9) the relation

$$
\lim _{n \rightarrow \infty} d_{\mathbb{P}}\left(\mathbb{P} \Phi\left(-t_{n}, p\right) \mathbb{P} y, R_{i-1}\left(\theta_{-t_{n}} p\right)\right)=0 .
$$

This is a contradiction, since $\mathbb{P} y \in R_{i-1}^{*}(p)$ and $R_{i-1}$ and $R_{i-1}^{*}$ are past isolated (cf. Theorem 3.1.5 (i)).

In our special situation, Proposition 3.1.6 can be generalized.

3.5.5 Proposition (Form of Nonuniqueness of the Morse Sets). Let $\left\{M_{1}, \ldots, M_{n}\right\}$ and $\left\{\hat{M}_{1}, \ldots, \hat{M}_{n}\right\}$ be past Morse decompositions obtained by the finite sequences of past repellers $R_{0} \supset \cdots \supset R_{n}$ and $\hat{R}_{0} \supset \cdots \supset \hat{R}_{n}$ such that $\mathbb{P}^{-1} R_{i}(p)$ and $\mathbb{P}^{-1} \hat{R}_{i}(p)$ are linear subspaces of $\mathbb{R}^{N}$ for $i \in\{1, \ldots, n-1\}$ and $p \in P$. We assume that

$$
R_{i}^{*}=\hat{R}_{i}^{*} \quad \text { for all } i \in\{1, \ldots, n-1\} .
$$

Then, the relation

$$
\lim _{t \rightarrow \infty} d_{\mathbb{P} H}\left(M_{i}\left(\theta_{-t} p\right), \hat{M}_{i}\left(\theta_{-t} p\right)\right)=0 \quad \text { for all } i \in\{1, \ldots, n\} \text { and } p \in P
$$

is fulfilled.

Let $\left\{M_{1}, \ldots, M_{n}\right\}$ and $\left\{\hat{M}_{1}, \ldots, \hat{M}_{n}\right\}$ be future Morse decompositions obtained by the finite sequences of future attractors $A_{0} \subset \cdots \subset A_{n}$ and $\hat{A}_{0} \subset \cdots \subset \hat{A}_{n}$ such that $\mathbb{P}^{-1} A_{i}(p)$ and $\mathbb{P}^{-1} \hat{A}_{i}(p)$ are linear subspaces of $\mathbb{R}^{N}$ for $i \in\{1, \ldots, n-1\}$ and $p \in P$. We assume that

$$
A_{i}^{*}=\hat{A}_{i}^{*} \quad \text { for all } i \in\{1, \ldots, n-1\} .
$$

Then, the relation

$$
\lim _{t \rightarrow \infty} d_{\mathbb{P} H}\left(M_{i}\left(\theta_{t} p\right), \hat{M}_{i}\left(\theta_{t} p\right)\right)=0 \quad \text { for all } i \in\{1, \ldots, n\} \text { and } p \in P
$$

is fulfilled. 
Proof. For $i \in\{1, n\}$, the above limit relation follows from $M_{1}=R_{1}^{*}=\hat{R}_{1}^{*}=\hat{M}_{1}$ and from Proposition 3.1.6, since $M_{n}=R_{n-1}$ and $\hat{M}_{n}=\hat{R}_{n-1}$. We argue negatively and assume w.l.o.g. that there exist an $i \in\{2, \ldots, n-1\}$ and a $p \in P$ such that

$$
\limsup _{t \rightarrow \infty} d_{\mathbb{P}}\left(\hat{M}_{i}\left(\theta_{-t} p\right) \mid M_{i}\left(\theta_{-t} p\right)\right)>0 .
$$

Since Proposition 3.1.6 implies that

$$
\lim _{t \rightarrow \infty} d_{\mathbb{P} H}\left(R_{i-1}\left(\theta_{-t} p\right), \hat{R}_{i-1}\left(\theta_{-t} p\right)\right)=0,
$$

and $\hat{R}_{i-1} \supset \hat{M}_{i}$, this means that there exist a $\gamma>0$ and sequences $\left\{t_{n}\right\}_{n \in \mathbb{N}}\left(\right.$ with $\left.\lim _{n \rightarrow \infty} t_{n}=\infty\right)$ and $\left\{x_{n}\right\}_{n \in \mathbb{N}}$ (with $x_{n} \in R_{i-1}\left(\theta_{-t_{n}} p\right) \backslash M_{i}\left(\theta_{-t_{n}} p\right)$ ) such that

$$
d_{\mathbb{P}}\left(x_{n}, M_{i}\left(\theta_{-t_{n}} p\right)\right) \geq \gamma \quad \text { for all } n \in \mathbb{N}
$$

and

$$
\lim _{n \rightarrow \infty} d_{\mathbb{P}}\left(x_{n}, \hat{M}_{i}\left(\theta_{-t_{n}} p\right)\right)=0 \text {. }
$$

The last formula implies $\lim _{n \rightarrow \infty} d_{\mathbb{P}}\left(x_{n}, R_{i}^{*}\left(\theta_{-t_{n}} p\right)\right)=0$. We define

$$
C_{n}:=\mathbb{P}^{-1} M_{i}\left(\theta_{-t_{n}} p\right) \oplus \mathbb{P}^{-1}\left\{x_{n}\right\} \quad \text { for all } n \in \mathbb{N} \text {. }
$$

Due to Lemma 3.5.3, the relation

$$
\lim _{n \rightarrow \infty} d_{\mathbb{P}}\left(\mathbb{P} C_{n} \mid R_{i}^{*}\left(\theta_{-t_{n}} p\right)\right)=0
$$

holds. Since $R_{i}^{*}$ is a past attractor, we thus get

$$
\lim _{n \rightarrow \infty} d_{\mathbb{P}}\left(\mathbb{P} \Phi\left(t_{n}, \theta_{-t_{n}} p\right) \mathbb{P} C_{n} \mid R_{i}^{*}(p)\right)=0 .
$$

Due to Lemma A.3.2, we have $d_{\mathbb{P}}\left(\mathbb{P} \Phi\left(t_{n}, \theta_{-t_{n}} p\right) \mathbb{P} C_{n} \mid M_{i}(p)\right)=\sqrt{2}$ for all $n \in \mathbb{N}$, since $\mathbb{P}^{-1} \mathbb{P} \Phi\left(t_{n}, \theta_{-t_{n}} p\right) \mathbb{P} C_{n}$ has a higher dimension than $\mathbb{P}^{-1} M_{i}(p)$. This means that there exists a sequence $\left\{y_{n}\right\}_{n \in \mathbb{N}}$ with

$$
y_{n} \in \mathbb{P} \Phi\left(t_{n}, \theta_{-t_{n}} p\right) \mathbb{P} C_{n} \quad \text { and } \quad d_{\mathbb{P}}\left(y_{n}, M_{i}(p)\right) \geq 1 \quad \text { for all } n \in \mathbb{N} .
$$

Since $y_{n} \in R_{i-1}(p)$ for all $n \in \mathbb{N}$, we assume w.l.o.g. that this sequence is convergent with limit $y \in R_{i-1}(p)$. Due to (3.10), we also have $y \in R_{i}^{*}(p)$. Hence, $y \in M_{i}(p)$, and this contradicts (3.11).

For the rest of this chapter, attention is restricted to the situation $P=\mathbb{T}$ and $\theta(t, s)=t+s$ for all $t, s \in \mathbb{T}$. As described in Section 1.2, this setting includes arbitrary nonautonomous differential and difference equations. Under this assumption, an analogon to the THEOREM OF SELGRADE (see Selgrade [163, Theorem 9.7] and Colonius \& Kliemann [49, Theorem 5.2.5]) can be proved.

3.5.6 Theorem (Finest Nonautonomous Morse Decomposition). We suppose that $P=\mathbb{T}$ and $\theta(t, s)=t+s$ for all $t, s \in \mathbb{T}$. Then, the following statements are fulfilled:

(i) There exists a finest past Morse decomposition $\left\{M_{1}, \ldots, M_{n}\right\}$, i.e., any other past Morse decomposition $\left\{\tilde{M}_{1}, \ldots, \tilde{M}_{m}\right\}$ fulfills 


$$
\lim _{t \rightarrow \infty} d_{\mathbb{P}}\left(\bigcup_{i=1}^{n} M_{i}(-t) \mid \bigcup_{i=1}^{m} \tilde{M}_{i}(-t)\right)=0 .
$$

Moreover, we have $n \leq N$, and the following decomposition in a Whitney sum holds (cf. the definition on p. 74):

$$
\mathbb{P}^{-1} M_{1} \oplus \cdots \oplus \mathbb{P}^{-1} M_{n}=\mathbb{T} \times \mathbb{R}^{N}
$$

(ii) There exists a finest future Morse decomposition $\left\{M_{1}, \ldots, M_{n}\right\}$, i.e., any other future Morse decomposition $\left\{\tilde{M}_{1}, \ldots, \tilde{M}_{m}\right\}$ fulfills

$$
\lim _{t \rightarrow \infty} d_{\mathbb{P}}\left(\bigcup_{i=1}^{n} M_{i}(t) \mid \bigcup_{i=1}^{m} \tilde{M}_{i}(t)\right)=0 .
$$

Moreover, we have $n \leq N$, and the following decomposition in a Whitney sum holds:

$$
\mathbb{P}^{-1} M_{1} \oplus \cdots \oplus \mathbb{P}^{-1} M_{n}=\mathbb{T} \times \mathbb{R}^{N}
$$

PROOF. First, we prove that any past attractors $A$ and $\hat{A}$ either fulfill

$$
A \subset \hat{A} \text { or } A \supset \hat{A}
$$

Supposing the contrary, due to $P=\mathbb{T}$, there exist a $\tau \in \mathbb{T}$ and elements

$$
x \in \mathbb{S}^{N-1} \cap\left(\mathbb{P}^{-1} A(\tau) \backslash \mathbb{P}^{-1} \hat{A}(\tau)\right) \text { and } \hat{x} \in \mathbb{S}^{N-1} \cap\left(\mathbb{P}^{-1} \hat{A}(\tau) \backslash \mathbb{P}^{-1} A(\tau)\right) .
$$

Because of Proposition 3.5.1, we obtain

$$
\lim _{t \rightarrow \infty} \frac{\|\Phi(-t, \tau) x\|}{\|\Phi(-t, \tau) \hat{x}\|}=0 \quad \text { and } \quad \lim _{t \rightarrow \infty} \frac{\|\Phi(-t, \tau) \hat{x}\|}{\|\Phi(-t, \tau) x\|}=0 .
$$

This is a contradiction. Proposition 3.5.1 also implies that the fibers of past attractors correspond to linear subspaces. Thus, there are at most $N+1$ past attractors of $(\theta, \mathbb{P} \Phi)$, namely

$$
\emptyset=A_{0} \subsetneq A_{1} \subsetneq \cdots \subsetneq A_{n}=\mathbb{T} \times \mathbb{P}^{N-1}
$$

with $n \leq N$. Due to Proposition 3.5.2, it is possible to choose a sequence of past repellers $\mathbb{T} \times \mathbb{P}^{N-1}=R_{0} \supsetneq R_{1} \supsetneq \cdots \supsetneq R_{n}=\emptyset$ such that $R_{i}^{*}=A_{i}$ for $i \in\{0, \ldots, n\}$. We denote by $\left\{M_{1}, \ldots, M_{n}\right\}$ the corresponding past Morse decomposition. Let $\left\{\tilde{M}_{1}, \ldots, \tilde{M}_{m}\right\}$ be another past Morse decomposition, obtained by the sequence $\mathbb{T} \times \mathbb{P}^{N-1}=\tilde{R}_{0} \supsetneq \tilde{R}_{1} \supsetneq \cdots \supsetneq \tilde{R}_{m}=\emptyset$ of past repellers. Then, for each $i \in\{0, \ldots, m\}$, there exists an $n_{i} \in\{0, \ldots, n\}$ such that $\tilde{R}_{i}^{*}=A_{n_{i}}$. We consider now the past Morse decomposition $\left\{\hat{M}_{1}, \ldots, \hat{M}_{n}\right\}$ which is obtained by the past repellers $R_{n_{0}}, \ldots, R_{n_{m}}$. Due to Proposition 3.5.5, we have

$$
\lim _{t \rightarrow \infty} d_{\mathbb{P} H}\left(\bigcup_{i=1}^{m} \hat{M}_{i}(-t), \bigcup_{i=1}^{m} \tilde{M}_{i}(-t)\right)=0 .
$$

Moreover, it is easy to see that $\cup_{i=1}^{n} M_{i} \subset \cup_{i=1}^{m} \hat{M}_{i}$ holds, and this finishes the proof of the first assertion of this theorem. To show

$$
\mathbb{P}^{-1} M_{1} \oplus \cdots \oplus \mathbb{P}^{-1} M_{n}=\mathbb{T} \times \mathbb{R}^{N},
$$


we first note that for $1 \leq i<j \leq n$, we have $\mathbb{P}^{-1} M_{i} \cap \mathbb{P}^{-1} M_{j}=\mathbb{T} \times\{0\}$ (cf. Proposition 3.2.3). Furthermore, Proposition 3.5.2 and Lemma A.2.3 implies

$$
\begin{aligned}
\mathbb{T} \times \mathbb{R}^{N} & =\mathbb{P}^{-1} R_{1}^{*}+\mathbb{P}^{-1} R_{1} \\
& =\mathbb{P}^{-1} M_{1}+\left(\mathbb{P}^{-1} R_{1} \cap\left(\mathbb{P}^{-1} R_{2}^{*}+\mathbb{P}^{-1} R_{2}\right)\right) \\
& =\mathbb{P}^{-1} M_{1}+\left(\mathbb{P}^{-1} R_{1} \cap \mathbb{P}^{-1} R_{2}^{*}\right)+\mathbb{P}^{-1} R_{2} \\
& =\mathbb{P}^{-1} M_{1}+\mathbb{P}^{-1} M_{2}+\mathbb{P}^{-1} R_{2} .
\end{aligned}
$$

It follows inductively that

$$
\mathbb{T} \times \mathbb{R}^{N}=\mathbb{P}^{-1} M_{1}+\cdots+\mathbb{P}^{-1} M_{n}+\mathbb{P}^{-1} R_{n}=\mathbb{P}^{-1} M_{1}+\cdots+\mathbb{P}^{-1} M_{n} .
$$

This finishes the proof of this theorem.

3.5.7 Remark. A finest past Morse decomposition $\left\{M_{1}, \ldots, M_{n}\right\}$ is not uniquely determined, but it follows directly from the above theorem that any other finest Morse decomposition $\left\{\tilde{M}_{1}, \ldots, \tilde{M}_{m}\right\}$ satisfies

$$
\lim _{t \rightarrow \infty} d_{\mathbb{P} H}\left(\bigcup_{i=1}^{n} M_{i}(-t), \bigcup_{i=1}^{m} \tilde{M}_{i}(-t)\right)=0 .
$$

Moreover, the relation $n=m$ is fulfilled. A similar statement holds for finest future Morse decompositions. 



\section{CHAPTER 4}

\section{LINEAR SYSTEMS}

In the qualitative theory, the study of linear systems is very important, since a comprehensive analysis of nonlinear systems via perturbation techniques requires linear theory. This is due to the fact that in many cases, stability properties of solutions can be derived from the linearization along the solution, the so-called variational equation. In this chapter, methods are provided for the analysis of linear systems with respect to the notions of attractivity and repulsivity which have been introduced in Chapter 2.

Throughout this chapter, let $\left(\theta: \mathbb{T} \times P \rightarrow P, \varphi: \mathbb{T} \times P \times \mathbb{R}^{N} \rightarrow \mathbb{R}^{N}\right)$ be a linear nonautonomous dynamical system, i.e.,

$$
\varphi(t, p)(\alpha x+\beta y)=\alpha \varphi(t, p) x+\beta \varphi(t, p) y \quad \text { for all } \alpha, \beta \in \mathbb{R}, t \in \mathbb{T}, p \in P \text { and } x, y \in \mathbb{R}^{N} .
$$

We suppose that $(\theta, \varphi)$ is invertible, i.e., $\mathbb{T}=\mathbb{R}$ or $\mathbb{T}=\mathbb{Z}$. Moreover, let $\Phi: \mathbb{T} \times P \rightarrow \mathbb{R}^{N \times N}$ be the matrix function with $\Phi(t, p) x=\varphi(t, p, x)$ for all $t \in \mathbb{T}, p \in P$ and $x \in \mathbb{R}^{N}$.

\subsection{Notions of Dichotomy}

In this section, several notions of dichotomy are introduced for the different time domains. The classical concept of exponential dichotomy for nonautonomous linear differential equations has been established by PERRON $[129,130]$ in the late 1920s. In the sequel, many authors developed the theory; for fundamental work on this topic, we refer to COPPEL [54], DALECKIĬ \& KREIN [60], Massera \& SchäFfer [117], PAlmer [124, 125, 126] and SACKer \& SEll [150, 151, 152, 149] (see also PAPASCHINOPOULOS [128] for difference equations). The noninvertible case is treated in Henry [78, Section 7.6], Kalkbrenner [88], Aulbach \& Kalkbrenner [15] and Aulbach \& Siegmund [20].

4.1.1 Definition (Linear Nonautonomous Invariant Manifold). An invariant nonautonomous set $M \subset P \times \mathbb{R}^{N}$ is called linear nonautonomous invariant manifold of $(\theta, \varphi)$ if $M(p)$ is a linear subspace of $\mathbb{R}^{N}$ for all $p \in P$.

Given linear nonautonomous invariant manifolds $M_{1}, M_{2}$ of $(\theta, \varphi)$, the sets

$$
\begin{aligned}
& M_{1} \cap M_{2}:=\left\{(p, \xi) \in P \times \mathbb{R}^{N}: \xi \in M_{1}(p) \cap M_{2}(p)\right\} \quad \text { and } \\
& M_{1}+M_{2}:=\left\{(p, \xi) \in P \times \mathbb{R}^{N}: \xi \in M_{1}(p)+M_{2}(p)\right\}
\end{aligned}
$$


are also linear nonautonomous invariant manifolds of $(\theta, \varphi)$. A finite sum $M_{1}+\cdots+M_{n}$ of linear nonautonomous invariant manifolds is called Whitney sum $M_{1} \oplus \cdots \oplus M_{n}$ if the relation $M_{i} \cap M_{j}=P \times\{0\}$ is satisfied for $i \neq j$.

Linear nonautonomous invariant manifolds can be described via invariant projectors.

4.1.2 Definition (InVARiant Projector). An invariant projector of $(\theta, \varphi)$ is a function $Q: P \rightarrow \mathbb{R}^{N \times N}$ with

$$
\begin{aligned}
Q(p) & =Q(p)^{2} & & \text { for all } p \\
Q\left(\theta_{t} p\right) \Phi(t, p) & =\Phi(t, p) Q(p) & & \text { for all } p \in P \text { and } t \in \mathbb{T} .
\end{aligned}
$$

4.1.3 REMARK. In case the NDS $(\theta, \varphi)$ is a topological skew product flow, i.e., $P$ is a topological space, one usually supposes additionally that an invariant projector is continuous (see, e.g., SACKER \& SELL [150]).

The range

$$
\mathcal{R}(Q):=\left\{(p, \xi) \in P \times \mathbb{R}^{N}: \xi \in \mathcal{R}(Q(p))\right\}
$$

and the null space

$$
\mathcal{N}(Q):=\left\{(p, \xi) \in P \times \mathbb{R}^{N}: \xi \in \mathcal{N}(Q(p))\right\}
$$

of an invariant projector $Q$ are linear nonautonomous invariant manifolds of $(\theta, \varphi)$ such that $\mathcal{R}(Q) \oplus \mathcal{N}(Q)=P \times \mathbb{R}^{N}$.

Next, several notions of dichotomy are introduced for the linear system $(\theta, \varphi)$.

4.1.4 Definition (Notions of Dichotomy). Let $Q: P \rightarrow \mathbb{R}^{N \times N}$ be an invariant projector of $(\theta, \varphi)$.

(i) We say, $(\theta, \varphi)$ admits a past exponential dichotomy with constants $\alpha>0, K \geq 1$ and projector $Q$ if for all $p \in P$, there exists a $\hat{p} \in[p]$ with

$$
\begin{aligned}
&\left\|\Phi\left(t, \theta_{-\tau} \hat{p}\right) Q\left(\theta_{-\tau} \hat{p}\right)\right\| \leq K e^{-\alpha t} \quad \text { for all } \tau \geq 0 \text { and } 0 \leq t \leq \tau, \\
&\left\|\Phi\left(-t, \theta_{-\tau} \hat{p}\right)\left(\mathbb{1}-Q\left(\theta_{-\tau} \hat{p}\right)\right)\right\| \leq K e^{-\alpha t} \quad \text { for all } \tau \geq 0 \text { and } 0 \leq t
\end{aligned}
$$

(ii) We say, $(\theta, \varphi)$ admits a future exponential dichotomy with constants $\alpha>0, K \geq 1$ and projector $Q$ if for all $p \in P$, there exists a $\hat{p} \in[p]$ with

$$
\begin{aligned}
\left\|\Phi\left(t, \theta_{\tau} \hat{p}\right) Q\left(\theta_{\tau} \hat{p}\right)\right\| \leq K e^{-\alpha t} & \text { for all } \tau \geq 0 \text { and } 0 \leq t, \\
\left\|\Phi\left(-t, \theta_{\tau} \hat{p}\right)\left(\mathbb{1}-Q\left(\theta_{\tau} \hat{p}\right)\right)\right\| \leq K e^{-\alpha t} & \text { for all } \tau \geq 0 \text { and } 0 \leq t \leq \tau .
\end{aligned}
$$

(iii) We say, $(\theta, \varphi)$ admits an all-time exponential dichotomy with constants $\alpha>0, K \geq 1$ and projector $Q$ if for all $p \in P$, we have

$$
\begin{aligned}
\|\Phi(t, p) Q(p)\| \leq K e^{-\alpha t} & \text { for all } t \geq 0 \\
\|\Phi(-t, p)(\mathbb{1}-Q(p))\| \leq K e^{-\alpha t} & \text { for all } t \geq 0
\end{aligned}
$$

(iv) Given $p \in P$ and $T \in \mathbb{T}^{+}$, we say, $(\theta, \varphi)$ admits a $(p, T)$-dichotomy with projector $Q$ if we have 


$$
\begin{array}{rlrl}
\|\Phi(T, p) \xi\| & <\|\xi\| & & \text { for all } 0 \neq \xi \in \mathcal{R}(Q(p)), \\
\left\|\Phi\left(-T, \theta_{T} p\right) \xi\right\| & <\|\xi\| & \text { for all } 0 \neq \xi \in \mathcal{N}\left(Q\left(\theta_{T} p\right)\right) .
\end{array}
$$

Having these definitions at hand, some remarks are in order.

\subsubsection{REMARKS.}

(i) In the literature (see the references cited in the introduction of this section), an all-time exponential dichotomy is simply called exponential dichotomy.

(ii) In case the $\operatorname{NDS}(\theta, \varphi)$ is generated by a nonautonomous differential or difference equation, i.e., $P=\mathbb{T}$, a past or future exponential dichotomy is called exponential dichotomy on half line $\mathbb{R}_{0}^{-}, \mathbb{Z}_{0}^{-}$or $\mathbb{R}_{0}^{+}, \mathbb{Z}_{0}^{+}$, respectively (see, e.g., COPPEL [54] and Proposition 4.2.1).

(iii) In contrast to past, future or all-time exponential dichotomies, the notion of $(p, T)$ dichotomy is not invariant with respect to a change of the norm to an equivalent norm (cf. also Remark 2.1.10).

(iv) In the scalar case $(N=1),(\theta, \varphi)$ admits a $(p, T)$-dichotomy if and only if $|\Phi(T, p)| \neq 1$.

In the following proposition, the relationship between the above introduced notions of dichotomies is examined.

4.1.6 PROPOSITION. The following statements are fulfilled:

(i) If $(\theta, \varphi)$ admits an all-time exponential dichotomy, then it also admits a past exponential dichotomy and a future exponential dichotomy.

(ii) Suppose, $(\theta, \varphi)$ is generated by a nonautonomous differential or difference equation, i.e., $P=\mathbb{T}$, and $(\theta, \varphi)$ admits a past exponential dichotomy and a future exponential dichotomy with the same invariant projector $Q: \mathbb{T} \rightarrow \mathbb{R}^{N \times N}$. Then, $(\theta, \varphi)$ also admits an all-time exponential dichotomy.

Proof. Statement (i) is obvious; for (ii), see Coppel [54, p. 19].

4.1.7 Definition (Nonhyperbolic Dichotomies). Let $\gamma \in \mathbb{R}$, and consider the linear $\operatorname{NDS}\left(\theta, \varphi_{\gamma}\right)$, defined by

$$
\varphi_{\gamma}(t, p, x):=e^{-\gamma t} \varphi(t, p, x) \text { for all } t \in \mathbb{T}, p \in P \text { and } x \in \mathbb{R}^{N} .
$$

We say, $(\theta, \varphi)$ admits a nonhyperbolic past exponential (future exponential, all-time exponential, $(p, T)$-, respectively) dichotomy with growth rate $\gamma$, constants $\alpha>0, K \geq 1$ and projector $Q$ if $\left(\theta, \varphi_{\gamma}\right)$ admits a past exponential (future exponential, all-time exponential, ( $p, T)$-, respectively) dichotomy with constants $\alpha>0, K \geq 1$ and projector $Q$.

4.1.8 REMARK. The NDS $(\theta, \varphi)$ admits a nonhyperbolic past exponential (future exponential, all-time exponential, $(p, T)$-, respectively) dichotomy with growth rate $\gamma=0$ if and only if it admits a past exponential (future exponential, all-time exponential, $(p, T)$, respectively) dichotomy. 
4.1.9 Lemma (CRiteria for Nonhyperbolic Dichotomies). Suppose, $(\theta, \varphi)$ admits a nonhyperbolic past exponential (future exponential, all-time exponential, $(p, T)$-, respectively) dichotomy with growth rate $\gamma$ and projector $Q_{\gamma}$. Then, the following statements are fulfilled:

(i) If $Q_{\gamma} \equiv \mathbb{1}$, then $(\theta, \varphi)$ admits a nonhyperbolic past exponential (future exponential, all-time exponential, $(p, T)$-, respectively) dichotomy with growth rate $\zeta$ and projector $Q_{\zeta} \equiv \mathbb{1}$ for all $\zeta>\gamma$.

(ii) If $Q_{\gamma} \equiv \mathbb{O}$, then $(\theta, \varphi)$ admits a nonhyperbolic past exponential (future exponential, all-time exponential, $(p, T)$-, respectively) dichotomy with growth rate $\zeta$ and projector $Q_{\zeta} \equiv \mathbb{O}$ for all $\zeta<\gamma$.

PROOF. The assertions follow directly from the monotonicity of the exponential function.

We make use of the following equivalent characterizations of nonhyperbolic dichotomies.

4.1.10 Proposition (EQuivalent Characterizations of Nonhyperbolic DiCHOTOMiEs). Let $Q: P \rightarrow \mathbb{R}^{N \times N}$ be an invariant projector of $(\theta, \varphi)$. Then, the following statements are fulfilled:

(i) $(\theta, \varphi)$ admits a nonhyperbolic past exponential dichotomy with growth rate $\gamma \in \mathbb{R}$, constants $\alpha>0, K \geq 1$ and projector $Q$ if and only if for all $p \in P$, there exists a $\hat{p} \in[p]$ with

$$
\begin{aligned}
\left\|\Phi\left(t, \theta_{-\tau} \hat{p}\right) Q\left(\theta_{-\tau} \hat{p}\right)\right\| & \leq K e^{(\gamma-\alpha) t} & & \text { for all } \tau \geq 0 \text { and } 0 \leq t \leq \tau, \\
\left\|\Phi\left(-t, \theta_{-\tau} \hat{p}\right)\left(\mathbb{1}-Q\left(\theta_{-\tau} \hat{p}\right)\right)\right\| & \leq K e^{-(\gamma+\alpha) t} & & \text { for all } \tau \geq 0 \text { and } 0 \leq t .
\end{aligned}
$$

(ii) $(\theta, \varphi)$ admits a nonhyperbolic future exponential dichotomy with growth rate $\gamma \in \mathbb{R}$, constants $\alpha>0, K \geq 1$ and projector $Q$ if and only if for all $p \in P$, there exists a $\hat{p} \in[p]$ with

$$
\begin{array}{cl}
\left\|\Phi\left(t, \theta_{\tau} \hat{p}\right) Q\left(\theta_{\tau} \hat{p}\right)\right\| \leq K e^{(\gamma-\alpha) t} & \text { for all } \tau \geq 0 \text { and } 0 \leq t, \\
\left\|\Phi\left(-t, \theta_{\tau} \hat{p}\right)\left(\mathbb{1}-Q\left(\theta_{\tau} \hat{p}\right)\right)\right\| \leq K e^{-(\gamma+\alpha) t} & \text { for all } \tau \geq 0 \text { and } 0 \leq t \leq \tau .
\end{array}
$$

(iii) $(\theta, \varphi)$ admits a nonhyperbolic all-time exponential dichotomy with growth rate $\gamma \in \mathbb{R}$, constants $\alpha>0, K \geq 1$ and projector $Q$ if and only if for all $p \in P$, we have

$$
\begin{aligned}
\|\Phi(t, p) Q(p)\| & \leq K e^{(\gamma-\alpha) t} & & \text { for all } t \geq 0, \\
\|\Phi(-t, p)(\mathbb{1}-Q(p))\| & \leq K e^{-(\gamma+\alpha) t} & & \text { for all } t \geq 0 .
\end{aligned}
$$

(iv) Given $p \in P$ and $T \in \mathbb{T}^{+},(\theta, \varphi)$ admits a nonhyperbolic $(p, T)$-dichotomy with growth rate $\gamma \in \mathbb{R}$ and projector $Q$ if and only if we have

$$
\begin{array}{cl}
\|\varphi(T, p) \xi\|<e^{\gamma T}\|\xi\| & \text { for all } 0 \neq \xi \in \mathcal{R}(Q(p)), \\
\left\|\varphi\left(-T, \theta_{T} p\right) \xi\right\|<e^{-\gamma T}\|\xi\| & \text { for all } 0 \neq \xi \in \mathcal{N}\left(Q\left(\theta_{T} p\right)\right) .
\end{array}
$$


For $\gamma \in \mathbb{R}$, we define

$$
\mathcal{S}_{\gamma}:=\left\{(p, \xi) \in P \times \mathbb{R}^{N}: \Phi(\cdot, p) \xi \text { is } \gamma^{+} \text {-quasibounded }\right\}
$$

and

$$
\mathcal{U}_{\gamma}:=\left\{(p, \xi) \in P \times \mathbb{R}^{N}: \Phi(\cdot, p) \xi \text { is } \gamma^{-} \text {-quasibounded }\right\} .
$$

It is obvious that $\mathcal{S}_{\gamma}$ and $\mathcal{U}_{\gamma}$ are linear nonautonomous invariant manifolds of $(\theta, \varphi)$. Given $\gamma \leq \zeta$, the relations $\mathcal{S}_{\gamma} \subset \mathcal{S}_{\zeta}$ and $\mathcal{U}_{\gamma} \supset \mathcal{U}_{\zeta}$ are fulfilled.

We now discuss the important relationship between the projectors of nonhyperbolic exponential dichotomies with growth rate $\gamma$ and the sets $\mathcal{S}_{\gamma}$ and $\mathcal{U}_{\gamma}$.

4.1.11 Proposition (DynAmical Properties). If $(\theta, \varphi)$ admits a nonhyperbolic past exponential dichotomy with growth rate $\gamma$, constants $\alpha>0, K \geq 1$ and projector $Q$, then we have $\mathcal{N}(Q)=\mathcal{U}_{\gamma}$, and for all $p \in P$, there exists a $\hat{p} \in[p]$ with

$$
\left\|\Phi\left(t, \theta_{-\tau} \hat{p}\right) \xi\right\| \leq K\|\xi\| e^{\gamma t} \quad \text { for all } \tau \geq 0,0 \leq t \leq \tau \text { and } \xi \in \mathcal{R}\left(Q\left(\theta_{-\tau} \hat{p}\right)\right) \text {. }
$$

If $(\theta, \varphi)$ admits a nonhyperbolic future exponential dichotomy with growth rate $\gamma$, constants $\alpha>0, K \geq 1$ and projector $Q$, then we have $\mathcal{R}(Q)=\mathcal{S}_{\gamma}$, and for all $p \in P$, there exists a $\hat{p} \in[p]$ with

$$
\left\|\Phi\left(-t, \theta_{\tau} \hat{p}\right) \xi\right\| \leq K\|\xi\| e^{-\gamma t} \quad \text { for all } \tau \geq 0,0 \leq t \leq \tau \text { and } \xi \in \mathcal{N}\left(Q\left(\theta_{\tau} \hat{p}\right)\right) .
$$

If $(\theta, \varphi)$ admits a nonhyperbolic all-time exponential dichotomy with growth rate $\gamma$ and projector $Q$, then $\mathcal{N}(Q)=\mathcal{U}_{\gamma}$ and $\mathcal{R}(Q)=\mathcal{S}_{\gamma}$ are fulfilled.

Proof. Suppose, $(\theta, \varphi)$ admits a nonhyperbolic past exponential dichotomy with growth rate $\gamma$, constants $\alpha>0, K \geq 1$ and projector $Q$. Due to Proposition 4.1.10, for given $p \in P$, there exists a $\hat{p} \in[p]$ with

$$
\begin{aligned}
\left\|\Phi\left(t, \theta_{-\tau} \hat{p}\right) Q\left(\theta_{-\tau} \hat{p}\right)\right\| & \leq K e^{(\gamma-\alpha) t} & & \text { for all } \tau \geq 0 \text { and } 0 \leq t \leq \tau, \\
\left\|\Phi\left(-t, \theta_{-\tau} \hat{p}\right)\left(\mathbb{1}-Q\left(\theta_{-\tau} \hat{p}\right)\right)\right\| & \leq K e^{-(\gamma+\alpha) t} & & \text { for all } \tau \geq 0 \text { and } 0 \leq t
\end{aligned}
$$

The first inequality implies (4.1). Choose $\hat{t} \in \mathbb{T}$ such that $\theta_{\hat{t}} p=\hat{p}$. We now prove the relation $\mathcal{N}(Q)=\mathcal{U}_{\gamma}$.

() We choose $(p, \xi) \in \mathcal{U}_{\gamma}$ arbitrarily. This implies $\left\|\Phi\left(-t, \theta_{\hat{t}} p\right) \Phi(\hat{t}, p) \xi\right\| \leq C e^{-\gamma t}$ for all $t \geq 0$ with some real constant $C>0$. We write $\Phi(\hat{t}, p) \xi=\xi_{1}+\xi_{2}$ with $\xi_{1} \in \mathcal{R}(Q(\hat{p}))$ and $\xi_{2} \in \mathcal{N}(Q(\hat{p}))$. Hence, for all $t \geq 0$, we get

$$
\begin{aligned}
\left\|\xi_{1}\right\| & =\left\|\Phi\left(t, \theta_{-t} \hat{p}\right) \Phi(-t, \hat{p}) Q(\hat{p}) \Phi(\hat{t}, p) \xi\right\|=\left\|\Phi\left(t, \theta_{-t} \hat{p}\right) Q\left(\theta_{-t} \hat{p}\right) \Phi(-t, \hat{p}) \Phi(\hat{t}, p) \xi\right\| \\
& \leq K e^{(\gamma-\alpha) t}\|\Phi(-t, \hat{p}) \Phi(\hat{t}, p) \xi\| \leq C K e^{(\gamma-\alpha) t} e^{-\gamma t}=C K e^{-\alpha t} .
\end{aligned}
$$

The right hand side of this inequality converges to zero in the limit $t \rightarrow \infty$. Therefore, $\xi_{1}=0$, and $\Phi(\hat{t}, p) \xi \in \mathcal{N}(Q(\hat{p}))$. Due to the invariance of $\mathcal{N}(Q)$, we finally obtain $(p, \xi) \in \mathcal{N}(Q)$.

$(\subseteq)$ We choose $(p, \xi) \in \mathcal{N}(Q)$. Thus, for all $t \geq 0$, the relation

$$
\|\Phi(-t, \hat{p}) \Phi(\hat{t}, p) \xi\|=\|\Phi(-t, \hat{p})(\mathbb{1}-Q(\hat{p})) \Phi(\hat{t}, p) \xi\| \leq K e^{-(\gamma+\alpha) t}\|\Phi(\hat{t}, p) \xi\|
$$

is fulfilled. This means that $\Phi(\cdot, p) \xi$ is $\gamma^{-}$-quasibounded, i.e., $(p, \xi) \in \mathcal{U}_{\gamma}$.

The assertions concerning the future exponential dichotomy are treated analogously. In case $(\theta, \varphi)$ 
admits an all-time exponential dichotomy, Proposition 4.1.6 (i) yields that $(\theta, \varphi)$ also admits a past exponential dichotomy and a future exponential dichotomy. Hence, we obtain $\mathcal{N}(Q)=\mathcal{U}_{\gamma}$ and $\mathcal{R}(Q)=\mathcal{S}_{\gamma}$.

4.1.12 REMARK. According to this proposition, an invariant projector is uniquely determined only in case of a nonhyperbolic all-time exponential dichotomy. In addition, the null space of a projector of a past exponential dichotomy and the range of a projector of a future exponential dichotomy are uniquely determined. For further information about the kind of nonuniqueness of ranges of projectors of past exponential dichotomies and null spaces of projectors of future exponential dichotomies, we refer to Lemma 4.2.4.

This section is concluded by pointing out several evidences that the notions of dichotomy are consistent to the concepts of attractivity and repulsivity.

4.1.13 Theorem (Nonhyperbolic Dichotomies AND the Notions of ATtraCTIVITY AND REPUlSIVITY). Suppose, $(\theta, \varphi)$ admits a nonhyperbolic past exponential (future exponential, all-time exponential, $(p, T)$-, respectively) dichotomy with growth rate $\gamma$ and invariant projector $Q$. Then, the following statements are fulfilled:

(i) If $\gamma \leq 0$ and $\mathrm{rk} Q(\hat{p}) \geq 1$ for all $\hat{p} \in P$, then every trivial solution of $(\theta, \varphi)$ is not past (future, all-time, $(p, T)-$, respectively) repulsive.

(ii) If $\gamma \geq 0$ and $\mathrm{rk} Q(\hat{p}) \leq N-1$ for all $\hat{p} \in P$, then every trivial solution of $(\theta, \varphi)$ is not past (future, all-time, $(p, T)$-, respectively) attractive.

(iii) If $\gamma \leq 0$ and $Q \equiv \mathbb{1}$, then every trivial solution of $(\theta, \varphi)$ is past (future, all-time, $(p, T)$-, respectively) attractive with $\mathfrak{A}_{0}=\infty$.

(iv) If $\gamma \geq 0$ and $Q \equiv \mathbb{0}$, then every trivial solution of $(\theta, \varphi)$ is past (future, all-time, ( $p, T)$-, respectively) repulsive with $\mathfrak{R}_{0}=\infty$.

PROOF. These assertions are direct consequences of Proposition 4.1.10 and 4.1.11.

For the rest of this section, the studies are concentrated on the induced nonautonomous dynamical system $(\theta, \mathbb{P} \Phi)$ on the real projective space $\mathbb{P}^{N-1}$ (cf. Section 3.5).

4.1.14 LEMMA. The following statements are fulfilled:

(i) We suppose that $(\theta, \varphi)$ admits a nonhyperbolic past exponential dichotomy with invariant projector $Q$. Then, there exists a $\beta>0$ such that for all $p \in P$, there exists a $\hat{p} \in[p]$ with

$$
U_{\beta}\left(\mathbb{P} \mathcal{R}\left(Q\left(\theta_{-t} \hat{p}\right)\right)\right) \cap U_{\beta}\left(\mathbb{P N}\left(Q\left(\theta_{-t} \hat{p}\right)\right)\right)=\emptyset \quad \text { for all } t \geq 0
$$

(i.e., $\mathbb{P} \mathcal{R}(Q)$ and $\mathbb{P} \mathcal{N}(Q)$ are past isolated). Moreover, for all $p \in P$ and compact sets $C \subset \mathbb{S}^{N-1} \backslash \mathcal{N}(Q(p))$, we have

$$
\lim _{t \rightarrow \infty} \frac{\sup _{v \in \mathbb{S}^{N-1} \cap \mathcal{N}(Q(p))}\|\Phi(-t, p) v\|}{\inf _{w \in C}\|\Phi(-t, p) w\|}=0 .
$$

(ii) We suppose that $(\theta, \varphi)$ admits a nonhyperbolic future exponential dichotomy with 
invariant projector $Q$. Then, there exists a $\beta>0$ such that for all $p \in P$, there exists a $\hat{p} \in[p]$ with

$$
U_{\beta}\left(\mathbb{P R}\left(Q\left(\theta_{t} \hat{p}\right)\right)\right) \cap U_{\beta}\left(\mathbb{P} \mathcal{N}\left(Q\left(\theta_{t} \hat{p}\right)\right)\right)=\emptyset \quad \text { for all } t \geq 0
$$

(i.e., $\mathbb{P} \mathcal{R}(Q)$ and $\mathbb{P} \mathcal{N}(Q)$ are future isolated). Moreover, for all $p \in P$ and compact sets $C \subset \mathbb{S}^{N-1} \backslash \mathcal{R}(Q(p))$, we have

$$
\lim _{t \rightarrow \infty} \frac{\sup _{v \in \mathbb{S}^{N-1} \cap \mathcal{R}(Q(p))}\|\Phi(t, p) v\|}{\inf _{w \in C}\|\Phi(t, p) w\|}=0 .
$$

(iii) We suppose that $(\theta, \varphi)$ admits a nonhyperbolic all-time exponential dichotomy with invariant projector $Q$. Then, there exists a $\beta>0$ with

$$
U_{\beta}(\mathbb{P} \mathcal{R}(Q(p))) \cap U_{\beta}(\mathbb{P} \mathcal{N}(Q(p)))=\emptyset \quad \text { for all } p \in P
$$

(i.e., $\mathbb{P} \mathcal{R}(Q)$ and $\mathbb{P} \mathcal{N}(Q)$ are all-time isolated),

$$
\lim _{t \rightarrow \infty} \sup _{p \in P} \frac{\sup _{v \in \mathbb{S}^{N-1} \cap \mathcal{N}(Q(p))}\|\Phi(-t, p) v\|}{\inf _{w \in \mathbb{S}^{N-1} \cap \mathbb{P}^{-1} U_{\beta}(\mathbb{P} \mathcal{R}(Q(p)))}\|\Phi(-t, p) w\|}=0
$$

and

$$
\lim _{t \rightarrow \infty} \sup _{p \in P} \frac{\sup _{v \in \mathbb{S}^{N-1} \cap \mathcal{R}(Q(p))}\|\Phi(t, p) v\|}{\inf _{w \in \mathbb{S}^{N-1} \cap \mathbb{P}^{-1} U_{\beta}(\mathbb{P N}(Q(p)))}\|\Phi(t, p) w\|}=0
$$

Proof. (i) Suppose that $(\theta, \varphi)$ admits a nonhyperbolic past exponential dichotomy with growth rate $\gamma$, constants $\alpha>0, K \geq 1$ and projector $Q$. We define $\beta:=\frac{1}{3 K}$, fix an arbitrary $p \in P$ and choose $\hat{p} \in[p]$ as in Definition 4.1.4 (i). The remaining proof of (i) is divided into four steps.

STEP 1. The sets $\mathbb{P} \mathcal{R}(Q)$ and $\mathbb{P N}(Q)$ are past isolated.

Assume, there exists a $t \geq 0$ such that $U_{\beta}\left(\mathbb{P} \mathcal{R}\left(Q\left(\theta_{-t} \hat{p}\right)\right)\right) \cap U_{\beta}\left(\mathbb{P} \mathcal{N}\left(Q\left(\theta_{-t} \hat{p}\right)\right)\right) \neq \emptyset$. Hence, there exist $x \in \mathbb{P} \mathcal{R}\left(Q\left(\theta_{-t} \hat{p}\right)\right)$ and $y \in \mathbb{P N}\left(Q\left(\theta_{-t} \hat{p}\right)\right)$ with $d_{\mathbb{P}}(x, y) \leq 2 \beta$. Due to the definition of $d_{\mathbb{P}}$ (cf. Appendix A.3), there exist $\tilde{x} \in \mathbb{S}^{N-1} \cap \mathbb{P}^{-1}\{x\}$ and $\tilde{y} \in \mathbb{S}^{N-1} \cap \mathbb{P}^{-1}\{y\}$ such that $\|\tilde{x}-\tilde{y}\| \leq 2 \beta$. This yields

$$
\frac{\left\|Q\left(\theta_{-t} \hat{p}\right)(\tilde{x}-\tilde{y})\right\|}{\|\tilde{x}-\tilde{y}\|}=\frac{\|\tilde{x}\|}{\|\tilde{x}-\tilde{y}\|} \geq \frac{1}{2 \beta}=\frac{3 K}{2},
$$

and this is a contradiction, since Definition 4.1 .4 (i) implies $\left\|Q\left(\theta_{-t} \hat{p}\right)\right\| \leq K$.

STEP 2. We have

$$
\left\|\Phi\left(-t, \theta_{-\tau} \hat{p}\right) x\right\| \geq \frac{1}{K} e^{-(\gamma-\alpha) t}\|x\| \quad \text { for all } \tau, t \geq 0 \text { and } x \in \mathcal{R}\left(Q\left(\theta_{-\tau} \hat{p}\right)\right) .
$$

The assertion follows from

$$
\|x\|=\left\|\Phi\left(t, \theta_{-\tau-t} \hat{p}\right) \Phi\left(-t, \theta_{-\tau} \hat{p}\right) Q\left(\theta_{-\tau} \hat{p}\right) x\right\| \stackrel{\text { Def. } 4.1 .4(\mathrm{i})}{\leq} K e^{(\gamma-\alpha) t}\left\|\Phi\left(-t, \theta_{-\tau} \hat{p}\right) x\right\| .
$$

STEP 3. Let $M \subset \mathbb{S}^{N-1} \backslash \mathcal{N}(Q(\hat{p}))$ be a compact set. For $w \in M$, we write $w=w_{r}+w_{n}$ with $w_{r} \in \mathcal{R}(Q(\hat{p}))$ and $w_{n} \in \mathcal{N}(Q(\hat{p}))$. Then,

$$
W_{r}(M):=\left\{w_{r}: w \in M\right\}=Q(\hat{p}) M
$$


is bounded away from zero, and

$$
W_{n}(M):=\left\{w_{n}: w \in M\right\}=(\mathbb{1}-Q(\hat{p})) M
$$

is bounded.

Assume, the set $W_{r}(M)=Q(\hat{p}) M$ is not bounded away from zero. Then, it contains 0 , since it is compact, and thus, there exists a $w \in M$ with $w \in \mathcal{N}(Q(\hat{p}))$. This is a contradiction. Moreover, the set $W_{n}(M)=(\mathbb{1}-Q(\hat{p})) M$ is bounded, since it is compact.

STEP 4. For all compact sets $C \subset \mathbb{S}^{N-1} \backslash \mathcal{N}(Q(p))$, we have

$$
\lim _{t \rightarrow \infty} \frac{\sup _{v \in \mathbb{S}^{N-1} \cap \mathcal{N}(Q(p))}\|\Phi(-t, p) v\|}{\inf _{w \in C}\|\Phi(-t, p) w\|}=0 .
$$

Choose $\tau \in \mathbb{T}$ such that $\hat{p}=\theta_{\tau} p$. Defining $\hat{C}:=\varphi(\tau, p) C$, it is sufficient to show

$$
\lim _{t \rightarrow \infty} \frac{\sup _{v \in \mathbb{S}^{N-1} \cap \mathcal{N}(Q(\hat{p}))}\|\Phi(-t, \hat{p}) v\|}{\inf _{w \in \hat{C}}\|\Phi(-t, \hat{p}) w\|}=0 .
$$

We have

$$
\begin{aligned}
& \frac{\sup _{v \in \mathbb{S}^{N-1} \cap \mathcal{N}(Q(\hat{p}))}\|\Phi(-t, \hat{p}) v\|}{\inf _{w \in \hat{C}}\|\Phi(-t, \hat{p}) w\|} \stackrel{\text { Def. 4.1.4 (i) }}{\leq} \frac{\sup _{v \in \mathbb{S}^{N-1} \cap \mathcal{N}(Q(\hat{p}))} K e^{-(\gamma+\alpha) t}\|v\|}{\inf _{w \in \hat{C}}\left\|\Phi(-t, \hat{p}) w_{r}+\Phi(-t, \hat{p}) w_{n}\right\|} \\
\leq & \sup _{w \in \hat{C}} \frac{\frac{K e^{-(\gamma+\alpha) t}}{\left\|\Phi(-t, \hat{p}) w_{r}\right\|}}{\left|1-\frac{\left\|\Phi(-t, \hat{p}) w_{n}\right\|}{\left\|\Phi(-t, \hat{p}) w_{r}\right\|}\right|} .
\end{aligned}
$$

Please note that for the last inequality, we require $w_{r} \neq 0$ for all $w \in \hat{C}$. This is fulfilled, since $W_{r}(\hat{C})$ is bounded away from zero (cf. Step 3). Furthermore, using

$$
\frac{K e^{-(\gamma+\alpha) t}}{\left\|\Phi(-t, \hat{p}) w_{r}\right\|} \stackrel{\text { Step } 2}{\leq} \frac{K e^{-(\gamma+\alpha) t}}{\frac{1}{K} e^{-(\gamma-\alpha) t}\left\|w_{r}\right\|}=\frac{K^{2} e^{-2 \alpha t}}{\left\|w_{r}\right\|},
$$

we obtain

$$
\lim _{t \rightarrow \infty} \sup _{w \in \hat{C}} \frac{K e^{-(\gamma+\alpha) t}}{\left\|\Phi(-t, \hat{p}) w_{r}\right\|}=0
$$

since $W_{r}(\hat{C})$ is bounded away from zero. Moreover, due to

$$
\frac{\left\|\Phi(-t, \hat{p}) w_{n}\right\|}{\left\|\Phi(-t, \hat{p}) w_{r}\right\|} \stackrel{\text { Def. 4.1.4 (i), Step } 2}{\leq} \frac{K e^{-(\gamma+\alpha) t}\left\|w_{n}\right\|}{\frac{1}{K} e^{-(\gamma-\alpha) t}\left\|w_{r}\right\|}=\frac{K^{2} e^{-2 \alpha t}\left\|w_{n}\right\|}{\left\|w_{r}\right\|},
$$

we get

$$
\lim _{t \rightarrow \infty} \sup _{w \in \hat{C}} \frac{\left\|\Phi(-t, \hat{p}) w_{n}\right\|}{\left\|\Phi(-t, \hat{p}) w_{r}\right\|}=0
$$

(please note that Step 3 says that $W_{n}(\hat{C})$ is bounded and $W_{r}(\hat{C})$ is bounded away from zero). This implies the assertion.

(ii) can be be proved similarly to (i).

(iii) Suppose that $(\theta, \varphi)$ admits a nonhyperbolic all-time exponential dichotomy with constants $\alpha>0, K \geq 1$ and projector $Q$. We define $\beta:=\frac{1}{3 K}$. The remaining proof of (iii) is divided into 
five steps.

STEP 1. The sets $\mathbb{P} \mathcal{R}(Q)$ and $\mathbb{P N}(Q)$ are all-time isolated.

Assume, there exists a $p \in P$ such that $U_{\beta}(\mathbb{P} \mathcal{R}(Q(p))) \cap U_{\beta}(\mathbb{P N}(Q(p))) \neq \emptyset$. Hence, there exist $x \in \mathbb{P} \mathcal{R}(Q(p))$ and $y \in \mathbb{P N}(Q(p))$ with $d_{\mathbb{P}}(x, y) \leq 2 \beta$. Due to the definition of $d_{\mathbb{P}}$ (cf. Appendix A.3), there exist $\tilde{x} \in \mathbb{S}^{N-1} \cap \mathbb{P}^{-1}\{x\}$ and $\tilde{y} \in \mathbb{S}^{N-1} \cap \mathbb{P}^{-1}\{y\}$ with $\|\tilde{x}-\tilde{y}\| \leq 2 \beta$. This yields

$$
\frac{\|Q(p)(\tilde{x}-\tilde{y})\|}{\|\tilde{x}-\tilde{y}\|}=\frac{\|\tilde{x}\|}{\|\tilde{x}-\tilde{y}\|} \geq \frac{1}{2 \beta}=\frac{3 K}{2} .
$$

This is a contradiction, since Definition 4.1.4 (iii) implies $\|Q(p)\| \leq K$.

STEP 2. We have

$$
\|\Phi(-t, p) x\| \geq \frac{1}{K} e^{-(\gamma-\alpha) t}\|x\| \text { for all } p \in P, t \geq 0 \text { and } x \in \mathcal{R}(Q(p)) .
$$

The assertion follows from

$$
\|x\|=\left\|\Phi\left(t, \theta_{-t} p\right) \Phi(-t, p) Q(p) x\right\| \stackrel{\text { Def. 4.1.4 (iii) }}{\leq} K e^{(\gamma-\alpha) t}\|\Phi(-t, p) x\| .
$$

STEP 3. For $p \in P$ and $w \in \mathbb{S}^{N-1} \cap \mathbb{P}^{-1} U_{\beta}(\mathbb{P} \mathcal{R}(Q(p)))$, we write $w=w_{r}^{p}+w_{n}^{p}$ with $w_{r}^{p} \in \mathcal{R}(Q(p))$ and $w_{n}^{p} \in \mathcal{N}(Q(p))$. Then,

$$
W_{r}:=\left\{w_{r}^{p}: p \in P, w \in \mathbb{S}^{N-1} \cap \mathbb{P}^{-1} U_{\beta}(\mathbb{P} \mathcal{R}(Q(p)))\right\}
$$

is bounded away from zero, and

$$
W_{n}:=\left\{w_{n}^{p}: p \in P, w \in \mathbb{S}^{N-1} \cap \mathbb{P}^{-1} U_{\beta}(\mathbb{P} \mathcal{R}(Q(p)))\right\}
$$

is bounded.

To show that $W_{r}$ is bounded away from zero, assume for contradiction, there exist sequences $\left\{p_{n}\right\}_{n \in \mathbb{N}}$ in $P$ and $\left\{w^{(n)}\right\}_{n \in \mathbb{N}}$ in $\mathbb{S}^{N-1}$ such that

$$
w^{(n)} \in \mathbb{S}^{N-1} \cap \mathbb{P}^{-1} U_{\beta}\left(\mathbb{P} \mathcal{R}\left(Q\left(p_{n}\right)\right)\right) \text { for all } n \in N
$$

and $\lim _{n \rightarrow \infty} w_{r}^{(n) p_{n}}=0$. Hence, $\lim _{n \rightarrow \infty} d_{\mathbb{P}}\left(w^{(n)}, \mathbb{P} \mathcal{N}\left(Q\left(p_{n}\right)\right)\right)=0$, and this is a contradiction to Step 1. Moreover, since

$$
\left\|w_{n}^{p}\right\|=\|(\mathbb{1}-Q(p)) w\| \stackrel{\text { Def. 4.1.4 (iii) }}{\leq} K \quad \text { for all } p \in P \text { and } w \in \mathbb{S}^{N-1} \cap \mathbb{P}^{-1} U_{\beta}(\mathbb{P} \mathcal{R}(Q(p))),
$$

the set $W_{n}$ is bounded.

STEP 4. The relation

$$
\lim _{t \rightarrow \infty} \sup _{p \in P} \frac{\sup _{v \in \mathbb{S}^{N-1} \cap \mathcal{N}(Q(p))}\|\Phi(-t, p) v\|}{\inf _{w \in \mathbb{S}^{N-1} \cap \mathbb{P}^{-1} U_{\beta}(\mathbb{P R}(Q(p)))}\|\Phi(-t, p) w\|}=0
$$

is fulfilled.

For $p \in P$, we have

$$
\begin{array}{ccc} 
& \frac{\sup _{v \in \mathbb{S}^{N-1} \cap \mathcal{N}(Q(p))}\|\Phi(-t, p) v\|}{\inf _{w \in \mathbb{S}^{N-1} \cap \mathbb{P}^{-1} U_{\beta}(\mathbb{P R}(Q(p)))}\|\Phi(-t, p) w\|} \\
\text { Def. 4.1.4 (iii) } & \frac{\sup _{v \in \mathbb{S}^{N-1} \cap \mathcal{N}(Q(p))} K e^{-(\gamma+\alpha) t}\|v\|}{\inf _{w \in \mathbb{S}^{N-1} \cap \mathbb{P}^{-1} U_{\beta}(\mathbb{P} \mathcal{R}(Q(p)))}\left\|\Phi(-t, p) w_{r}^{p}+\Phi(-t, p) w_{n}^{p}\right\|} \\
\leq & \sup _{w \in \mathbb{S}^{N-1} \cap \mathbb{P}^{-1} U_{\beta}(\mathbb{P R}(Q(p)))} \frac{\frac{K e^{-(\gamma+\alpha) t}}{\left\|\Phi(-t, p) w_{r}^{p}\right\|}}{\left|1-\frac{\left\|\Phi(-t, p) w_{n}^{p}\right\|}{\left\|\Phi(-t, p) w_{r}^{p}\right\|}\right|} .
\end{array}
$$


Please note that for the last inequality, we require $w_{r}^{p} \neq 0$ for all $w \in \mathbb{S}^{N-1} \cap \mathbb{P}^{-1} U_{\beta}(\mathbb{P} \mathcal{R}(Q(p)))$. This is fulfilled, since $W_{r}$ is bounded away from zero (cf. Step 3). Furthermore, using

$$
\frac{K e^{-(\gamma+\alpha) t}}{\left\|\Phi(-t, p) w_{r}^{p}\right\|} \stackrel{\text { Step } 2}{\leq} \frac{K e^{-(\gamma+\alpha) t}}{\frac{1}{K} e^{-(\gamma-\alpha) t}\left\|w_{r}^{p}\right\|}=\frac{K^{2} e^{-2 \alpha t}}{\left\|w_{r}^{p}\right\|},
$$

we obtain

$$
\lim _{t \rightarrow \infty} \sup _{p \in P} \sup _{w \in \mathbb{S}^{N-1} \cap \mathbb{P}^{-1} U_{\beta}(\mathbb{P} \mathcal{R}(Q(p)))} \frac{K e^{-(\gamma+\alpha) t}}{\left\|\Phi(-t, p) w_{r}^{p}\right\|}=0,
$$

since $W_{r}$ is bounded away from zero. Moreover, due to

$$
\frac{\left\|\Phi(-t, p) w_{n}^{p}\right\|}{\left\|\Phi(-t, p) w_{r}^{p}\right\|} \quad \stackrel{\text { Def. } 4.1 .4 \text { (iii), Step } 2}{\leq} \frac{K e^{-(\gamma+\alpha) t}\left\|w_{n}^{p}\right\|}{\frac{1}{K} e^{-(\gamma-\alpha) t}\left\|w_{r}^{p}\right\|}=\frac{K^{2} e^{-2 \alpha t}\left\|w_{n}^{p}\right\|}{\left\|w_{r}^{p}\right\|}
$$

we get

$$
\lim _{t \rightarrow \infty} \sup _{p \in P} \sup _{w \in \mathbb{S}^{N-1} \cap \mathbb{P}^{-1} U_{\beta}(\mathbb{P R}(Q(p)))} \frac{\left\|\Phi(-t, p) w_{n}^{p}\right\|}{\left\|\Phi(-t, p) w_{r}^{p}\right\|}=0
$$

(please note that Step 3 says that $W_{n}$ is bounded and $W_{r}$ is bounded away from zero). This implies the assertion.

STEP 5. The relation

$$
\lim _{t \rightarrow \infty} \sup _{p \in P} \frac{\sup _{v \in \mathbb{S}^{N-1} \cap \mathcal{R}(Q(p))}\|\Phi(t, p) v\|}{\inf _{w \in \mathbb{S}^{N-1} \cap \mathbb{P}^{-1} U_{\beta}(\mathbb{P N}(Q(p)))}\|\Phi(t, p) w\|}=0
$$

is fulfilled.

See proof of Step 4.

The following theorem says that ranges and null spaces of invariant projectors give rise to nonautonomous repellers and attractors. Similar questions are treated in PALMER \& SIEGMUND [127, Proposition 3.1], where so-called generalized attractor-repeller pairs on the projective space are examined.

4.1.15 THEOREM (RANGes AND Null SpaCES OF INVARIANT PROJECTORS AS NONAUTONOMOUS REPELLERS AND ATTRACTORS). We suppose that $(\theta, \varphi)$ admits a nonhyperbolic past (future, all-time, respectively) exponential dichotomy with projector $Q$ and consider the nonautonomous dynamical system $(\theta, \mathbb{P} \Phi)$ on the real projective space $\mathbb{P}^{N-1}$. Then, the following statements are fulfilled:

(i) $\mathbb{P} \mathcal{R}(Q)$ is a past (future, all-time, respectively) repeller,

(ii) $\mathbb{P N}(Q)$ is a past (future, all-time, respectively) attractor,

(iii) in case of a nonhyperbolic past exponential dichotomy, we have $\mathbb{P} \mathcal{N}(Q)=\mathbb{P} \mathcal{R}(Q)^{*}$, and in case of a nonhyperbolic future exponential dichotomy, $\mathbb{P} \mathcal{R}(Q)=\mathbb{P N}(Q)^{*}$ is fulfilled.

PROOF. In case of a nonhyperbolic past exponential dichotomy, the fact that $\operatorname{PR}(Q)$ is a past repeller can be proved as in Proposition 3.5.2 (Step 1 to Step 4), where instead of Proposition 3.5.1 and Step 3 one should use Lemma 4.1.14 (i). Moreover, the proof that $\mathbb{P} \mathcal{N}(Q)$ is a past attractor and $\mathbb{P} \mathcal{N}(Q)=\mathbb{P} \mathcal{R}(Q)^{*}$ is analogous to Step 5 of the proof of Proposition 3.5.2. 
The assertions concerning the case of a nonhyperbolic future exponential dichotomy are now easily obtained by using Proposition 2.1.22.

In case $(\theta, \varphi)$ admits a nonhyperbolic all-time exponential dichotomy, we now prove that $\mathbb{P} \mathcal{R}(Q)$ is an all-time repeller. First, we choose $\beta>0$ from Lemma 4.1 .14 (iii). The remaining proof is divided into two steps.

STEP 1. We have

$$
\begin{aligned}
& 1=\lim _{t \rightarrow \infty} \inf _{p \in P} \inf _{0 \neq v \in \mathbb{P}^{-1}} U_{\beta}(\mathbb{P R}(Q(p))) \quad \frac{\left\|\Phi(-t, p) v_{r}^{p}\right\|}{\|\Phi(-t, p) v\|} \\
& =\lim _{t \rightarrow \infty} \sup _{p \in P} \sup _{0 \neq v \in \mathbb{P}^{-1} U_{\beta}(\mathbb{P R}(Q(p)))} \frac{\left\|\Phi(-t, p) v_{r}^{p}\right\|}{\|\Phi(-t, p) v\|},
\end{aligned}
$$

where $v=v_{r}^{p}+v_{n}^{p}$ with $v_{r}^{p} \in \mathcal{R}(Q(p))$ and $v_{n}^{p} \in \mathcal{N}(Q(p))$.

The first assertion follows from

$$
\begin{aligned}
& \lim _{t \rightarrow \infty} \inf _{p \in P} \inf _{0 \neq v \in \mathbb{P}^{-1} U_{\beta}(\mathbb{P R}(Q(p)))} \frac{\left\|\Phi(-t, p) v_{r}^{p}\right\|}{\|\Phi(-t, p) v\|} \\
& \geq \quad\left(\lim _{t \rightarrow \infty} \sup _{p \in P} \sup _{0 \neq v \in \mathbb{P}^{-1} U_{\beta}(\mathbb{P R}(Q(p)))} \frac{\left\|\Phi(-t, p) v_{n}^{p}\right\|}{\left\|\Phi(-t, p) v_{r}^{p}\right\|}+1\right)^{-1} \\
& =\left(\lim _{t \rightarrow \infty} \sup _{p \in P} \sup _{v \in \mathbb{P}^{-1} U_{\beta}(\mathbb{P} \mathcal{R}(Q(p))), v_{n}^{p} \neq 0} \frac{\left\|v_{n}^{p}\right\|\left\|\Phi(-t, p) \frac{v_{n}^{p}}{\left\|v_{n}^{p}\right\|}\right\|}{\left\|v_{r}^{p}\right\|\left\|\Phi(-t, p) \frac{v_{r}^{p}}{\left\|v_{r}^{p}\right\|}\right\|}+1\right)^{-1}
\end{aligned}
$$

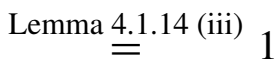

and

$$
\begin{aligned}
& \lim _{t \rightarrow \infty} \inf _{p \in P} \inf _{0 \neq v \in \mathbb{P}^{-1} U_{\beta}(\mathbb{P} \mathcal{R}(Q(p)))} \frac{\left\|\Phi(-t, p) v_{r}^{p}\right\|}{\|\Phi(-t, p) v\|} \\
& \leq \quad\left(\lim _{t \rightarrow \infty} \sup _{p \in P} \sup _{0 \neq v \in \mathbb{P}^{-1} U_{\beta}(\mathbb{P R}(Q(p)))}\left|1-\frac{\left\|\Phi(-t, p) v_{n}^{p}\right\|}{\left\|\Phi(-t, p) v_{r}^{p}\right\|}\right|\right)^{-1} \\
& =\left(\lim _{t \rightarrow \infty} \sup _{p \in P} \sup _{v \in \mathbb{P}^{-1} U_{\beta}(\mathbb{P} \mathcal{R}(Q(p))), v_{n}^{p} \neq 0} \mid 1-\frac{\left\|v_{n}^{p}\right\|\left\|\Phi(-t, p) \frac{v_{n}^{p}}{\left\|v_{n}^{p}\right\|}\right\|}{\left\|v_{r}^{p}\right\|\left\|\Phi(-t, p) \frac{v_{r}^{p}}{\left\|v_{r}^{p}\right\|}\right\|} \|\right)^{-1}
\end{aligned}
$$

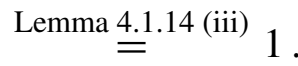

In both relations, the last equality holds, because the sets

$$
\left\{v_{n}^{p}: v \in \mathbb{P}^{-1} U_{\beta}(\mathbb{P} \mathcal{R}(Q(p)))\right\} \quad \text { for all } p \in P
$$

are compact and the sets

$$
\left\{v_{r}^{p}: v \in \mathbb{P}^{-1} U_{\beta}(\mathbb{P} \mathcal{R}(Q(p)))\right\} \quad \text { for all } p \in P
$$


are bounded away from zero (cf. also Step 3 of Lemma 4.1.14 and Step 1 of Proposition 3.5.2). The assertion

$$
1=\lim _{t \rightarrow \infty} \sup _{p \in P} \sup _{0 \neq v \in \mathbb{P}^{-1} U_{\beta}(\mathbb{P} \mathcal{R}(Q(p)))} \frac{\left\|\Phi(-t, p) v_{r}^{p}\right\|}{\|\Phi(-t, p) v\|},
$$

follows analogously.

STEP 2. We have

$$
\lim _{t \rightarrow \infty} \sup _{p \in P} d_{\mathbb{P}}\left(\mathbb{P} \Phi(-t, p) U_{\beta}(\mathbb{P} \mathcal{R}(Q(p))) \mid \mathbb{P} \mathcal{R}\left(Q\left(\theta_{-t} p\right)\right)\right)=0
$$

i.e., $\operatorname{PR}(Q(p))$ is an all-time repeller.

With $v_{r}^{p}$ and $v_{n}^{p}$ defined as in Step 1 , for all $t \geq 0, p \in P$ and $v \in \mathbb{S}^{N-1} \cap \mathbb{P}^{-1} U_{\beta}(\mathbb{P} \mathcal{R}(Q(p)))$, the relation

$$
\begin{aligned}
& \frac{\left\langle\Phi(-t, p) v, \Phi(-t, p) v_{r}^{p}\right\rangle^{2}}{\|\Phi(-t, p) v\|^{2}\left\|\Phi(-t, p) v_{r}^{p}\right\|^{2}} \\
= & \frac{\left\langle\Phi(-t, p) v_{n}^{p}, \Phi(-t, p) v_{r}^{p}\right\rangle^{2}}{\|\Phi(-t, p) v\|^{2}\left\|\Phi(-t, p) v_{r}^{p}\right\|^{2}}+\frac{\left\|\Phi(-t, p) v_{r}^{p}\right\|^{2}}{\|\Phi(-t, p) v\|^{2}}+\frac{2\left\langle\Phi(-t, p) v_{n}^{p}, \Phi(-t, p) v_{r}^{p}\right\rangle}{\|\Phi(-t, p) v\|^{2}}
\end{aligned}
$$

holds (cf. Step 2 of the proof of Proposition 3.5.2). Using the Cauchy-Schwartz inequality, we obtain the following relations:

$$
\begin{aligned}
& 0 \leq \lim _{t \rightarrow \infty} \sup _{p \in P} \sup _{v \in \mathbb{S}^{N-1} \cap \mathbb{P}^{-1} U_{\beta}(\mathbb{P R}(Q(p)))} \frac{\left\langle\Phi(-t, p) v_{n}^{p}, \Phi(-t, p) v_{r}^{p}\right\rangle^{2}}{\|\Phi(-t, p) v\|^{2}\left\|\Phi(-t, p) v_{r}^{p}\right\|^{2}} \leq \\
& \lim _{t \rightarrow \infty} \sup _{p \in P} \sup _{v \in \mathbb{S}^{N-1} \cap \mathbb{P}^{-1} U_{\beta}(\mathbb{P R}(Q(p)))} \frac{\left\|\Phi(-t, p) v_{n}^{p}\right\|^{2}}{\|\Phi(-t, p) v\|^{2}} \stackrel{\text { Lemma 4.1.14 (iii) }}{=} 0
\end{aligned}
$$

and

$$
\begin{aligned}
& 0 \leq \lim _{t \rightarrow \infty} \sup _{p \in P} \sup _{v \in \mathbb{S}^{N-1} \cap \mathbb{P}^{-1} U_{\beta}(\mathbb{P R}(Q(p)))} \frac{2\left|\left\langle\Phi(-t, p) v_{n}^{p}, \Phi(-t, p) v_{r}^{p}\right\rangle\right|}{\|\Phi(-t, p) v\|^{2}} \\
& \leq \quad \lim _{t \rightarrow \infty} \sup _{p \in P} \sup _{v \in \mathbb{S}^{N-1} \cap \mathbb{P}^{-1} U_{\beta}(\mathbb{P R}(Q(p)))} 2 \frac{\left\|\Phi(-t, p) v_{n}^{p}\right\|}{\|\Phi(-t, p) v\|} \frac{\left\|\Phi(-t, p) v_{r}^{p}\right\|}{\|\Phi(-t, p) v\|} \\
& \stackrel{\text { Step } 1}{=} \quad \lim _{t \rightarrow \infty} \sup _{p \in P} \sup _{v \in \mathbb{S}^{N-1} \cap \mathbb{P}^{-1} U_{\beta}(\mathbb{P R}(Q(p)))} \frac{2\left\|\Phi(-t, p) v_{n}^{p}\right\|}{\|\Phi(-t, p) v\|}
\end{aligned}
$$

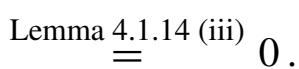

Hence, due to Step 1, we have

$$
\lim _{t \rightarrow \infty} \inf _{p \in P} \inf _{v \in \mathbb{S}^{N-1} \cap \mathbb{P}^{-1} U_{\beta}(\mathbb{P R}(Q(p)))} \frac{\left\langle\Phi(-t, p) v, \Phi(-t, p) v_{r}^{p}\right\rangle^{2}}{\|\Phi(-t, p) v\|^{2}\left\|\Phi(-t, p) v_{r}^{p}\right\|^{2}}=
$$




$$
\begin{gathered}
=\lim _{t \rightarrow \infty} \inf _{p \in P} \inf _{v \in \mathbb{S}^{N-1} \cap \mathbb{P}^{-1} U_{\beta}(\mathbb{P R}(Q(p)))}\left(\frac{\left\langle\Phi(-t, p) v_{n}^{p}, \Phi(-t, p) v_{r}^{p}\right\rangle^{2}}{\|\Phi(-t, p) v\|^{2}\left\|\Phi(-t, p) v_{r}^{p}\right\|^{2}}+\frac{\left\|\Phi(-t, p) v_{r}^{p}\right\|^{2}}{\|\Phi(-t, p) v\|^{2}}\right. \\
\left.+\frac{2\left\langle\Phi(-t, p) v_{n}^{p}, \Phi(-t, p) v_{r}^{p}\right\rangle}{\|\Phi(-t, p) v\|^{2}}\right)
\end{gathered}
$$

$=1$.

Using Lemma A.3.1, this implies that $\mathbb{P} \mathcal{R}(Q)$ is an all-time repeller.

Moreover, with Proposition 2.1.22, it is easy to show that $\mathbb{P} \mathcal{N}(Q)$ is an all-time attractor. This finishes the proof of this theorem.

\subsection{Dichotomy SPECTRA}

In the previous section, notions of dichotomy have been introduced by localizing attractive and repulsive directions. To classify the strength of attractivity and repulsivity of linear systems, the concept of the dichotomy spectrum is essential. For linear skew product flows with compact base sets, the so-called Sacker-Sell spectrum (see SACKER \& SELL [153]) has become widely accepted. In Siegmund [171] and AulBaCH \& Siegmund [19], this spectrum has been adapted for arbitrary classes of linear differential and difference equations, respectively (for the noninvertible case, see AULBACH \& SIEGMUND [20]). In addition to this dichotomy spectrum, in this section, three other kinds of spectra are introduced with respect to the notions of past, future and finite-time attractivity and repulsivity. Thereby, attention is restricted to the following situation.

STANDING HYPOTHESIS. We suppose that $(\theta, \varphi)$ is generated by the nonautonomous differential equation

$$
\dot{x}=A(t) x \text {, }
$$

$P=\mathbb{T}=\mathbb{R}$, or the nonautonomous difference equation

$$
x_{n+1}=A(n) x_{n} \text {, }
$$

$P=\mathbb{T}=\mathbb{Z}$, where $A: \mathbb{T} \rightarrow \mathbb{R}^{N \times N}$ is a continuous function. The base flow fulfills the relation $\theta(t, \tau)=t+\tau$ for all $t, \tau \in \mathbb{T}$ (cf. Section 1.2).

This restriction is necessary, since we do not want to make assumptions concerning the structure of the base flow $\theta$ and the base set $P$ such as compactness, minimality, chain recurrence or invariant connectedness.

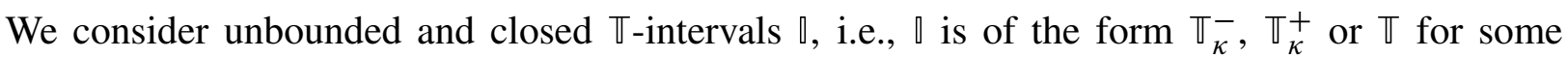

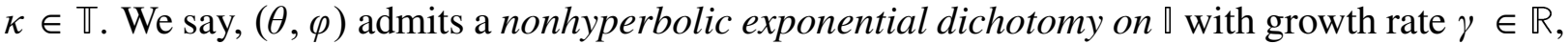
constants $\alpha>0, K \geq 1$ and invariant projector $Q$ if

$$
\begin{array}{rlrl}
\|\Phi(t, \tau) Q(\tau)\| & \leq K e^{(\gamma-\alpha) t} & & \text { for all } \tau \in \mathbb{Q}, t \geq 0 \text { with } \tau+t \in \mathbb{}, \\
\|\Phi(-t, \tau)(\mathbb{1}-Q(\tau))\| & \leq K e^{-(\gamma+\alpha) t} & \text { for all } \tau \in \mathbb{Q}, t \geq 0 \text { with } \tau-t \in \mathbb{}
\end{array}
$$

The following proposition says that this definition coincides with the notions of nonhyperbolic exponential dichotomy from in the previous section. 
4.2.1 Proposition. Let $\kappa \in \mathbb{T}$ and $\gamma \in \mathbb{R}$. Then, the following statements are fulfilled:

(i) $(\theta, \varphi)$ admits a nonhyperbolic exponential dichotomy on $\mathbb{T}_{\kappa}^{-}$with growth rate $\gamma$ if and only if $(\theta, \varphi)$ admits a nonhyperbolic past exponential dichotomy with growth rate $\gamma$,

(ii) $(\theta, \varphi)$ admits a nonhyperbolic exponential dichotomy on $\mathbb{\mathbb { T }}_{\kappa}^{+}$with growth rate $\gamma$ if and only if $(\theta, \varphi)$ admits a nonhyperbolic future exponential dichotomy with growth rate $\gamma$,

(iii) $(\theta, \varphi)$ admits a nonhyperbolic exponential dichotomy on $\mathbb{T}$ with growth rate $\gamma$ if and only if $(\theta, \varphi)$ admits a nonhyperbolic all-time exponential dichotomy with growth rate $\gamma$.

Proof. (i) $(\Rightarrow$ ) The conditions of Proposition 4.1 .10 (i) are fulfilled by choosing $\hat{p}:=\kappa$.

$(\Leftarrow)$ Suppose, $(\theta, \varphi)$ admits a nonhyperbolic past exponential dichotomy with growth rate $\gamma$, constants $\alpha, K$ and projector $Q$. Thus, there exists a $\hat{p} \in \mathbb{T}$ such that the two conditions in Proposition 4.1 .10 (i) are fulfilled. In case $\hat{p} \geq \kappa$, the assertion follows immediately. Otherwise, we define

$$
\begin{aligned}
\tilde{K}:=\max \{ & \max \{\|\Phi(t, \tau) Q(\tau)\|: t, \tau \in[\hat{p}, \kappa] \cap \mathbb{T} \text { with } t \geq \tau\}, \\
& \max \{\|\Phi(t, \tau)(\mathbb{1}-Q(\tau))\|: t, \tau \in[\hat{p}, \kappa] \cap \mathbb{T} \text { with } t \leq \tau\}\} .
\end{aligned}
$$

Then, $(\theta, \varphi)$ admits a nonhyperbolic exponential dichotomy on $\mathbb{T}_{\kappa}^{-}$with growth rate $\gamma$ and constants $\alpha, K \tilde{K}$.

(ii) can be shown analogously to (i), and (iii) is obviously fulfilled.

In this section, we make use of these alternative characterizations instead of Definition 4.1.7.

4.2.2 REMARK. In case the function $A$ of the differential equation (4.2) or difference equation (4.3) is only defined on an interval of the form $\mathbb{T}_{\kappa}^{-}$or $\mathbb{T}_{\kappa}^{+}$for some $\kappa \in \mathbb{T}$, respectively, the nonautonomous dynamical system generated by this equation does not fulfill the hypotheses of this chapter. Due to Proposition 4.2.1, however, we are able to use the notions of past or future exponential dichotomy and the notions of past and future dichotomy spectrum (see Definition 4.2.5 below) also for these types of equations.

Given an invariant projector $Q$, the fibres of $\mathcal{R}(Q)$ and $\mathcal{N}(Q)$, respectively, have the same dimension, since the base set $P$ is a trajectory of base flow $\theta$. We therefore define the rank of $Q$ by

$$
\text { rk } Q:=\operatorname{dim} \mathcal{R}(Q):=\operatorname{dim} \mathcal{R}(Q(t)) \text { for all } t \in \mathbb{T},
$$

and we set

$$
\operatorname{dim} \mathcal{N}(Q):=\operatorname{dim} \mathcal{N}(Q(t)) \text { for all } t \in \mathbb{T}
$$

4.2.3 Proposition. Suppose, both $Q$ and $\hat{Q}$ are invariant projectors of a nonhyperbolic past exponential (future exponential, all-time exponential, $(\tau, T)$-, respectively) dichotomy with growth rate $\gamma$. Then, $\operatorname{rk} Q=\operatorname{rk} \hat{Q}$ is fulfilled.

PROOF. In case of a nonhyperbolic past exponential (future exponential, all-time exponential, respectively) dichotomy, the assertion follows directly from Proposition 4.1.11. Arguing negatively, 
we suppose that $(\theta, \varphi)$ admits a $(\tau, T)$-dichotomy with two invariant projectors $Q$ and $\hat{Q}$ such that $\operatorname{rk} Q<\operatorname{rk} \hat{Q}$. Thus,

$$
\begin{aligned}
\operatorname{dim}(\mathcal{N}(Q) \cap \mathcal{R}(\hat{Q})) & =\operatorname{dim} \mathcal{N}(Q)+\operatorname{dim} \mathcal{R}(\hat{Q})-\operatorname{dim}(\mathcal{N}(Q)+\mathcal{R}(\hat{Q})) \\
& >\operatorname{dim} \mathcal{N}(Q)+\operatorname{dim} \mathcal{R}(Q)-\operatorname{dim}(\mathcal{N}(Q)+\mathcal{R}(\hat{Q})) \geq 0
\end{aligned}
$$

Hence, there exists a nonzero element $\xi \in \mathcal{N}(Q(\tau)) \cap \mathcal{R}(\hat{Q}(\tau))$. We obtain

$$
\|\Phi(T, \tau) \xi\|<\|\xi\|=\|\Phi(-T, \tau+T) \Phi(T, \tau) \xi\|<\|\Phi(T, \tau) \xi\|
$$

since $0 \neq \xi \in \mathcal{R}(\hat{Q}(\tau))$ and $0 \neq \Phi(T, \tau) \xi \in \mathcal{N}(Q(\tau+T))$. This contradiction finishes the proof of this proposition.

As indicated in Remark 4.1.12, an invariant projector is uniquely determined only in case of a nonhyperbolic all-time exponential dichotomy. The degree of nonuniqueness of projectors of past and future exponential dichotomies is described in the following lemma, which is adapted from AulbaCH \& SiEgmund [20, Lemma 2.4].

4.2.4 LEMMA. The following statements are fulfilled:

(i) We assume that $(\theta, \varphi)$ admits a nonhyperbolic past exponential dichotomy with growth rate $\gamma$ and projector $Q$, and $\hat{Q}$ is another invariant projector with

$$
\sup _{t \in \mathbb{T}_{0}^{-}}\|\hat{Q}(t)\|<\infty \quad \text { and } \quad \mathcal{N}(Q)=\mathcal{N}(\hat{Q}) .
$$

Then, $(\theta, \varphi)$ also admits a nonhyperbolic past exponential dichotomy with growth rate $\gamma$ and projector $\hat{Q}$.

(ii) We assume that $(\theta, \varphi)$ admits a nonhyperbolic future exponential dichotomy with growth rate $\gamma$ and projector $Q$, and $\hat{Q}$ is another invariant projector with

$$
\sup _{t \in \mathbb{T}_{0}^{+}}\|\hat{Q}(t)\|<\infty \quad \text { and } \quad \mathcal{R}(Q)=\mathcal{R}(\hat{Q}) .
$$

Then, $(\theta, \varphi)$ also admits a nonhyperbolic future exponential dichotomy with growth rate $\gamma$ and projector $\hat{Q}$.

Proof. (i) Suppose, $(\theta, \varphi)$ admits a nonhyperbolic exponential dichotomy on $\mathbb{T}_{0}^{-}$with growth rate $\gamma$, constants $\alpha>0, K \geq 1$ and projector $Q$, and let $\hat{Q}$ be given as above. First, we observe that $\sup _{t \in \mathbb{T}_{0}^{-}}\|Q(t)\| \leq K$, and we define $M:=\sup _{t \in \mathbb{T}_{0}^{-}}\|\hat{Q}(t)\|$. The relation $\mathcal{N}(Q)=\mathcal{N}(\hat{Q})$ implies the two equations

$$
(\mathbb{1}-\hat{Q})=(\mathbb{1}-Q)(\mathbb{1}-\hat{Q}) \quad \text { and } \quad \hat{Q}=(\mathbb{1}-Q+\hat{Q}) Q .
$$

The first equation yields for all $\tau \in \mathbb{T}_{0}^{-}$and $t \geq 0$

$$
\begin{aligned}
\|\Phi(-t, \tau)(\mathbb{1}-\hat{Q}(\tau))\| & =\|\Phi(-t, \tau)(\mathbb{1}-Q(\tau))(\mathbb{1}-\hat{Q}(\tau))\| \\
& \leq\|\Phi(-t, \tau)(\mathbb{1}-Q(\tau))\|\|\mathbb{1}-\hat{Q}(\tau)\| \leq K(1+M) e^{-(\gamma+\alpha) t} .
\end{aligned}
$$


Using the invariance of $Q$ and $\hat{Q}$, the second equation implies

$$
\begin{aligned}
\|\Phi(t, \tau) \hat{Q}(\tau)\| & =\|\Phi(t, \tau)(\mathbb{1}-Q(\tau)+\hat{Q}(\tau)) Q(\tau)\| \\
& \leq\|(\mathbb{1}-Q(\tau+t)+\hat{Q}(\tau+t))\|\|\Phi(t, \tau) Q(\tau)\| \\
& \leq K(1+K+M) e^{(\gamma-\alpha) t}
\end{aligned}
$$

for all $\tau \in \mathbb{T}_{0}^{-}$and $t \geq 0$ with $\tau+t \in \mathbb{T}_{0}^{-}$.

The assertion (ii) can be proved similarly.

It is crucial for the definition of the dichotomy spectra, for which growth rates, the linear NDS $(\theta, \varphi)$ admits a nonhyperbolic dichotomy. In case of a past exponential (future exponential, alltime exponential, respectively) dichotomy, we will not exclude growth rates $\gamma= \pm \infty$ from our considerations. We say, $(\theta, \varphi)$ admits a nonhyperbolic dichotomy with growth rate $\infty$ if there exists a $\gamma \in \mathbb{R}$ such that $(\theta, \varphi)$ admits a nonhyperbolic dichotomy with growth rate $\gamma$ and projector $P_{\gamma} \equiv \mathbb{1}$. Accordingly, we say that $(\theta, \varphi)$ admits a nonhyperbolic dichotomy with growth rate $-\infty$ if there exists a $\gamma \in \mathbb{R}$ such that $(\theta, \varphi)$ admits a nonhyperbolic dichotomy with growth rate $\gamma$ and projector $P_{\gamma} \equiv \mathbb{O}$.

\subsubsection{Definition (Dichotomy SPECTRA).}

(i) The past dichotomy spectrum of $(\theta, \varphi)$ is defined by

$$
\begin{gathered}
\Sigma_{\Phi}^{\leftarrow}:=\{\gamma \in \overline{\mathbb{R}}:(\theta, \varphi) \text { does not admit a nonhyperbolic past exponential } \\
\text { dichotomy with growth rate } \gamma\} .
\end{gathered}
$$

(ii) The future dichotomy spectrum of $(\theta, \varphi)$ is defined by

$$
\begin{gathered}
\Sigma_{\Phi}:=\{\gamma \in \bar{R}:(\theta, \varphi) \text { does not admit a nonhyperbolic future exponential } \\
\text { dichotomy with growth rate } \gamma\} .
\end{gathered}
$$

(iii) The all-time dichotomy spectrum of $(\theta, \varphi)$ is defined by

$$
\begin{aligned}
\Sigma_{\Phi}^{\leftrightarrow}:=\{\gamma \in \bar{R}: & (\theta, \varphi) \text { does not admit a nonhyperbolic all-time exponential } \\
& \text { dichotomy with growth rate } \gamma\} .
\end{aligned}
$$

(iv) Given $\tau \in \mathbb{T}$ and $T \in \mathbb{T}^{+}$, the $(\tau, T)$-dichotomy spectrum of $(\theta, \varphi)$ is defined by

$$
\begin{gathered}
\Sigma_{\Phi}^{(\tau, T)}:=\{\gamma \in \mathbb{R}:(\theta, \varphi) \text { does not admit a nonhyperbolic }(\tau, T) \text {-dichotomy } \\
\text { with growth rate } \gamma\} .
\end{gathered}
$$

The corresponding resolvent sets are defined as follows:

$$
\begin{aligned}
& \rho_{\Phi}^{\leftarrow}:=\overline{\mathbb{R}} \backslash \Sigma_{\Phi}^{\leftarrow}, \quad \quad \rho_{\Phi}:=\overline{\mathbb{R}} \backslash \Sigma_{\Phi}, \\
& \rho_{\Phi}^{\leftrightarrow}:=\overline{\mathbb{R}} \backslash \Sigma_{\Phi}^{\leftrightarrow} \quad \text { and } \quad \rho_{\Phi}^{(\tau, T)}:=\overline{\mathbb{R}} \backslash \Sigma_{\Phi}^{(p, T)} \text {. }
\end{aligned}
$$

In regard to the STANDING HYPOTHESIS, also the notation $\Sigma_{A}$ and $\rho_{A}$ is used for the dichotomy spectra and resolvent sets of $\dot{x}=A(t) x$ and $x_{n+1}=A(n) x_{n}$, respectively. 


\subsubsection{REMARKS.}

(i) The all-time dichotomy spectrum without $\{-\infty, \infty\}$, i.e., $\Sigma_{\Phi}^{\leftrightarrow} \cap \mathbb{R}$, coincides with the dichotomy spectrum for differential equations introduced in SIEGMUND [171] (see also SIEGMUND [170]). In case of linear difference equations, $\exp \left(\Sigma_{\Phi}^{\leftrightarrow} \cap \mathbb{R}\right)$ is the dichotomy spectrum introduced in AUlBACH \& SiEgmund [19].

(ii) In contrast to the past, future or all-time dichotomy spectrum, the notion of $(\tau, T)$ dichotomy spectrum is not invariant with respect to a change of the norm to an equivalent norm (cf. also Remark 4.1.5 (iii) and Remark 2.1.10).

(iii) From Proposition 4.1.6, we obtain directly $\Sigma_{\Phi}^{\leftarrow} \subset \Sigma_{\Phi}^{\leftrightarrow}$ and $\Sigma_{\Phi} \subset \Sigma_{\Phi}^{\leftrightarrow}$.

The aim of the following lemma is to analyze the topological structure of the resolvent sets.

4.2.7 LEMMA. We suppose that $\rho_{\Phi}:=\rho_{\Phi}^{\leftarrow}, \rho_{\Phi}, \rho_{\Phi}^{\leftrightarrow}, \rho_{\Phi}^{(\tau, T)}$, respectively. Then, $\rho_{\Phi} \cap \mathbb{R}$ is open, more precisely, for all $\gamma \in \rho_{\Phi} \cap \mathbb{R}$, there exists an $\varepsilon>0$ such that $U_{\varepsilon}(\gamma) \subset \rho_{\Phi}$. Furthermore, the relation $\operatorname{rk} Q_{\zeta}=\operatorname{rk} Q_{\gamma}$ is fulfilled for all $\zeta \in U_{\varepsilon}(\gamma)$ and every invariant projector $Q_{\gamma}$ and $Q_{\zeta}$ of the nonhyperbolic dichotomies of $(\theta, \varphi)$ with growth rates $\gamma$ and $\zeta$, respectively.

Proof. We first treat the case $\rho_{\Phi}=\rho_{\Phi}^{\leftarrow}, \rho_{\Phi}, \rho_{\Phi}^{\leftrightarrow}$ and choose $\gamma \in \rho_{\Phi}$ arbitrarily. Since $(\theta, \varphi)$ admits a nonhyperbolic exponential dichotomy on $\mathbb{\square}=\mathbb{\mathbb { T }}_{0}^{-}, \mathbb{\mathbb { T }}_{0}^{+}, \mathbb{\mathbb { T }}$ with growth rate $\gamma$, respectively, there exists an invariant projector $Q_{\gamma}$ and constants $\alpha>0, K \geq 1$ such that

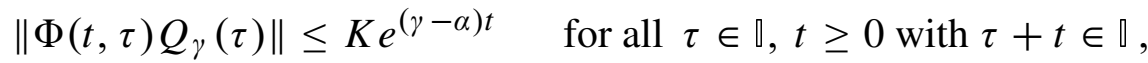

$$
\begin{aligned}
& \left\|\Phi(-t, \tau)\left(\mathbb{1}-Q_{\gamma}(\tau)\right)\right\| \leq K e^{-(\gamma+\alpha) t} \quad \text { for all } \tau \in \mathbb{\square}, t \geq 0 \text { with } \tau-t \in \mathbb{\square} \text {. }
\end{aligned}
$$

We set $\varepsilon:=\frac{\alpha}{2}$ and choose $\zeta \in U_{\varepsilon}(\gamma)$. Thus,

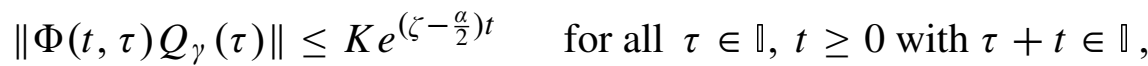

$$
\begin{aligned}
& \left\|\Phi(-t, \tau)\left(\mathbb{1}-Q_{\gamma}(\tau)\right)\right\| \leq K e^{-\left(\zeta+\frac{\alpha}{2}\right) t} \quad \text { for all } \tau \in \mathbb{Q}, t \geq 0 \text { with } \tau-t \in \mathbb{} \text {. }
\end{aligned}
$$

This yields $\zeta \in \rho_{\Phi}$. Since Proposition 4.2.3 says that the ranks of the projectors of nonhyperbolic exponential dichotomies on \ with the same growth rate are equal, we have rk $Q_{\zeta}=\operatorname{rk} Q_{\gamma}$ for any projector $Q_{\zeta}$ of the nonhyperbolic exponential dichotomy on \& with growth rate $\zeta$. In case $\rho_{\Phi}=\rho_{\Phi}^{(\tau, T)}$ and $\gamma \in \rho_{\Phi}$, there exists an invariant projector $Q_{\gamma}$ such that

$$
\begin{array}{ccc}
\|\Phi(T, \tau) \xi\|<e^{\gamma T}\|\xi\| & & \text { for all } 0 \neq \xi \in \mathcal{R}\left(Q_{\gamma}(\tau)\right), \\
\|\Phi(-T, \tau+T) \xi\|<e^{-\gamma T}\|\xi\| & \text { for all } 0 \neq \xi \in \mathcal{N}\left(Q_{\gamma}(\tau+T)\right) .
\end{array}
$$

We define

$$
\beta:=\max \left\{\max _{0 \neq \xi \in \mathcal{R}\left(Q_{\gamma}(\tau)\right)} \frac{\|\Phi(T, \tau) \xi\|}{e^{\gamma T}\|\xi\|}, \max _{0 \neq \xi \in \mathcal{N}\left(Q_{\gamma}(\tau+T)\right)} \frac{\|\Phi(T, \tau) \xi\|}{e^{\gamma T}\|\xi\|}\right\}<1
$$

and set $\varepsilon:=\frac{1}{2 T} \ln \beta$. Thus, for all $\zeta \in U_{\varepsilon}(\gamma)$, we have

$$
\begin{aligned}
\|\Phi(T, \tau) \xi\| & <e^{\zeta T}\|\xi\| & & \text { for all } 0 \neq \xi \in \mathcal{R}\left(Q_{\gamma}(\tau)\right), \\
\|\Phi(-T, \tau+T) \xi\| & <e^{-\zeta T}\|\xi\| & & \text { for all } 0 \neq \xi \in \mathcal{N}\left(Q_{\gamma}(\tau+T)\right) .
\end{aligned}
$$


This implies $\zeta \in \rho_{\Phi}$. The equality of the ranks of the invariant projectors follows from Proposition 4.2.3.

4.2.8 LemmA. Assume that $\rho_{\Phi}:=\rho_{\Phi}^{\leftarrow}, \rho_{\Phi}, \rho_{\Phi}^{\leftrightarrow}, \rho_{\Phi}^{(\tau, T)}$, respectively, let $\gamma_{1}, \gamma_{2} \in \rho_{\Phi} \cap \mathbb{R}$ with $\gamma_{1}<\gamma_{2}$, and choose invariant projectors $Q_{\gamma_{1}}$ and $Q_{\gamma_{2}}$ for the corresponding nonhyperbolic dichotomies with growth rates $\gamma_{1}$ and $\gamma_{2}$. Then, the relation $\operatorname{rk} Q_{\gamma_{1}} \leq \operatorname{rk} Q_{\gamma_{2}}$ holds. Moreover, $\left[\gamma_{1}, \gamma_{2}\right] \subset \rho_{\Phi}$ is fulfilled if and only if $\operatorname{rk} Q_{\gamma_{1}}=\operatorname{rk} Q_{\gamma_{2}}$.

PROOF. We first prove the relation $\operatorname{rk} Q_{\gamma_{1}} \leq \operatorname{rk} Q_{\gamma_{2}}$. In case $\rho_{\Phi}=\rho_{\Phi}^{\overleftarrow{ }}, \rho_{\Phi}, \rho_{\Phi}^{\leftrightarrow}$, respectively, this is a direct consequence of Proposition 4.1.11, since $\mathcal{S}_{\gamma_{1}} \subset \mathcal{S}_{\gamma_{2}}$ and $\mathcal{U}_{\gamma_{1}} \supset \mathcal{U}_{\gamma_{2}}$. In case $\rho_{\Phi}=\rho_{\Phi}^{(\tau, T)}$, we observe that $\mathcal{R}\left(Q_{\gamma_{1}}\right) \cap \mathcal{N}\left(Q_{\gamma_{2}}\right)=\mathbb{T} \times\{0\}$ holds, because a nonzero element $\xi \in \mathcal{R}\left(Q_{\gamma_{1}}(\tau)\right) \cap \mathcal{N}\left(Q_{\gamma_{2}}(\tau)\right)$ would satisfy

$$
\|\xi\|=\|\Phi(-T, \tau+T) \Phi(T, \tau) \xi\|<e^{-\gamma_{2} T}\|\Phi(T, \tau) \xi\|<e^{-\gamma_{2} T} e^{+\gamma_{1} T}\|\xi\|<\|\xi\| .
$$

This yields $0=\operatorname{dim}\left(\mathcal{R}\left(Q_{\gamma_{1}}\right) \cap \mathcal{N}\left(Q_{\gamma_{2}}\right)\right)=\operatorname{rk} Q_{\gamma_{1}}+\operatorname{dim} \mathcal{N}\left(Q_{\gamma_{2}}\right)-\operatorname{dim}\left(\mathcal{R}\left(Q_{\gamma_{1}}\right)+\mathcal{N}\left(Q_{\gamma_{2}}\right)\right)$, and therefore,

$$
\operatorname{rk} Q_{\gamma_{2}}=\operatorname{rk} Q_{\gamma_{1}}+N-\operatorname{dim}\left(\mathcal{R}\left(Q_{\gamma_{1}}\right)+\mathcal{N}\left(Q_{\gamma_{2}}\right)\right) \geq \operatorname{rk} Q_{\gamma_{1}}
$$

Assume now that $\left[\gamma_{1}, \gamma_{2}\right] \subset \rho_{\Phi}$. Arguing negatively, we suppose that rk $Q_{\gamma_{1}} \neq$ rk $Q_{\gamma_{2}}$. We choose invariant projectors $Q_{\gamma}$ for the nonhyperbolic dichotomies of $(\theta, \varphi)$ with growth rate $\gamma$ for all $\gamma \in\left(\gamma_{1}, \gamma_{2}\right)$ and define

$$
\zeta_{0}:=\sup \left\{\zeta \in\left[\gamma_{1}, \gamma_{2}\right]: \operatorname{rk} Q_{\zeta} \neq \operatorname{rk} Q_{\gamma_{2}}\right\}
$$

Due to Lemma 4.2.7, there exists an $\varepsilon>0$ such that $\operatorname{rk} Q_{\zeta_{0}}=\operatorname{rk} Q_{\zeta}$ for all $\zeta \in U_{\varepsilon}\left(\zeta_{0}\right)$. This is a contradiction to the definition of $\zeta_{0}$. Conversely, let rk $Q_{\gamma_{1}}=\operatorname{rk} Q_{\gamma_{2}}$. We first treat the case $\rho_{\Phi}=\rho_{\Phi}$. Because of rk $Q_{\gamma_{1}}=\operatorname{rk} Q_{\gamma_{2}}$, Proposition 4.1.11 yields that $\mathcal{N}\left(Q_{\gamma_{1}}\right)=\mathcal{N}\left(Q_{\gamma_{2}}\right)$. Due to Lemma 4.2.4, $Q_{\gamma_{2}}$ is an invariant projector of the nonhyperbolic past exponential dichotomy with growth rate $\gamma_{1}$. Thus, we have

$$
\left\|\Phi(t, \tau) Q_{\gamma_{2}}(\tau)\right\| \leq K_{1} e^{\left(\gamma_{1}-\alpha_{1}\right) t} \quad \text { for all } t \geq 0, \tau \leq 0 \text { with } t+\tau \leq 0
$$

for some $K_{1} \geq 1$ and $\alpha_{1}>0 . Q_{\gamma_{2}}$ is also projector of the nonhyperbolic past exponential dichotomy with growth rate $\gamma_{2}$. Hence,

$$
\left\|\Phi(-t, \tau)\left(\mathbb{1}-Q_{\gamma_{2}}(\tau)\right)\right\| \leq K_{2} e^{-\left(\gamma_{2}+\alpha_{2}\right) t} \quad \text { for all } t \geq 0, \tau \leq 0
$$

is fulfilled for some $K_{2} \geq 1$ and $\alpha_{2}>0$. For all $\gamma \in\left[\gamma_{1}, \gamma_{2}\right]$, these two inequalities imply by setting $K:=\max \left\{K_{1}, K_{2}\right\}$ and $\alpha:=\min \left\{\alpha_{1}, \alpha_{2}\right\}$ that

$$
\begin{aligned}
\left\|\Phi(t, \tau) Q_{\gamma_{2}}(\tau)\right\| & \leq K e^{(\gamma-\alpha) t} & & \text { for all } t \geq 0, \tau \leq 0 \text { with } t+\tau \leq 0, \\
\left\|\Phi(-t, \tau)\left(\mathbb{1}-Q_{\gamma_{2}}(\tau)\right)\right\| & \leq K e^{-(\gamma+\alpha) t} & & \text { for all } t \geq 0, \tau \leq 0 .
\end{aligned}
$$

This means $\gamma \in \rho_{\Phi}$, and therefore, $\left[\gamma_{1}, \gamma_{2}\right] \subset \rho_{\Phi}$. The case $\rho_{\Phi}=\rho_{\Phi}, \rho_{\Phi}^{\leftrightarrow}$ is treated analogously. It remains to show the implication for $\rho_{\Phi}=\rho_{\Phi}^{(\tau, T)}$. We have already seen at the beginning of this proof that $\mathcal{R}\left(Q_{\gamma_{1}}\right) \cap \mathcal{N}\left(Q_{\gamma_{2}}\right)=\mathbb{T} \times\{0\}$. Since rk $Q_{\gamma_{1}}=$ rk $Q_{\gamma_{2}}$, this implies the existence of an 
invariant projector $Q$ with $\mathcal{N}(Q)=\mathcal{N}\left(Q_{\gamma_{2}}\right)$ and $\mathcal{R}(Q)=\mathcal{R}\left(Q_{\gamma_{1}}\right)$. Thus, for all $\gamma \in\left[\gamma_{1}, \gamma_{2}\right]$, we have

$$
\begin{aligned}
\|\Phi(T, \tau) \xi\|<e^{\gamma T}\|\xi\| & \text { for all } 0 \neq \xi \in \mathcal{R}(Q(\tau))=\mathcal{R}\left(Q_{\gamma_{1}}(\tau)\right) \\
\|\Phi(-T, \tau+T) \xi\|<e^{-\gamma T}\|\xi\| & \text { for all } 0 \neq \xi \in \mathcal{N}(Q(\tau+T))=\mathcal{N}\left(Q_{\gamma_{2}}(\tau+T)\right)
\end{aligned}
$$

This implies $\left[\gamma_{1}, \gamma_{2}\right] \subset \rho_{\Phi}$ and finishes the proof of this lemma.

For arbitrarily chosen $a \in \mathbb{R}$, we define

$$
\begin{array}{rlrl}
{[-\infty, a]} & :=(-\infty, a] \cup\{-\infty\}, & {[a, \infty]} & :=[a, \infty) \cup\{\infty\}, \\
{[-\infty,-\infty]:} & :=\{-\infty\} & {[\infty, \infty]} & :=\{\infty\}, \\
\text { and } & {[-\infty, \infty]} & =\overline{\mathbb{R}}
\end{array}
$$

We now state the main result of this section.

4.2.9 TheOREM (Spectral TheOREM). For all spectra $\Sigma_{\Phi}:=\Sigma_{\Phi}^{\leftarrow}, \Sigma_{\Phi}, \Sigma_{\Phi}^{\leftrightarrow}, \Sigma_{\Phi}^{(\tau, T)}$ there exists an $n \in\{1, \ldots, N\}$ such that

$$
\Sigma_{\Phi}=\left[a_{1}, b_{1}\right] \cup \cdots \cup\left[a_{n}, b_{n}\right]
$$

with $-\infty \leq a_{1} \leq b_{1}<a_{2} \leq b_{2}<\cdots<a_{n} \leq b_{n} \leq \infty$. In case $\Sigma_{\Phi}=\Sigma_{\Phi}^{(\tau, T)}$, we have $-\infty<a_{1}$ and $b_{n}<\infty$.

Proof. Due to Lemma 4.2.7, the set $\rho_{\Phi} \cap \mathbb{R}$ is open. Therefore, $\Sigma_{\Phi} \cap \mathbb{R}$ is the disjoint union of closed intervals. In case $\Sigma_{\Phi}=\Sigma_{\Phi}^{(\tau, T)}$, the boundedness of $\Sigma_{\Phi}$ is obvious, and if $\Sigma_{\Phi}=$ $\Sigma_{\Phi}^{\leftarrow}, \Sigma_{\Phi}, \Sigma_{\Phi}^{\leftrightarrow}$, respectively, then the relation $\left(-\infty, b_{1}\right] \subset \Sigma_{\Phi}$ implies $\left[-\infty, b_{1}\right] \subset \Sigma_{\Phi}$, because the assumption of the existence of a $\gamma \in \mathbb{R}$ such that $(\theta, \varphi)$ admits a nonhyperbolic dichotomy with growth rate $\gamma$ and projector $Q_{\gamma} \equiv \mathbb{O}$ leads to $(-\infty, \gamma] \subset \rho_{\Phi}$ using Lemma 4.1.9. This is a contradiction. Analogously, it follows from $\left[a_{n}, \infty\right) \subset \Sigma_{\Phi}$ that $\left[a_{n}, \infty\right] \subset \Sigma_{\Phi}$. To show the relation $n \leq N$, we assume that $n \geq N+1$. Thus, there exist

$$
\zeta_{1}<\zeta_{2}<\cdots<\zeta_{N} \in \rho_{\Phi}
$$

such that the $N+1$ intervals

$$
\left(-\infty, \zeta_{1}\right),\left(\zeta_{1}, \zeta_{2}\right), \ldots,\left(\zeta_{N}, \infty\right)
$$

have nonempty intersection with the spectrum $\Sigma_{\Phi}$. It follows from Lemma 4.2.8 that

$$
0 \leq \operatorname{rk} Q_{\zeta_{1}}<\operatorname{rk} Q_{\zeta_{2}}<\cdots<\operatorname{rk} Q_{\zeta_{N}} \leq N
$$

is fulfilled for invariant projectors $Q_{\zeta_{i}}$ of the nonhyperbolic dichotomy with growth rate $\zeta_{i}$, $i \in\{1, \ldots, n\}$. This implies either rk $Q_{\zeta_{1}}=0$ or rk $Q_{\zeta_{N}}=N$. Thus,

$$
\left[-\infty, \zeta_{1}\right] \cap \Sigma_{\Phi}=\emptyset \quad \text { or } \quad\left[\zeta_{N}, \infty\right] \cap \Sigma_{\Phi}=\emptyset,
$$

and this is a contradiction. To show $n \geq 1$, we assume that $\Sigma_{\Phi}=\emptyset$. This implies $\{-\infty, \infty\} \subset \rho_{\Phi}$. Thus, there exist $\zeta_{1}, \zeta_{2} \in \mathbb{R}$ such that $(\theta, \varphi)$ admits a nonhyperbolic dichotomy with growth rate $\zeta_{1}$ and projector $Q_{\zeta_{1}} \equiv \mathbb{O}$ and a nonhyperbolic dichotomy with growth rate $\zeta_{2}$ and projector $Q_{\zeta_{2}} \equiv \mathbb{1}$. 
Applying Lemma 4.2.8, we get $\left(\zeta_{1}, \zeta_{2}\right) \cap \Sigma_{\Phi} \neq \emptyset$. This contradiction yields $n \geq 1$ and finishes the proof of this theorem.

In the following example, spectra of scalar linear differential equations are studied.

4.2.10 EXAMPLES. We consider scalar differential equations of the form

$$
\dot{x}=a(t) x \text {, }
$$

where $a: \mathbb{R} \rightarrow \mathbb{R}$ is a continuous function. We have $\Phi(t, \tau)=\exp \left(\int_{\tau}^{\tau+t} a(s) d s\right)$ for all $t, \tau \in \mathbb{R}$. The Spectral Theorem says that the past, future, all-time and $(\tau, T)$-dichotomy spectra consist of exactly one closed interval. Furthermore, due to Remark 4.1.5 (iv), $\Sigma_{\Phi}^{(\tau, T)}$ simply is the singleton $\{|\Phi(T, \tau)|\}$. The following examples show that the past, future and alltime dichotomy spectra can be more complicated.

(i) $\Sigma_{\Phi}^{\overleftarrow{\Phi}}=\Sigma_{\Phi} \rightarrow=\Sigma_{\Phi}^{\leftrightarrow}=\{\infty\}$ for $a(t):=|t|$ for all $t \in \mathbb{R}$

PROOF. For $\gamma \in \mathbb{R}$, we have

$$
\Phi_{\gamma}(t, \tau)=\exp \left(\int_{\tau}^{\tau+t}(|s|-\gamma) d s\right) \quad \text { for all } t, \tau \in \mathbb{R} .
$$

Since for all $s \in \mathbb{R}$ with $|s| \geq \gamma+1$, the relation $|s|-\gamma \geq 1$ is fulfilled, $\left(\theta, \Phi_{\gamma}\right)$ admits a nonhyperbolic exponential dichotomy on $\mathbb{R}_{-|\gamma|-1}^{\leftarrow}$ and $\mathbb{R}_{|\gamma|+1}^{+}$with growth rate 0 , constants $\alpha=1, K=1$ and invariant projector $\mathbb{O}$. Moreover, Proposition 4.2 .1 (i), (ii) implies that $\Sigma_{\Phi}^{\leftarrow}=\Sigma_{\Phi}=\{\infty\}$. The remaining assertion $\Sigma_{\Phi}^{\overleftrightarrow{ }}=\{\infty\}$ is a consequence of Proposition 4.1.6 (ii).

(ii) $\Sigma_{\Phi}^{\overleftarrow{\Phi}}=\{-\infty\}, \Sigma_{\Phi}=\{\infty\}$ and $\Sigma_{\Phi}^{\overleftrightarrow{ }}=\overline{\mathbb{R}}$ for $a(t):=t$ for all $t \in \mathbb{R}$

PROOF. The assertions concerning the past and future dichotomy spectrum are proved analogously to (i). Concerning the all-time dichotomy spectrum, we assume to the contrary that there exists a $\gamma \in \mathbb{R}$ such that $\Phi_{\gamma}$ admits an all-time exponential dichotomy. Please note that the relation

$$
\Phi_{\gamma}(t, \tau)=\exp \left(\frac{1}{2} t^{2}+\tau t+\gamma t\right) \quad \text { for all } t, \tau \in \mathbb{R}
$$

holds. For the corresponding invariant projector $Q_{\gamma}$, there are only the possibilities $Q_{\gamma} \equiv \mathbb{0}$ or $Q_{\gamma} \equiv \mathbb{1}$. In case $Q_{\gamma} \equiv \mathbb{1}$, the dichotomy estimate

$$
\Phi_{\gamma}(t, 0)=\exp \left(\frac{1}{2} t^{2}+\gamma t\right) \leq K e^{-\alpha t} \text { for all } t \geq 0
$$

yields a contradiction in the limit $t \rightarrow \infty$. Analogously, the case $Q_{\gamma} \equiv \mathbb{O}$ is treated.

(iii) $\Sigma_{\Phi}^{\leftarrow}=[-\infty, \beta], \Sigma_{\Phi}=\{\beta\}$ and $\Sigma_{\Phi}^{\leftrightarrow}=[-\infty, \beta]$ for

$$
a(t):=\left\{\begin{array}{cll}
\beta & : t \geq-1 \\
\beta-n+n\left(t+2^{2 n}+1\right) & : t \in\left[-2^{2 n}-1,-2^{2 n}\right] \text { for some } n \in \mathbb{N}_{0} \\
\beta-n & : t \in\left[-2^{2 n+1},-2^{2 n}-1\right] \text { for some } n \in \mathbb{N}_{0} \\
\beta-n\left(t+2^{2 n+1}+1\right) & : t \in\left[-2^{2 n+1}-1,-2^{2 n+1}\right] \text { for some } n \in \mathbb{N}_{0} \\
\beta & : t \in\left[-2^{2(n+1)},-2^{2 n+1}-1\right] \text { for some } n \in \mathbb{N}_{0}
\end{array} .\right.
$$


PROOF. The statement concerning $\Sigma_{\Phi}$ is clear. To compute $\Sigma_{\Phi}$, assume to the contrary that for some $\gamma \leq \beta$, the linear $\operatorname{NDS}\left(\theta, \Phi_{\gamma}\right)$ admits a past exponential dichotomy with projector $Q_{\gamma}$. In the one-dimensional context, there are only the possibilities $Q_{\gamma} \equiv \mathbb{0}$ or $Q_{\gamma} \equiv \mathbb{1}$. In case $Q_{\gamma} \equiv \mathbb{1}$, we have the dichotomy estimate

$$
\Phi_{\gamma}(t, \tau)=\exp \left(\int_{\tau}^{\tau+t}(a(s)-\gamma) d s\right) \leq K e^{-\alpha t} \quad \text { for all } \tau \leq 0 \text { and } 0 \leq t \leq-\tau
$$

for some $K \geq 1$ and $\alpha>0$. We choose $n \in \mathbb{N}_{0}$ such that $K \exp \left(-\alpha\left(2^{2 n+1}-1\right)\right)<1$. Then,

$$
\Phi_{\gamma}\left(2^{2 n+1}-1,-2^{2(n+1)}\right)=\exp \left(\int_{-2^{2(n+1)}}^{-2^{2 n+1}-1}(\underbrace{\beta-\gamma}_{\geq 0}) d s\right) \geq 1 .
$$

This is a contradiction. In case $Q_{\gamma} \equiv \mathbb{O}$, we have the dichotomy estimate

$$
\Phi_{\gamma}(-t, \tau)=\exp \left(\int_{\tau}^{\tau-t}(a(s)-\gamma) d s\right) \leq K e^{-\alpha t} \quad \text { for all } \tau \leq 0 \text { and } t \geq 0
$$

for some $K \geq 1$ and $\alpha>0$. We choose $n \in \mathbb{N}_{0}$ such that $K \exp \left(-\alpha\left(2^{2 n}-1\right)\right)<1$ and $\beta-n-\gamma \leq 0$. Then,

$$
\Phi_{\gamma}\left(-2^{2 n+1},-2^{2 n}-1\right)=\exp \left(\int_{-2^{2 n}-1}^{-2^{2 n+1}}(\underbrace{\beta-n-\gamma}_{\leq 0}) d s\right) \geq 1 .
$$

This is also a contradiction. It is easy to see that for $\gamma>\beta$, the linear $\operatorname{NDS}\left(\theta, \Phi_{\gamma}\right)$ admits a past exponential dichotomy with projector $Q_{\gamma} \equiv \mathbb{1}$. Hence, we have $\Sigma_{\Phi} \leftarrow=[-\infty, \beta]$. Due to Remark 4.2.6 (iii), $\Sigma_{\Phi}^{\leftrightarrow} \supset \Sigma_{\Phi} \cup \Sigma_{\Phi}=[-\infty, \beta]$ is fulfilled. It is also easily shown that for $\gamma>\beta$, the linear NDS $\left(\theta, \Phi_{\gamma}\right)$ admits an all-time exponential dichotomy with projector $Q_{\gamma} \equiv \mathbb{1}$. Thus, we obtain $\Sigma_{\Phi}^{\leftrightarrow}=[-\infty, \beta]$.

(iv) $\Sigma_{\Phi}^{\leftarrow}=\{\beta\}, \Sigma_{\Phi}=[\beta, \infty]$ and $\Sigma_{\Phi}^{\leftrightarrow}=[\beta, \infty]$ for

$$
a(t):=\left\{\begin{array}{cl}
\beta & : t \leq 1 \\
\beta+n\left(t-2^{2 n}\right) & : t \in\left[2^{2 n}, 2^{2 n}+1\right] \text { for some } n \in \mathbb{N}_{0} \\
\beta+n & : t \in\left[2^{2 n}+1,2^{2 n+1}\right] \text { for some } n \in \mathbb{N}_{0} \\
\beta+n-n\left(t-2^{2 n+1}\right) & : t \in\left[2^{2 n+1}, 2^{2 n+1}+1\right] \text { for some } n \in \mathbb{N}_{0} \\
\beta & : t \in\left[2^{2 n+1}+1,2^{2(n+1)}\right] \text { for some } n \in \mathbb{N}_{0}
\end{array}\right.
$$

PROOF. See proof of (iii).

(v) $\Sigma_{\Phi}^{\overleftarrow{\Phi}}=\{\beta\}, \Sigma_{\Phi}=[\beta, \delta]$ and $\Sigma_{\Phi}^{\leftrightarrow}=[\beta, \delta]$ for

$$
a(t):=\left\{\begin{array}{cl}
\beta & : t \leq 1 \\
\beta+\left(t-2^{2 n}\right)(\delta-\beta) & : t \in\left[2^{2 n}, 2^{2 n}+1\right] \text { for some } n \in \mathbb{N}_{0} \\
\delta & : t \in\left[2^{2 n}+1,2^{2 n+1}\right] \text { for some } n \in \mathbb{N}_{0} \\
\delta+\left(t-2^{2 n+1}\right)(\beta-\delta) & : t \in\left[2^{2 n+1}, 2^{2 n+1}+1\right] \text { for some } n \in \mathbb{N}_{0} \\
\beta & : t \in\left[2^{2 n+1}+1,2^{2(n+1)}\right] \text { for some } n \in \mathbb{N}_{0}
\end{array} .\right.
$$

PROOF. See proof of (iii). 
The following theorem says that each interval of the past (future, all-time, respectively) spectrum corresponds to a linear nonautonomous invariant manifold.

\subsubsection{TheOREM (SPECTRAL MANifolds). Let}

$$
\Sigma_{\Phi}:=\Sigma_{\Phi}^{\leftarrow}, \Sigma_{\Phi}, \Sigma_{\Phi}^{\leftrightarrow}=\left[a_{1}, b_{1}\right] \cup \cdots \cup\left[a_{n}, b_{n}\right]
$$

respectively, define the invariant projectors $Q_{\gamma_{0}}:=\mathbb{O}, Q_{\gamma_{n}}:=\mathbb{1}$, and for $i \in\{1, \ldots, n-1\}$, choose $\gamma_{i} \in\left(b_{i}, a_{i+1}\right)$ and projectors $Q_{\gamma_{i}}$ of the nonhyperbolic dichotomy of $(\theta, \varphi)$ with growth rate $\gamma_{i}$. Then, the sets

$$
\mathcal{W}_{i}:=\mathcal{R}\left(Q_{\gamma_{i}}\right) \cap \mathcal{N}\left(Q_{\gamma_{i-1}}\right) \text { for all } i \in\{1, \ldots, n\}
$$

are linear nonautonomous invariant manifolds, the so-called spectral manifolds, such that

$$
\mathcal{W}_{1} \oplus \cdots \oplus \mathcal{W}_{n}=\mathbb{T} \times \mathbb{R}^{N}
$$

and $\mathcal{W}_{i} \neq \mathbb{T} \times\{0\}$ for $i \in\{1, \ldots, n\}$.

Proof. The sets $\mathcal{W}_{1}, \ldots, \mathcal{W}_{n}$ are obviously linear nonautonomous invariant manifolds. We suppose that there exists an $i \in\{1, \ldots, n\}$ with $\mathcal{W}_{i}=\mathbb{T} \times\{0\}$. In case $i=1$ or $i=n$, Lemma 4.1.9 implies $\left[-\infty, \gamma_{1}\right] \cap \Sigma_{\Phi}=\emptyset$ or $\left[\gamma_{n-1}, \infty\right] \cap \Sigma_{\Phi}=\emptyset$, and this is a contradiction. In case $1<i<n$, due to Lemma 4.2.8, we obtain

$$
\begin{aligned}
\operatorname{dim} \mathcal{W}_{i} & =\operatorname{dim}\left(\mathcal{R}\left(Q_{\gamma_{i}}\right) \cap \mathcal{N}\left(Q_{\gamma_{i-1}}\right)\right) \\
& =\operatorname{rk} Q_{\gamma_{i}}+N-\operatorname{rk} Q_{\gamma_{i-1}}-\operatorname{dim}\left(\mathcal{R}\left(Q_{\gamma_{i}}\right)+\mathcal{N}\left(Q_{\gamma_{i-1}}\right)\right) \geq 1
\end{aligned}
$$

and this is also a contradiction. We now prove $\mathcal{W}_{1} \oplus \cdots \oplus \mathcal{W}_{n}=\mathbb{T} \times \mathbb{R}^{N}$. W.l.o.g., we assume $\Sigma_{\Phi}=\Sigma_{\Phi}, \Sigma_{\Phi}$. For $1 \leq i<j \leq n$, due to Proposition 4.1.11, the relations $\mathcal{W}_{i} \subset \mathcal{R}\left(Q_{\gamma_{i}}\right)$ and $\mathcal{W}_{j} \subset \mathcal{N}\left(Q_{\gamma_{j-1}}\right) \subset \mathcal{N}\left(Q_{\gamma_{i}}\right)$ are fulfilled. This yields

$$
\mathcal{W}_{i} \cap \mathcal{W}_{j} \subset \mathcal{R}\left(Q_{\gamma_{i}}\right) \cap \mathcal{N}\left(Q_{\gamma_{i}}\right)=\mathbb{T} \times\{0\} .
$$

Moreover, Lemma A.2.3 implies that

$$
\begin{aligned}
\mathbb{T} \times \mathbb{R}^{N} & =\mathcal{W}_{1}+\mathcal{N}\left(Q_{\gamma_{1}}\right)=\mathcal{W}_{1}+\mathcal{N}\left(Q_{\gamma_{1}}\right) \cap\left(\mathcal{R}\left(Q_{\gamma_{2}}\right)+\mathcal{N}\left(Q_{\gamma_{2}}\right)\right) \\
& =\mathcal{W}_{1}+\mathcal{N}\left(Q_{\gamma_{1}}\right) \cap \mathcal{R}\left(Q_{\gamma_{2}}\right)+\mathcal{N}\left(Q_{\gamma_{2}}\right)=\mathcal{W}_{1}+\mathcal{W}_{2}+\mathcal{N}\left(Q_{\gamma_{2}}\right)
\end{aligned}
$$

holds. It follows inductively that

$$
\mathbb{T} \times \mathbb{R}^{N}=\mathcal{W}_{1}+\cdots+\mathcal{W}_{n}+\mathcal{N}\left(Q_{\gamma_{n}}\right)=\mathcal{W}_{1}+\cdots+\mathcal{W}_{n}
$$

This finishes the proof of this theorem.

In case of the past and future dichotomy spectrum, the spectral manifolds give rise to a Morse decomposition on the projective space.

4.2.12 Theorem (Spectral Manifolds And Morse Decompositions). Let

$$
\Sigma_{\Phi}=\Sigma_{\Phi}^{\leftarrow}, \Sigma_{\Phi}=\left[a_{1}, b_{1}\right] \cup \cdots \cup\left[a_{n}, b_{n}\right]
$$


respectively, define the invariant projectors $Q_{\gamma_{0}}:=\mathbb{0}, Q_{\gamma_{n}}:=\mathbb{1}$, and for $i \in\{1, \ldots, n-1\}$, choose $\gamma_{i} \in\left(b_{i}, a_{i+1}\right)$ and projectors $Q_{\gamma_{i}}$ of the nonhyperbolic dichotomy of $(\theta, \varphi)$ with growth rate $\gamma_{i}$. Then, the sets

$$
M_{i}:=\mathbb{P}\left(\mathcal{R}\left(Q_{\gamma_{i}}\right) \cap \mathcal{N}\left(Q_{\gamma_{i-1}}\right)\right) \quad \text { for all } i \in\{1, \ldots, n\}
$$

are the Morse sets of a past (future, respectively) Morse decomposition of $(\theta, \mathbb{P} \varphi)$.

PROOF. This is a direct consequence of Theorem 4.1.15.

4.2.13 REMARK. It is possible that the above Morse decomposition defined by the spectral intervals is coarser than the finest Morse decomposition of Theorem 3.5.6 (see also CoLONIUS \& KLIEMANN [48]).

\subsection{LYAPUNOV SPECTRA}

In this section, the so-called Lyapunov spectra are introduced, and their relationship to the past and future dichotomy spectrum is examined. As in the previous section, we restrict to the case that $(\theta, \varphi)$ is generated by a nonautonomous differential or difference equation, i.e., $P=\mathbb{T}=\mathbb{R}, \mathbb{Z}$ and $\theta(t, \tau)=t+\tau$ for all $t, \tau \in \mathbb{T}$.

\subsubsection{DEFINITION (LyAPUNOV EXPONENTS AND LyAPUNOV SPECTRA). For nonzero} $\xi \in \mathbb{R}^{N}$, the numbers

$$
\lambda_{+}^{\leftarrow}(\xi)=\limsup _{t \rightarrow \infty} \frac{1}{t} \ln \|\Phi(-t, 0) \xi\| \quad \text { and } \quad \lambda_{-}^{\leftarrow}(\xi)=\liminf _{t \rightarrow \infty} \frac{1}{t} \ln \|\Phi(-t, 0) \xi\|
$$

are called upper and lower Lyapunov exponent for $t \rightarrow-\infty$. Considering the future, the numbers

$$
\lambda_{+}(\xi)=\limsup _{t \rightarrow \infty} \frac{1}{t} \ln \|\Phi(t, 0) \xi\| \quad \text { and } \quad \lambda_{-}(\xi)=\liminf _{t \rightarrow \infty} \frac{1}{t} \ln \|\Phi(t, 0) \xi\|
$$

are called upper and lower Lyapunov exponent for $t \rightarrow \infty$. The Lyapunov spectrum for $t \rightarrow-\infty$ is defined by

$$
\sigma_{\Phi}^{\leftarrow}:=\bigcup_{0 \neq \xi \in \mathbb{R}^{N}}\left[\lambda_{-}^{\leftarrow}(\xi), \lambda_{+}^{\leftarrow}(\xi)\right]
$$

and the Lyapunov spectrum for $t \rightarrow \infty$ is defined by

$$
\sigma_{\Phi}:=\bigcup_{0 \neq \xi \in \mathbb{R}^{N}}\left[\lambda_{-}(\xi), \lambda_{+}(\xi)\right]
$$

It is well-known that there exist $n^{-}, n^{+} \in\{1, \ldots, N\}$ and $\xi_{1}^{-}, \ldots, \xi_{n^{-}}^{-}, \xi_{1}^{+}, \ldots, \xi_{n^{+}}^{+} \in \mathbb{R}^{N}$ such that

$$
\sigma_{\Phi}^{\leftarrow}=\left[\lambda_{-}^{\leftarrow}\left(\xi_{1}\right), \lambda_{+}^{\leftarrow}\left(\xi_{1}\right)\right] \cup \cdots \cup\left[\lambda_{-}^{\leftarrow}\left(\xi_{n^{-}}\right), \lambda_{+}^{\leftarrow}\left(\xi_{n^{-}}\right)\right]
$$


and

$$
\sigma_{\Phi}=\left[\lambda_{-}^{\rightarrow}\left(\xi_{1}\right), \lambda_{+}\left(\xi_{1}\right)\right] \cup \cdots \cup\left[\lambda_{-}^{\rightarrow}\left(\xi_{n^{+}}\right), \lambda_{+}\left(\xi_{n^{+}}\right)\right]
$$

(see, e.g., BARREIRA \& PESIN [24] and Dieci \& VAN VLeCK [61, 62]).

In the following, the relationship between the dichotomy spectra and the Lyapunov spectra is discussed.

\subsubsection{Theorem (Relationship to the Past And Future Dichotomy Spectrum).} The relations $-\sigma_{\Phi}^{\leftarrow} \subset \Sigma_{\Phi}^{\leftarrow}$ and $\sigma_{\Phi} \subset \Sigma_{\Phi}$ hold

Proof. Let $\lambda \in-\sigma_{\Phi}^{\leftarrow}$. Thus, there exists a $\xi \in \mathbb{R}^{N}$ with $-\lambda \in\left[\lambda_{-}(\xi), \lambda_{+}^{\leftarrow}(\xi)\right]$. Initially we suppose that $\lambda \in \mathbb{R}$. Arguing negatively, we assume, $(\theta, \varphi)$ admits a nonhyperbolic past exponential dichotomy with growth rate $\gamma:=\lambda$, constants $K \geq 1, \alpha>0$ and invariant projector $Q_{\gamma}$, i.e.,

$$
\begin{aligned}
\left\|\Phi(t, \tau) Q_{\gamma}(\tau)\right\| & \leq K e^{(\gamma-\alpha) t} & & \text { for all } \tau \leq 0 \text { and } t \geq 0 \text { with } \tau+t \leq 0, \\
\left\|\Phi(-t, \tau)\left(\mathbb{1}-Q_{\gamma}(\tau)\right)\right\| & \leq K e^{-(\gamma+\alpha) t} & & \text { for all } \tau \leq 0 \text { and } t \geq 0 .
\end{aligned}
$$

We write $\xi=\xi_{1}+\xi_{2}$ with $\xi_{1} \in \mathcal{R}\left(Q_{\gamma}(0)\right)$ and $\xi_{2} \in \mathcal{N}\left(Q_{\gamma}(0)\right)$. In case $\xi_{1}=0$, we have

$$
\begin{aligned}
\lambda_{+}^{\leftarrow}(\xi) & =\limsup _{t \rightarrow \infty} \frac{1}{t} \ln \|\Phi(-t, 0) \xi\| \leq \limsup _{t \rightarrow \infty} \frac{1}{t} \ln \left(K e^{-(\gamma+\alpha) t}\right) \\
& =-\gamma-\alpha=-\lambda-\alpha \leq \lambda_{+}^{\leftarrow}(\xi)-\alpha .
\end{aligned}
$$

This is a contradiction. Otherwise $\left(\xi_{1} \neq 0\right)$, we observe that for all $t \geq 0$,

$$
\begin{aligned}
\left\|\xi_{1}\right\| & =\left\|Q_{\gamma}(0) \xi\right\|=\left\|\Phi(t,-t) \Phi(-t, 0) Q_{\gamma}(0) \xi\right\|=\left\|\Phi(t,-t) Q_{\gamma}(-t) \Phi(-t, 0) \xi\right\| \\
& \leq K e^{(\gamma-\alpha) t}\|\Phi(-t, 0) \xi\|
\end{aligned}
$$

is fulfilled. Thus, $\|\Phi(-t, 0) \xi\| \geq K^{-1} e^{-(\gamma-\alpha) t}\left\|\xi_{1}\right\|$ for all $t \geq 0$, and therefore,

$$
\begin{aligned}
\lambda_{-}^{\leftarrow}(\xi) & =\liminf _{t \rightarrow \infty} \frac{1}{t} \ln \|\Phi(-t, 0) \xi\| \geq \liminf _{t \rightarrow \infty} \frac{1}{t} \ln \left(K^{-1} e^{-(\gamma-\alpha) t}\left\|\xi_{1}\right\|\right) \\
& =\alpha-\gamma=\alpha-\lambda \geq \alpha+\lambda_{-}^{\leftarrow}(\xi) .
\end{aligned}
$$

This is also a contradiction, and hence, $\lambda \in \Sigma_{\Phi}^{\leftarrow}$. We now treat the case $\lambda \notin \mathbb{R}$, w.l.o.g., $\lambda=\infty$ Assume that $-\infty \notin \Sigma_{\Phi}$. Thus, there exist $\gamma \in \mathbb{R}, K \geq 1$ and $\alpha>0$ with

$$
\|\Phi(-t, 0)\| \leq K e^{-(\gamma+\alpha) t} \quad \text { for all } t \geq 0,
$$

and this relation implies the contradiction

$$
\limsup _{t \rightarrow \infty} \frac{1}{t} \ln (\|\Phi(-t, 0)\|) \leq-\gamma-\alpha<\infty
$$

Hence, $\lambda \in \Sigma_{\Phi}^{\leftarrow}$. The remaining assertion $\sigma_{\Phi} \subset \Sigma_{\Phi}$ can be proved analogously

The following example shows that the Lyapunov spectra do not coincide with the dichotomy spectra. 
4.3.3 EXAMPLE. We consider the scalar linear nonautonomous differential equation

$$
\dot{x}=(t \sin (t)-\cos (t)) x,
$$

which generates a linear nonautonomous dynamical system with $P=\mathbb{R}$. We have

$$
\Phi(t, \tau)=\exp (-(t+\tau) \cos (t+\tau)+\tau \cos (\tau)) \text { for all } t, \tau \in \mathbb{R} .
$$

An easy calculation yields $\sigma_{\Phi}^{\leftarrow}=\sigma_{\Phi} \rightarrow[-1,1]$. Choosing

$$
t_{1}:=\frac{\pi}{2}, t_{2}:=-\frac{\pi}{2}, \tau_{1}:=2 k \pi-\frac{\pi}{2}, \tau_{2}:=2 k \pi+\frac{\pi}{2} \quad \text { for all } k \in \mathbb{Z},
$$

we obtain

$$
\Phi\left(t_{1}, \tau_{1}\right)=\Phi\left(t_{2}, \tau_{2}\right)=\exp (2 k \pi) .
$$

Hence, for any $\gamma \in \mathbb{R}$, this system does not admit a nonhyperbolic past exponential dichotomy with growth rate $\gamma$. This implies $\Sigma_{\Phi}^{\overleftarrow{ }}=\overline{\mathbb{R}}$. Analogously, one can show that $\Sigma_{\Phi}=\overline{\mathbb{R}}$ is fulfilled

\subsection{Spectra of Autonomous Linear Systems}

It is well-known that an autonomous linear differential equation

$$
\dot{x}=A x
$$

with a matrix $A \in \mathbb{R}^{N \times N}$ admits an exponential dichotomy on $\square=\mathbb{R}_{0}^{-}, \mathbb{R}_{0}^{+}, \mathbb{R}$, respectively, if and only if the real part of every eigenvalue of $A$ is unequal to zero (see, e.g., KALKBRENNER [87, Satz 1.1.3.2, p. 24] and SACKER \& SELL [150, p. 430(1)]). Therefore, the corresponding past, future and all-time dichotomy spectra satisfy

$$
\Sigma_{A}^{\overleftarrow{A}}=\Sigma_{\vec{A}}=\Sigma_{\vec{A}}^{\leftrightarrow}=\{\operatorname{Re} \lambda: \lambda \text { is an eigenvalue of } A\}
$$

A relation of this kind does not hold for the $(\tau, T)$-dichotomy spectrum. Nevertheless, by letting $T$ tend to $\infty$, we obtain the following statement.

4.4.1 Theorem (Spectra of Autonomous Linear Systems). Consider the linear system (4.4). Then, the limit relation

$$
\lim _{T \rightarrow \infty} \Sigma_{A}^{(0, T)}=\{\operatorname{Re} \lambda: \lambda \text { is an eigenvalue of } A\}
$$

holds with respect to the Hausdorff distance.

PROOF. There exist $n \in\{1, \ldots, N\}$ and reals $\lambda_{1}<\lambda_{2}<\cdots<\lambda_{n}$ with

$$
\{\operatorname{Re} \lambda: \lambda \text { is an eigenvalue of } A\}=\left\{\lambda_{1}, \ldots, \lambda_{n}\right\} .
$$

It is sufficient to show that for all $\varepsilon>0$, there exists a $\tau>0$ with

$$
\left\{\lambda_{1}, \ldots, \lambda_{n}\right\} \subset U_{\varepsilon}\left(\Sigma_{A}^{(0, T)}\right) \quad \text { and } \quad \Sigma_{A}^{(0, T)} \subset \bigcup_{i=1}^{n} U_{\varepsilon}\left(\lambda_{i}\right) \text { for all } T \geq \tau
$$


Let $\varepsilon>0$. It is an elementary result in the theory of linear differential equations (see, e.g., COPPEL $\left[53\right.$, p. 56]) that there exist nontrivial linear subspaces $U_{1}, \ldots, U_{n} \subset \mathbb{R}^{N}$ with $U_{1} \oplus \cdots \oplus U_{n}=\mathbb{R}^{N}$ and a real constant $K \geq 1$ such that for all $i \in\{1, \ldots, n\}$,

$$
\begin{aligned}
\left\|e^{A t} \xi\right\| \leq K e^{\left(\lambda_{i}+\frac{\varepsilon}{4}\right) t}\|\xi\| & \text { for all } \xi \in U_{1} \oplus \cdots \oplus U_{i} \text { and } t \geq 0 \\
\left\|e^{A t} \xi\right\| \geq \frac{1}{K} e^{\left(\lambda_{i}-\frac{\varepsilon}{4}\right) t}\|\xi\| & \text { for all } \xi \in U_{i} \oplus \cdots \oplus U_{n} \text { and } t \geq 0
\end{aligned}
$$

is fulfilled. We choose $\tau>0$ and $T \geq \tau$ with $K e^{-\frac{\varepsilon}{4} \tau}<1$.

To prove the first condition of (4.5), we choose an $i \in\{1, \ldots, n\}$ and assume to the contrary that $U_{\varepsilon}\left(\lambda_{i}\right) \cap \Sigma_{A}^{(0, T)}=\emptyset$. Thus, there exists an invariant projector $Q_{\left(\lambda_{i}-\frac{\varepsilon}{2}\right)}$ with

$$
\left\|e^{A T} \xi\right\|<e^{\left(\lambda_{i}-\frac{\varepsilon}{2}\right) T}\|\xi\| \quad \text { for all } 0 \neq \xi \in \mathcal{R}\left(Q_{\left(\lambda_{i}-\frac{\varepsilon}{2}\right)}(0)\right)
$$

and an invariant projector $Q_{\left(\lambda_{i}+\frac{\varepsilon}{2}\right)}$ with

$$
\left\|e^{-A T} \xi\right\|<e^{-\left(\lambda_{i}+\frac{\varepsilon}{2}\right) T}\|\xi\| \quad \text { for all } 0 \neq \xi \in \mathcal{N}\left(Q_{\left(\lambda_{i}+\frac{\varepsilon}{2}\right)}(T)\right)
$$

Because of Lemma 4.2.8, we have $\operatorname{rk} Q_{\left(\lambda_{i}-\frac{\varepsilon}{2}\right)}=\operatorname{rk} Q_{\left(\lambda_{i}+\frac{\varepsilon}{2}\right)}=: r$. If $r \geq \operatorname{dim} U_{1}+\cdots+\operatorname{dim} U_{i}$, then $\operatorname{dim}\left(\mathcal{R}\left(Q_{\left(\lambda_{i}-\frac{\varepsilon}{2}\right)}(0)\right) \cap\left(U_{i} \oplus \cdots \oplus U_{n}\right)\right) \geq 1$. In this case, there exists a nonzero element $\xi \in \mathcal{R}\left(Q_{\left(\lambda_{i}-\frac{\varepsilon}{2}\right)}(0)\right) \cap\left(U_{i} \oplus \cdots \oplus U_{n}\right)$, and this leads to the contradiction

$$
\begin{aligned}
\|\xi\| & =e^{-\left(\lambda_{i}-\frac{\varepsilon}{2}\right) T} e^{\left(\lambda_{i}-\frac{\varepsilon}{2}\right) T}\|\xi\| \stackrel{(4.8)}{>} e^{-\left(\lambda_{i}-\frac{\varepsilon}{2}\right) T}\left\|e^{A T} \xi\right\| \stackrel{(4.7)}{\geq} e^{-\left(\lambda_{i}-\frac{\varepsilon}{2}\right) T} \frac{1}{K} e^{\left(\lambda_{i}-\frac{\varepsilon}{4}\right) T}\|\xi\| \\
& =\left(K e^{-\frac{\varepsilon}{4} T}\right)^{-1}\|\xi\|>\|\xi\| .
\end{aligned}
$$

If $r<\operatorname{dim} U_{1}+\cdots+\operatorname{dim} U_{i}$, then we have $\operatorname{dim}\left(\mathcal{N}\left(Q_{\left(\lambda_{i}+\frac{\varepsilon}{2}\right)}(0)\right) \cap\left(U_{1} \oplus \cdots \oplus U_{i}\right)\right) \geq 1$. Thus, there exists a nontrivial element $\xi \in \mathcal{N}\left(Q_{\left(\lambda_{i}+\frac{\varepsilon}{2}\right)}(0)\right) \cap\left(U_{1} \oplus \cdots \oplus U_{i}\right)$, and this also yields the contradiction

$$
\|\xi\|=\left\|e^{-A T} e^{A T} \xi\right\| \stackrel{(4.9)}{<} e^{-\left(\lambda_{i}+\frac{\varepsilon}{2}\right) T}\left\|e^{A T} \xi\right\| \stackrel{(4.6)}{\leq} e^{-\left(\lambda_{i}+\frac{\varepsilon}{2}\right) T} K e^{\left(\lambda_{i}+\frac{\varepsilon}{4}\right) T}\|\xi\|=K e^{-\frac{\varepsilon}{4} T}\|\xi\|<\|\xi\| .
$$

To prove the second condition of (4.5), let $\lambda \notin \cup_{i=1}^{n} U_{\varepsilon}\left(\lambda_{i}\right)$. We set $\lambda_{0}:=-\infty$ and $\lambda_{n+1}:=\infty$. There exists a $i \in\{0, \ldots, n\}$ such that

$$
\lambda \geq \lambda_{i}+\varepsilon \quad \text { and } \quad \lambda \leq \lambda_{i+1}-\varepsilon
$$

Now, we define the invariant projector $Q$ by

$$
\mathcal{R}(Q(0))=U_{1} \oplus \cdots \oplus U_{i} \quad \text { and } \quad \mathcal{N}(Q(0))=U_{i+1} \oplus \cdots \oplus U_{n}
$$

Thus, for all nonzero $\xi \in \mathcal{R}(Q(0))$, we have

$$
\left\|e^{A T} \xi\right\| \stackrel{(4.6)}{\leq} K e^{\left(\lambda_{i}+\frac{\varepsilon}{4}\right) T}\|\xi\| \leq K e^{\left(\lambda-\frac{3 \varepsilon}{4}\right) T}\|\xi\|<e^{\lambda T}\|\xi\|,
$$

and for all nonzero $\xi \in \mathcal{N}(Q(T))$,

$$
\left\|e^{-A T} \xi\right\| \stackrel{(4.7)}{\leq} K e^{-\left(\lambda_{i+1}-\frac{\varepsilon}{4}\right) T}\|\xi\| \leq K e^{-\left(\lambda+\frac{3 \varepsilon}{4}\right) T}\|\xi\|<e^{-\lambda T}\|\xi\|
$$

is fulfilled. Hence, $\lambda \notin \Sigma_{A}^{(0, T)}$, and this finishes the proof of this theorem. 
4.4.2 Remark. Using Floquet Theory (see, e.g., Coddington \& LeVinson [47, pp. 78-80] or CHICONE [44, Section 2.4, pp. 162-197]), one can extend the above theorem to periodic linear differential systems of the form

$$
\dot{x}=A(t) x \text {, }
$$

where $A: \mathbb{R} \rightarrow \mathbb{R}^{N \times N}$ fulfills $A(t)=A(t+\omega)$ for all $t \in \mathbb{R}$ with some $\omega>0$. We denote the transition operator of (4.10) by $\Lambda$. For the past (future, all-time, respectively) dichotomy spectrum, we obtain

$$
\Sigma_{A}^{\overleftarrow{A}}=\Sigma_{A} \vec{A}=\Sigma_{A}^{\leftrightarrow}=\{\ln |\lambda|: \lambda \text { is an eigenvalue of } \Lambda(\omega, 0)\}
$$

The matrix $\Lambda(\omega, 0)$ is called monodromy matrix of (4.10). The $(0, T)$-dichotomy spectrum fulfills limit relation

$$
\lim _{T \rightarrow \infty} \Sigma_{A}^{(0, T)}=\{\ln |\lambda|: \lambda \text { is an eigenvalue of } \Lambda(\omega, 0)\}
$$

in the sense of Hausdorff distance.

4.4.3 EXAmple. For fixed $T>0$, we want to compute the $(0, T)$-dichotomy spectrum $\Sigma_{A}^{(0, T)}$ of system (4.4), where

$$
A:=\left(\begin{array}{ll}
1 & 1 \\
0 & 1
\end{array}\right)
$$

Specifically, in this example, we use the norm $\|\cdot\|_{1}: \mathbb{R}^{2} \rightarrow \mathbb{R}_{0}^{+},\left\|\left(x_{1}, x_{2}\right)\right\|_{1}:=\left|x_{1}\right|+\left|x_{2}\right|$. Please note that, for $\gamma \in \mathbb{R}$, the relation

$$
e^{(A-\gamma \mathbb{1}) T}=\left(\begin{array}{cc}
e^{(1-\gamma) T} & T e^{(1-\gamma) T} \\
0 & e^{(1-\gamma) T}
\end{array}\right)
$$

is fulfilled (see, e.g., AULBACH [14]). Hence, for all $\xi=\left(\xi_{1}, \xi_{2}\right) \in \mathbb{R}^{2}$ with $\|\xi\|_{1}=1$, we have

$$
\underbrace{\left\|e^{(A-\gamma \mathbb{1}) T}\left(\begin{array}{l}
1 \\
0
\end{array}\right)\right\|_{1}}_{=e^{(1-\gamma) T}} \leq\left\|e^{(A-\gamma \mathbb{1}) T}\left(\begin{array}{l}
\xi_{1} \\
\xi_{2}
\end{array}\right)\right\|_{1} \leq \underbrace{\left\|e^{(A-\gamma \mathbb{1}) T}\left(\begin{array}{l}
0 \\
1
\end{array}\right)\right\|_{1}}_{=T e^{(1-\gamma) T}+e^{(1-\gamma) T}} .
$$

The term $T e^{(1-\gamma) T}+e^{(1-\gamma) T}$ is strictly monotone decreasing in $\gamma \in \mathbb{R}$, and therefore, there exists a uniquely determined $\gamma_{*}=\gamma_{*}(T)>1$ with $T e^{\left(1-\gamma_{*}\right) T}+e^{\left(1-\gamma_{*}\right) T}=1$.

Using these observations, it is easy to see that $\Sigma_{A}^{(0, T)}=\left\{1, \gamma_{*}\right\}$, since

- for $\gamma<1$, the linear system (4.4) admits a nonhyperbolic $(0, T)$-dichotomy with growth rate $\gamma$ and invariant projector $Q_{\gamma} \equiv \mathbb{0}$,

- for $\gamma \in\left(1, \gamma_{*}\right)$, the linear system (4.4) admits a nonhyperbolic $(0, T)$-dichotomy with growth rate $\gamma$ and invariant projector $Q_{\gamma}$, determined by $\mathcal{R}\left(Q_{\gamma}(0)\right)=\{\beta(1,0): \beta \in \mathbb{R}\}$ and $\mathcal{N}\left(Q_{\gamma}(0)\right)=\{\beta(0,1): \beta \in \mathbb{R}\}$.

- for $\gamma>\gamma_{*}$, the linear system (4.4) admits a nonhyperbolic $(0, T)$-dichotomy with growth rate $\gamma$ and invariant projector $Q_{\gamma} \equiv \mathbb{1}$,

- for $\gamma \in\left\{1, \gamma_{*}\right\}$, the linear system (4.4) admits no nonhyperbolic $(0, T)$-dichotomy with growth rate $\gamma$.

Please note that Theorem 4.4.1 implies that $\lim _{T \rightarrow \infty} \gamma_{*}(T)=1$. 


\subsection{Roughness}

We consider the nonautonomous linear differential equation

$$
\dot{x}=A(t) x
$$

with a continuous function $A: \mathbb{R} \rightarrow \mathbb{R}^{N \times N}$ and a perturbed system

$$
\dot{x}=(A(t)+B(t)) x
$$

with a continuous function $B: \mathbb{R} \rightarrow \mathbb{R}^{N \times N}$. The transition operators of (4.11) and (4.12) are denoted by $\Lambda$ and $\Lambda^{*}$, respectively.

4.5.1 THEOREM (Roughness TheOrem For NONHyperbolic EXPONENTIAL DiCHOTOMIES ON $\square$ ). Let $\square$ be an unbounded and closed interval, and suppose, (4.11) admits a nonhyperbolic exponential dichotomy on $\llbracket$ with growth rate $\gamma$, constants $\alpha, K$ and invariant projector $Q$. If the relation

$$
\delta:=\sup _{t \in \llbracket}\|B(t)\|<\frac{\alpha}{4 K^{2}}
$$

is fulfilled, then also the perturbed system (4.12) admits a nonhyperbolic exponential dichotomy on $\llbracket$ with growth rate $\gamma$, constants $(\alpha-2 K \delta), \frac{5 K^{2}}{2}$ and an invariant projector $\hat{Q}$, more precisely, we have

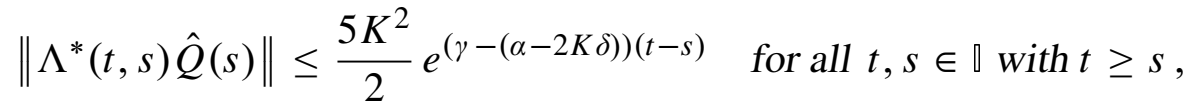

$$
\begin{aligned}
& \left\|\Lambda^{*}(t, s)(\mathbb{1}-\hat{Q}(s))\right\| \leq \frac{5 K^{2}}{2} e^{(\gamma+\alpha-2 K \delta)(t-s)} \quad \text { for all } t, s \in \mathbb{\square} \text { with } t \leq s .
\end{aligned}
$$

In case $\mathbb{\square}=\mathbb{R}_{\kappa}^{-}$, the invariant projector $\hat{Q}$ has the same image as $Q$, and if $\square=\mathbb{R}_{\kappa}^{+}$holds, then $\hat{Q}$ has the same null space as $Q$. Finally, if $\llbracket=\mathbb{R}$ is fulfilled, we get rk $\hat{Q}=\operatorname{rk} Q$.

PROOF. See CopPel [54, Proposition 1, p. 34] or CopPel [53].

4.5.2 REMARK. The perturbations considered in this theorem are perturbations with respect to the uniform topology, generated by the norm

$$
\|A\|_{\infty}:=\sup _{t \in \rrbracket}\|A(t)\| \quad \text { for all } A \in C\left(\mathbb{\square}, \mathbb{R}^{N \times N}\right),
$$

where $C\left(\square, \mathbb{R}^{N \times N}\right):=\left\{X: \llbracket \rightarrow \mathbb{R}^{N \times N}: X\right.$ is continuous $\}$. It is possible to weaken this condition on the perturbation (see, e.g., PÖTZSCHE [138] or PLISS \& SELL [132]). For instance, considering the topology of uniform convergence on compact sets, i.e., $\lim _{n \rightarrow \infty} A_{n}=A_{0}$ if and only if

$$
\lim _{n \rightarrow \infty} \sup _{t \in \rrbracket}\left\|A_{n}(t)-A_{0}(t)\right\|=0 \quad \text { for all compact sets } \rrbracket \subset \square,
$$

one can derive a similar but more stronger perturbation result as Theorem 4.5.1 (see also SACKER \& SELL [153, Section 5, Remark on p. 346]).

4.5.3 Theorem (Roughness Theorem For Nonhyperbolic $(\tau, T)$-DichotoMIES). Suppose, (4.11) admits a nonhyperbolic $(\tau, T)$-dichotomy with growth rate $\gamma$ and projector $Q$. Then, there exists an $\varepsilon>0$ with the following property: If 


$$
\sup _{t \in[\tau, \tau+T]}\|B(t)\|<\varepsilon,
$$

then also the perturbed system (4.12) admits a nonhyperbolic $(\tau, T)$-dichotomy with growth rate $\gamma$ and projector $Q$.

ProOF. This statement follows directly from the continuity of the general solution (cf. Proposition A.1.3). 



\section{CHAPTER 5}

\section{NONLINEAR SYSTEMS}

In the study of nonlinear systems, invariant manifolds play a central role, since it helps to understand the often complicated dynamical behavior near an equilibrium, a periodic solution or-in the nonautonomous context - an arbitrary solution. The construction of stable and unstable invariant manifolds goes back to POINCARÉ [135] and HADAMARD [72]. In the sequel, the theory was extended from hyperbolic to nonhyperbolic systems, from finite to infinite dimension and from time-independent to time-dependent equations.

To mention only few references of the comprehensive amount of literature for autonomous differential equations, we refer to CARR [38], CHOW \& Li \& WANG [46], HiRsch \& PugH \& Shub [79], Kelley [90, 91], Kircharaber \& Palmer [92], Pliss [131], Shub [169], VAnDERBAUWHEDE [179] and WigGINS [182]. In the nonautonomous context, see AULBACH [13], Aulbach \& Wanner [21], Sell [167], Wanner [180] and Yi [183].

In the first section of this chapter, invariant manifolds are constructed which apply to different time domains. It suffices to extend the results of AULBACH \& WANNER [21] and SIEGMUND [170] slightly. Also, the relationship to the notions of attractivity and repulsivity is discussed. In Section 5.2, these results are applied in the context of nonautonomous bifurcation theory. It is shown that under special assumptions, zero is contained in the dichotomy spectrum of the linearization of a bifurcating solution. In Section 5.3, properties of attraction and repulsion for nonlinear systems are derived from the study of the linearization, and finally, Section 5.4 is devoted to the relationship between the bifurcation theory of adiabatic systems and the concept of finitetime bifurcation.

\subsection{Nonautonomous InVARIANT MaNifoldS}

Let $\square$ be an unbounded interval of the form $\mathbb{R}, \mathbb{R}_{\mathcal{\kappa}}^{-}$or $\mathbb{R}_{\mathcal{\kappa}}^{+}$, respectively. In this section, we consider nonlinear differential equations of the form

$$
\dot{x}=A(t) x+F(t, x)
$$

with a continuous function $A: \llbracket \rightarrow \mathbb{R}^{N \times N}$ and a $C^{1}$-function $F: \llbracket \times U \rightarrow \mathbb{R}^{N}$, where $U$ is an open neighborhood of 0 and $F(t, 0)=0$ for all $t \in \mathbb{\text { l }}$. The general solution of (5.1) will be denoted by $\lambda$. In addition to $(5.1)$, we consider the corresponding linear differential equation

$$
\dot{x}=A(t) x
$$




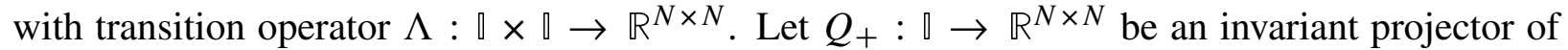

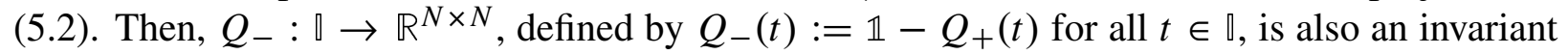
projector.

Please note that in the following, the symbol $Q_{ \pm}$simultaneously stands for $Q_{+}$and $Q_{-}$, respectively. We proceed similarly with our further notation in this section.

Next, we introduce a nonautonomous counterpart of an invariant manifold for (5.1).

5.1.1 Definition (Nonautonomous InVariant Manifolds). Assume that for an interval $\square \subset \mathbb{R}$ and a neighborhood $V$ of $0, C^{1}$-functions $s^{ \pm}: \llbracket \times V \rightarrow \mathbb{R}^{N}$ satisfy

(i) $s^{ \pm}(t, 0)=0$ for all $t \in \mathbb{\square}$,

(ii) $\lim _{x \rightarrow 0} \frac{s^{ \pm}(t, x)}{\|x\|}=0$ uniformly in $t \in \mathbb{\square}$,

(iii) $s^{ \pm}(t, x)=s^{ \pm}\left(t, Q_{ \pm}(t) x\right) \in \mathcal{R}\left(Q_{\mp}(t)\right)$ for all $t \in \mathbb{\square}$ and $x \in V$.

Then, the graphs

$$
\mathcal{S}^{ \pm}:=\left\{\left(\tau, \xi+s^{ \pm}(\tau, \xi)\right) \in \mathbb{\square} \times \mathbb{R}^{N}: \xi \in \mathcal{R}\left(Q_{ \pm}(\tau)\right) \cap V\right\}
$$

are called (local) nonautonomous invariant manifolds of (5.1) if

$$
\begin{array}{ll}
(t, \lambda(t, \tau, \xi)) \in \mathcal{S}^{ \pm} \quad & \text { for all }(\tau, \xi) \in \mathcal{S}^{ \pm} \text {and } t \in \mathbb{\square} \text { such that } \\
& \lambda(\tau+c(t-\tau), \tau, \xi) \in V \text { for all } c \in[0,1] .
\end{array}
$$

We call $\mathcal{S}^{ \pm}$global nonautonomous invariant manifolds if $V=\mathbb{R}^{N}$.

Now, existence results for nonautonomous manifolds of (5.1) are proved and applications are discussed with respect to the notions of attractivity and repulsivity introduced in Chapter 2 . Before doing so, some hypotheses on the linear part and the nonlinearity are needed.

We assume, the following hypotheses hold:

- Hypothesis on Linear Part. The linear system (5.2) admits a nonhyperbolic alltime (past, future, respectively) exponential dichotomy, more precisely, there exists an invariant projector $Q_{+}: \rrbracket \rightarrow \mathbb{R}^{N \times N}$ such that the inequalities

$$
\begin{array}{ll}
\left\|\Lambda(t, s) Q_{+}(s)\right\| \leq K e^{\alpha(t-s)} & \text { for all } t \geq s \\
\left\|\Lambda(t, s) Q_{-}(s)\right\| \leq K e^{\beta(t-s)} & \text { for all } t \leq s
\end{array}
$$

hold with real constants $K \geq 1$ and $\alpha<\beta$.

- Hypothesis on Nonlinearity. There exists a monotone increasing function $\Gamma:(0,1) \rightarrow \overline{\mathbb{R}}^{+}$with $\lim _{s \triangleleft 0} \Gamma(s)=0$ and

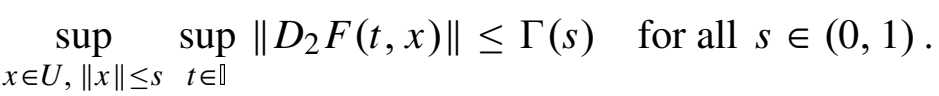

5.1.2 REMARK. The Hypothesis on nonlinearity also reads as $\lim _{x \rightarrow 0} \sup _{t \in \emptyset}\left\|D_{2} F(t, x)\right\|=0$. In the above description, the function $\Gamma$ is needed to explain the dependence of some constants in the next theorems concerning the rate of this limit process. 
First, the case of all-time invariant manifolds of (5.1) is treated.

5.1.3 TheOREM (All-Time InVARIANt MANifolds). In case $\mathbb{q}=\mathbb{R}$, there exist $\rho>0$ and $C^{1}$-functions $s^{ \pm}: \mathbb{R} \times U_{\rho}(0) \rightarrow \mathbb{R}^{N}$ such that the graphs

$$
\mathcal{S}^{ \pm}:=\left\{\left(\tau, \xi+s^{ \pm}(\tau, \xi)\right) \in \mathbb{R} \times \mathbb{R}^{N}: \xi \in \mathcal{R}\left(Q_{ \pm}(\tau)\right) \cap U_{\rho}(0)\right\}
$$

are local nonautonomous invariant manifolds. Furthermore, the following statements are fulfilled:

(i) Case $\alpha<0$ and rk $Q_{+} \geq 1$ (Trivial solution is not all-time repulsive). For all $\varepsilon>0$, there exists an $r>0$ such that for all $(\tau, \xi) \in \mathcal{S}^{+}$with $\|\xi\|<r$, we have

$$
\lambda(t, \tau, \xi) \in U_{\rho}(0) \text { for all } t \geq \tau \quad \text { and } \quad \lambda(\cdot, \tau, \xi) \text { is }(\alpha+\varepsilon)^{+} \text {-quasibounded. }
$$

(ii) Case $\beta>0$ and rk $Q_{-} \geq 1$ (Trivial solution is not all-time attractive). For all $\varepsilon>0$, there exists an $r>0$ such that for all $(\tau, \xi) \in \mathcal{S}^{-}$with $\|\xi\|<r$, we have

$$
\lambda(t, \tau, \xi) \in U_{\rho}(0) \text { for all } t \leq \tau \text { and } \lambda(\cdot, \tau, \xi) \text { is }(\beta-\varepsilon)^{-} \text {-quasibounded }
$$

(iii) Case $\alpha<0$ and $Q_{+} \equiv \mathbb{1}$ (Trivial solution is all-time attractive). There exists an $r=r(\alpha, K, \Gamma)>0$ with

$$
\lim _{t \rightarrow \infty} \sup _{\tau \in \mathbb{R}} d\left(\lambda\left(\tau+t, \tau, U_{r}(0)\right) \mid\{0\}\right)=0 .
$$

(iv) Case $\beta>0$ and $Q_{-} \equiv \mathbb{1}$ (Trivial solution is all-time repulsive). There exists an $r=r(\beta, K, \Gamma)>0$ with

$$
\lim _{t \rightarrow \infty} \sup _{\tau \in \mathbb{R}} d\left(\lambda\left(\tau-t, \tau, U_{r}(0)\right) \mid\{0\}\right)=0 .
$$

PROOF. The proof is divided into two steps.

STEP 1. Existence of $\mathcal{S}^{ \pm}$.

In case $Q_{+} \equiv \mathbb{0}$ or $Q_{+} \equiv \mathbb{1}$, the manifolds $\mathcal{S}^{ \pm}$are trivial, and nothing has to be shown. Therefore, we assume $Q_{+} \neq \mathbb{0}$ and $Q_{+} \neq \mathbb{1}$. In Aulbach \& RAsmussen \& Siegmund [18, Lemma 6.1] (see also RASMUSSEN [143, Lemma 6.3.7], CopPel [54, Chapter 5] and SiEGMUnd [172, Lemma 2.3]), it is shown that there exists a function $T: \mathbb{R} \rightarrow \mathbb{R}^{N \times N}$ of invertible matrices such that the so-called Lyapunov transformation $y=T(t) x$ of system (5.1) leads to the following system with decoupled linearization:

$$
\dot{y}=\underbrace{\left(\begin{array}{cc}
B^{+}(t) & 0 \\
0 & B^{-}(t)
\end{array}\right)}_{=: B(t)} y+\underbrace{T(t) F\left(t, T(t)^{-1} y\right)}_{=: G(t, y)},
$$

where $B^{+}: \mathbb{R} \rightarrow \mathbb{R}^{N^{+} \times N^{+}}$and $B^{-}: \mathbb{R} \rightarrow \mathbb{R}^{N^{-} \times N^{-}}$with $N^{+}:=\operatorname{rk} Q_{+}$and $N^{-}:=\operatorname{rk} Q_{-}$. The transition operators $\Psi^{+}$and $\Psi^{-}$of the linear differential equations $\dot{y}_{+}=B^{+}(t) y_{+}$and $\dot{y}_{-}=B^{-}(t) y_{-}$, respectively, fulfill

$$
\left\|\Psi^{+}(t, s)\right\| \leq 2 K^{2} e^{\alpha(t-s)} \text { for all } t \geq s \quad \text { and } \quad\left\|\Psi^{-}(t, s)\right\| \leq 2 K^{2} e^{\beta(t-s)} \text { for all } t \leq s .
$$


It is also show that

$$
\|T(t)\| \leq \sqrt{2} K \text { and }\left\|T^{-1}(t)\right\| \leq \sqrt{2} \text { for all } t \in \mathbb{R} .
$$

Thus, the Hypothesis on nonlinearity implies the limit relation

$$
\lim _{y \rightarrow 0} \sup _{t \in \mathbb{R}}\left\|D_{2} G(t, y)\right\|=0 \text {. }
$$

We fix a smooth cut-off function $\chi: \mathbb{R}^{N} \rightarrow[0,1]$ (see, e.g., ABRAHAM \& MARSDEN \& RATIU [1, Lemma 4.2.13]) such that

$$
\chi(x)=1 \text { for all } x \in \mathbb{R}^{N} \text { with }\|x\| \leq 1 \quad \text { and } \quad \chi(x)=0 \text { for all } x \in \mathbb{R}^{N} \text { with }\|x\| \geq 2 .
$$

For any $\sigma>0$ with $U_{2 \sigma}(0) \subset U$, we define the function $G_{\sigma}: \mathbb{R} \times \mathbb{R}^{N} \rightarrow \mathbb{R}^{N}$ by

$$
G_{\sigma}(t, x):=\left\{\begin{array}{cc}
\chi\left(\frac{x}{\sigma}\right) G(t, x) & \text { for all } t \in \mathbb{R} \text { and } x \in U \\
0 & \text { for all } t \in \mathbb{R} \text { and } x \notin U
\end{array} .\right.
$$

Due to the mean value inequality (see, e.g., LANG [101, Corollary 4.3, p. 342]), the relation $G(\cdot, 0) \equiv 0$ leads to

$$
\|G(t, x)\| \leq\|x\| \sup _{s \in[0,1]}\left\|D_{2} G(t, s x)\right\| \quad \text { for all } x \in \mathbb{R}^{N} \text { and } t \in \mathbb{R} .
$$

Since $D_{2} G_{\sigma}(t, x)=\chi\left(\frac{x}{\sigma}\right) D_{2} G(t, x)+\frac{1}{\sigma} D \chi\left(\frac{x}{\sigma}\right) G(t, x)$, for all $t \in \mathbb{R}$, we have

$$
\begin{aligned}
& \sup _{x \in \mathbb{R}^{N}}\left\|D_{2} G_{\sigma}(t, x)\right\| \\
\leq & \sup _{\|x\| \leq 2 \sigma}\left\|D_{2} G(t, x)\right\|+\frac{1}{\sigma} \sup _{\|x\| \leq 2} D \chi(x) \sup _{\|x\| \leq 2 \sigma}\left(\|x\| \sup _{s \in[0,1]}\left\|D_{2} G(t, s x)\right\|\right) .
\end{aligned}
$$

Hence,

$$
\sup _{(t, x) \in \mathbb{R} \times \mathbb{R}^{N}}\left\|D_{2} G_{\sigma}(t, x)\right\| \leq\left(1+2 \sup _{x \in \mathbb{R}^{N}} D \chi(x)\right) \sup _{\|x\| \leq 2 \sigma, t \in \mathbb{R}}\left\|D_{2} G(t, x)\right\| .
$$

Due to (5.4), this implies

$$
\lim _{\sigma \rightarrow 0} \sup _{(t, x) \in \mathbb{R} \times \mathbb{R}^{N}}\left\|D_{2} G_{\sigma}(t, x)\right\|=0,
$$

and thus, there exists a $\tilde{\rho}>0$ such that

$$
\dot{y}=B(t) y+G_{\tilde{\rho}}(t, y)
$$

fulfills the (global) hypotheses of SiEGMUND [170, Satz 4.16 and Satz 4.30]. Denoting the general solution of (5.5) by $\tilde{\lambda}$, this means that there exist $C^{1}$-functions $\tilde{s}^{ \pm}: \mathbb{R} \times \mathbb{R}^{N} \rightarrow \mathbb{R}^{N}$ fulfilling

$$
\tilde{s}^{+}(t, \xi)=\tilde{s}^{+}\left(t,\left(\xi_{1}, \ldots, \xi_{N^{+}}, 0, \ldots, 0\right)\right) \in\{(0, \ldots, 0)\} \times \mathbb{R}^{N^{-}} \subset \mathbb{R}^{N}
$$

and

$$
\tilde{s}^{-}(t, \xi)=\tilde{s}^{-}\left(t,\left(0, \ldots, 0, \xi_{N^{+}+1}, \ldots, \xi_{N}\right)\right) \in \mathbb{R}^{N^{+}} \times\{(0, \ldots, 0)\} \subset \mathbb{R}^{N}
$$


for all $t \in \mathbb{R}$ and $\xi \in \mathbb{R}^{N}$ such that the graphs

$$
\tilde{\mathcal{S}}^{+}:=\left\{\left(\tau, \xi+s^{+}(\tau, \xi)\right) \in \mathbb{R} \times \mathbb{R}^{N}: \xi_{i}=0 \text { for } i>N^{+}\right\}
$$

and

$$
\tilde{\mathcal{S}}^{-}:=\left\{\left(\tau, \xi+s^{-}(\tau, \xi)\right) \in \mathbb{R} \times \mathbb{R}^{N}: \xi_{i}=0 \text { for } i \leq N^{+}\right\}
$$

are global nonautonomous invariant manifolds with

$$
\tilde{\mathcal{S}}^{ \pm}:=\left\{(\tau, \xi) \in \mathbb{R} \times \mathbb{R}^{N}: \tilde{\lambda}(\cdot, \tau, \xi) \text { is }\left(\frac{\alpha+\beta}{2}\right)^{ \pm} \text {-quasibounded }\right\} .
$$

We define $\hat{s}^{ \pm}: \mathbb{R} \times \mathbb{R}^{N} \rightarrow \mathbb{R}^{N}$ by

$$
\hat{s}^{ \pm}(t, x):=T(t)^{-1} \tilde{s}^{ \pm}(t, T(t) x) \quad \text { for all } t \in \mathbb{R} \text { and } x \in \mathbb{R}^{N} .
$$

Then, $\hat{s}^{ \pm}$leads to the nonautonomous sets $\hat{\mathcal{S}}^{ \pm}$, which also can be defined by

$$
\hat{\mathcal{S}}^{ \pm}(t):=T(t)^{-1} \tilde{\mathcal{S}}^{ \pm}(t) \text { for all } t \in \mathbb{R} .
$$

Let $\hat{\lambda}$ denote the general solution of the system

$$
\dot{x}=A(t) x+T(t)^{-1} G_{\sigma}(t, T(t) x),
$$

which is obtained via the transformation $x=T(t)^{-1} y$ from system (5.5). Then, the representation

$$
\hat{\mathcal{S}}^{ \pm}:=\left\{(\tau, \xi) \in \mathbb{R} \times \mathbb{R}^{N}: \hat{\lambda}(\cdot, \tau, \xi) \text { is }\left(\frac{\alpha+\beta}{2}\right)^{ \pm} \text {-quasibounded }\right\}
$$

is fulfilled (see RASMUSSEN [143, Satz 6.3.8] or Aulbach \& RASMUSSEN \& SiEgmund [18]), and due to Aulbach \& WANNER [21, p. 83-84, formulae (69), (70)] and (5.3), there exists an $M_{1} \geq 1$ with

$$
\|\hat{\lambda}(t, \tau, \xi)\| \leq M_{1}\|\xi\| e^{\frac{\alpha+\beta}{2}(t-\tau)} \quad \text { for all } t \geq \tau \text { and } \xi \in \hat{\mathcal{S}}^{+}(\tau)
$$

and

$$
\|\hat{\lambda}(t, \tau, \xi)\| \leq M_{1}\|\xi\| e^{\frac{\alpha+\beta}{2}(t-\tau)} \quad \text { for all } t \leq \tau \text { and } \xi \in \hat{\mathcal{S}}^{-}(\tau) .
$$

Because of (5.3),

there exists a $\hat{\rho}>0$ such that the systems (5.7) and (5.1)

$$
\text { coincide on } t \in \mathbb{R} \text { and } x \in U_{\hat{\rho}}(0) \text {. }
$$

Moreover, there exists an $M_{2}>0$ such that $\left\|\hat{s}^{ \pm}(t, x)\right\| \leq M_{2}\|x\|$ for all $t \in \mathbb{R}$ and $x \in \mathbb{R}^{N}$ (see the definition of $\hat{s}^{ \pm},(5.3)$ and Siegmund [170, Satz 4.16 (c)]). This implies the existence of a $\rho>0$ such that with the functions

$$
s^{ \pm}: \mathbb{R} \times U_{\rho}(0) \rightarrow \mathbb{R}^{N}, \quad s^{ \pm}(t, x):=\hat{s}^{ \pm}(t, x) \quad \text { for all } t \in \mathbb{R} \text { and } x \in U_{\rho}(0),
$$

the sets

$$
\mathcal{S}^{ \pm}:=\left\{\left(\tau, \xi+s^{ \pm}(\tau, \xi)\right) \in \mathbb{R} \times \mathbb{R}^{N}: \xi \in \mathcal{R}\left(Q_{ \pm}(\tau)\right) \cap U_{\rho}(0)\right\}
$$


are subsets of $\mathbb{R} \times U_{\hat{\rho}}(0)$. Furthermore, $\mathcal{S}^{ \pm}$are local nonautonomous invariant manifolds of (5.1), since the conditions of Definition 5.1.1 are easily verified $\left(\tilde{\mathcal{S}}^{ \pm}\right.$are global nonautonomous invariant manifolds, and (5.9), (5.6) and (5.3) are fulfilled). For further reference, please note that (5.8) and (5.9) imply

$$
\|\lambda(t, \tau, \xi)\| \leq M_{1}\|\xi\| e^{\frac{\alpha+\beta}{2}(t-\tau)} \quad \begin{array}{ll}
\text { for all } t \geq \tau \text { and } \xi \in \mathcal{S}^{+}(\tau) \text { such that } \\
& \lambda(\tau+c(t-\tau), \tau, \xi) \in U_{\hat{\rho}}(0) \text { for all } c \in[0,1] .
\end{array}
$$

STEP 2. The statements (i)-(iv) are fulfilled.

(i) Suppose that $\alpha<0$, and choose $\varepsilon>0$ arbitrarily. W.l.o.g., assume that $\alpha+\varepsilon<0$. By applying Step 1 with the constants $\alpha$ and $\min \{\beta, \alpha+2 \varepsilon\}$ instead of $\alpha$ and $\beta$, we get another local nonautonomous invariant manifold $\overline{\mathcal{S}}^{+}$, obtained as graph of a function $\bar{s}^{+}: \mathbb{R} \times U_{\bar{\rho}}(0) \rightarrow \mathbb{R}^{N}$ with $0<\bar{\rho}<\rho$. Then, because of $\frac{1}{2}(\alpha+\alpha+2 \varepsilon)=\alpha+\varepsilon$, (5.10) reads as

$$
\|\lambda(t, \tau, \xi)\| \leq M_{1}\|\xi\| e^{\min \left\{\frac{\alpha+\beta}{2}, \alpha+\varepsilon\right\}(t-\tau)} \quad \begin{array}{ll}
\text { for all } t \geq \tau \text { and } \xi \in \overline{\mathcal{S}}^{+}(\tau) \text { such that } \\
& \lambda(\tau+c(t-\tau), \tau, \xi) \in U_{\hat{\rho}}(0) \text { for all } c \in[0,1]
\end{array}
$$

Due to $\alpha+\varepsilon<0$, this means that there exists an $r>0$ such that

$$
\begin{gathered}
\lambda(t, \tau, \xi) \in U_{\rho}(0) \underset{\operatorname{and}}{\text { and }} \\
\|\lambda(t, \tau, \xi)\| \leq M_{1}\|\xi\| e^{\min \left\{\frac{\alpha+\beta}{2}, \alpha+\varepsilon\right\}(t-\tau)} \quad \text { for all }(\tau, \xi) \in \overline{\mathcal{S}}^{+} \cap\left(\mathbb{R} \times U_{r}(0)\right) \text { and } t \geq \tau .
\end{gathered}
$$

Thus, for $(\tau, \xi) \in \overline{\mathcal{S}}^{+} \cap\left(\mathbb{R} \times U_{r}(0)\right)$, the solution $\lambda(\cdot, \tau, \xi)$ is both $\left(\frac{\alpha+\beta}{2}\right)^{+}$-quasibounded and $(\alpha+\varepsilon)^{+}$-quasibounded. From the $\left(\frac{\alpha+\beta}{2}\right)^{+}$-quasiboundedness, we get

$$
\overline{\mathcal{S}}^{+} \cap\left(\mathbb{R} \times U_{r}(0)\right)=\mathcal{S}^{+} \cap\left(\mathbb{R} \times U_{r}(0)\right)
$$

from the dynamic characterization (of the global manifolds) in Step 1. Thus, the proof of (i) is finished.

(ii) can be shown analogously to (i).

(iii) We choose an $L>0$ such that $\alpha+K L<0$. Let $\chi$ denote the cut-off function from Step 1 . Then, we define for any $\sigma>0$ with $U_{2 \sigma}(0) \subset U$ the function $F_{\sigma}: \mathbb{R} \times \mathbb{R}^{N} \rightarrow \mathbb{R}^{N}$ by

$$
F_{\sigma}(t, x):=\left\{\begin{array}{cl}
\chi\left(\frac{x}{\sigma}\right) F(t, x) & \text { for all } t \in \mathbb{R} \text { and } x \in U \\
0 & \text { for all } t \in \mathbb{R} \text { and } x \notin U
\end{array} .\right.
$$

Analogously to Step 1, the relation

$$
\lim _{\sigma \rightarrow 0} \sup _{(t, x) \in \mathbb{R} \times \mathbb{R}^{N}}\left\|D_{2} F_{\sigma}(t, x)\right\|=0
$$

follows, and the limit behavior only depends on $\Gamma$ and $\chi$. This means that there exists an $\tilde{r}=\tilde{r}(\alpha, K, \Gamma, \chi)>0$ such that

$$
\dot{y}=A(t) y+F_{\tilde{r}}(t, y)
$$

fulfills the hypotheses of AULBACH \& WANNER [21, Lemma 3.4, p. 70] with the constants $\alpha, K$ and $L$. We denote the general solution of (5.11) by $\tilde{\lambda}$. Then, due to [21, Lemma 3.4, p. 70], we obtain

$$
\|\tilde{\lambda}(t, \tau, \xi)\| \leq K\|\xi\| e^{\frac{\alpha+K L}{2}(t-\tau)} \quad \text { for all } t \geq \tau \text { and } \xi \in \mathbb{R}^{N} .
$$

We define $r:=\frac{\tilde{r}}{K}$. Since (5.11) coincides with (5.1) on $\mathbb{R} \times U_{\tilde{r}}(0)$, we get

$$
\|\lambda(t, \tau, \xi)\| \leq K\|\xi\| e^{\frac{\alpha+K L}{2}(t-\tau)} \quad \text { for all } t \geq \tau \text { and } \xi \in U_{r}(0) .
$$


This implies the assertion.

(iv) can be proved similarly to (iii) using Lemma 3.7 of AULBACH \& WANNER [21] instead of Lemma 3.4.

5.1.4 REMARK. An alternative way for the construction of nonautonomous invariant manifolds for the ODE (5.1) without applying the Lyapunov transformation as in Step 1 of the preceding proof can be found in PÖTZSCHE [137].

By applying the preceding theorem, in the next two theorems, the existence of past and future invariant manifolds is proved.

5.1.5 TheOREM (PAST INVARIANT MANIFOLDS). In case $\mathbb{\square}=\mathbb{R}_{\mathcal{K}}^{-}$, there exist $\rho>0$ and $C^{1}$-functions $s^{ \pm}: \llbracket \times U_{\rho}(0) \rightarrow \mathbb{R}^{N}$ such that the graphs

$$
\mathcal{S}^{ \pm}:=\left\{\left(\tau, \xi+s^{ \pm}(\tau, \xi)\right) \in \mathbb{\square} \times \mathbb{R}^{N}: \xi \in \mathcal{R}\left(Q_{ \pm}(\tau)\right) \cap U_{\rho}(0)\right\}
$$

are local nonautonomous invariant manifolds. Furthermore, the following statements are fulfilled:

(i) Case $\alpha<0$ and rk $Q_{+} \geq 1$ (Trivial solution is not past repulsive). For all $\varepsilon>0$, there exist $r>0$ and $M \geq 1$ such that for all $(\tau, \xi) \in \mathcal{S}^{+}$with $\|\xi\|<r$, we have

$$
\lambda(t, \tau, \xi) \in U_{\rho}(0) \text { and }\|\lambda(t, \tau, \xi)\| \leq M e^{(\alpha+\varepsilon)(t-\tau)}\|\xi\| \quad \text { for all } \kappa \geq t \geq \tau
$$

(ii) Case $\beta>0$ and rk $Q_{-} \geq 1$ (Trivial solution is not past attractive). For all $\varepsilon>0$, there exists an $r>0$ such that for all $(\tau, \xi) \in \mathcal{S}^{-}$with $\|\xi\|<r$, we have

$$
\lambda(t, \tau, \xi) \in U_{\rho}(0) \text { for all } t \leq \tau \leq \kappa \text { and } \lambda(\cdot, \tau, \xi) \text { is }(\beta-\varepsilon)^{-} \text {-quasibounded. }
$$

(iii) Case $\alpha<0$ and $Q_{+} \equiv \mathbb{1}$ (Trivial solution is past attractive). There exists an $r=r(\alpha, K, \Gamma)>0$ with

$$
\lim _{t \rightarrow \infty} \sup _{\tau \in \rrbracket} d\left(\lambda\left(\tau, \tau-t, U_{r}(0)\right) \mid\{0\}\right)=0
$$

(iv) Case $\beta>0$ and $Q_{-} \equiv \mathbb{1}$ (Trivial solution is past repulsive). There exists an $r=r(\beta, K, \Gamma)>0$ with

$$
\lim _{t \rightarrow \infty} \sup _{\tau \in \llbracket} d\left(\lambda\left(\tau-t, \tau, U_{r}(0)\right) \mid\{0\}\right)=0
$$

PROOF. We first observe that all assertions of Theorem 5.1.3 also hold in case (5.1) is a differential equation of Carathéodory type, since equations of this form are treated in SIEGMUND [170] and AUlBACH \& WANNER [22]. This is important, because we want to apply this theorem to the Carathéodory differential equation

$$
\dot{x}=B(t) x+G(t, x)
$$

with functions $B: \mathbb{R} \rightarrow \mathbb{R}^{N \times N}$ and $G: \mathbb{R} \times U \rightarrow \mathbb{R}^{N}$ defined as follows. Let $C \in \mathbb{R}^{N \times N}$ be the matrix fulfilling

$$
C x=\alpha x \quad \text { for all } x \in \mathcal{R}\left(Q_{+}(\kappa)\right) \quad \text { and } \quad C x=\beta x \quad \text { for all } x \in \mathcal{R}\left(Q_{-}(\kappa)\right) .
$$


Then, we define the functions $B$ and $G$ by

$$
B(t):=\left\{\begin{array}{cl}
A(t) & \text { for all } t \leq \kappa \\
C & \text { for all } t>\kappa
\end{array} \quad \text { and } \quad G(t, x):=\left\{\begin{array}{cl}
F(t, x) & \text { for all } t \leq \kappa, x \in U \\
0 & \text { for all } t>\kappa, x \in U
\end{array}\right.\right.
$$

It is easy to see that equation (5.12) fulfills the hypotheses of Theorem 5.1.3 with the invariant projector $\hat{Q}_{+}: \mathbb{R} \rightarrow \mathbb{R}^{N \times N}$ defined by

$$
\hat{Q}_{+}:=\left\{\begin{array}{ll}
Q_{+}(t) & \text { for all } t \leq \kappa \\
Q_{+}(\kappa) & \text { for all } t>\kappa
\end{array} .\right.
$$

Then, there exist nonautonomous invariant manifolds of (5.12) which, by restriction to $\mathbb{R}_{\kappa}^{-} \times \mathbb{R}^{N}$, are nonautonomous invariant manifolds of (5.1). The statements (i)-(iv) follow directly.

An analogous statement is fulfilled by considering $\mathbb{R}_{\kappa}^{+}$instead of $\mathbb{R}_{\mathcal{\kappa}}^{-}$.

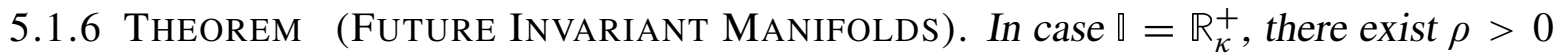
and $C^{1}$-functions $s^{ \pm}: \llbracket \times U_{\rho}(0) \rightarrow \mathbb{R}^{N}$ such that the graphs

$$
\mathcal{S}^{ \pm}:=\left\{\left(\tau, \xi+s^{ \pm}(\tau, \xi)\right) \in \mathbb{\square} \times \mathbb{R}^{N}: \xi \in \mathcal{R}\left(Q_{ \pm}(\tau)\right) \cap U_{\rho}(0)\right\}
$$

are local nonautonomous invariant manifolds. Furthermore, the following statements are fulfilled:

(i) Case $\alpha<0$ and rk $Q_{+} \geq 1$ (Trivial solution is not future repulsive). For all $\varepsilon>0$, there exists an $r>0$ such that for all $(\tau, \xi) \in \mathcal{S}^{+}$with $\|\xi\|<r$, we have

$$
\lambda(t, \tau, \xi) \in U_{\rho}(0) \text { for all } t \geq \tau \geq \kappa \text { and } \lambda(\cdot, \tau, \xi) \text { is }(\alpha+\varepsilon)^{+} \text {-quasibounded. }
$$

(ii) Case $\beta>0$ and rk $Q_{-} \geq 1$ (Trivial solution is not future attractive). For all $\varepsilon>0$, there exist $r>0$ and $M \geq 1$ such that for all $(\tau, \xi) \in \mathcal{S}^{-}$with $\|\xi\|<r$, we have

$$
\lambda(t, \tau, \xi) \in U_{\rho}(0) \text { and }\|\lambda(t, \tau, \xi)\| \leq M e^{(\beta-\varepsilon)(t-\tau)}\|\xi\| \quad \text { for all } \kappa \leq t \leq \tau .
$$

(iii) Case $\alpha<0$ and $Q_{+} \equiv \mathbb{1}$ (Trivial solution is future attractive). There exists an $r=r(\alpha, K, \Gamma)>0$ with

$$
\lim _{t \rightarrow \infty} \sup _{\tau \in \mathbb{1}} d\left(\lambda\left(\tau+t, \tau, U_{r}(0)\right) \mid\{0\}\right)=0 .
$$

(iv) Case $\beta>0$ and $Q_{-} \equiv \mathbb{1}$ (Trivial solution is future repulsive). There exists an $r=r(\beta, K, \Gamma)>0$ with

$$
\lim _{t \rightarrow \infty} \sup _{\tau \in \mathbb{1}} d\left(\lambda\left(\tau, \tau+t, U_{r}(0)\right) \mid\{0\}\right)=0 .
$$

PROOF. See proof of Theorem 5.1.5.

\subsubsection{REMARKS.}

(i) The sets $\mathcal{S}^{+}$and $\mathcal{S}^{-}$of the above theorems are also denoted as all-time (past, future, respectively) pseudo-stable and pseudo-unstable invariant manifolds of (5.1), respectively. To be 
more specific, $\mathcal{S}^{+}$describes an all-time (past, future, respectively)

$$
\left.\begin{array}{c}
\text { center-stable } \\
\text { stable } \\
\text { strongly stable }
\end{array}\right\} \text { invariant manifold in case }\left\{\begin{array}{c}
\beta>0 \\
\alpha<0<\beta \\
\beta<0
\end{array} .\right.
$$

Accordingly, $\mathcal{S}^{-}$describes an all-time (past, future, respectively)

$$
\left.\begin{array}{c}
\text { center-unstable } \\
\text { unstable } \\
\text { strongly unstable }
\end{array}\right\} \text { invariant manifold in case }\left\{\begin{array}{c}
\alpha<0 \\
\alpha<0<\beta \\
\alpha>0
\end{array} .\right.
$$

This terminology corresponds to the autonomous situation of invariant manifolds considered, e.g., in CHOW \& LI \& WANG [46]. Center manifolds are obtained as intersections of center-stable and center-unstable invariant manifolds.

(ii) In the hyperbolic situation $(\alpha<0<\beta)$, the all-time invariant manifolds $\mathcal{S}^{ \pm}$of Theorem 5.1.3 are uniquely determined. Easy examples (see, e.g., HALE \& KoçAK [77, Example 10.13, p. 322]), however, show that center-stable, center-unstable or center manifolds are nonunique in general. Since global invariant manifolds are uniquely determined, different cut-off-techniques (as used in the proof of Theorem 5.1.3) lead to different manifolds. In the situation of Theorem 5.1.5 and Theorem 5.1.6, the question of nonuniqueness is more subtle. In the hyperbolic case, only the pseudo-unstable manifold $\mathcal{S}^{-}$of Theorem 5.1.5 and the pseudo-stable manifold $\mathcal{S}^{+}$of Theorem 5.1.6 are uniquely determined. This corresponds to Remark 4.1.12 in the linear situation.

\subsection{An Application to Bifurcation TheOry}

In autonomous bifurcation theory, it is necessary that at least one eigenvalue of the linearization in a bifurcating equilibrium crosses the imaginary axis. In this section, this fact is generalized with respect to the notions of past, future and all-time bifurcation. For a similar result in the context of random dynamical systems (concerning the Lyapunov exponents of ergodic invariant measures), we refer to ARNOLD \& XU [9] and ARNOLD [5, Theorem 9.2.3, p. 471].

Let $\llbracket$ be an unbounded interval of the form $\mathbb{R}_{\kappa}^{-}, \mathbb{R}_{\kappa}^{+}$or $\mathbb{R}$, respectively. In this section, we consider nonlinear differential equations of the form

$$
\dot{x}=A(t, \alpha) x+F(t, x, \alpha)
$$

with a continuous matrix-valued function $A: \llbracket \times\left(\alpha_{-}, \alpha_{+}\right) \rightarrow \mathbb{R}^{N \times N}$ and a $C^{1}$-function $F: \mathbb{\square} \times U \times\left(\alpha_{-}, \alpha_{+}\right) \rightarrow \mathbb{R}^{N}$, where $U$ is supposed to be a neighborhood of 0 . Furthermore, we assume that $F(t, 0, \alpha)=0$ for all $\alpha \in\left(\alpha_{-}, \alpha_{+}\right)$und $t \in \mathbb{\square}$.

5.2.1 TheOREM (LinEARIZATION AND BIfURCATION). We suppose that the trivial solution of (5.13) $\alpha$ admits a past (future, all-time, respectively) supercritical bifurcation at the parameter value $\alpha_{0} \in\left(\alpha_{-}, \alpha_{+}\right)$and there exists an $\hat{\alpha}>\alpha_{0}$ with

$$
\lim _{x \rightarrow 0} \sup _{t \in \llbracket, \alpha \in\left[\alpha_{0}, \hat{\alpha}\right]}\left\|D_{2} F(t, x, \alpha)\right\|=0
$$

and 


$$
\lim _{\alpha \searrow \alpha_{0}} \sup _{t \in \mathbb{\prod}}\left\|A(t, \alpha)-A\left(t, \alpha_{0}\right)\right\|=0 .
$$

Then, we have

$$
0 \in \Sigma_{A\left(\cdot, \alpha_{0}\right)}^{\leftarrow}, \Sigma_{A\left(\cdot, \alpha_{0}\right)}, \Sigma_{A\left(\cdot, \alpha_{0}\right)}, \text { respectively }
$$

An analogous statement is fulfilled in case of a subcritical bifurcation.

PROOF. We only treat the case of an all-time bifurcation, since the other proofs are similar. Arguing negatively, we suppose that zero is not contained in $\Sigma \overleftrightarrow{A\left(\cdot, \alpha_{0}\right)}$. We distinguish the following two cases.

CASE 1. There exists an $\tilde{\alpha} \in\left(\alpha_{0}, \hat{\alpha}\right)$ such that the trivial solution of $(5.13)_{\alpha}$ is all-time attractive for all $\alpha \in\left(\alpha_{0}, \tilde{\alpha}\right)$.

First, assume that $\Sigma_{A\left(\cdot, \alpha_{0}\right)}^{\leftrightarrow} \cap(0, \infty] \neq \emptyset$. Since $0 \notin \Sigma_{A\left(\cdot, \alpha_{0}\right)}^{\leftrightarrow}$, this means that the linear differential equation $\dot{x}=A\left(t, \alpha_{0}\right) x$ admits a nonhyperbolic all-time exponential dichotomy with growth rate $\gamma>0$ and an invariant projector $Q_{\alpha_{0}}$ such that rk $Q_{\alpha_{0}}<N$ (please note that due to Theorem 4.2.9, the all-time spectrum is closed). Due to Theorem 4.5.1 and (5.15), there exists an $\alpha_{1}>\alpha_{0}$ such that for all $\alpha \in\left(\alpha_{0}, \alpha_{1}\right)$, the linear differential equation

$$
\dot{x}=A(t, \alpha) x
$$

admits a nonhyperbolic all-time exponential dichotomy with growth rate $\gamma$ and an invariant projector $Q_{\alpha}$ such that rk $Q_{\alpha}<N$. Hence,

$$
\Sigma_{A(\cdot, \alpha)}^{\leftrightarrow} \cap(\gamma, \infty] \neq \emptyset \quad \text { for all } \alpha \in\left(\alpha_{0}, \alpha_{1}\right) .
$$

This means that at least one spectral interval of $\Sigma_{A(\cdot, \alpha)}, \alpha \in\left(\alpha_{0}, \alpha_{1}\right)$, lies in $(\gamma, \infty]$, and hence, Theorem 5.1 .3 (ii) is applicable with $\beta=\gamma>0$ and rk $Q_{-}=N-\operatorname{rk} Q_{\alpha} \geq 1$. This implies that the trivial solution of $(5.13)_{\alpha}$ is not all-time attractive, which is a contradiction to the hypothesis of Case 1 , and thus, there exists a $\delta<0$ with

$$
\Sigma_{A\left(\cdot, \alpha_{0}\right)}^{\leftrightarrow} \subset[-\infty, \delta)
$$

(again, we use the fact that all-time dichotomy spectra are closed). Because of Theorem 4.5.1 and (5.15) (cf. the argumentation above), there exists an $\alpha_{2} \in\left(\alpha_{0}, \alpha_{1}\right)$ with

$$
\Sigma_{A(\cdot, \alpha)}^{\leftrightarrow} \subset[-\infty, \delta] \text { for all } \alpha \in\left[\alpha_{0}, \alpha_{2}\right]
$$

We apply Theorem 5.1 .3 (iii) and obtain that, since due to $(5.14)$, the function $\Gamma:(0,1) \rightarrow \overline{\mathbb{R}}^{+}$ can be chosen independently of $\alpha$, the lower bound $r$ of this theorem for the radius of all-time attraction $\mathfrak{A}_{0}^{\leftrightarrow}$ is also independent of $\alpha$. Hence, $\mathfrak{A}_{0}^{\leftrightarrow}$ does not converge to zero in the limit $\alpha \triangleleft \alpha_{0}$. This contradiction finishes the proof of this case.

CASE 2. There exists an $\tilde{\alpha} \in\left(\alpha_{0}, \hat{\alpha}\right)$ such that the trivial solution of $(5.13)_{\alpha}$ is all-time repulsive for all $\alpha \in\left(\alpha_{0}, \tilde{\alpha}\right)$.

This case is treated analogously to Case 1 .

\subsection{LINEARIZED ATTRACTIVITY AND REPULSIVITY}

In Section 5.1, properties of attractivity and repulsivity for a nonlinear system have been derived already by studying the linearization. In contrast to these considerations, in this section, more 
quantitative results are obtained, and furthermore, $C^{1}$-differentiability is not assumed but only continuity.

We first concentrate on the notions of past, future and all-time attractivity and repulsivity.

5.3.1 TheOREM (LineARIZED ATtRACTIVITy AND RePUlsivity, PART I). Consider an unbounded interval $\square$ of the form $\mathbb{R}_{\kappa}^{-}, \mathbb{R}_{\kappa}^{+}$or $\mathbb{R}$, respectively, and let

$$
\dot{x}=A(t) x+F(t, x)
$$

be a nonautonomous differential equation with continuous functions $A: \mathbb{\rightarrow} \rightarrow \mathbb{R}^{N \times N}$ and

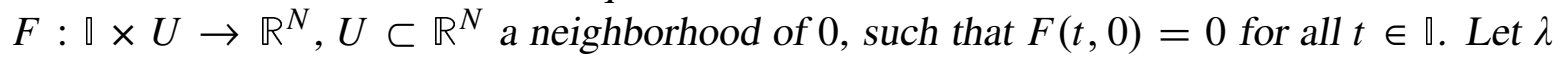
denote the general solution of (5.16) and $\Lambda: \square \times \square \rightarrow \mathbb{R}^{N \times N}$ denote the transition operator of the linearized equation $\dot{x}=A(t) x$. Then, the following statements are fulfilled:

(i) In case there exist $\beta<0, K \geq 1$ and $\delta>0$ such that

$$
\|\Lambda(t, s)\| \leq K e^{\beta(t-s)} \quad \text { for all } t \geq s
$$

and

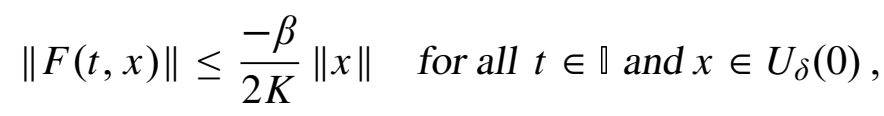

we have

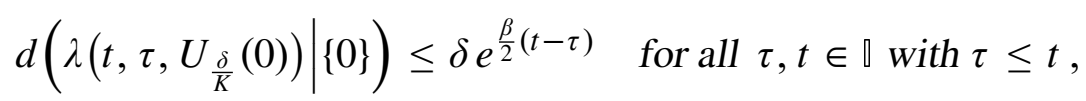

i.e., the trivial solution of (5.16) is past (future, all-time, respectively) attractive.

(ii) In case there exist $\beta>0, K \geq 1$ and $\delta>0$ such that

$$
\|\Lambda(t, s)\| \leq K e^{\beta(t-s)} \quad \text { for all } t \leq s
$$

and

$$
\|F(t, x)\| \leq \frac{\beta}{2 K}\|x\| \quad \text { for all } t \in \llbracket \text { and } x \in U_{\delta}(0),
$$

we have

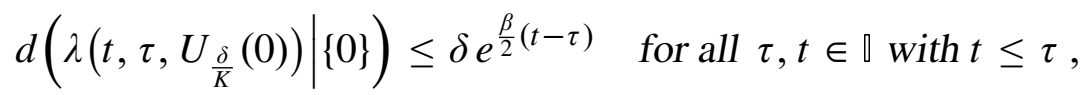

i.e., the trivial solution of (5.16) is past (future, all-time, respectively) repulsive.

PROOF. We only prove (i), since (ii) can be shown analogously. Given $\tau \in \llbracket$ and $\xi \in U_{\delta}(0)$, we now prove an estimate for the general solution under the additional assumption

$$
\lambda(t, \tau, \xi) \in U_{\delta}(0) \text { for all } t \geq \tau .
$$

The solution $\lambda(\cdot, \tau, \xi)$ of (5.16) is also a solution of inhomogeneous linear differential equation

$$
\dot{x}=A(t) x+F(t, \lambda(t, \tau, \xi)) .
$$

Thus, the variation of constants formula (Proposition A.1.6) implies

$$
\lambda(t, \tau, \xi)=\Lambda(t, \tau) \xi+\int_{\tau}^{t} \Lambda(t, s) F(s, \lambda(s, \tau, \xi)) d s \quad \text { for all } t \geq \tau,
$$


and hence,

$$
\begin{aligned}
\|\lambda(t, \tau, \xi)\| & \leq\|\Lambda(t, \tau)\|\|\xi\|+\int_{\tau}^{t}\|\Lambda(t, s)\|\|F(s, \lambda(s, \tau, \xi))\| d s \\
& \stackrel{(5.17)}{\leq} K e^{\beta(t-\tau)}\|\xi\|+\int_{\tau}^{t} K e^{\beta(t-s)} \frac{-\beta}{2 K}\|\lambda(s, \tau, \xi)\| d s \quad \text { for all } t \geq \tau
\end{aligned}
$$

is fulfilled. This implies

$$
e^{-\beta t}\|\lambda(t, \tau, \xi)\| \leq K e^{-\beta \tau}\|\xi\|+\frac{-\beta}{2} \int_{\tau}^{t} e^{-\beta s}\|\lambda(s, \tau, \xi)\| d s \quad \text { for all } t \geq \tau .
$$

Hence, Gronwall's inequality (cf. Lemma A.2.1) yields the estimate

$$
\|\lambda(t, \tau, \xi)\| \leq K e^{\frac{\beta}{2}(t-\tau)}\|\xi\| \quad \text { for all } t \geq \tau .
$$

We define $\eta:=\frac{\delta}{K}$. Since $\frac{\beta}{2}<0$, for all $\tau \in \mathbb{Q}$ and $\xi \in U_{\eta}(0)$, the assumption (5.19) is fulfilled, therefore, (5.20) holds for such $\tau$ and $\xi$. This implies

$$
d\left(\lambda\left(t, \tau, U_{\eta}(0)\right) \mid\{0\}\right) \leq K \eta e^{\frac{\beta}{2}(t-\tau)} \quad \text { for all } \tau, t \in \mathbb{\text { with }} \tau \leq t .
$$

From this inequality, the required conditions for the past (future, all-time, respectively) attractivity are easily obtained.

In case of finite-time attractivity and repulsivity, the following result is obtained.

5.3.2 Theorem (Linearized AtTractivity AND Repulsivity, PART II). Consider a compact interval $\square:=[\tau, \tau+T]$ for some $\tau \in \mathbb{R}$ and $T>0$, and let

$$
\dot{x}=A(t) x+F(t, x)
$$

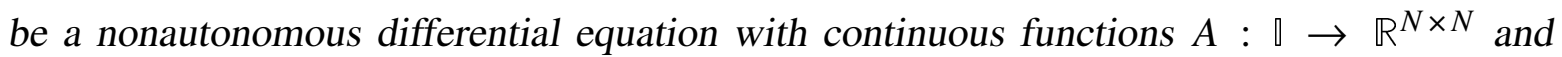
$F: \llbracket \times U \rightarrow \mathbb{R}^{N}, U \subset \mathbb{R}^{N}$ a neighborhood of 0 , such that $F(t, 0)=0$ for all $t \in \mathbb{\square}$. Let $\lambda$ denote the general solution of (5.21) and $\Lambda: \square \times \square \rightarrow \mathbb{R}^{N \times N}$ denote the transition operator of the linearized equation $\dot{x}=A(t) x$, and define

$$
K_{+}:=\sup \{\|\Lambda(t, s)\|: \tau \leq s \leq t \leq \tau+T\}
$$

and

$$
K_{-}:=\sup \{\|\Lambda(t, s)\|: \tau \leq t \leq s \leq \tau+T\} .
$$

Then, the following statements are fulfilled:

(i) In case

$$
\|\Lambda(\tau+T, \tau)\|<1
$$

and there exist $\delta>0$ and $\beta>1$ with

$$
\|F(t, x)\| \leq-\frac{\ln (\beta\|\Lambda(\tau+T, \tau)\|)}{T K_{+}}\|x\| \quad \text { for all } t \in \mathbb{Q} \text { and } x \in U_{\delta}(0),
$$

there exists an $\eta>0$ such that

$$
\|\lambda(\tau+T, \tau, \xi)\| \leq \beta^{-1}\|\xi\| \quad \text { for all } \xi \in U_{\eta}(0),
$$


i.e., the trivial solution of $(5.21)$ is $(\tau, T)$-attractive.

(ii) In case

$$
\|\Lambda(\tau, \tau+T)\|<1
$$

and there exist $\delta>0$ and $\beta>1$ with

$$
\|F(t, x)\| \leq-\frac{\ln (\beta\|\Lambda(\tau, \tau+T)\|)}{T K_{-}}\|x\| \quad \text { for all } t \in \mathbb{a} \text { and } x \in U_{\delta}(0),
$$

there exists an $\eta>0$ such that

$$
\|\lambda(\tau, \tau+T, \xi)\| \leq \beta^{-1}\|\xi\| \quad \text { for all } \xi \in U_{\eta}(0),
$$

i.e., the trivial solution of $(5.21)$ is $(\tau, T)$-repulsive.

ProOF. We only prove (i), since (ii) can be shown analogously. Due to the continuity of the general solution (cf. Proposition A.1.3), there exists an $\eta<\delta$ with

$$
\|\lambda(t, \tau, \xi)\|<\delta \text { for all } t \in \llbracket \text { and } \xi \in U_{\eta}(0) .
$$

We choose $\xi \in U_{\eta}(0)$ arbitrarily. Then, the solution $\lambda(\cdot, \tau, \xi)$ of (5.21) is also a solution of the linear differential equation

$$
\dot{x}=A(t) x+F(t, \lambda(t, \tau, \xi)) .
$$

Thus, the variation of the constants formula (cf. Proposition A.1.6) implies

$$
\lambda(t, \tau, \xi)=\Lambda(t, \tau) \xi+\int_{\tau}^{t} \Lambda(t, s) F(s, \lambda(s, \tau, \xi)) d s \quad \text { for all } t \in \mathbb{\square}
$$

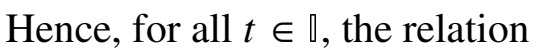

$$
\begin{aligned}
\|\lambda(t, \tau, \xi)\| & \leq\|\Lambda(t, \tau) \xi\|+\int_{\tau}^{t}\|\Lambda(t, s)\|\|F(s, \lambda(s, \tau, \xi))\| d s \\
& \leq\|\Lambda(t, \tau)\|\|\xi\|-K_{+} \frac{\ln (\beta\|\Lambda(\tau+T, \tau)\|)}{T K_{+}} \int_{\tau}^{t}\|\lambda(s, \tau, \xi)\| d s \\
& =\|\Lambda(t, \tau)\|\|\xi\|-\frac{\ln (\beta\|\Lambda(\tau+T, \tau)\|)}{T} \int_{\tau}^{t}\|\lambda(s, \tau, \xi)\| d s .
\end{aligned}
$$

We apply Gronwall's inequality (cf. Lemma A.2.1) and obtain for all $\xi \in U_{\eta}(0)$,

$$
\begin{aligned}
\|\lambda(\tau+T, \tau, \xi)\| & \leq\|\Lambda(\tau+T, \tau)\|\|\xi\| \exp (-\ln (\beta\|\Lambda(\tau+T, \tau)\|)) \\
& =\beta^{-1}\|\xi\| .
\end{aligned}
$$

This finishes the proof of this theorem.

\subsubsection{REMARKS.}

(i) Concerning Theorem 5.3.1 and Theorem 5.3.2, the past (future, all-time, $(\tau, T)$-, respectively) dichotomy spectrum of the linearization $\dot{x}=A(t) x$ is a subset of $\mathbb{R}^{-}$. 
(ii) The conditions (5.17), (5.18) of Theorem 5.3.1 and (5.22), (5.23) of Theorem 5.3.2 are fulfilled if we have

$$
\lim _{x \rightarrow 0} \sup _{t \in \rrbracket} \frac{\|F(t, x)\|}{\|x\|}=0 .
$$

This limit relation is only sufficient but not necessary for the above mentioned conditions.

\subsection{BifurCATION THEORY OF Adiabatic Systems}

In this section, a relationship between the bifurcation theory of adiabatic systems (see, e.g., BEnoîT [27], BERGLUND [29] or LEBOVITZ \& SchAAR $[105,106])$ and the concept of finitetime bifurcation is pointed out.

The bifurcation theory of adiabatic systems is usually called dynamic bifurcation theory (see title of BENOÎT [27]). We will not employ this term here, since it is unfortunately used in a different sense both in autonomous bifurcation theory (as opposed to static bifurcation theory, cf. Subsection 2.4.1) and random bifurcation theory (as opposed to phenomenological bifurcation theory, cf. Subsection 2.4.3).

Let $I$ be an open interval and $D \subset \mathbb{R}^{N}$ be an open set, and consider an autonomous differential equation

$$
\dot{x}=f(\alpha, x) \text {, }
$$

depending on a parameter $\alpha$ with a $C^{1}$-function $f: I \times D \rightarrow \mathbb{R}^{N}$. To mimic the situation of a slowly varying parameter, for $\varepsilon>0$, we also look at the system

$$
\dot{x}=f(\varepsilon t, x) \text {, }
$$

which can be transformed via the slow time $t \mapsto \varepsilon t$ into the so-called adiabatic or singularlyperturbed system

$$
\dot{x}=\frac{1}{\varepsilon} f(t, x) \text {. }
$$

The central question of the bifurcation theory of adiabatic systems is: How do solutions of $(5.25)_{\varepsilon}$ behave in the limit $\varepsilon \succ 0$ in case $(5.24)_{\alpha}$ admits an autonomous bifurcation?

We assume that (5.24) $)_{\alpha}$ admits a bifurcation of the following type.

HYPOTHESIS. For fixed $\alpha_{-}<\alpha_{+} \in I$, we consider two different continuous functions $s_{1}, s_{2}:\left[\alpha_{-}, \alpha_{+}\right] \rightarrow D$ such that

$$
s_{1}\left(\alpha_{-}\right)=s_{2}\left(\alpha_{-}\right) \quad \text { and } \quad f\left(\alpha, s_{1}(\alpha)\right)=f\left(\alpha, s_{2}(\alpha)\right)=0 \text { for all } \alpha \in\left[\alpha_{-}, \alpha_{+}\right] .
$$

We suppose that

- the function $s_{1}$ describes attractive equilibria of (5.24) $)_{\alpha}$, i.e., for $\alpha \in\left(\alpha_{-}, \alpha_{+}\right.$], all eigenvalues of $D_{2} f\left(\alpha, s_{1}(\alpha)\right)$ have a negative real part,

- the function $s_{2}$ describes hyperbolic equilibria of (5.24) $\alpha$, i.e., for $\alpha \in\left(\alpha_{-}, \alpha_{+}\right]$, all eigenvalues of $D_{2} f\left(\alpha, s_{2}(\alpha)\right)$ have a non-vanishing real part.

The existence of such a bifurcation implies that $D_{2} f\left(\alpha_{-}, s_{1}\left(\alpha_{-}\right)\right)$has an eigenvalue with vanishing real part. 
In the bifurcation theory of adiabatic systems, the occurrence of the following two possibilities is discussed:

(a) There exists a family of solutions $v_{\varepsilon}:\left[\alpha_{-}, \alpha_{+}\right] \rightarrow D$ of $(5.25)_{\varepsilon}, \varepsilon>0$ small, which converge to the attractive equilibrium branch in the limit $\varepsilon \rightarrow 0$.

(b) There exists a family of solutions $v_{\varepsilon}:\left[\alpha_{-}, \alpha_{+}\right] \rightarrow D$ of $(5.25)_{\varepsilon}, \varepsilon>0$ small, which follow for some time interval (which does not depend on $\varepsilon$ ) the equilibrium branch $s_{2}$ and then jump to the stable branch $s_{1}$.

The phenomenon (b) is called bifurcation delay or delayed exchange of stabilities. The corresponding solutions are said to be canard solutions. The property (a), which we will discuss in this section, is generalized by the following definition.

5.4.1 Definition (Adiabatic Solutions). Let $\alpha_{0}<\alpha_{1} \in I$. A continuous function $s:\left[\alpha_{0}, \alpha_{1}\right] \rightarrow D$ with

$$
f(\alpha, s(\alpha))=0 \quad \text { for all } \alpha \in\left[\alpha_{0}, \alpha_{1}\right]
$$

is called equilibrium branch which admits adiabatic solutions if there exist $\tilde{\varepsilon}>0$ and a function $v:\left[\alpha_{0}, \alpha_{1}\right] \times(0, \tilde{\varepsilon}) \rightarrow D$ such that $v(\cdot, \varepsilon)$ is a solution of $(5.25)_{\varepsilon}$ and

$$
\lim _{\varepsilon \succ 0} \sup _{\alpha \in\left[\alpha_{0}, \alpha_{1}\right]}\|v(\alpha, \varepsilon)-s(\alpha)\|=0 .
$$

In case the equilibria of (5.24) $\alpha$ described by the function $s$ are hyperbolic, the existence of adiabatic solutions follows from the following theorem. A proof can be found, e.g., in BERGLUND [29, Theorem 5.1, p. 140].

5.4.2 Theorem (Existence of Adiabatic Solutions). Let $\alpha_{0}<\alpha_{1} \in I$, and consider a continuous function $s:\left[\alpha_{0}, \alpha_{1}\right] \rightarrow D$ such that

$$
f(\alpha, s(\alpha))=0 \text { and } D_{2} f(\alpha, s(\alpha)) \text { is hyperbolic for all } \alpha \in\left[\alpha_{0}, \alpha_{1}\right] \text {. }
$$

Then, the equilibrium branch $s$ admits adiabatic solutions.

In the next lemma, linearizations near a branch of stable equilibria are examined.

5.4.3 LEMMA. Let $\alpha_{0}<\alpha_{1} \in I$, and consider a continuous function $s:\left[\alpha_{0}, \alpha_{1}\right] \rightarrow D$ such that $f(\alpha, s(\alpha))=0$ and $D_{2} f(\alpha, s(\alpha))$ has only eigenvalues with negative real part for all $\alpha \in\left[\alpha_{0}, \alpha_{1}\right]$. Then, there exist constants $\delta>0, K \geq 1$ and $\gamma<0$ such that for all continuous functions $h:\left[\alpha_{0}, \alpha_{1}\right] \rightarrow \mathbb{R}^{N}$ with

$$
\|h(\alpha)-s(\alpha)\|<\delta \quad \text { for all } \alpha \in\left[\alpha_{0}, \alpha_{1}\right]
$$

the transition operator $\Lambda_{\varepsilon}$ of the linear system

$$
\dot{x}=\frac{1}{\varepsilon} D_{2} f(t, h(t)) x
$$

fulfills 


$$
\left\|\Lambda_{\varepsilon}\left(\alpha_{1}, \alpha_{0}\right)\right\| \leq K \exp \left(\frac{\gamma}{\varepsilon}\left(\alpha_{1}-\alpha_{0}\right)\right)
$$

Proof. We define $A(\alpha):=D_{2} f(\alpha, s(\alpha))$ for all $\alpha \in\left[\alpha_{0}, \alpha_{1}\right]$. Since $A(\cdot)$ is continuous on the compact interval $\left[\alpha_{0}, \alpha_{1}\right]$ and all eigenvalues of $A(\alpha), \alpha \in\left[\alpha_{0}, \alpha_{1}\right]$, have negative real part, there exist $\tilde{K} \geq 1$ and $\tilde{\gamma}<0$ with

$$
\left\|e^{A(\alpha) t}\right\| \leq \tilde{K} e^{\tilde{\gamma} t} \text { for all } t \geq 0 \text { and } \alpha \in\left[\alpha_{0}, \alpha_{1}\right] .
$$

Due to the uniform continuity of $D_{2} f(\cdot, \cdot)$ on compact sets, there exists a $\delta>0$ such that

$$
\|D_{2} f(\alpha, x)-\underbrace{D_{2} f(\alpha, s(\alpha))}_{A(\alpha)}\|<-\frac{\tilde{\gamma}}{16 \tilde{K}^{2}} \quad \text { for all } \alpha \in\left[\alpha_{0}, \alpha_{1}\right] \text { and } x \in U_{\delta}(s(\alpha)) \text {. }
$$

Since $A(\cdot)$ is uniform continuous on $\left[\alpha_{0}, \alpha_{1}\right]$, there exist $n \in \mathbb{N}$ and constants $\beta_{i} \in\left[\alpha_{0}, \alpha_{1}\right]$, $i \in\{0, \ldots, n\}$, with $\alpha_{0}=\beta_{0}<\beta_{1}<\cdots<\beta_{n}=\alpha_{1}$ such that for all $i \in\{1, \ldots, n\}$, we have

$$
\left\|A\left(\beta_{i-1}\right)-A(\alpha)\right\|<-\frac{\tilde{\gamma}}{16 \tilde{K}^{2}} \quad \text { for all } \alpha \in\left[\beta_{i-1}, \beta_{i}\right] .
$$

Let $h:\left[\alpha_{0}, \alpha_{1}\right] \rightarrow \mathbb{R}^{N}$ be a continuous function fulfilling

$$
\|h(\alpha)-s(\alpha)\|<\delta \quad \text { for all } \alpha \in\left[\alpha_{0}, \alpha_{1}\right]
$$

and consider the linear system

$$
\dot{x}=D_{2} f(\varepsilon t, h(\varepsilon t)) x
$$

for fixed $\varepsilon>0$. The transition operator of (5.28) is denoted by $\Psi_{\varepsilon}$. Due to (5.26) and (5.27), for fixed $i \in\{1, \ldots, n\}$,

$$
\left\|D_{2} f(\varepsilon t, h(\varepsilon t))-A\left(\beta_{i-1}\right)\right\|<-\frac{\tilde{\gamma}}{8 \tilde{K}^{2}} \quad \text { for all } t \in\left[\frac{\beta_{i-1}}{\varepsilon}, \frac{\beta_{i}}{\varepsilon}\right]
$$

is fulfilled. Therefore, Theorem 4.5.1 implies

$$
\left\|\Psi_{\varepsilon}\left(\frac{\beta_{i}}{\varepsilon}, \frac{\beta_{i-1}}{\varepsilon}\right)\right\| \leq \frac{5 \tilde{K}^{2}}{2} \exp \left(\left(\tilde{\gamma}-\frac{\tilde{\gamma}}{2 K}\right) \frac{\beta_{i}-\beta_{i-1}}{\varepsilon}\right) .
$$

Hence, the relation

$$
\left\|\Psi_{\varepsilon}\left(\frac{\alpha_{1}}{\varepsilon}, \frac{\alpha_{0}}{\varepsilon}\right)\right\| \leq\left(\frac{5 \tilde{K}^{2}}{2}\right)^{n} \exp \left(\left(\tilde{\gamma}-\frac{\tilde{\gamma}}{2 K}\right) \frac{\alpha_{1}-\alpha_{0}}{\varepsilon}\right)=: K \exp \left(\frac{\gamma}{\varepsilon}\left(\alpha_{1}-\alpha_{0}\right)\right)
$$

holds. This implies the assertion, since $\Lambda_{\varepsilon}\left(\alpha_{1}, \alpha_{0}\right)=\Psi_{\varepsilon}\left(\frac{\alpha_{1}}{\varepsilon}, \frac{\alpha_{0}}{\varepsilon}\right)$.

Using the preceding lemma, we are able to prove the following relationship between adiabatic solutions of attractive equilibrium branches and the concept of finite-time attractivity. 
5.4.4 Corollary. Let $\alpha_{0}<\alpha_{1} \in I$, and consider an equilibrium branch $s:\left[\alpha_{0}, \alpha_{1}\right] \rightarrow D$ which admits adiabatic solutions such that all eigenvalues of $D_{2} f(\alpha, s(\alpha))$ have negative real part for $\alpha \in\left[\alpha_{0}, \alpha_{1}\right]$. Moreover, let $\mu:\left[\alpha_{0}, \alpha_{1}\right] \times(0, \tilde{\varepsilon}) \rightarrow \mathbb{R}^{N}$ be a function describing corresponding adiabatic solutions. Then, there exists an $\hat{\varepsilon}>0$ such that for all $\varepsilon \in(0, \hat{\varepsilon})$, the solution $\mu(\cdot, \varepsilon)$ is $\left(\alpha_{0}, \alpha_{1}-\alpha_{0}\right)$-attractive.

PROOF. Lemma 5.4.3 implies the existence of $\delta>0, K \geq 1$ and $\gamma<0$ with the properties mentioned in the lemma. We choose $\varepsilon^{*}>0$ such that

$$
\sup _{\alpha \in\left[\alpha_{0}, \alpha_{1}\right]}\|\mu(\alpha, \varepsilon)-s(\alpha)\|<\delta \quad \text { for all } \varepsilon \in\left(0, \varepsilon^{*}\right)
$$

and

$$
K \exp \left(\frac{\gamma}{\hat{\varepsilon}}\left(\alpha_{1}-\alpha_{0}\right)\right)<1
$$

By applying Lemma 5.4.3, we obtain that the transition operator $\Lambda_{\varepsilon}$ of the variational equation

$$
\dot{x}=\frac{1}{\varepsilon} D_{2} f(t, \mu(t, \varepsilon)) x
$$

satisfies $\left\|\Lambda_{\varepsilon}\left(\alpha_{1}, \alpha_{0}\right)\right\|<1$. Hence, Theorem 5.3.2 implies that there exists an $\hat{\varepsilon} \in\left(0, \varepsilon^{*}\right)$ such that for all $\varepsilon \in(0, \hat{\varepsilon})$, the solution $\mu(\cdot, \varepsilon)$ is $\left(\alpha_{0}, \alpha_{1}-\alpha_{0}\right)$-attractive (due to Remark 5.3.3 (ii), the condition on the nonlinearity is fulfilled for small $\varepsilon$ ).

For the main result of this section, recall the Hypothesis from the beginning of this section.

5.4.5 Theorem (Relationship to The Concept of Finite-Time Bifurcation). We assume that the equilibrium branch $s_{1}$ admits adiabatic solutions, i.e., there exists a function $\mu:\left[\alpha_{-}, \alpha_{+}\right] \times(0, \tilde{\varepsilon}) \rightarrow \mathbb{R}^{N}$ such that $\mu(\cdot, \varepsilon)$ is a solution of $(5.25)_{\varepsilon}$ and we have

$$
\lim _{\varepsilon \searrow 0} \sup _{\alpha \in\left[\alpha_{-}, \alpha_{+}\right]}\left\|\mu(\alpha, \varepsilon)-s_{1}(\alpha)\right\|=0 .
$$

Then, for sufficiently small $\alpha>\alpha_{-}$and $\varepsilon>0$, the solution $\mu(\cdot, \varepsilon)$ is $\left(\alpha, \alpha_{+}-\alpha\right)$-attractive, and the limit relation

$$
\lim _{\alpha \searrow \alpha_{-}} \limsup _{\varepsilon \unlhd 0} \mathfrak{A}_{\mu(\cdot, \varepsilon)}^{\left(\alpha, \alpha_{+}-\alpha\right)}=0
$$

is satisfied.

Proof. Since $\lim _{\alpha \rightarrow \alpha_{-}} s_{1}(\alpha)=\lim _{\alpha \rightarrow \alpha_{-}} s_{2}(\alpha)$, there exists an $\hat{\alpha} \in\left(\alpha_{-}, \alpha_{+}\right)$with

$$
\left\|s_{1}(\alpha)-s_{2}(\alpha)\right\|<\frac{1}{3}\left\|s_{1}\left(\alpha_{+}\right)-s_{2}\left(\alpha_{+}\right)\right\| \text {for all } \alpha \in\left[\alpha_{-}, \hat{\alpha}\right] \text {. }
$$

Now, we prove the following statement which is obviously sufficient for the assertion: For all $\alpha \in\left(\alpha_{-}, \hat{\alpha}\right]$, there exists an $\hat{\varepsilon}>0$ such that for all $\varepsilon \in(0, \hat{\varepsilon})$, the solution $\mu(\cdot, \varepsilon)$ is $\left(\alpha, \alpha_{+}-\alpha\right)$ attractive and

$$
\mathfrak{A}_{\mu(\cdot, \varepsilon)}^{\left(\alpha, \alpha_{+}-\alpha\right)} \leq \frac{3}{2}\left\|s_{1}(\alpha)-s_{2}(\alpha)\right\|
$$

is fulfilled. We choose $\alpha \in\left(\alpha_{-}, \hat{\alpha}\right]$ arbitrarily. It follows from Theorem 5.4.2 that there exists a function $v:\left[\alpha, \alpha_{+}\right] \times(0, \tilde{\varepsilon}) \rightarrow \mathbb{R}^{N}$ such that $v(\cdot, \varepsilon)$ is a solution of $(5.25)_{\varepsilon}$ and

$$
\lim _{\varepsilon \searrow 0} \sup _{\tilde{\alpha} \in\left[\alpha, \alpha_{+}\right]}\left\|v(\tilde{\alpha}, \varepsilon)-s_{2}(\tilde{\alpha})\right\|=0 .
$$


Thus, there exists an $\hat{\varepsilon}>0$ such that for all $\varepsilon \in(0, \hat{\varepsilon})$, we have

$$
\begin{gathered}
\sup _{\tilde{\alpha} \in\left[\alpha, \alpha_{+}\right]}\left\|v(\tilde{\alpha}, \varepsilon)-s_{2}(\tilde{\alpha})\right\|<\frac{1}{4}\left\|s_{1}(\alpha)-s_{2}(\alpha)\right\|, \\
\sup _{\tilde{\alpha} \in\left[\alpha, \alpha_{+}\right]}\left\|\mu(\tilde{\alpha}, \varepsilon)-s_{1}(\tilde{\alpha})\right\|<\frac{1}{4}\left\|s_{1}(\alpha)-s_{2}(\alpha)\right\|
\end{gathered}
$$

and $\mu(\cdot, \varepsilon)$ is $\left(\alpha, \alpha_{+}-\alpha\right)$-attractive (cf. Corollary 5.4.4). For all $\varepsilon \in(0, \hat{\varepsilon})$, this implies the relations

$$
\begin{aligned}
\|v(\alpha, \varepsilon)-\mu(\alpha, \varepsilon)\| & =\left\|v(\alpha, \varepsilon)-s_{2}(\alpha)+s_{2}(\alpha)-s_{1}(\alpha)+s_{1}(\alpha)-\mu(\alpha, \varepsilon)\right\| \\
& \leq\left\|v(\alpha, \varepsilon)-s_{2}(\alpha)\right\|+\left\|s_{2}(\alpha)-s_{1}(\alpha)\right\|+\left\|s_{1}(\alpha)-\mu(\alpha, \varepsilon)\right\| \\
& <\frac{3}{2}\left\|s_{1}(\alpha)-s_{2}(\alpha)\right\| .
\end{aligned}
$$

and

$$
\|\underbrace{\lambda_{\varepsilon}\left(\alpha_{+}, \alpha, \nu(\alpha, \varepsilon)\right)}_{\nu\left(\alpha_{+}, \varepsilon\right)}-\mu\left(\alpha_{+}, \varepsilon\right)\|>\frac{1}{2}\left\|s_{1}\left(\alpha_{+}\right)-s_{2}\left(\alpha_{+}\right)\right\| \stackrel{(5.29)}{>} \frac{3}{2}\left\|s_{1}(\alpha)-s_{2}(\alpha)\right\|,
$$

where $\lambda_{\varepsilon}$ denotes the general solution of $(5.25)_{\varepsilon}$. This finishes the proof of this theorem. 


\section{CHAPTER 6}

\section{BIFURCATIONS IN DimENSION ONE}

The aim of this chapter is to develop nonautonomous counterparts for the classical onedimensional bifurcation patterns such as the transcritical and pitchfork bifurcation, both for nonautonomous bifurcations and transitions.

In this chapter, only the continuous case of ordinary differential equations is treated. For analogous results in the context of difference equations, see RASMUSSEN [144].

Recently, LANGA \& ROBINSON \& SUÁREZ [104] also studied the occurrence of one-dimensional nonautonomous bifurcations, which they understand as merging processes of two distinct solutions with different stability behavior. As in this chapter, their theorems are formulated in terms of Taylor coefficients for the right hand side of an ordinary differential equation. These conditions, however, are of a quite different form than the results obtained in this chapter. This difference is due to fact that, in [104], explicitly solvable models are used to formulate these conditions.

Stochastic versions (in the sense of a D-bifurcation, cf. Subsection 2.4.3) of the transcritical and pitchfork bifurcation are examined in the thesis of STEINKAMP [176] (see also CRAUEL \& IMKELLER \& STEINKAMP [58]).

\subsection{NONAUTONOMOUS TRANSCRITICAL BIFURCATION}

In this section, nonautonomous generalizations of the classical transcritical bifurcation are derived. First, the case of unbounded time domains is treated.

6.1.1 Theorem (Nonautonomous Transcritical Bifurcation, Part I). Let $x_{-}<0<x_{+}$and $\alpha_{-}<\alpha_{+}$be in $\overline{\mathbb{R}}$ and $\square$ be an unbounded interval of the form $\mathbb{R}_{\kappa}^{-}, \mathbb{R}_{\kappa}^{+}$ or $\mathbb{R}$, respectively, and consider the nonautonomous differential equation

$$
\dot{x}=a(t, \alpha) x+b(t, \alpha) x^{2}+r(t, x, \alpha)
$$

with continuous functions $a: \llbracket \times\left(\alpha_{-}, \alpha_{+}\right) \rightarrow \mathbb{R}, b: \rrbracket \times\left(\alpha_{-}, \alpha_{+}\right) \rightarrow \mathbb{R}$ and $r: \llbracket \times\left(x_{-}, x_{+}\right) \times$

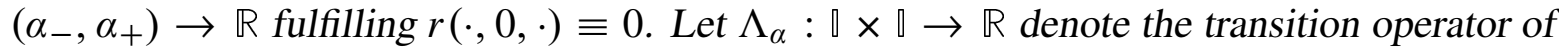
the linearized equation $\dot{x}=a(t, \alpha) x$, and assume, there exists an $\alpha_{0} \in\left(\alpha_{-}, \alpha_{+}\right)$such that the following hypotheses hold:

- Hypothesis on linear PaRT. There exist two functions $\beta_{1}, \beta_{2}:\left(\alpha_{-}, \alpha_{+}\right) \rightarrow \mathbb{R}$ which are either both monotone increasing or both monotone decreasing and $K \geq 1$ such that $\lim _{\alpha \rightarrow \alpha_{0}} \beta_{1}(\alpha)=\lim _{\alpha \rightarrow \alpha_{0}} \beta_{2}(\alpha)=0$ and 


$$
\begin{array}{ll}
\Lambda_{\alpha}(t, s) \leq K e^{\beta_{1}(\alpha)(t-s)} & \text { for all } \alpha \in\left(\alpha_{-}, \alpha_{+}\right) \text {and } t, s \in \llbracket \text { with } t \geq s, \\
\Lambda_{\alpha}(t, s) \leq K e^{\beta_{2}(\alpha)(t-s)} & \text { for all } \alpha \in\left(\alpha_{-}, \alpha_{+}\right) \text {and } t, s \in \llbracket \text { with } t \leq s .
\end{array}
$$

- HyPOTHESIS ON NONLINEARITY. The quadratic term either fulfills

$$
0<\liminf _{\alpha \rightarrow \alpha_{0}} \inf _{t \in \mathbb{\complement}} b(t, \alpha) \leq \limsup _{\alpha \rightarrow \alpha_{0}} \sup _{t \in \mathbb{\prod}} b(t, \alpha)<\infty
$$

or

$$
-\infty<\liminf _{\alpha \rightarrow \alpha_{0}} \inf _{t \in \rrbracket} b(t, \alpha) \leq \limsup _{\alpha \rightarrow \alpha_{0}} \sup _{t \in \rrbracket} b(t, \alpha)<0,
$$

and the remainder satisfies

$$
\lim _{x \rightarrow 0} \sup _{\alpha \in\left(\alpha_{0}-|x|, \alpha_{0}+|x|\right)} \sup _{t \in \mathbb{\prod}} \frac{|r(t, x, \alpha)|}{|x|^{2}}=0
$$

and

$$
\limsup _{\alpha \rightarrow \alpha_{0}} \limsup _{x \rightarrow 0} \sup _{t \in \rrbracket} \frac{2 K|r(t, x, \alpha)|}{|x| \max \left\{-\beta_{1}(\alpha), \beta_{2}(\alpha)\right\}}<1 \text {. }
$$

Then, there exist $\hat{\alpha}_{-}<0<\hat{\alpha}_{+}$such that the following statements are fulfilled:

(i) In case the functions $\beta_{1}$ and $\beta_{2}$ are monotone increasing, the trivial solution is past (future, all-time, respectively) attractive for $\alpha \in\left(\hat{\alpha}_{-}, \alpha_{0}\right)$ and past (future, all-time, respectively) repulsive for $\alpha \in\left(\alpha_{0}, \hat{\alpha}_{+}\right)$. The differential equation (6.1 $)_{\alpha}$ admits a past (future, all-time, respectively) bifurcation, since the corresponding radii of past (future, all-time, respectively) attraction and repulsion satisfy

$$
\lim _{\alpha>\alpha_{0}} \mathfrak{A}_{0}^{\alpha}=0 \quad \text { and } \quad \lim _{\alpha \triangleleft \alpha_{0}} \mathfrak{R}_{0}^{\alpha}=0 .
$$

(ii) In case the functions $\beta_{1}$ and $\beta_{2}$ are monotone decreasing, the trivial solution is past (future, all-time, respectively) repulsive for $\alpha \in\left(\hat{\alpha}_{-}, \alpha_{0}\right)$ and past (future, all-time, respectively) attractive for $\alpha \in\left(\alpha_{0}, \hat{\alpha}_{+}\right)$. The differential equation $(6.1)_{\alpha}$ admits a past (future, all-time, respectively) bifurcation, since the corresponding radii of past (future, all-time, respectively) repulsion and attraction satisfy

$$
\lim _{\alpha>\alpha_{0}} \mathfrak{R}_{0}^{\alpha}=0 \quad \text { and } \quad \lim _{\alpha \searrow \alpha_{0}} \mathfrak{A}_{0}^{\alpha}=0 .
$$

PROOF. First of all, we assume w.l.o.g. that $K>1$. Let $\lambda_{\alpha}$ denote the general solution of $(6.1)_{\alpha}$. We will only prove assertion (i), since the proof of (ii) is similar. The functions $\beta_{1}$ and $\beta_{2}$ are therefore monotone increasing. W.1.o.g., we only treat the case (6.2). We choose $\hat{\alpha}_{-}<\alpha_{0}<\hat{\alpha}_{+}$ such that

$$
0<\inf _{\alpha \in\left(\hat{\alpha}_{-}, \hat{\alpha}_{+}\right), t \in \llbracket} b(t, \alpha) \leq \sup _{\alpha \in\left(\hat{\alpha}_{-}, \hat{\alpha}_{+}\right), t \in \mathbb{}} b(t, \alpha)<\infty
$$

(cf. (6.2)) and

$$
\limsup _{x \rightarrow 0} \sup _{t \in \llbracket} \frac{|r(t, x, \alpha)|}{|x|}<\frac{-\min \left\{\beta_{1}(\alpha),-\beta_{2}(\alpha)\right\}}{2 K} \text { for all } \alpha \in\left(\hat{\alpha}_{-}, \hat{\alpha}_{+}\right) .
$$

(cf. (6.5)). Because of these two relations, Theorem 5.3.1 can be applied, and the attractivity and 
repulsivity of the trivial solutions as stated in the theorem follows. Assume to the contrary that

$$
\eta:=\limsup _{\alpha>\alpha_{0}} \mathfrak{A}_{0}^{\alpha}>0
$$

holds. Due to (6.6) and (6.4), there exist $\tilde{\alpha}_{-} \in\left(\hat{\alpha}_{-}, \alpha_{0}\right), \xi \in(0, \eta)$ and $L \in\left(0, \frac{\xi}{4 K}\right)$ with

$$
b(t, \alpha) x^{2}+r(t, x, \alpha)>L \quad \text { for all } t \in \mathbb{\mathbb { N }}, \alpha \in\left(\tilde{\alpha}_{-}, \alpha_{0}\right) \text { and } x \in\left[\frac{\xi}{2 K^{2}}, \xi\right] .
$$

We fix $\hat{\alpha} \in\left(\tilde{\alpha}_{-}, \alpha_{0}\right)$ such that $\mathfrak{A}_{0}^{\hat{\alpha}}>\xi$ and $\beta_{2}(\hat{\alpha}) \geq \beta:=-\frac{2 K L}{\xi}>-\frac{1}{2}$. For arbitrary $\tau \in \mathbb{\mathbb { V }}$, the solution $\mu_{\tau}(\cdot):=\lambda_{\hat{\alpha}}(\cdot, \tau, \xi)$ of $(6.1) \hat{\alpha}$ is also a solution of the inhomogeneous linear differential equation

$$
\dot{x}=a(t, \hat{\alpha}) x+b(t, \hat{\alpha})\left(\mu_{\tau}(t)\right)^{2}+r\left(t, \mu_{\tau}(t), \hat{\alpha}\right) .
$$

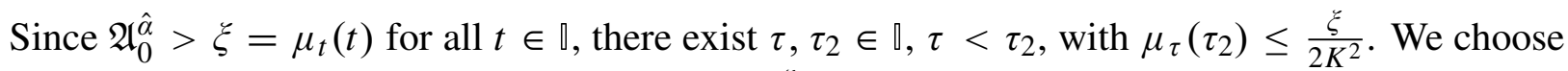
$\tau_{2}$ minimal with this property, i.e., $\mu_{\tau}(t) \geq \frac{\xi}{2 K^{2}}$ for all $t \in\left[\tau, \tau_{2}\right]$. Furthermore, we choose $\tau_{1} \in\left[\tau, \tau_{2}\right]$ such that

$$
\mu_{\tau}\left(\tau_{1}\right)=\frac{\xi}{2 K} \quad \text { and } \quad \mu_{\tau}(t) \in\left[\frac{\xi}{2 K^{2}}, \xi\right] \quad \text { for all } t \in\left[\tau_{1}, \tau_{2}\right] .
$$

Therefore, and due to (6.7) and the variation of constants formula (cf. Proposition A.1.6), applied to $(6.8)$, the relation

$$
\begin{aligned}
\mu_{\tau}\left(\tau_{2}\right) & =\Lambda_{\hat{\alpha}}\left(\tau_{2}, \tau_{1}\right) \mu_{\tau}\left(\tau_{1}\right)+\int_{\tau_{1}}^{\tau_{2}} \Lambda_{\hat{\alpha}}\left(\tau_{2}, t\right)\left(b(t, \hat{\alpha})\left(\mu_{\tau}(t)\right)^{2}+r\left(t, \mu_{\tau}(t), \hat{\alpha}\right)\right) d t \\
& >\frac{\xi}{2 K^{2}} e^{\beta\left(\tau_{2}-\tau_{1}\right)}+\frac{L}{K} \int_{\tau_{1}}^{\tau_{2}} e^{\beta\left(\tau_{2}-t\right)} d t \\
& =e^{\beta\left(\tau_{2}-\tau_{1}\right)} \underbrace{\left(\frac{\xi}{2 K^{2}}+\frac{L}{K \beta}\right)}_{=0}-\frac{L}{K \beta}=\frac{\xi}{2 K^{2}}
\end{aligned}
$$

holds $\left(K>1\right.$ implies $\tau_{1}<\tau_{2}$ ). This is a contradiction and proves $\lim _{\alpha>\alpha_{0}} \mathfrak{A}_{0}^{\alpha}=0$. Analogously, one can show $\lim _{\alpha \triangleleft \alpha_{0}} \mathfrak{R}_{0}^{\alpha}=0$ and treat the case (6.3).

\subsubsection{REMARKS.}

(i) In the limit $\alpha \rightarrow \alpha_{0}$, the attractivity or repulsivity of the trivial solution is only lost in one direction, i.e., nonautonomous transcritical bifurcations are partial bifurcations. For instance, in case the functions $\beta_{1}, \beta_{2}$ are monotone increasing and (6.2) is satisfied, there exists a $\gamma<0$ such that $(\gamma, 0]$ is attracted by the trivial solution of $(6.4)_{\alpha}$ for $\alpha \in\left(\hat{\alpha}_{-}, \alpha_{0}\right)$ in the sense of past, future or all-time attractivity, respectively.

(ii) The Hypothesis on the linear part implies that the past (future, all-time, respectively) dichotomy spectrum of the linearization $\dot{x}=a(t, \alpha) x$ converges to $\{0\}$ in Hausdorff distance in the limit $\alpha \rightarrow \alpha_{0}$.

(iii) Condition (6.5) is only used to obtain the attractivity or repulsivity of the trivial solution by applying Theorem 5.3.1. Alternatively, one can directly postulate that the trivial solution changes their stability at the parameter value $\alpha_{0}$ from, say, attractivity to repulsivity. 
(iv) Please note that the above bifurcation result is essentially the combination of two scenarios which are independent of each other. This means that it is possible to consider $(6.1)_{\alpha}$ only for $\alpha>\alpha_{0}$ or $\alpha<\alpha_{0}$, respectively, in order to obtain the results which apply for these parameter values.

The following example shows that Theorem 6.1.1 is indeed a nonautonomous generalization of the well-known autonomous result.

6.1.3 EXAmple. Let $x_{-}<0<x_{+}$and $\alpha_{-}<0<\alpha_{+}$be in $\overline{\mathbb{R}}$, and consider the autonomous differential equation

$$
\dot{x}=f(x, \alpha),
$$

where the $C^{4}$-function $f:\left(x_{-}, x_{+}\right) \times\left(\alpha_{-}, \alpha_{+}\right) \rightarrow \mathbb{R}$ satisfies the following assumptions:

(i) $f(0, \alpha)=0$ for all $\alpha \in\left(\alpha_{-}, \alpha_{+}\right)$,

(ii) $D_{1} f(0,0)=0$,

(iii) $D_{1} D_{2} f(0,0) \neq 0$,

(iv) $D_{1}^{2} f(0,0) \neq 0$.

Please note that (i) implies $D_{2}^{n} f(0, \alpha)=0$ for all $\alpha \in\left(\alpha_{-}, \alpha_{+}\right)$and $n \in \mathbb{N}$. Then, (6.9) admits an autonomous transcritical bifurcation (see, e.g., WigGINS [181, p. 265 f.] and AULBACH [14, Satz 7.10.6]), i.e., there exist a neighborhood $U \times V$ of $(0,0)$ in $\mathbb{R}^{2}$ and a $C^{1}$-function $h: U \rightarrow V$ with $h(0)=0$ and

$$
f(x, h(x))=0 \text { for all } x \in U .
$$

Except the trivial equilibria and the equilibria described by $h$, there are no other equilibria in $U \times V$. Now, we will show that this example fulfills the hypotheses of Theorem 6.1.1. Thereto, we write the second order Taylor expansion of $f$ (see, e.g., LANG [101, p. 349]):

$$
f(x, \alpha)=\underbrace{D_{1} D_{2} f(0,0) \alpha}_{=: \bar{a}(\alpha)} x+\underbrace{\frac{1}{2} D_{1}^{2} f(0,0)}_{=: \bar{b}(\alpha)} x^{2}+r(x, \alpha)
$$

where

$$
\begin{array}{r}
r(x, \alpha)=\int_{0}^{1} \frac{(1-t)^{2}}{2}\left(D_{1}^{3} f(t x, t \alpha) x^{3}+3 D_{1}^{2} D_{2} f(t x, t \alpha) x^{2} \alpha+\right. \\
\left.3 D_{1} D_{2}^{2} f(t x, t \alpha) x \alpha^{2}+D_{2}^{3} f(t x, t \alpha) \alpha^{3}\right) d t
\end{array}
$$

Obviously, the Hypothesis on the linear part are fulfilled (with $\beta_{1}(\alpha):=\beta_{2}(\alpha):=\bar{a}(\alpha)$ and $K:=1)$, and (6.2) or (6.3) holds, since the above defined function $\bar{b}$ is constant. Furthermore, the representation for the remainder implies that

$$
\lim _{x \rightarrow 0} \sup _{\alpha \in(-|x|,|x|)} \frac{|r(x, \alpha)|}{|x|^{2}}=0
$$

and

$$
\limsup _{x \rightarrow 0} \frac{|r(x, \alpha)|}{|x|} \leq \alpha^{2} \int_{0}^{1} \frac{(1-t)^{2}}{2}\left(\left|3 D_{1} D_{2}^{2} f(0, t \alpha)\right|+t\left|D_{1} D_{2}^{3} f(0, t \alpha) \alpha\right|\right) d t .
$$

This means that (6.5) holds, since $\max \left\{-\beta_{1}(\alpha), \beta_{2}(\alpha)\right\}$ depends linearly in $\alpha$. Therefore, all hypotheses of Theorem 6.1.1 are fulfilled, and thus, this example shows that Theorem 6.1.1 is a proper generalization of the well-known autonomous transcritical bifurcation pattern. 
In case of compact time domains, the following result is obtained.

6.1.4 Theorem (Nonautonomous Transcritical Bifurcation, Part II). Let

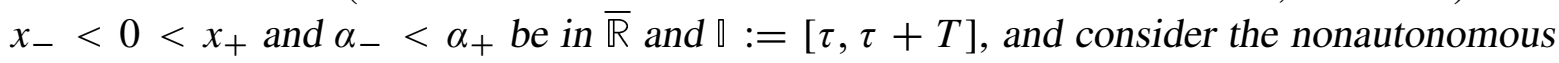
differential equation

$$
\dot{x}=a(t, \alpha) x+b(t, \alpha) x^{2}+r(t, x, \alpha)
$$

with continuous functions $a: \llbracket \times\left(\alpha_{-}, \alpha_{+}\right) \rightarrow \mathbb{R}, b: \llbracket \times\left(\alpha_{-}, \alpha_{+}\right) \rightarrow \mathbb{R}$ and $r: \llbracket \times\left(x_{-}, x_{+}\right) \times$

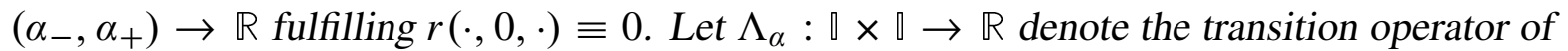
the linearized equation $\dot{x}=a(t, \alpha) x$. We define

$$
K(\alpha):=\sup \left\{\Lambda_{\alpha}(t, s): t, s \in \mathbb{}\left(\text { for all } \alpha \in\left(\alpha_{-}, \alpha_{+}\right)\right.\right.
$$

and assume, there exists an $\alpha_{0} \in\left(\alpha_{-}, \alpha_{+}\right)$such that the following hypotheses hold:

- HYPOTHESIS ON LINEAR PART. We either have

$$
\begin{array}{ll}
\Lambda_{\alpha}(\tau+T, \tau)<1 & \text { for all } \alpha \in\left(\alpha_{-}, \alpha_{0}\right) \text { and } \\
\Lambda_{\alpha}(\tau+T, \tau)>1 & \text { for all } \alpha \in\left(\alpha_{0}, \alpha_{+}\right)
\end{array}
$$

or

$$
\begin{array}{ll}
\Lambda_{\alpha}(\tau+T, \tau)>1 & \text { for all } \alpha \in\left(\alpha_{-}, \alpha_{0}\right) \text { and } \\
\Lambda_{\alpha}(\tau+T, \tau)<1 & \text { for all } \alpha \in\left(\alpha_{0}, \alpha_{+}\right) .
\end{array}
$$

- HyPOTHESIS ON NONLINEARITY. The quadratic term either fulfills

$$
\liminf _{\alpha \rightarrow \alpha_{0}} \inf _{t \in \llbracket} b(t, \alpha)>0
$$

or

$$
\limsup _{\alpha \rightarrow \alpha_{0}} \sup _{t \in \llbracket} b(t, \alpha)<0,
$$

and the remainder satisfies

$$
\lim _{x \rightarrow 0} \sup _{\alpha \in\left(\alpha_{0}-|x|, \alpha_{0}+|x|\right)} \sup _{t \in \rrbracket} \frac{|r(t, x, \alpha)|}{|x|^{2}}=0
$$

and

$$
\limsup _{\alpha \rightarrow \alpha_{0}} \limsup _{x \rightarrow 0} \sup _{t \in \rrbracket}-\frac{T K(\alpha)|r(t, x, \alpha)|}{|x| \ln \left(\min \left\{\Lambda_{\alpha}(\tau+T, \tau), \Lambda_{\alpha}(\tau, \tau+T)\right\}\right)}<1 .
$$

Then, there exist $\hat{\alpha}_{-}<0<\hat{\alpha}_{+}$such that the following statements are fulfilled:

(i) In case (6.11), the trivial solution is $(\tau, T)$-attractive for $\alpha \in\left(\hat{\alpha}_{-}, \alpha_{0}\right)$ and $(\tau, T)$ repulsive for $\alpha \in\left(\alpha_{0}, \hat{\alpha}_{+}\right)$. The differential equation $(6.10)_{\alpha}$ admits a $(\tau, T)$ bifurcation, since the corresponding radii of $(\tau, T)$-attraction and repulsion satisfy

$$
\lim _{\alpha>\alpha_{0}} \mathfrak{A}_{0}^{\alpha}=0 \quad \text { and } \quad \lim _{\alpha \searrow \alpha_{0}} \mathfrak{R}_{0}^{\alpha}=0
$$

(ii) In case (6.11), the trivial solution is $(\tau, T)$-repulsive for $\alpha \in\left(\hat{\alpha}_{-}, \alpha_{0}\right)$ and $(\tau, T)$ attractive for $\alpha \in\left(\alpha_{0}, \hat{\alpha}_{+}\right)$. The differential equation $(6.10)_{\alpha}$ admits a $(\tau, T)$ bifurcation, since the corresponding radii of $(\tau, T)$-repulsion and attraction satisfy 


$$
\lim _{\alpha>\alpha_{0}} \mathfrak{R}_{0}^{\alpha}=0 \quad \text { and } \quad \lim _{\alpha \searrow \alpha_{0}} \mathfrak{A}_{0}^{\alpha}=0
$$

PROOF. Let $\lambda_{\alpha}$ denote the general solution of $(6.10)_{\alpha}$. We will only prove assertion (i), since the proof of (ii) is similar. Therefore, (6.11) is fulfilled. W.l.o.g., we only treat the case (6.13). We choose $\hat{\alpha}_{-}<0<\hat{\alpha}_{+}$such that

$$
\inf _{\alpha \in\left(\hat{\alpha}_{-}, \hat{\alpha}_{+}\right), t \in \llbracket} b(t, \alpha)>0
$$

and

$$
\limsup _{x \rightarrow 0} \sup _{t \in \mathbb{\rrbracket}} \frac{|r(t, x, \alpha)|}{|x|} \leq-\gamma \frac{\ln \left(\min \left\{\Lambda_{\alpha}(\tau+T, \tau), \Lambda_{\alpha}(\tau, \tau+T)\right\}\right)}{T K(\alpha)} \quad \text { for all } \alpha \in\left(\hat{\alpha}_{-}, \hat{\alpha}_{+}\right)
$$

for some $\gamma \in(0,1)$. Because of these two relations, Theorem 5.3.2 can be applied, and the attractivity and repulsivity of the trivial solutions as stated in the theorem follows. We define

$$
K_{-}:=\inf \left\{\Lambda_{\alpha}(t, s): t, s \in \mathbb{\mathbb { N }}, \alpha \in\left[\hat{\alpha}_{-}, \alpha_{0}\right]\right\} \in(0,1) .
$$

Assume to the contrary that

$$
\eta:=\limsup _{\alpha>\alpha_{0}} \mathfrak{A}_{0}^{\alpha}>0
$$

holds. Due to (6.17) and (6.15), there exist $\tilde{\alpha}_{-} \in\left(\hat{\alpha}_{-}, \alpha_{0}\right), \xi \in\left(0, K_{-} \eta\right)$ and $L>0$ with

$$
b(t, \alpha) x^{2}+r(t, x, \alpha)>L \quad \text { for all } t \in \mathbb{1}, \alpha \in\left(\tilde{\alpha}_{-}, \alpha_{0}\right) \text { and } x \in\left[K_{-} \xi, \frac{\xi}{K_{-}}\right] .
$$

We fix $\hat{\alpha} \in\left(\tilde{\alpha}_{-}, \alpha_{0}\right)$ such that $\mathfrak{A}_{0}^{\hat{\alpha}}>\xi$ and

$$
\Lambda_{\hat{\alpha}}(\tau+T, \tau) \geq 1-\frac{K_{-} L T}{\xi}
$$

For arbitrary $\tau \in \mathbb{Q}$, the solution $\mu_{\tau}(\cdot):=\lambda_{\hat{\alpha}}(\cdot, \tau, \xi)$ of $(6.10)_{\hat{\alpha}}$ is also a solution of the inhomogeneous linear differential equation

$$
\dot{x}=a(t, \hat{\alpha}) x+b(t, \hat{\alpha})\left(\mu_{\tau}(t)\right)^{2}+r\left(t, \mu_{\tau}(t), \hat{\alpha}\right) .
$$

Since $\mathfrak{A}_{0}^{\hat{\alpha}}>\xi$, we have

$$
\mu_{\tau}(\tau+T)<\xi .
$$

Moreover, from the definition of $K_{-}$and (6.18), we directly get

$$
\mu_{\tau}(\tau+t) \geq K_{-} \xi \text { for all } t \in[0, T]
$$

We distinguish two cases.

CASE 1 . There exists a $\bar{t} \in(0, T]$ such that

$$
\mu_{\tau}(\tau+\bar{t})=\frac{\xi}{K_{-}} .
$$


We choose $\bar{t}$ maximal with this property. Due to (6.21), this means that $\mu(\tau+t) \leq \frac{\xi}{K_{-}}$for all $t \in[\bar{t}, T]$. Then, the variation of constants formula (cf. Proposition A.1.6), applied to (6.20), implies the relation

$$
\begin{aligned}
& \mu_{\tau}(\tau+T) \\
= & \Lambda_{\hat{\alpha}}(\tau+T, \tau+\bar{t}) \frac{\xi}{K_{-}}+\int_{\tau+\bar{t}}^{\tau+T} \Lambda_{\hat{\alpha}}(\tau+T, t)\left(b(t, \hat{\alpha})\left(\mu_{\tau}(t)\right)^{2}+r\left(t, \mu_{\tau}(t), \hat{\alpha}\right)\right) d t \\
\stackrel{(6.18)}{\geq} \xi+K_{-} L(T-\bar{t})>\xi . &
\end{aligned}
$$

This contradicts (6.21).

CASE 2. For all $t \in(0, T]$, we have

$$
\mu_{\tau}(\tau+\bar{t})<\frac{\xi}{K_{-}}
$$

In this case, the variation of constants formula, applied to (6.20), yields

$$
\begin{aligned}
& \mu_{\tau}(\tau+T) \\
&= \Lambda_{\hat{\alpha}}(\tau+T, \tau) \xi+\int_{\tau}^{\tau+T} \Lambda_{\hat{\alpha}}(\tau+T, t)\left(b(t, \hat{\alpha})\left(\mu_{\tau}(t)\right)^{2}+r\left(t, \mu_{\tau}(t), \hat{\alpha}\right)\right) d t \\
& \stackrel{(6.18),(6.19)}{\geq}\left(1-\frac{K_{-} L T}{\xi}\right) \xi+K_{-} L T=\xi .
\end{aligned}
$$

This contradicts (6.21) also, and thus, $\lim _{\alpha>\alpha_{0}} \mathfrak{A}_{0}^{\alpha}=0$ is proved. Analogously, one can show $\lim _{\alpha \triangleleft \alpha_{0}} \mathfrak{R}_{0}^{\alpha}=0$ and treat the case (6.14).

\subsubsection{REMARKS.}

(i) The Hypothesis on the linear part implies that the $(\tau, T)$-dichotomy spectrum of the linearization $\dot{x}=a(t, \alpha) x$ converges to $\{0\}$ in Hausdorff distance in the limit $\alpha \rightarrow \alpha_{0}$.

(ii) Condition (6.16) is only used to obtain the attractivity or repulsivity of the trivial solution by applying Theorem 5.3.2. Alternatively, one can directly postulate that the trivial solution changes their stability at the parameter value $\alpha_{0}$ from, say, attractivity to repulsivity.

\subsection{Nonautonomous PitchFork Bifurcation}

In this section, nonautonomous generalizations of the classical pitchfork bifurcation are derived. First, the case of unbounded time domains is treated.

6.2.1 Theorem (Nonautonomous Pitchfork Bifurcation, Part I). Let $x_{-}<$

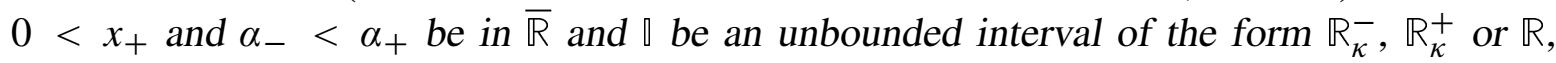
respectively, and consider the nonautonomous differential equation

$$
\dot{x}=a(t, \alpha) x+b(t, \alpha) x^{3}+r(t, x, \alpha)
$$

with continuous functions $a: \llbracket \times\left(\alpha_{-}, \alpha_{+}\right) \rightarrow \mathbb{R}, b: \llbracket \times\left(\alpha_{-}, \alpha_{+}\right) \rightarrow \mathbb{R}$ and $r: \llbracket \times\left(x_{-}, x_{+}\right) \times$ $\left(\alpha_{-}, \alpha_{+}\right) \rightarrow \mathbb{R}$ fulfilling $r(\cdot, 0, \cdot) \equiv 0$. Let $\Lambda_{\alpha}: \mathbb{x} \times \mathbb{\mathbb { R }}$ be the transition operator of the linearized equation $\dot{x}=a(t, \alpha) x$, and assume, there exists an $\alpha_{0} \in\left(\alpha_{-}, \alpha_{+}\right)$such that the following hypotheses hold: 
- Hypothesis on linear PaRT. There exist two functions $\beta_{1}, \beta_{2}:\left(\alpha_{-}, \alpha_{+}\right) \rightarrow \mathbb{R}$ which are either both monotone increasing or both monotone decreasing and $K \geq 1$ such that $\lim _{\alpha \rightarrow \alpha_{0}} \beta_{1}(\alpha)=\lim _{\alpha \rightarrow \alpha_{0}} \beta_{2}(\alpha)=0$ and

$$
\begin{aligned}
& \Lambda_{\alpha}(t, s) \leq K e^{\beta_{1}(\alpha)(t-s)} \quad \text { for all } \alpha \in\left(\alpha_{-}, \alpha_{+}\right) \text {and } t, s \in \mathbb{\square} \text { with } t \geq s \text {, } \\
& \Lambda_{\alpha}(t, s) \leq K e^{\beta_{2}(\alpha)(t-s)} \quad \text { for all } \alpha \in\left(\alpha_{-}, \alpha_{+}\right) \text {and } t, s \in \mathbb{\square} \text { with } t \leq s \text {. }
\end{aligned}
$$

- HyPOTHESIS ON NONLINEARITY. The cubic term either fulfills

$$
0<\liminf _{\alpha \rightarrow \alpha_{0}} \inf _{t \in \mathbb{\complement}} b(t, \alpha) \leq \limsup _{\alpha \rightarrow \alpha_{0}} \sup _{t \in \rrbracket} b(t, \alpha)<\infty
$$

or

$$
-\infty<\liminf _{\alpha \rightarrow \alpha_{0}} \inf _{t \in \rrbracket} b(t, \alpha) \leq \limsup _{\alpha \rightarrow \alpha_{0}} \sup _{t \in \llbracket} b(t, \alpha)<0,
$$

and the remainder satisfies

$$
\lim _{x \rightarrow 0} \sup _{\alpha \in\left(\alpha_{0}-x^{2}, \alpha_{0}+x^{2}\right)} \sup _{t \in \mathbb{\complement}} \frac{|r(t, x, \alpha)|}{|x|^{3}}=0
$$

and

$$
\limsup _{\alpha \rightarrow \alpha_{0}} \limsup _{x \rightarrow 0} \sup _{t \in \mathbb{\Xi}} \frac{2 K|r(t, x, \alpha)|}{|x| \max \left\{-\beta_{1}(\alpha), \beta_{2}(\alpha)\right\}}<1 .
$$

Then, there exist $\hat{\alpha}_{-}<0<\hat{\alpha}_{+}$such that the following statements are fulfilled:

(i) In case (6.24) and the functions $\beta_{1}$ and $\beta_{2}$ are monotone increasing, the trivial solution is past (future, all-time, respectively) attractive for $\alpha \in\left(\hat{\alpha}_{-}, \alpha_{0}\right)$ and past (future, alltime, respectively) repulsive for $\alpha \in\left(\alpha_{0}, \hat{\alpha}_{+}\right)$. The differential equation (6.23) $\alpha$ admits a past (future, all-time, respectively) bifurcation, since the corresponding radii of past (future, all-time, respectively) attraction satisfy

$$
\lim _{\alpha>\alpha_{0}} \mathfrak{A}_{0}^{\alpha}=0 .
$$

If, in addition, $\square=\mathbb{R}_{\mathcal{K}}^{+}$is fulfilled, then, for $\alpha \in\left(\hat{\alpha}_{-}, \alpha_{0}\right)$, there exists a nontrivial future

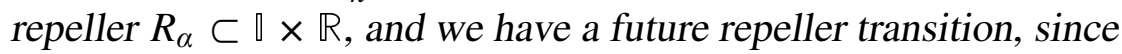

$$
\lim _{\alpha>\alpha_{0}} d_{H}\left(R_{\alpha}(t),\{0\}\right)=0 \quad \text { for all } t \in \mathbb{\square} .
$$

(ii) In case (6.25) and the functions $\beta_{1}$ and $\beta_{2}$ are monotone increasing, the trivial solution is all-time (past, future, respectively) attractive for $\alpha \in\left(\hat{\alpha}_{-}, \alpha_{0}\right)$ and past (future, alltime, respectively) repulsive for $\alpha \in\left(\alpha_{0}, \hat{\alpha}_{+}\right)$. The differential equation (6.23) $\alpha$ admits a past (future, all-time, respectively) bifurcation, since the corresponding radii of past (future, all-time, respectively) repulsion satisfy

$$
\lim _{\alpha \searrow \alpha_{0}} \mathfrak{R}_{0}^{\alpha}=0 .
$$

If, in addition, $\square=\mathbb{R}_{\kappa}^{-}$is fulfilled, then, for $\alpha \in\left(\alpha_{0}, \hat{\alpha}_{+}\right)$, there exists a nontrivial past 


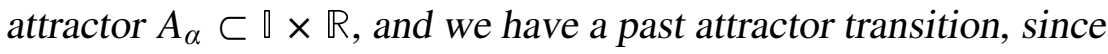

$$
\lim _{\alpha \unlhd \alpha_{0}} d_{H}\left(A_{\alpha}(t),\{0\}\right)=0 \quad \text { for all } t \in \mathbb{\square} .
$$

(iii) In case (6.24) and the functions $\beta_{1}$ and $\beta_{2}$ are monotone decreasing, the trivial solution is past (future, all-time, respectively) repulsive for $\alpha \in\left(\hat{\alpha}_{-}, \alpha_{0}\right)$ and past (future, alltime, respectively) attractive for $\alpha \in\left(\alpha_{0}, \hat{\alpha}_{+}\right)$. The differential equation (6.23) $)_{\alpha}$ admits a past (future, all-time, respectively) bifurcation, since the corresponding radii of past (future, all-time, respectively) attraction satisfy

$$
\lim _{\alpha \searrow \alpha_{0}} \mathfrak{A}_{0}^{\alpha}=0
$$

If, in addition, $\square=\mathbb{R}^{+}$is fulfilled, then, for $\alpha \in\left(\alpha_{0}, \hat{\alpha}_{+}\right)$, there exists a nontrivial future

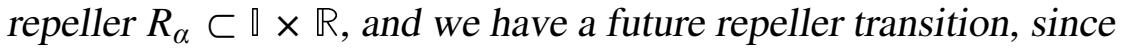

$$
\lim _{\alpha \searrow \alpha_{0}} d_{H}\left(R_{\alpha}(t),\{0\}\right)=0 \quad \text { for all } t \in \mathbb{\square} .
$$

(iv) In case (6.25) and the functions $\beta_{1}$ and $\beta_{2}$ are monotone decreasing, the trivial solution is past (future, all-time, respectively) repulsive for $\alpha \in\left(\hat{\alpha}_{-}, \alpha_{0}\right)$ and past (future, alltime, respectively) attractive for $\alpha \in\left(\alpha_{0}, \hat{\alpha}_{+}\right)$. The differential equation (6.23) $)_{\alpha}$ admits a past (future, all-time, respectively) bifurcation, since the corresponding radii of past (future, all-time, respectively) repulsion satisfy

$$
\lim _{\alpha>\alpha_{0}} \mathfrak{R}_{0}^{\alpha}=0
$$

If, in addition, $\square=\mathbb{R}^{-}$is fulfilled, then, for $\alpha \in\left(\hat{\alpha}_{-}, \alpha_{0}\right)$, there exists a nontrivial past

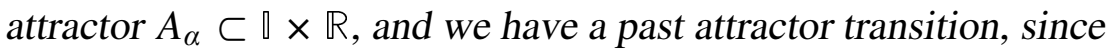

$$
\lim _{\alpha>\alpha_{0}} d_{H}\left(A_{\alpha}(t),\{0\}\right)=0 \quad \text { for all } t \in \mathbb{\square} .
$$

PROOF. The first part of this theorem concerning the bifurcation of the attraction or repulsion areas, respectively, can be proved using the same methods as in the proof of Theorem 6.1.1. We write $\tilde{\alpha}_{-}$and $\tilde{\alpha}_{+}$for the constants $\hat{\alpha}_{-}$and $\hat{\alpha}_{+}$used in this proof. For the proof of the attractor and repeller transitions, w.l.o.g, we only consider the case (ii), i.e., $\square=\mathbb{R}_{\kappa}^{-}$, condition (6.25) holds and the functions $\beta_{1}$ and $\beta_{2}$ are monotone increasing. We denote the general solution of $(6.23)_{\alpha}$ by $\lambda_{\alpha}$ and define

$$
b_{+}:=\frac{1}{2} \sup _{t \in \llbracket, \alpha \in\left(\tilde{\alpha}_{-}, \tilde{\alpha}_{+}\right)} b(t, \alpha)<0 .
$$

Due to (6.26), there exists a $\rho>0$ such that

$$
|r(t, x, \alpha)| \leq-b_{+}|x|^{3} \quad \text { for all } x \in[-\rho, \rho], \alpha \in\left(\alpha_{0}-x^{2}, \alpha_{0}+x^{2}\right) \text { and } t \in \mathbb{\square} .
$$

The remaining proof is divided into two steps.

STEP 1. For given $x_{1}, x_{2}, x_{3} \leq \rho$ such that $0<x_{1} \leq x_{2} \leq \frac{x_{3}}{2 K}$, there exists a uniquely determined constant

$$
\alpha^{*}=\alpha^{*}\left(x_{1}, x_{2}, x_{3}\right) \in\left(\alpha_{0}, \min \left\{\alpha_{0}+x_{1}^{2}, \tilde{\alpha}_{+}\right\}\right]
$$

with the following properties: 
- For all $\tau \leq t \leq \kappa$ and $\alpha \in\left(\alpha_{0}, \alpha^{*}\right)$, we have $\lambda_{\alpha}\left(t, \tau,\left[-x_{2}, x_{2}\right]\right) \subset\left(-x_{3}, x_{3}\right)$,

- there exists a constant $T^{*}>0$ such that for all $\alpha \in\left(\alpha_{0}, \alpha^{*}\right)$ and $\tau \leq \kappa-T^{*}$, there exist $t_{+}, t_{-} \in\left[0, T^{*}\right]$ with

$$
\lambda_{\alpha}\left(\tau+t_{+}, \tau, x_{2}\right)=x_{1} \quad \text { and } \quad \lambda_{\alpha}\left(\tau+t_{-}, \tau,-x_{2}\right)=-x_{1},
$$

- $\alpha^{*}$ is chosen maximal, i.e., for all bigger $\alpha^{*}$, one of the two above properties is violated.

We will only prove the existence of a constant $\alpha^{*}$ such that

(a) for all $\tau \leq t \leq \kappa$ and $\alpha \in\left(\alpha_{0}, \alpha^{*}\right)$, we have $\lambda_{\alpha}\left(t, \tau, x_{2}\right) \leq x_{3}$,

(b) there exists a constant $T^{*}>0$ such that for all $\alpha \in\left(\alpha_{0}, \alpha^{*}\right)$ and $\tau \leq \kappa-T^{*}$, there exists a $t_{+} \in\left[0, T^{*}\right]$ with $\lambda_{\alpha}\left(\tau+t_{+}, \tau, x_{2}\right)=x_{1}$,

since the extension to the above assertion follows similarly and by taking the supremum of all such $\alpha^{*}$. We first note that for arbitrary $\tau \in \mathbb{\mathbb { V }}$, the solution $\mu_{\tau}(\cdot):=\lambda_{\alpha}\left(\cdot, \tau, x_{2}\right)$ of $(6.23)_{\alpha}$ is also a solution of the inhomogeneous linear differential equation

$$
\dot{x}=a(t, \alpha) x+b(t, \alpha)\left(\mu_{\tau}(t)\right)^{3}+r\left(t, \mu_{\tau}(t), \alpha\right) .
$$

Concerning the expression

$$
s(\alpha, T):=K e^{\beta_{1}(\alpha) T} x_{2}+\frac{b_{+} x_{1}^{3}}{K} T \text { for all } \alpha \in\left(\alpha_{0}, \tilde{\alpha}_{+}\right) \text {and } T \geq 0,
$$

there exist $\alpha^{*} \in\left(\alpha_{0}, \min \left\{\alpha_{0}+x_{1}^{2}, \tilde{\alpha}_{+}\right\}\right]$and $T^{*}>0$ such that for all $\alpha \in\left(\alpha_{0}, \alpha^{*}\right]$, we have

$$
s\left(\alpha, T^{*}\right)<0 \quad \text { and } \quad s(\alpha, T) \leq 2 K x_{2} \text { for all } T \in\left[0, T^{*}\right] .
$$

This follows by choosing $T^{*}$ such that $\frac{b_{+} x_{1}^{3}}{K} T^{*} \leq-2 K x_{2}$ and $\alpha^{*}$ such that $\exp \left(\beta_{1}\left(\alpha^{*}\right) T^{*}\right) \leq 2$. Choose $\alpha \in\left(\alpha_{0}, \alpha^{*}\right]$ and $\tau, \tau^{*} \leq \kappa$ with $\tau \leq \tau^{*}$. Assume that $x_{1} \leq \mu_{\tau}(t) \leq x_{3}$ for all $t \in\left[\tau, \tau^{*}\right]$. Then, the variation of constants formula (cf. Proposition A.1.6), applied to (6.27), yields the relation

$$
\begin{aligned}
\mu_{\tau}\left(\tau^{*}\right) & =\Lambda_{\alpha}\left(\tau^{*}, \tau\right) x_{2}+\int_{\tau}^{\tau^{*}} \underbrace{\Lambda_{\alpha}\left(\tau^{*}, s\right)}_{\geq \frac{1}{K} \exp \left(\beta_{2}(\alpha)\left(\tau^{*}-s\right)\right)}(\underbrace{b(s, \alpha)\left(\mu_{\tau}(s)\right)^{3}+r\left(s, \mu_{\tau}(s), \alpha\right)}_{\leq b_{+} x_{1}^{3}<0}) d s \\
& \leq K e^{\beta_{1}(\alpha)\left(\tau^{*}-\tau\right)} x_{2}+\int_{\tau}^{\tau^{*}} \frac{1}{K} e^{\beta_{2}(\alpha)\left(\tau^{*}-s\right)} b_{+} x_{1}^{3} d s \\
& =K e^{\beta_{1}(\alpha)\left(\tau^{*}-\tau\right)} x_{2}+\frac{b_{+} x_{1}^{3}}{K \beta_{2}(\alpha)}\left(e^{\beta_{2}(\alpha)\left(\tau^{*}-\tau\right)}-1\right) \\
& \leq K e^{\beta_{1}(\alpha)\left(\tau^{*}-\tau\right)} x_{2}+\frac{b_{+} x_{1}^{3}}{K}\left(\tau^{*}-\tau\right)=s\left(\alpha, \tau^{*}-\tau\right) .
\end{aligned}
$$

Since $s(\alpha, T) \leq 2 K x_{2} \leq x_{3}$ for all $T \in\left[0, T^{*}\right]$, the assumption $\mu_{\tau}(t) \leq x_{3}$ for all $t \in\left[\tau, \tau^{*}\right]$ is justified. This proves (a). Because of $s\left(\alpha, T^{*}\right)<0$, also (b) is fulfilled.

STEP 2. There exists a $\hat{\alpha}_{+} \in\left(\alpha_{0}, \tilde{\alpha}_{+}\right)$such that for all $\alpha \in\left(\alpha_{0}, \hat{\alpha}_{+}\right)$, there exists a nontrivial past

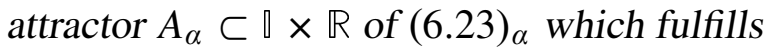

$$
\lim _{\alpha \searrow \alpha_{0}} d_{H}\left(A_{\alpha}(t),\{0\}\right)=0 \quad \text { for all } t \in \mathbb{\square} .
$$


For $x_{3}:=\frac{\rho}{K}$ and $x_{2}:=\frac{x_{3}}{2 K}$, we consider the function $\gamma:\left(0, x_{2}\right) \rightarrow\left(\alpha_{0}, \alpha_{+}\right)$, defined by

$$
\gamma\left(x_{1}\right):=\alpha^{*}\left(x_{1}, x_{2}, x_{3}\right) \text { for all } x_{1} \in\left(0, x_{2}\right),
$$

where $\alpha^{*}$ stems from Step 1 . We set $\bar{\alpha}:=\gamma\left(\frac{x_{2}}{2}\right)$ and define

$$
\delta(\alpha):=\inf \left\{x_{1} \in\left(0, x_{2}\right): \gamma\left(x_{1}\right) \geq \alpha\right\} \text { for all } \alpha \in\left(\alpha_{0}, \bar{\alpha}\right] .
$$

Due to $\alpha_{0}<\alpha^{*}\left(x_{1}, x_{2}, x_{3}\right) \leq \alpha_{0}+x_{1}^{2}$, we have $\lim _{x_{1} \rightarrow 0} \gamma\left(x_{1}\right)=\alpha_{0}$, and since $\gamma$ is monotone increasing, this implies that $\delta$ is monotone increasing, $\delta(\alpha)>0$ for all $\alpha \in\left(\alpha_{0}, \bar{\alpha}\right]$ and

$$
\lim _{\alpha \searrow \alpha_{0}} \delta(\alpha)=0
$$

We define

$$
\bar{x}_{3}(\alpha):=3 K \delta(\alpha) \quad \text { and } \quad \bar{x}_{2}(\alpha):=\bar{x}_{1}(\alpha):=\frac{3}{2} \delta(\alpha) \quad \text { for all } \alpha \in\left(\alpha_{0}, \bar{\alpha}\right]
$$

and consider the function $\bar{\gamma}:\left(\alpha_{0}, \bar{\alpha}\right] \rightarrow\left(\alpha_{0}, \alpha_{+}\right)$, defined by

$$
\bar{\gamma}(\alpha):=\alpha^{*}\left(\bar{x}_{1}(\alpha), \bar{x}_{2}(\alpha), \bar{x}_{3}(\alpha)\right) \text { for all } \alpha \in\left(\alpha_{0}, \bar{\alpha}\right]
$$

where $\alpha^{*}$ is taken from Step 1 again. Moreover, we define

$$
M:=\left[-x_{2}, x_{2}\right] \text { and } B_{\alpha}:=\left[-\bar{x}_{3}(\alpha), \bar{x}_{3}(\alpha)\right] \text { for all } \alpha \in\left(\alpha_{0}, \bar{\alpha}\right]
$$

and fix a $\beta \in\left(\alpha_{0}, \bar{\alpha}\right]$ and an $\alpha \in\left(\alpha_{0}, \min \{\bar{\gamma}(\beta), \beta\}\right)$. Since $\alpha \leq \beta$ and $x_{2}>\frac{3}{2} \delta(\alpha)$ and due to the definition of $\delta$, there exists a $T^{*}>0$ such that for all $\tau \leq \kappa-T^{*}$, there exist $t_{+}, t_{-} \in\left[0, T^{*}\right]$ with

$$
\lambda_{\alpha}\left(t^{+}, \tau, x_{2}\right)=\frac{3}{2} \delta(\beta)=\bar{x}_{2}(\beta) \quad \text { and } \quad \lambda_{\alpha}\left(t^{-}, \tau,-x_{2}\right)=-\frac{3}{2} \delta(\beta)=-\bar{x}_{2}(\beta) .
$$

Moreover, since $\alpha<\bar{\gamma}(\beta)$, for all $\tau \leq t \leq \kappa$, we have

$$
\lambda_{\alpha}\left(t, \tau,\left[-\bar{x}_{2}(\beta), \bar{x}_{2}(\beta)\right]\right) \subset\left(-\bar{x}_{3}(\beta), \bar{x}_{3}(\beta)\right) .
$$

This means that, considering equation $(6.23)_{\alpha}, B_{\beta} \times \square$ is past absorbing with respect to $\{M \times \square\}$. Then, due to Theorem 2.1.25 (i), there exists a past attractor $A_{\alpha} \subset B_{\beta} \times \mathbb{~}$. The past attractor is nontrivial due to Lemma 2.1.29. The limit relation

$$
\lim _{\alpha>\alpha_{0}} d_{H}\left(A_{\alpha}(t),\{0\}\right)=0 \quad \text { for all } t \in \mathbb{0}
$$

follows from $A_{\alpha} \subset B_{\beta} \times \llbracket$ for all $\alpha<\min \{\bar{\gamma}(\beta), \beta\}$ and (6.28). By setting $\hat{\alpha}_{+}:=\bar{\gamma}(\bar{\alpha})$, all assertions of this theorem are proved.

\subsubsection{REMARKS.}

(i) In the limit $\alpha \rightarrow \alpha_{0}$, the attractivity or repulsivity of the trivial solution is lost in both directions, i.e., no situation as described in Remark 6.1.2 (i) can occur. This means that nonautonomous pitchfork bifurcations are total bifurcations. 
(ii) The Hypothesis on the linear part implies that the past (future, all-time, respectively) dichotomy spectrum of the linearization $\dot{x}=a(t, \alpha) x$ converges to $\{0\}$ in Hausdorff distance in the limit $\alpha \rightarrow \alpha_{0}$.

(iii) As in Example 6.1.3, one can show that Theorem 6.2.1 is a proper generalization of the well-known autonomous pitchfork bifurcation (see, e.g., Wiggins [181, p. 267 f.] and Aulbach [14, Satz 7.10.8]).

(iv) Please note that the above bifurcation result is essentially the combination of two scenarios which are independent of each other. This means that it is possible to consider $(6.23)_{\alpha}$ only for $\alpha>\alpha_{0}$ or $\alpha<\alpha_{0}$, respectively, in order to obtain the results which apply for these parameter values.

6.2.3 Theorem (Nonautonomous Pitchfork Bifurcation, Part II). Let $x_{-}<$

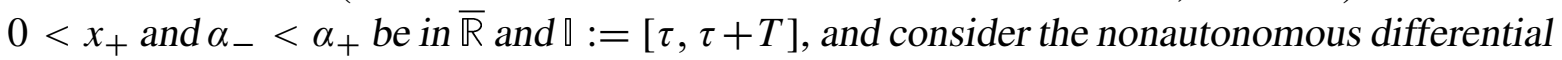
equation

$$
\dot{x}=a(t, \alpha) x+b(t, \alpha) x^{3}+r(t, x, \alpha)
$$

with continuous functions $a: \llbracket \times\left(\alpha_{-}, \alpha_{+}\right) \rightarrow \mathbb{R}, b: \square \times\left(\alpha_{-}, \alpha_{+}\right) \rightarrow \mathbb{R}$ and $r: \llbracket \times\left(x_{-}, x_{+}\right) \times$

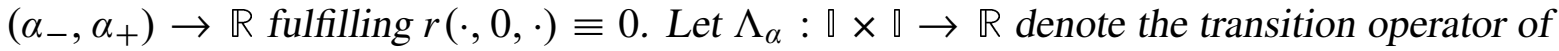
the linearized equation $\dot{x}=a(t, \alpha) x$. We define

$$
K(\alpha):=\sup \left\{\Lambda_{\alpha}(t, s): t, s \in \mathbb{\text { for all }} \alpha \in\left(\alpha_{-}, \alpha_{+}\right)\right.
$$

and assume, there exists an $\alpha_{0} \in\left(\alpha_{-}, \alpha_{+}\right)$such that the following hypotheses hold:

- HYPOTHESIS ON LINEAR PART. We either have

$$
\begin{array}{ll}
\Lambda_{\alpha}(\tau+T, \tau)<1 & \text { for all } \alpha \in\left(\alpha_{-}, \alpha_{0}\right) \text { and } \\
\Lambda_{\alpha}(\tau+T, \tau)>1 & \text { for all } \alpha \in\left(\alpha_{0}, \alpha_{+}\right)
\end{array}
$$

or

$$
\begin{array}{ll}
\Lambda_{\alpha}(\tau+T, \tau)>1 & \text { for all } \alpha \in\left(\alpha_{-}, \alpha_{0}\right) \text { and } \\
\Lambda_{\alpha}(\tau+T, \tau)<1 & \text { for all } \alpha \in\left(\alpha_{0}, \alpha_{+}\right) .
\end{array}
$$

- HyPOTHESIS ON NONLINEARITY. The cubic term either fulfills

$$
\liminf _{\alpha \rightarrow \alpha_{0}} \inf _{t \in \mathbb{\prod}} b(t, \alpha)>0
$$

or

$$
\limsup _{\alpha \rightarrow \alpha_{0}} \sup _{t \in \rrbracket} b(t, \alpha)<0,
$$

and the remainder satisfies

$$
\lim _{x \rightarrow 0} \sup _{\alpha \in\left(\alpha_{0}-x^{2}, \alpha_{0}+x^{2}\right)} \sup _{t \in \mathbb{\complement}} \frac{|r(t, x, \alpha)|}{|x|^{3}}=0
$$

and

$$
\limsup _{\alpha \rightarrow \alpha_{0}} \limsup _{x \rightarrow 0} \sup _{t \in \mathbb{0}}-\frac{T K(\alpha)|r(t, x, \alpha)|}{|x| \ln \left(\min \left\{\Lambda_{\alpha}(\tau+T, \tau), \Lambda_{\alpha}(\tau, \tau+T)\right\}\right)}<1 .
$$


Then, there exist $\hat{\alpha}_{-}<0<\hat{\alpha}_{+}$such that the following statements are fulfilled:

(i) In case (6.30) and (6.32) is fulfilled, the trivial solution is $(\tau, T)$-attractive for $\alpha \in\left(\hat{\alpha}_{-}, \alpha_{0}\right)$ and $(\tau, T)$-repulsive for $\alpha \in\left(\alpha_{0}, \hat{\alpha}_{+}\right)$. The differential equation $(6.29)_{\alpha}$ admits a $(\tau, T)$-bifurcation, since the corresponding radii of $(\tau, T)$-attraction satisfy

$$
\lim _{\alpha>\alpha_{0}} \mathfrak{A}_{0}^{\alpha}=0
$$

(ii) In case (6.30) and (6.33) is fulfilled, the trivial solution is $(\tau, T)$-attractive for $\alpha \in\left(\hat{\alpha}_{-}, \alpha_{0}\right)$ and $(\tau, T)$-repulsive for $\alpha \in\left(\alpha_{0}, \hat{\alpha}_{+}\right)$. The differential equation $(6.29)_{\alpha}$ admits a $(\tau, T)$-bifurcation, since the corresponding radii of $(\tau, T)$-repulsion satisfy

$$
\lim _{\alpha \triangleright \alpha_{0}} \mathfrak{R}_{0}^{\alpha}=0 .
$$

(iii) In case (6.31) and (6.32) is fulfilled, the trivial solution is $(\tau, T)$-repulsive for $\alpha \in\left(\hat{\alpha}_{-}, \alpha_{0}\right)$ and $(\tau, T)$-attractive for $\alpha \in\left(\alpha_{0}, \hat{\alpha}_{+}\right)$. The differential equation (6.29) $\alpha$ admits a $(\tau, T)$-bifurcation, since the corresponding radii of $(\tau, T)$-attraction satisfy

$$
\lim _{\alpha \triangleleft \alpha_{0}} \mathfrak{A}_{0}^{\alpha}=0
$$

(iv) In case (6.31) and (6.33) is fulfilled, the trivial solution is $(\tau, T)$-repulsive for $\alpha \in\left(\hat{\alpha}_{-}, \alpha_{0}\right)$ and $(\tau, T)$-attractive for $\alpha \in\left(\alpha_{0}, \hat{\alpha}_{+}\right)$. The differential equation (6.29) $\alpha$ admits a $(\tau, T)$-bifurcation, since the corresponding radii of $(\tau, T)$-repulsion satisfy

$$
\lim _{\alpha>\alpha_{0}} \mathfrak{R}_{0}^{\alpha}=0
$$

PROOF. This theorem can be proved using the same methods as in the proof of Theorem 6.2.1.

\subsubsection{REMARKS.}

(i) The Hypothesis on the linear part implies that the $(\tau, T)$-dichotomy spectrum of the linearization $\dot{x}=a(t, \alpha) x$ converges to $\{0\}$ in Hausdorff distance in the limit $\alpha \rightarrow \alpha_{0}$.

(ii) Condition (6.35) is only used to obtain the attractivity or repulsivity of the trivial solution by applying Theorem 5.3.2. Alternatively, one can directly postulate that the trivial solution changes their stability at the parameter value $\alpha_{0}$ from, say, attractivity to repulsivity. 



\section{CHAPTER 7}

\section{BIFURCATIONS OF ASYMPTOTICALLY AUTONOMOUS SYSTEMS}

A nonautonomous differential equation

$$
\dot{x}=f(t, x)
$$

is called past (future, respectively) asymptotically autonomous with limiting equation

$$
\dot{x}=g(x)
$$

if $\lim _{t \rightarrow-\infty} f(t, x)=g(x)\left(\lim _{t \rightarrow \infty} f(t, x)=g(x)\right.$, respectively) holds uniformly for every element $x$ of the domain of the function $g$. This chapter deals with the question of transferring bifurcation phenomena from the autonomous differential equation (7.2) to the nonautonomous differential equation (7.1).

The study of asymptotically autonomous differential equations goes back to MARKUS [114]. Markus discusses properties of nonautonomous $\omega$-limit sets and generalizes the THEOREM OF Poincaré \& Bendixson (see, e.g., Palis \& De Melo [123] and Hirsch \& Smale [80, Chapter 11]) to asymptotically autonomous planar systems. His work has stimulated the qualitative theory of nonautonomous differential equations (see, e.g., SELL $[164,165,166])$. Further fundamental work on asymptotically autonomous systems was achieved by STRAUSS \& YORKE [177], Artstein [10, 11], Thieme [178] and Mischaikow \& Smith \& Thieme [120] in the context of differential equations (see also KATO \& MARTYNYUK \& SHESTAKOV [89]); for difference equations, we refer to SCHÖNEFUSS [162].

It is not clear a priori under which assumptions certain behavior carries over from the autonomous to the nonautonomous system. In fact, in THIEME [178], several examples of asymptotically autonomous systems are studied that behave quite differently from the limiting equations. In LANGA \& ROBINSON \& SUÁREZ [103], however, it is shown that the pullback and forward behavior of a special asymptotically autonomous Lotka-Volterra system is consistent to the underlying autonomous system.

In the first section of this chapter, some basic properties of asymptotically autonomous systems are prepared for later use. In Section 7.2, one-dimensional bifurcations such as the pitchfork, transcritical and saddle node bifurcation are discussed. Section 7.3 is devoted to study the Hopf bifurcation scenario.

Whenever considering a nonautonomous differential equation in this chapter, its general solution is denoted by $\lambda$. For the flow of an autonomous differential equation, we write $\phi$. 


\subsection{Basic Properties of As ymptotically Autonomous SYSTEMS}

In this section, some useful lemmata are derived for asymptotically autonomous differential equations. The first two lemmata deal with the question of controlling the distances of the time evolutions of both systems on compact time intervals.

7.1.1 Lemma. Consider an open set $D \subset \mathbb{R}^{N}$, a nonautonomous differential equation

$$
\dot{x}=f(t, x)
$$

with a $C^{1}$-function $f:(-\infty, 0) \times D \rightarrow \mathbb{R}^{N}$ and an autonomous differential equation

$$
\dot{x}=g(x)
$$

with a $C^{1}$-function $g: D \rightarrow \mathbb{R}^{N}$. We assume that

$$
\lim _{t \rightarrow-\infty} f(t, x)=g(x) \quad \text { uniformly for } x \in D .
$$

Furthermore, let $K \subset D$ be a compact and convex set. Then, the following statements are fulfilled:

(i) For all $T>0$ and $\varepsilon>0$, there exists a $\tau_{0}<-T$ such that for all $T^{\prime} \leq T$ and $x \in K$ with

$$
\phi(t, x) \in K \quad \text { for all } t \in\left[0, T^{\prime}\right]
$$

the relation

$$
\|\lambda(\tau+t, \tau, x)-\phi(t, x)\| \leq \varepsilon \quad \text { for all } \tau \leq \tau_{0} \text { and } t \in\left[0, T^{\prime}\right]
$$

is fulfilled.

(ii) For all $T>0$ and $\varepsilon>0$, there exists a $\tau_{0}<0$ such that for all $T^{\prime} \leq T$ and $x \in K$ with

$$
\phi(-t, x) \in K \quad \text { for all } t \in\left[0, T^{\prime}\right],
$$

the relation

$$
\|\lambda(\tau-t, \tau, x)-\phi(-t, x)\| \leq \varepsilon \quad \text { for all } \tau \leq \tau_{0} \text { and } t \in\left[0, T^{\prime}\right]
$$

is fulfilled.

Proof. (i) Since $D$ is open, there exist a compact and convex set $\tilde{K}$ and an $\eta>0$ such that $U_{\eta}(K) \subset \tilde{K}$. We choose $T>0$ and $\varepsilon>0$ arbitrarily and define $M:=\max _{x \in \tilde{K}}\|D g(x)\|$. Due to (7.3), there exists a $\tau_{0}<-T$ with

$$
\|f(t+T, x)-g(x)\| \leq \frac{\min \{\varepsilon, \eta\}}{T e^{M T}} \quad \text { for all } t \leq \tau_{0} \text { and } x \in D .
$$

For the rest of this proof, we fix arbitrary numbers $\tau \leq \tau_{0}, T^{\prime} \leq T$ and $x \in K$ fulfilling

$$
\phi(t, x) \in K \quad \text { for all } t \in\left[0, T^{\prime}\right] .
$$


Since

$$
\lambda(t+\tau, \tau, x)-\phi(t, x)=\int_{0}^{t}(f(s+\tau, \lambda(s+\tau, \tau, x))-g(\phi(s, x))) d s \quad \text { for all } t \in\left[0, T^{\prime}\right]
$$

it follows from the mean value inequality (see, e.g., ABRAHAM \& MARSDEN \& RATIU [1, Theorem 2.4 .8$, p. 87]) that

$$
\begin{aligned}
& \|\lambda(t+\tau, \tau, x)-\phi(t, x)\| \\
\leq & \int_{0}^{t}\|f(s+\tau, \lambda(s+\tau, \tau, x))-g(\phi(s, x))\| d s \\
\leq & \int_{0}^{t}(\|f(s+\tau, \lambda(s+\tau, \tau, x))-g(\lambda(s+\tau, \tau, x))\|+\|g(\lambda(s+\tau, \tau, x))-g(\phi(s, x))\|) d s \\
\leq & \frac{t \min \{\varepsilon, \eta\}}{T e^{M T}}+M \int_{0}^{t}\|\lambda(s+\tau, \tau, x)-\phi(s, x)\| d s .
\end{aligned}
$$

Assume, there exists a $t \in\left(0, T^{\prime}\right)$ with $\|\lambda(t+\tau, \tau, x)-\phi(t, x)\| \geq \min \{\varepsilon, \eta\}$. We define

$$
T^{*}:=\min \left\{t \in\left(0, T^{\prime}\right):\|\lambda(t+\tau, \tau, x)-\phi(t, x)\| \geq \min \{\varepsilon, \eta\}\right\}<T^{\prime} .
$$

Hence, from Gronwall's inequality (Lemma A.2.1), we obtain

$$
\left\|\lambda\left(T^{*}+\tau, \tau, x\right)-\phi\left(T^{*}, x\right)\right\| \leq \frac{T^{*} \min \{\varepsilon, \eta\}}{T e^{M T}} e^{M T^{*}}<\min \{\varepsilon, \eta\} .
$$

This is a contradiction and finishes the proof of this lemma.

(ii) See proof of (i).

7.1.2 LEMmA. Consider an open set $D \subset \mathbb{R}^{N}$, a nonautonomous differential equation

$$
\dot{x}=f(t, x)
$$

with a $C^{1}$-function $f:(0, \infty) \times D \rightarrow \mathbb{R}^{N}$ and an autonomous differential equation

$$
\dot{x}=g(x)
$$

with a $C^{1}$-function $g: D \rightarrow \mathbb{R}^{N}$. We assume that

$$
\lim _{t \rightarrow \infty} f(t, x)=g(x) \quad \text { uniformly for } x \in D
$$

Furthermore, let $K \subset D$ be a compact and convex set. Then, the following statements are fulfilled:

(i) For all $T>0$ and $\varepsilon>0$, there exists a $\tau_{0}>0$ such that for all $T^{\prime} \leq T$ and $x \in K$ with

$$
\phi(t, x) \in K \quad \text { for all } t \in\left[0, T^{\prime}\right],
$$

the relation

$$
\|\lambda(\tau+t, \tau, x)-\phi(t, x)\| \leq \varepsilon \quad \text { for all } \tau \geq \tau_{0} \text { and } t \in\left[0, T^{\prime}\right]
$$

is fulfilled. 
(ii) For all $T>0$ and $\varepsilon>0$, there exists a $\tau_{0}>T$ such that for all $T^{\prime} \leq T$ and $x \in K$ with

$$
\phi(-t, x) \in K \quad \text { for all } t \in\left[0, T^{\prime}\right],
$$

the relation

$$
\|\lambda(\tau-t, \tau, x)-\phi(t, x)\| \leq \varepsilon \quad \text { for all } \tau \geq \tau_{0} \text { and } t \in\left[0, T^{\prime}\right]
$$

is fulfilled.

PROOF. See proof of Lemma 7.1.1.

In case of the classical autonomous bifurcations for ODEs (such as pitchfork, transcritical, saddle node and Hopf bifurcation), after the bifurcation, the phase space can be separated into three invariant parts. Therefore, we restrict attention to the following situation: Let $D \subset \mathbb{R}^{N}$ be an open and convex set and

$$
\dot{x}=g(x)
$$

be an autonomous differential equation with a $C^{1}$-function $g: D \rightarrow \mathbb{R}^{N}$. We suppose that $D$ is the disjoint union of

- a bounded and open set $S^{i}$ (inner area),

- an open set $S^{o}$ (outer area),

- a compact set $S=\partial S^{i}=\partial S^{o}$ with int $S=\emptyset$.

(a)

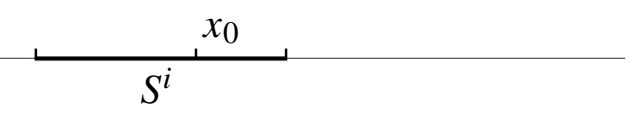

(b)

$$
S^{i}
$$

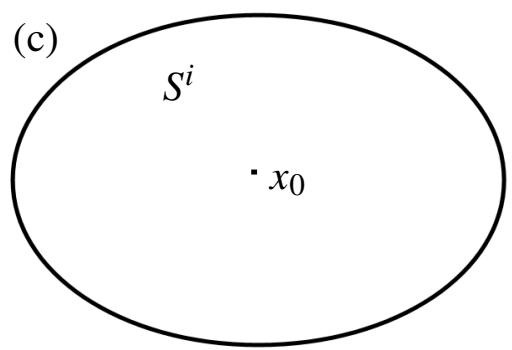

FIGURE 7.1. The above situation in case of a (a) pitchfork bifurcation, (b) transcritical or saddle node bifurcation, (c) Hopf bifurcation.

The occurrence of one of the above mentioned autonomous bifurcations means that (exactly) one of the following two hypotheses holds:

- Hypothesis $\left(\mathrm{H}_{1}\right)$. The following conditions are fulfilled:

(i) The inner area $S^{i}$ is forward invariant, i.e.,

$$
\phi(t, x) \in S^{i} \quad \text { for all } t \geq 0 \text { and } x \in S^{i},
$$

and there exists an attractive equilibrium $x_{0} \in S^{i}$ such that for all compact sets $K \subset S^{i}$, we have

$$
\lim _{t \rightarrow \infty} d\left(\phi(t, K) \mid\left\{x_{0}\right\}\right)=0 .
$$

(ii) The outer area $S^{o}$ is backward invariant, i.e.,

$$
\phi(t, x) \in S^{o} \quad \text { for all } t \leq 0 \text { and } x \in S^{o},
$$


and $S$ is a repeller, i.e., there exists an $\eta>0$ with

$$
\lim _{t \rightarrow \infty} d\left(\phi\left(-t, U_{\eta}(S)\right) \mid S\right)=0
$$

(iii) $S$ is invariant, i.e.,

$$
\phi(t, x) \in S \quad \text { for all } t \in \mathbb{R} \text { and } x \in S .
$$

- Hypothesis $\left(\mathrm{H}_{2}\right)$. The following conditions are fulfilled:

(i) The inner area $S^{i}$ is backward invariant, and there exists a repulsive equilibrium $x_{0} \in S^{i}$ such that for all compact sets $K \subset S^{i}$, we have

$$
\lim _{t \rightarrow \infty} d\left(\phi(-t, K) \mid\left\{x_{0}\right\}\right)=0 .
$$

(ii) The outer area $S^{o}$ is forward invariant, and $S$ is an attractor, i.e., there exists an $\eta>0$ with

$$
\lim _{t \rightarrow \infty} d\left(\phi\left(t, U_{\eta}(S)\right) \mid S\right)=0
$$

(iii) $S$ is invariant.

Now, some easy consequences of Hypothesis $\left(\mathrm{H}_{1}\right)$ are derived.

7.1.3 LEMMA. Under Hypothesis $\left(H_{1}\right)$, the following statements hold:

(i) For all $\delta>0$, there exists a $T>0$ such that for all $x \in D$ with $d(x, S) \geq \delta$, there exists a $\hat{T} \in[0, T]$ with

$$
d(\phi(\hat{T}, x), S) \geq \eta
$$

(ii) For all $\gamma>0$, there exists a $\delta>0$ with

$$
\phi\left(-t, U_{\delta}\left(S^{i}\right)\right) \subset U_{\gamma}\left(S^{i}\right) \text { for all } t \geq 0 .
$$

Proof. (i) We choose $\delta>0$ arbitrarily. Due to the hypotheses, there exists a $T>0$ with

$$
\phi\left(-T, U_{\eta}(S)\right) \subset U_{\frac{\delta}{2}}(S) .
$$

This implies the assertion.

(ii) We choose $\gamma>0$ arbitrarily. Since $S$ is repulsive and $S=\partial S^{i}$, there exists a $T>0$ with

$$
\phi\left(-t, U_{\eta}\left(S^{i}\right)\right) \subset U_{\gamma}\left(S^{i}\right) \text { for all } t>T .
$$

Arguing negatively, we assume that for all $n \in \mathbb{N}$, there exist $t_{n} \in[0, T]$ and $x_{n} \in U_{\frac{1}{n}}\left(S^{i}\right)$ with

$$
d\left(\phi\left(-t_{n}, x_{n}\right), S^{i}\right)=d\left(\phi\left(-t_{n}, x_{n}\right), S\right) \geq \gamma .
$$

Since $S$ is compact, we assume w.l.o.g. that the sequence $\left\{x_{n}\right\}_{n \in \mathbb{N}}$ is convergent with limit $x \in S$. Due to the continuity of the flow $\phi$ and the invariance of $S$, there exists a $\beta>0$ such that for all $y \in U_{\beta}(x)$ and $t \in[0, T]$, we have

$$
d(\phi(-t, y), S)<\frac{\gamma}{2} .
$$

This is a contradiction and finishes the proof of this lemma. 
7.1.4 REMARK. In case the outer area is forward invariant, statement (i) of the above lemma can be simplified as follows: For all $\delta>0$, there exists a $T>0$ such that for all $x \in D$ with $d(x, S) \geq \delta$,

$$
d(\phi(T, x), S) \geq \eta
$$

is fulfilled

The following two lemmata deal with the question of determining past (future, respectively) attraction areas of past (future, respectively) attractive solutions.

7.1.5 Lemma (Attraction Areas of Past Attractive Solutions). We suppose that Hypothesis $\left(\mathrm{H}_{1}\right)$ is fulfilled and consider the nonautonomous differential equation

$$
\dot{x}=f(t, x)
$$

with a $C^{1}$-function $f:(-\infty, 0) \times D \rightarrow \mathbb{R}^{N}$ such that

$$
\lim _{t \rightarrow-\infty} f(t, x)=g(x) \quad \text { uniformly for all } x \in D .
$$

Furthermore, for some $\tau<0$, let $\mu:(-\infty, \tau) \rightarrow \mathbb{R}^{N}$ be a past attractive solution of (7.4) with $\lim _{t \rightarrow-\infty} \mu(t)=x_{0}$. Then, we have

$$
\mathcal{A}_{\mu}^{\leftarrow}=S^{i}-x_{0}
$$

If, in addition, $S^{i}$ is bounded, then there exist $s<\tau$ and a past repeller $R \subset(-\infty, s) \times D$ with

$$
S^{i} \subset \liminf _{t \rightarrow-\infty} R(t) \subset \limsup _{t \rightarrow-\infty} R(t) \subset \operatorname{cls} S^{i}
$$

PROOF. The proof of this lemma is divided into four steps.

STEP $1 . \mathcal{A}_{\mu}^{\leftarrow} \supset S^{i}-x_{0}$

Since $\mu$ is past attractive, there exists a $\gamma>0$ such that for all $s<\tau$, we have

$$
\lim _{t \rightarrow-\infty} d\left(\lambda\left(s, t, U_{\gamma}(\mu(t))\right) \mid \mu(s)\right)=0 .
$$

We choose $y \in S^{i}$ arbitrarily. Let $C$ be a neighborhood of $y$ such that there exists a $\delta>0$ with cls $U_{\delta}(C) \subset S^{i}$. Since $\lim _{t \rightarrow-\infty} \mu(t)=x_{0}$, there exists a $t_{1}<\tau$ such that

$$
\mu(t) \in U_{\min }\left\{\frac{\gamma}{3}, \delta\right\}\left(x_{0}\right) \text { for all } t \leq t_{1} .
$$

Due to the attractivity of $x_{0}$, there exists a $T>0$ such that

$$
d\left(\phi\left(T, U_{\delta}(C)\right) \mid\left\{x_{0}\right\}\right)<\frac{\gamma}{3} .
$$

Since it is possible to choose a compact and convex superset $K \subset D$ of $S^{i}$ ( $D$ is convex), Lemma 7.1.1 (i) implies that there exists a $t_{2}<t_{1}-T$ with

$$
\|\lambda(t+T, t, x)-\phi(T, x)\| \leq \frac{\gamma}{3} \quad \text { for all } t \leq t_{2} \text { and } x \in \underbrace{\mu(t)+C-x_{0}}_{\subset U_{\delta}(C)} .
$$


Hence, for all $t \leq t_{2}$ and $x \in \mu(t)+C-x_{0}$, we have

$$
\begin{aligned}
& \|\lambda(t+T, t, x)-\mu(t+T)\| \\
\leq & \|\lambda(t+T, t, x)-\phi(T, x)\|+\left\|\phi(T, x)-x_{0}\right\|+\left\|x_{0}-\mu(t+T)\right\| \\
< & \frac{\gamma}{3}+\frac{\gamma}{3}+\frac{\gamma}{3}=\gamma .
\end{aligned}
$$

Thus,

$$
\begin{aligned}
& \lim _{t \rightarrow-\infty} d\left(\lambda\left(t_{2}, t, \mu(t)+C-x_{0}\right) \mid\left\{\mu\left(t_{2}\right)\right\}\right) \\
= & \lim _{t \rightarrow-\infty} d\left(\lambda(t_{2}, t+T, \underbrace{\lambda\left(t+T, t, \mu(t)+C-x_{0}\right)}_{\subset U_{\gamma}(\mu(t+T))}) \mid\left\{\mu\left(t_{2}\right)\right\}\right) \stackrel{(7.5)}{=} 0 .
\end{aligned}
$$

This implies $y-x_{0} \in \mathcal{A}_{\mu}^{\leftarrow}$, and since $y \in S^{i}$ has been chosen arbitrarily, we have $\mathcal{A}_{\mu}^{\leftarrow} \supset S^{i}-x_{0}$. STEP 2. $\mathcal{A}_{\mu}^{\leftarrow} \subset S^{i}-x_{0}$.

We choose $y \in S^{o}$ and $\beta>0$ such that $U_{\beta}\left(x_{0}\right) \subset S^{i}$ and define $\delta:=d(y, S)>0$. Due to Lemma 7.1.3 (i), there exists a $T>0$ such that for all $x \in S^{o}$ with $d(x, S) \geq \min \left\{\frac{\delta}{2}, \frac{\eta}{3}\right\}$, there exists a $\hat{T} \in[0, T]$ with

$$
d(\phi(\hat{T}, x), S) \geq \eta \quad \text { and } \quad \phi(\hat{T}, x) \in S^{o}
$$

Moreover, there exists a $t_{1}<\tau$ with

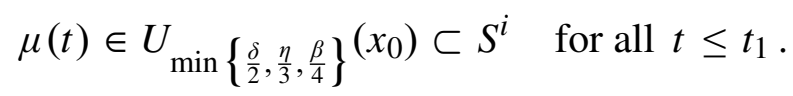

Let $K$ be a compact and convex superset of $U_{\eta}(S)$. Then, due to Lemma 7.1.1 (i), there exists a $t_{2}<t_{1}$ such that for all $\tilde{T} \in[0, T]$ and $x \in K$ with $\phi(t, x) \in K$ for all $t \in[0, \tilde{T}]$, we have

$$
\|\lambda(\hat{t}+t, \hat{t}, x)-\phi(t, x)\| \leq \min \left\{\frac{\beta}{2}, \frac{\eta}{3}\right\} \quad \text { for all } \hat{t} \leq t_{2} \text { and } t \in[0, \tilde{T}]
$$

We argue negatively and suppose that

$$
\lim _{t \rightarrow-\infty}\left\|\lambda\left(t_{2}, t, y-x_{0}+\mu(t)\right)-\mu\left(t_{2}\right)\right\|=0
$$

holds. Therefore, since $\mu\left(t_{2}\right) \in U_{\frac{\beta}{4}}\left(x_{0}\right)$, there exists a $t_{3}<t_{2}$ with

$$
\lambda\left(t_{2}, t_{3}, y-x_{0}+\mu\left(t_{3}\right)\right) \in U_{\frac{\beta}{2}}\left(x_{0}\right) .
$$

We define

$$
\begin{aligned}
& s:=\max \left\{t \in\left[t_{3}, t_{2}\right]: d\left(\lambda\left(t, t_{3}, y-x_{0}+\mu\left(t_{3}\right)\right), S\right) \geq \min \left\{\frac{\eta}{2}, \frac{\delta}{2}\right\}\right. \\
& \text { and } \left.\lambda\left(t, t_{3}, y-x_{0}+\mu\left(t_{3}\right)\right) \in S^{o}\right\} .
\end{aligned}
$$

This implies $d\left(\lambda\left(s, t_{3}, y-x_{0}+\mu\left(t_{3}\right)\right), S\right)=\min \left\{\frac{\eta}{2}, \frac{\delta}{2}\right\}$ and $\lambda\left(s, t_{3}, y-x_{0}+\mu\left(t_{3}\right)\right) \in S^{o}$.

We distinguish two cases. 
CASE $1 . t_{2}-s \leq T$.

CASE 1.1. For all $t \in\left[0, t_{2}-s\right]$, we have $\phi\left(t, \lambda\left(s, t_{3}, y-x_{0}+\mu\left(t_{3}\right)\right)\right) \in K$.

Due to (7.7), we have

$$
\left\|\phi\left(t, \lambda\left(s, t_{3}, y-x_{0}+\mu\left(t_{3}\right)\right)\right)-\lambda\left(t+s, t_{3}, y-x_{0}+\mu\left(t_{3}\right)\right)\right\| \leq \frac{\beta}{2} \quad \text { for all } t \in\left[0, t_{2}-s\right] .
$$

Since $\phi\left(t, \lambda\left(s, t_{3}, y-x_{0}+\mu\left(t_{3}\right)\right)\right) \in S^{o}$ for all $t \in\left[0, t_{2}-s\right]$, this leads to

$$
\begin{aligned}
& \left\|\lambda\left(t_{2}, t_{3}, y-x_{0}+\mu\left(t_{3}\right)\right)-x_{0}\right\| \\
\geq & \left\|\phi\left(t_{2}-s, \lambda\left(s, t_{3}, y-x_{0}+\mu\left(t_{3}\right)\right)\right)-x_{0}\right\|- \\
& \left\|\lambda\left(t_{2}, t_{3}, y-x_{0}+\mu\left(t_{3}\right)\right)-\phi\left(t_{2}-s, \lambda\left(s, t_{3}, y-x_{0}+\mu\left(t_{3}\right)\right)\right)\right\| \\
\geq & \beta-\frac{\beta}{2}=\frac{\beta}{2} .
\end{aligned}
$$

This is a contradiction to (7.8).

CASE 1.2. There exists a $\tilde{t} \in\left[0, t_{2}-s\right]$ with $\phi\left(\tilde{t}, \lambda\left(s, t_{3}, y-x_{0}+\mu\left(t_{3}\right)\right)\right) \notin K$.

By defining

$$
\hat{s}:=\inf \left\{t \in\left[0, t_{2}-s\right]: \phi\left(t, \lambda\left(s, t_{3}, y-x_{0}+\mu\left(t_{3}\right)\right)\right) \notin K\right\}>0,
$$

we obtain $d\left(\phi\left(\hat{s}, \lambda\left(s, t_{3}, y-x_{0}+\mu\left(t_{3}\right)\right)\right), S\right) \geq \eta$. Due to (7.7), the relation

$$
\left\|\lambda\left(\hat{s}+s, t_{3}, y-x_{0}+\mu\left(t_{3}\right)\right)-\phi\left(\hat{s}, \lambda\left(s, t_{3}, y-x_{0}+\mu\left(t_{3}\right)\right)\right)\right\| \leq \frac{\eta}{3}
$$

holds. Hence, we have $d\left(\lambda\left(\hat{s}+s, t_{3}, y-x_{0}+\mu\left(t_{3}\right)\right), S\right) \geq \frac{2 \eta}{3}$ and $\lambda\left(\hat{s}+s, t_{3}, y-x_{0}+\mu\left(t_{3}\right)\right) \in S^{o}$. This is a contradiction to the definition of $s$.

CASE 2. $t_{2}-s>T$.

CASE 2.1. For all $t \in[0, T]$, we have $\phi\left(t, \lambda\left(s, t_{3}, y-x_{0}+\mu\left(t_{3}\right)\right)\right) \in K$.

Because of (7.6) and $d\left(\lambda\left(s, t_{3}, y-x_{0}+\mu\left(t_{3}\right)\right), S\right)=\min \left\{\frac{\delta}{2}, \frac{\eta}{2}\right\} \geq \min \left\{\frac{\delta}{2}, \frac{\eta}{3}\right\}$, there exists a $\hat{T} \in[0, T]$ with

$$
d\left(\phi\left(\hat{T}, \lambda\left(s, t_{3}, y-x_{0}+\mu\left(t_{3}\right)\right)\right), S\right) \geq \eta
$$

and (7.7) yields

$$
\left\|\phi\left(\hat{T}, \lambda\left(s, t_{3}, y-x_{0}+\mu\left(t_{3}\right)\right)\right)-\lambda\left(\hat{T}+s, t_{3}, y-x_{0}+\mu\left(t_{3}\right)\right)\right\| \leq \frac{\eta}{3} .
$$

Together, this implies

$$
d\left(\lambda\left(\hat{T}+s, t_{3}, y-x_{0}+\mu\left(t_{3}\right)\right), S\right) \geq \frac{2}{3} \eta \quad \text { and } \quad \lambda\left(\hat{T}+s, t_{3}, y-x_{0}+\mu\left(t_{3}\right)\right) \in S^{o} .
$$

This is a contradiction to the definition of $s$.

CASE 2.2. There exists a $\tilde{t} \in[0, T]$ with $\phi\left(\tilde{t}, \lambda\left(s, t_{3}, y-x_{0}+\mu\left(t_{3}\right)\right)\right) \notin K$.

This case is treated analogously to Case 1.2 (by writing $T$ instead of $t_{2}-s$ ).

Consequently, we have $y-x_{0} \notin \mathcal{A}_{\mu}^{\leftarrow}$. This leads to the assertion, since $S=\partial S^{i}$, int $S=\emptyset$ and $\mathcal{A}_{\mu}^{\leftarrow}$ is open.

STEP 3. For all $\kappa \leq \eta$, there exist $T>0$ and $t_{1}<\tau$ such that for all $t_{2}<t_{1}$ and $t>T$, we have

$$
\lambda\left(t_{2}-t, t_{2}, U_{\frac{5 \kappa}{6}}\left(\mathcal{A}_{\mu}^{\leftarrow}+\mu\left(t_{2}\right)\right)\right) \subset U_{\frac{2 \kappa}{3}}\left(\mathcal{A}_{\mu}^{\leftarrow}+\mu\left(t_{2}-t\right)\right)
$$


We choose $\kappa \leq \eta$ arbitrarily. By applying Lemma 7.1.3 (ii), there exists a $\delta>0$ with

$$
\phi\left(-t, U_{\delta}\left(S^{i}\right)\right) \subset U_{\frac{\kappa}{4}}\left(S^{i}\right) \text { for all } t \geq 0 .
$$

Due to the repulsivity of $S$, there exists a $T>0$ with

$$
\phi\left(-t, U_{\kappa}\left(S^{i}\right)\right) \subset U_{\frac{\delta}{2}}\left(S^{i}\right) \text { for all } t>T .
$$

By choosing $K$ as a convex and compact superset of $\cup_{t \in[0, T]} \phi\left(-t, U_{\mathcal{K}}\left(S^{i}\right)\right)$, we can apply Lemma 7.1.2 (ii), and we therefore get a $t_{1}<\tau$ with

$$
\begin{aligned}
& \left\|\lambda\left(t_{2}-t, t_{2}, x\right)-\phi(-t, x)\right\| \leq \frac{\delta}{2} \quad \text { for all } x \in U_{\kappa}\left(S^{i}\right), t_{2}<t_{1} \text { and } t \in[0, T] \\
& \text { and } \quad\left\|\mu(t)-x_{0}\right\| \leq \frac{\kappa}{6} \quad \text { for all } t<t_{1} \text {. }
\end{aligned}
$$

Thus,

$$
\lambda\left(t_{2}-T, t_{2}, U_{\kappa}\left(S^{i}\right)\right) \subset U_{\delta}\left(S^{i}\right) \subset U_{\frac{\kappa}{4}}\left(S^{i}\right) \text { for all } t_{2}<t_{1}
$$

is fulfilled. Because of (7.9), this leads to

$$
\phi\left(-t, \lambda\left(t_{2}-T, t_{2}, U_{\kappa}\left(S^{i}\right)\right)\right) \subset U_{\frac{\kappa}{4}}\left(S^{i}\right) \text { for all } t_{2}<t_{1} \text { and } t \geq 0 \text {. }
$$

Due to (7.10) and $\frac{\delta}{2}<\frac{\kappa}{4}$, we have

$$
\lambda\left(t_{2}-t, t_{2}, U_{\kappa}\left(S^{i}\right)\right) \subset U_{\frac{\kappa}{2}}\left(S^{i}\right) \text { for all } t_{2}<t_{1} \text { and } t \in[T, 2 T]
$$

Suppose now, there exist $\hat{t}>2 T$ and $\hat{t}_{2}<t_{1}$ with

$$
d\left(\lambda\left(\hat{t}_{2}-\hat{t}, \hat{t}_{2}, U_{\kappa}\left(S^{i}\right)\right) \mid S^{i}\right) \geq \frac{\kappa}{2} .
$$

We define

$$
s:=\inf \left\{t>2 T: d\left(\lambda\left(\hat{t}_{2}-t, \hat{t}_{2}, U_{\kappa}\left(S^{i}\right)\right) \mid S^{i}\right) \geq \frac{\kappa}{2}\right\}>2 T
$$

and set $t_{2}:=\hat{t}_{2}-s+T<t_{1}$. Consequently,

$$
\lambda\left(\hat{t}_{2}-s, \hat{t}_{2}, U_{\kappa}\left(S^{i}\right)\right)=\lambda(t_{2}-T, t_{2}, \underbrace{\lambda\left(t_{2}, \hat{t}_{2}, U_{\kappa}\left(S^{i}\right)\right)}_{\subset U_{\frac{\kappa}{2}}\left(S^{i}\right)}) \stackrel{(7.11)}{\in} U_{\frac{\kappa}{4}}\left(S^{i}\right)
$$

holds. This is a contradiction, i.e., for all $t_{2}<t_{1}$ and $t>T$, we have

$$
\lambda\left(t_{2}-t, t_{2}, U_{\kappa}\left(S^{i}\right)\right) \subset U_{\frac{\kappa}{2}}\left(S^{i}\right) .
$$

Since $\mu(t) \in U_{\frac{\kappa}{6}}\left(x_{0}\right)$ for all $t<t_{1}$, the relation

$$
\lambda\left(t_{2}-t, t_{2}, U_{\frac{5 \kappa}{6}}\left(\mathcal{A}_{\mu}^{\leftarrow}+\mu\left(t_{2}\right)\right)\right) \subset U_{\frac{2 \kappa}{3}}\left(\mathcal{A}_{\mu}^{\leftarrow}+\mu\left(t_{2}-t\right)\right) \quad \text { for all } t_{2}<t_{1} \text { and } t>T
$$

is fulfilled.

STEP 4. Existence of the past repeller.

Repeated usage of Step 3 implies

$$
\lim _{t \rightarrow-\infty} d\left(\lambda\left(t, \tau, U_{\frac{5 \eta}{6}}\left(\mathcal{A}_{\mu}^{\leftarrow}+\mu(\tau)\right)\right) \mid \mathcal{A}_{\mu}^{\leftarrow}+\mu(t)\right)=0 \quad \text { for all } \tau<t_{1}
$$


Because of Theorem 2.1.31 (i), there exist an $s<\tau$ and a past repeller $R \subset(-\infty, s) \times D$ with

$$
\mathcal{A}_{\mu}^{\leftarrow} \subset \liminf _{t \rightarrow-\infty}(R(t)-\mu(t)) \subset \limsup _{t \rightarrow-\infty}(R(t)-\mu(t)) \subset \operatorname{cls} \mathcal{A}_{\mu}^{\leftarrow}
$$

Since $\lim _{t \rightarrow-\infty} \mu(t)=x_{0}$, we have

$$
S^{i} \subset \liminf _{t \rightarrow-\infty} R(t) \subset \limsup _{t \rightarrow-\infty} R(t) \subset \operatorname{cls} S^{i} .
$$

This finishes the proof of this lemma.

7.1.6 Lemma (Attraction Areas of Future Attractive Solutions). We suppose that Hypothesis $\left(\mathrm{H}_{1}\right)$ is fulfilled and consider the nonautonomous differential equation

$$
\dot{x}=f(t, x)
$$

with a $C^{1}$-function $f:(0, \infty) \times D \rightarrow \mathbb{R}^{N}$ such that

$$
\lim _{t \rightarrow \infty} f(t, x)=g(x) \quad \text { uniformly for all } x \in D .
$$

Furthermore, for some $\tau>0$, let $\mu:(\tau, \infty) \rightarrow \mathbb{R}^{N}$ be a future attractive solution of (7.12) with $\lim _{t \rightarrow \infty} \mu(t)=x_{0}$. Then, we have

$$
\mathcal{A}_{\mu}=S^{i}-x_{0} .
$$

If, in addition, $S^{i}$ is bounded, then there exist $s>\tau$ and a future repeller $R \subset(s, \infty) \times D$ with

$$
S^{i} \subset \liminf _{t \rightarrow \infty} R(t) \subset \limsup _{t \rightarrow \infty} R(t) \subset \operatorname{cls} S^{i}
$$

PROOF. The proof of this lemma is quite similar to that of Lemma 7.1.5, but this is not clear a priori and there are important differences. In the following, the entire proof is therefore written down.

STEP 1. $\mathcal{A}_{\mu} \supset S^{i}-x_{0}$.

Since $\mu$ is future attractive, there exists a $\gamma>0$ such that for all $s>\tau$, we have

$$
\lim _{t \rightarrow \infty} d\left(\lambda\left(t, s, U_{\gamma}(\mu(s))\right) \mid \mu(t)\right)=0 .
$$

We choose $y \in S^{i}$ arbitrarily. Let $C$ be a neighborhood of $y$ such that there exists a $\delta>0$ with cls $U_{\delta}(C) \subset S^{i}$. Since $\lim _{t \rightarrow \infty} \mu(t)=x_{0}$, there exists a $t_{1}>\tau$ such that

$$
\mu(t) \in U_{\min }\left\{\frac{\gamma}{3}, \delta\right\}^{\left(x_{0}\right)} \text { for all } t \geq t_{1} .
$$

Due to the attractivity of $x_{0}$, there exists a $T>0$ such that

$$
d\left(\phi\left(T, U_{\delta}(C)\right) \mid\left\{x_{0}\right\}\right)<\frac{\gamma}{3} .
$$

Since it is possible to choose a compact and convex superset $K \subset D$ of $S^{i}$ ( $D$ is convex), Lemma 7.1.2 (i) implies that there exists a $t_{2}>t_{1}$ with

$$
\|\lambda(t+T, t, x)-\phi(T, x)\| \leq \frac{\gamma}{3} \quad \text { for all } t \geq t_{2} \text { and } x \in \underbrace{\mu(t)+C-x_{0}}_{\subset U_{\delta}(C)} .
$$


Hence, for all $t \geq t_{2}$ and $x \in \mu(t)+C-x_{0}$, we have

$$
\begin{aligned}
& \|\lambda(t+T, t, x)-\mu(t+T)\| \\
\leq & \|\lambda(t+T, t, x)-\phi(T, x)\|+\left\|\phi(T, x)-x_{0}\right\|+\left\|x_{0}-\mu(t+T)\right\| \\
< & \frac{\gamma}{3}+\frac{\gamma}{3}+\frac{\gamma}{3}=\gamma .
\end{aligned}
$$

Thus, for all $s \geq t_{2}$, we have the relation

$$
\begin{aligned}
& \lim _{t \rightarrow \infty} d\left(\lambda\left(t, s, \mu(s)+C-x_{0}\right) \mid\{\mu(s)\}\right) \\
= & \lim _{t \rightarrow \infty} d\left(\lambda(t, s+T, \underbrace{\lambda\left(s+T, t, \mu(s)+C-x_{0}\right)}_{\subset U_{\gamma}(\mu(s+T))}) \mid\{\mu(t)\}\right) \stackrel{(7.13)}{=} 0 .
\end{aligned}
$$

This implies $y-x_{0} \in \mathcal{A}_{\mu}$, and since $y \in S^{i}$ has been chosen arbitrarily, we have $\mathcal{A}_{\mu} \supset S^{i}-x_{0}$. STEP 2. $\mathcal{A}_{\mu} \subset S^{i}-x_{0}$.

We choose $y \in S^{o}$ and $\beta>0$ such that $U_{\beta}\left(x_{0}\right) \subset S^{i}$ and define $\delta:=d(y, S)>0$. Due to Lemma 7.1.3 (i), there exists a $T>0$ such that for all $x \in S^{o}$ with $d(x, S) \geq \min \left\{\frac{\delta}{2}, \frac{\eta}{3}\right\}$, there exists a $\hat{T} \in[0, T]$ with

$$
d(\phi(\hat{T}, x), S) \geq \eta \quad \text { and } \quad \phi(\hat{T}, x) \in S^{o}
$$

Moreover, there exists a $t_{1}>\tau$ with

$$
\mu(t) \in U_{\min }\left\{\frac{\delta}{2}, \frac{\eta}{3}, \frac{\beta}{4}\right\}\left(_{0}\right) \subset S^{i} \text { for all } t \geq t_{1} .
$$

Let $K$ be a compact and convex superset of $U_{\eta}(S)$. Then, due to Lemma 7.1.2 (i), there exists a $t_{2}>t_{1}$ such that for all $\tilde{T} \in[0, T]$ and $x \in K$ with $\phi(t, x) \in K$ for all $t \in[0, \tilde{T}]$, we have

$$
\|\lambda(\hat{t}+t, \hat{t}, x)-\phi(t, x)\| \leq \min \left\{\frac{\beta}{2}, \frac{\eta}{3}\right\} \quad \text { for all } \hat{t} \geq t_{2} \text { and } t \in[0, \tilde{T}]
$$

We argue negatively and suppose, there exists an $t_{3} \geq t_{2}$ such that the relation

$$
\lim _{t \rightarrow \infty}\left\|\lambda\left(t, t_{3}, y-x_{0}+\mu\left(t_{3}\right)\right)-\mu(t)\right\|=0
$$

holds. Therefore, since $\lim _{t \rightarrow \infty} \mu(t)=x_{0}$, there exists a $t_{4}>t_{3}$ with

$$
\lambda\left(t_{4}, t_{3}, y-x_{0}+\mu\left(t_{3}\right)\right) \in U_{\frac{\beta}{2}}\left(x_{0}\right) .
$$

We define

$$
\begin{array}{r}
s:=\max \left\{t \in\left[t_{3}, t_{4}\right]: d\left(\lambda\left(t, t_{3}, y-x_{0}+\mu\left(t_{3}\right)\right), S\right) \geq \min \left\{\frac{\eta}{2}, \frac{\delta}{2}\right\}\right. \\
\text { and } \left.\quad \lambda\left(t_{,} t_{3}, y-x_{0}+\mu\left(t_{3}\right)\right) \in S^{o}\right\} .
\end{array}
$$

This implies $d\left(\lambda\left(s, t_{3}, y-x_{0}+\mu\left(t_{3}\right)\right), S\right)=\min \left\{\frac{\eta}{2}, \frac{\delta}{2}\right\}$ and $\lambda\left(s, t_{3}, y-x_{0}+\mu\left(t_{3}\right)\right) \in S^{o}$. We distinguish two cases. 
CASE $1 . t_{4}-s \leq T$.

CASE 1.1. For all $t \in\left[0, t_{4}-s\right]$, we have $\phi\left(t, \lambda\left(s, t_{3}, y-x_{0}+\mu\left(t_{3}\right)\right)\right) \in K$.

Due to (7.15), we have

$$
\left\|\phi\left(t, \lambda\left(s, t_{3}, y-x_{0}+\mu\left(t_{3}\right)\right)\right)-\lambda\left(t+s, t_{3}, y-x_{0}+\mu\left(t_{3}\right)\right)\right\| \leq \frac{\beta}{2} \quad \text { for all } t \in\left[0, t_{4}-s\right] .
$$

Since $\phi\left(t, \lambda\left(s, t_{3}, y-x_{0}+\mu\left(t_{3}\right)\right)\right) \in S^{o}$ for all $t \in\left[0, t_{4}-s\right]$, this leads to

$$
\begin{aligned}
& \left\|\lambda\left(t_{4}, t_{3}, y-x_{0}+\mu\left(t_{3}\right)\right)-x_{0}\right\| \\
\geq & \left\|\phi\left(t_{4}-s, \lambda\left(s, t_{3}, y-x_{0}+\mu\left(t_{3}\right)\right)\right)-x_{0}\right\|- \\
& \left\|\lambda\left(t_{4}, t_{3}, y-x_{0}+\mu\left(t_{3}\right)\right)-\phi\left(t_{4}-s, \lambda\left(s, t_{3}, y-x_{0}+\mu\left(t_{3}\right)\right)\right)\right\| \\
\geq & \beta-\frac{\beta}{2}=\frac{\beta}{2} .
\end{aligned}
$$

This is a contradiction to (7.16).

CASE 1.2. There exists a $\tilde{t} \in\left[0, t_{4}-s\right]$ with $\phi\left(\tilde{t}, \lambda\left(s, t_{3}, y-x_{0}+\mu\left(t_{3}\right)\right)\right) \notin K$.

By defining

$$
\hat{s}:=\inf \left\{t \in\left[0, t_{4}-s\right]: \phi\left(t, \lambda\left(s, t_{3}, y-x_{0}+\mu\left(t_{3}\right)\right)\right) \notin K\right\}>0,
$$

we have $d\left(\phi\left(\hat{s}, \lambda\left(s, t_{3}, y-x_{0}+\mu\left(t_{3}\right)\right)\right), S\right) \geq \eta$. Due to (7.15), the relation

$$
\left\|\lambda\left(\hat{s}+s, t_{3}, y-x_{0}+\mu\left(t_{3}\right)\right)-\phi\left(\hat{s}, \lambda\left(s, t_{3}, y-x_{0}+\mu\left(t_{3}\right)\right)\right)\right\| \leq \frac{\eta}{3}
$$

holds. Hence, we have $d\left(\lambda\left(\hat{s}+s, t_{3}, y-x_{0}+\mu\left(t_{3}\right)\right), S\right) \geq \frac{2 \eta}{3}$ and $\lambda\left(\hat{s}+s, t_{3}, y-x_{0}+\mu\left(t_{3}\right)\right) \in S^{o}$. This is a contradiction to the definition of $s$.

CASE 2. $t_{4}-s>T$.

CASE 2.1. For all $t \in[0, T]$, we have $\phi\left(t, \lambda\left(s, t_{3}, y-x_{0}+\mu\left(t_{3}\right)\right)\right) \in K$.

Because of (7.14) and $d\left(\lambda\left(s, t_{3}, y-x_{0}+\mu\left(t_{3}\right)\right), S\right)=\min \left\{\frac{\delta}{2}, \frac{\eta}{2}\right\} \geq \min \left\{\frac{\delta}{2}, \frac{\eta}{3}\right\}$, there exists a $\hat{T} \in[0, T]$ with

$$
d\left(\phi\left(\hat{T}, \lambda\left(s, t_{3}, y-x_{0}+\mu\left(t_{3}\right)\right)\right), S\right) \geq \eta
$$

and (7.15) yields

$$
\left\|\phi\left(\hat{T}, \lambda\left(s, t_{3}, y-x_{0}+\mu\left(t_{3}\right)\right)\right)-\lambda\left(\hat{T}+s, t_{3}, y-x_{0}+\mu\left(t_{3}\right)\right)\right\| \leq \frac{\eta}{3} .
$$

Together, this implies

$$
d\left(\lambda\left(\hat{T}+s, t_{3}, y-x_{0}+\mu\left(t_{3}\right)\right), S\right) \geq \frac{2}{3} \eta \quad \text { and } \quad \lambda\left(\hat{T}+s, t_{3}, y-x_{0}+\mu\left(t_{3}\right)\right) \in S^{o} .
$$

This is a contradiction to the definition of $s$.

CASE 2.2. There exists a $\tilde{t} \in[0, T]$ with $\phi\left(\tilde{t}, \lambda\left(s, t_{3}, y-x_{0}+\mu\left(t_{3}\right)\right)\right) \notin K$.

This case is treated analogously to Case 1.2 (by writing $T$ instead of $t_{4}-s$ ).

Consequently, we have $y-x_{0} \notin \mathcal{A}_{\mu}$. This leads to the assertion, since $S=\partial S^{i}$, int $S=\emptyset$ and $\mathcal{A}_{\mu} \rightarrow$ is open.

STEP 3. For all $\kappa \leq \eta$, there exist $T>0$ and $t_{1}>0$ such that for all $t_{2}>t_{1}$ and $t>T$, we have

$$
\lambda\left(t_{2}, t_{2}+t, U_{\frac{5 \kappa}{6}}\left(\mathcal{A}_{\mu}+\mu\left(t_{2}+t\right)\right)\right) \subset U_{\frac{2 \kappa}{3}}\left(\mathcal{A}_{\mu}+\mu\left(t_{2}\right)\right) .
$$


We choose $\kappa \leq \eta$ arbitrarily. By applying Lemma 7.1.3 (ii), there exists a $\delta>0$ with

$$
\phi\left(-t, U_{\delta}\left(S^{i}\right)\right) \subset U_{\frac{\kappa}{4}}\left(S^{i}\right) \text { for all } t \geq 0 .
$$

Due to the repulsivity of $S$, there exists a $T>0$ with

$$
\phi\left(-t, U_{\kappa}\left(S^{i}\right)\right) \subset U_{\frac{\delta}{2}}\left(S^{i}\right) \text { for all } t>T .
$$

By choosing $K$ as a convex and compact superset of $\cup_{t \in[0, T]} \phi\left(-t, U_{\kappa}\left(S^{i}\right)\right)$, we can apply Lemma 7.1.1 (ii), and we therefore get a $t_{1}>\tau$ with

$$
\text { and } \begin{aligned}
\left\|\lambda\left(t_{2}, t_{2}+t, x\right)-\phi(-t, x)\right\| & \leq \frac{\delta}{2} & & \text { for all } x \in U_{\kappa}\left(S^{i}\right), t_{2}>t_{1} \text { and } t \in[0, T] \\
\left\|\mu(t)-x_{0}\right\| & \leq \frac{\kappa}{6} & & \text { for all } t>t_{1} .
\end{aligned}
$$

Thus,

$$
\lambda\left(t_{2}, t_{2}+T, U_{\kappa}\left(S^{i}\right)\right) \subset U_{\delta}\left(S^{i}\right) \subset U_{\frac{\kappa}{4}}\left(S^{i}\right) \text { for all } t_{2}>t_{1}
$$

is fulfilled. Because of (7.17), this leads to

$$
\phi\left(-t, \lambda\left(t_{2}-T, t_{2}, U_{\kappa}\left(S^{i}\right)\right)\right) \subset U_{\frac{\kappa}{4}}\left(S^{i}\right) \text { for all } t_{2}>t_{1} \text { and } t \geq 0 .
$$

Due to (7.18) and $\frac{\delta}{2}<\frac{\kappa}{4}$, we have

$$
\lambda\left(t_{2}, t_{2}+t, U_{\kappa}\left(S^{i}\right)\right) \subset U_{\frac{\kappa}{2}}\left(S^{i}\right) \text { for all } t_{2}>t_{1} \text { and } t \in[T, 2 T] .
$$

Suppose now that there exist $\hat{t}>2 T$ and $\hat{t}_{2}<t_{1}$ with

$$
d\left(\lambda\left(\hat{t}_{2}, \hat{t}_{2}+\hat{t}, U_{\kappa}\left(S^{i}\right)\right) \mid S^{i}\right) \geq \frac{\kappa}{2} .
$$

We define

$$
s:=\inf \left\{t>2 T: d\left(\lambda\left(\hat{t}_{2}, \hat{t}_{2}+t, U_{\kappa}\left(S^{i}\right)\right) \mid S^{i}\right) \geq \frac{\kappa}{2}\right\}>2 T
$$

and set $t_{2}:=\hat{t}_{2}+s-T>t_{1}$. Consequently,

$$
\lambda\left(\hat{t}_{2}, \hat{t}_{2}+s, U_{\kappa}\left(S^{i}\right)\right)=\lambda(\hat{t}_{2}, t_{2}, \underbrace{\lambda\left(t_{2}, \hat{t}_{2}+s, U_{\kappa}\left(S^{i}\right)\right)}_{\substack{(7.19) \\ \subset} \frac{\kappa}{2}\left(S^{i}\right)}) \in U_{\frac{\kappa}{4}}\left(S^{i}\right)
$$

holds. This is a contradiction, i.e., for all $t_{2}<t_{1}$ and $t>T$, we have

$$
\lambda\left(t_{2}, t_{2}+t, U_{\kappa}\left(S^{i}\right)\right) \subset U_{\frac{\kappa}{2}}\left(S^{i}\right) .
$$

Since $\mu(t) \in U_{\frac{\kappa}{6}}\left(x_{0}\right)$ for all $t>t_{1}$, the relation

$$
\lambda\left(t_{2}, t_{2}+t, U_{\frac{5 \kappa}{6}}\left(S^{i}+\mu\left(t_{2}+t\right)\right)\right) \subset U_{\frac{2 \kappa}{3}}\left(S^{i}+\mu\left(t_{2}\right)\right) \quad \text { for all } t_{2}>t_{1} \text { and } t>T
$$

is fulfilled.

STEP 4. Existence of the future repeller.

Repeated usage of Step 3 implies that for all $\varepsilon>0$, there exists an $s>0$ such that for all $\tau \geq s$, there exists a $T>0$ with

$$
\lambda\left(\tau, \tau+t, U_{\frac{\eta}{2}}\left(\mathcal{A}_{\mu}+\mu(\tau+t)\right)\right) \subset U_{\varepsilon}\left(\mathcal{A}_{\mu}+\mu(\tau)\right) \quad \text { for all } t \geq T .
$$


Because of Theorem 2.1.31 (iv), there exists an $s<\tau$ and a future repeller $R \subset(s, \infty) \times D$ with

$$
\mathcal{A}_{\mu} \overrightarrow{\liminf _{t \rightarrow \infty}}(R(t)-\mu(t)) \subset \limsup _{t \rightarrow \infty}(R(t)-\mu(t)) \subset \operatorname{cls} \mathcal{A}_{\mu} .
$$

Since $\lim _{t \rightarrow \infty} \mu(t)=x_{0}$, we have

$$
S^{i} \subset \liminf _{t \rightarrow \infty} R(t) \subset \limsup _{t \rightarrow \infty} R(t) \subset \operatorname{cls} S^{i} .
$$

This finishes the proof of this lemma.

Please note that similar lemmata can be derived for the determination of past (future, respectively) repulsion areas of past (future, respectively) repulsive solutions.

\subsection{BIFURCATIONS IN DIMENSION ONE}

In this section, one-dimensional differential equations are studied which exhibit pitchfork, transcritical or saddle node bifurcations. It is shown that under special assumptions, this bifurcation behavior is transferred to asymptotically autonomous systems.

Let $-\infty \leq x_{-}<x_{+} \leq \infty$ and $\alpha_{0}<\alpha_{1}$, and consider an autonomous differential equation

$$
\dot{x}=g(x, \alpha)
$$

depending on a parameter $\alpha$ with a $C^{1}$-function $g:\left(x_{-}, x_{+}\right) \times\left(\alpha_{0}, \alpha_{1}\right] \rightarrow \mathbb{R}$. We assume that there exists an $x_{0} \in\left(x_{-}, x_{+}\right)$with

$$
g\left(x_{0}, \alpha\right)=0 \quad \text { and } \quad D_{1} g\left(x_{0}, \alpha\right) \neq 0 \quad \text { for all } \alpha \in\left(\alpha_{0}, \alpha_{1}\right] .
$$

In the next four lemmata, conditions for the existence of nonautonomous counterparts for the equilibrium $x_{0}$ are studied. In a first instance, we restrict the parameter area to compact subintervals of $\left(\alpha_{0}, \alpha_{1}\right]$.

7.2.1 Lemma (Existence of Past Attractive Solutions). Let $\alpha_{-} \leq \alpha_{+}$be in $\left(\alpha_{0}, \alpha_{1}\right]$, and consider the nonautonomous differential equation

$$
\dot{x}=f(t, x, \alpha)
$$

depending on a parameter $\alpha$ with a $C^{1}$-function $f:(-\infty, 0) \times\left(x_{-}, x_{+}\right) \times\left[\alpha_{-}, \alpha_{+}\right] \rightarrow \mathbb{R}$. We assume that

$$
\lim _{t \rightarrow-\infty} f(t, x, \alpha)=g(x, \alpha) \quad \text { and } \quad \lim _{t \rightarrow-\infty} D_{2} f(t, x, \alpha)=D_{1} g(x, \alpha)
$$

hold uniformly for all $x \in\left(x_{-}, x_{+}\right)$and $\alpha \in\left[\alpha_{-}, \alpha_{+}\right]$. Furthermore, we suppose that

$$
D_{1} g\left(x_{0}, \alpha\right)<0 \quad \text { for all } \alpha \in\left[\alpha_{-}, \alpha_{+}\right] \text {. }
$$

Then, there exist a $\tau<0$ and a continuous function $\mu:(-\infty, \tau] \times\left[\alpha_{-}, \alpha_{+}\right] \rightarrow \mathbb{R}$ such that $\mu(\cdot, \alpha)$ is the uniquely determined past attractive solution of $(7.21)_{\alpha}$ which fulfills

$$
\lim _{t \rightarrow-\infty} \mu(t, \alpha)=x_{0} .
$$


In addition, for fixed $\alpha \in\left[\alpha_{-}, \alpha_{+}\right]$, the following statements are fulfilled:

(i) In case there exist $x_{0}^{-}<x_{0}$ and $x_{0}^{+}>x_{0}$ with

$$
g\left(x_{0}^{-}, \alpha\right)=g\left(x_{0}^{+}, \alpha\right)=0, \quad D_{1} g\left(x_{0}^{-}, \alpha\right)>0 \quad \text { and } \quad D_{1} g\left(x_{0}^{+}, \alpha\right)>0,
$$

and $g(x, \alpha) \neq 0$ for all $x \in\left(x_{0}^{-}, x_{0}\right) \cup\left(x_{0}, x_{0}^{+}\right)$, we have

$$
\mathcal{A}_{\mu(\cdot, \alpha)}^{\leftarrow}=\left(x_{0}^{-}-x_{0}, x_{0}^{+}-x_{0}\right)
$$

Furthermore, there exists a past repeller $R(\alpha)$ of $(7.21)_{\alpha}$ with

$$
\left(x_{0}^{-}, x_{0}^{+}\right) \subset \liminf _{t \rightarrow-\infty} R(\alpha, t) \subset \limsup _{t \rightarrow-\infty} R(\alpha, t) \subset\left[x_{0}^{-}, x_{0}^{+}\right] .
$$

(ii) In case there exists a $x_{0}^{-}<x_{0}$ with

$$
g\left(x_{0}^{-}, \alpha\right)=0 \quad \text { and } \quad D_{1} g\left(x_{0}^{-}, \alpha\right)>0,
$$

and $g(x, \alpha) \neq 0$ for all $x \in\left(x_{0}^{-}, x_{0}\right) \cup\left(x_{0}, x_{+}\right)$, we have

$$
\mathcal{A}_{\mu(\cdot, \alpha)}^{\leftarrow}=\left(x_{0}^{-}-x_{0}, x_{+}-x_{0}\right)
$$

(iii) In case there exists a $x_{0}^{+}>x_{0}$ with

$$
g\left(x_{0}^{+}, \alpha\right)=0 \quad \text { and } \quad D_{1} g\left(x_{0}^{+}, \alpha\right)>0,
$$

and $g(x, \alpha) \neq 0$ for all $x \in\left(x_{-}, x_{0}\right) \cup\left(x_{0}, x_{0}^{+}\right)$, we have

$$
\mathcal{A}_{\mu(\cdot, \alpha)}^{\leftarrow}=\left(x_{-}-x_{0}, x_{0}^{+}-x_{0}\right)
$$

7.2.2 REMARK. The statement (i) of above lemma corresponds to the autonomous pitchfork bifurcation, where after the bifurcation, there are three equilibria, and (ii) and (iii) describe the situation after a transcritical or saddle node bifurcation.

PROOF OF LEMMA 7.2.1. The proof is divided into three steps.

STEP 1. There exist a $\tau<0$ and a continuous function $\mu:(-\infty, \tau] \times\left[\alpha_{-}, \alpha_{+}\right] \rightarrow \mathbb{R}$ such that $\mu(\cdot, \alpha)$ is the uniquely determined past attractive solution of $(7.21)_{\alpha}$ which fulfills $\lim _{t \rightarrow-\infty} \mu(t, \alpha)=x_{0}$.

Due to the hypotheses (please note that $\left[\alpha_{-}, \alpha_{+}\right]$is compact and $g$ is uniformly continuous on compact sets), there exist $\beta>0, \gamma<0$ and $\tau<0$ with

$$
f\left(t, x_{0}-\beta, \alpha\right)>0, f\left(t, x_{0}+\beta, \alpha\right)<0 \quad \text { and } \quad D_{2} f(t, x, \alpha) \leq \gamma
$$

for all $x \in U_{2 \beta}\left(x_{0}\right), t \leq \tau$ and $\alpha \in\left[\alpha_{-}, \alpha_{+}\right]$. We fix an $\alpha \in\left[\alpha_{-}, \alpha_{+}\right]$for the rest of this step. The sets

$$
M_{1}:=\left\{x \in \operatorname{cls} U_{\beta}\left(x_{0}\right): \text { There exists a } t<\tau \text { with } \lambda(t, \tau, x, \alpha)<x_{0}-\beta\right\}
$$

and

$$
M_{2}:=\left\{x \in \operatorname{cls} U_{\beta}\left(x_{0}\right): \text { There exists a } t<\tau \text { with } \lambda(t, \tau, x, \alpha)>x_{0}+\beta\right\}
$$


are obviously nonempty and due to the continuity of the general solution (cf. Proposition A.1.3) relatively open in cls $U_{\beta}\left(x_{0}\right)$. Hence, $M_{1} \cup M_{2} \subsetneq \operatorname{cls} U_{\beta}\left(x_{0}\right)$. Therefore, there exists a $y \in U_{\beta}\left(x_{0}\right)$ such that $\mu(t, \alpha):=\lambda(t, \tau, y, \alpha) \in U_{\beta}\left(x_{0}\right)$ for all $t \leq \tau$. To show that this solution is past attractive, we study the differential equation of the perturbed motion

$$
\dot{x}=h(t, x, \alpha):=f(t, x+\mu(t, \alpha), \alpha)-f(t, \mu(t, \alpha), \alpha),
$$

whose general solution will be denoted by $\tilde{\lambda}$. Due to the mean value theorem (see, e.g., LANG [101, Theorem 4.2, p. 341]), we have

$$
h(t, x, \alpha)=x \int_{0}^{1} D_{2} h(t, \theta x, \alpha) d \theta=x \int_{0}^{1} D_{2} f(t, \theta x+\mu(t, \alpha), \alpha) d \theta .
$$

This implies

$$
\begin{array}{ll}
h(t, x, \alpha) \geq \gamma x & \text { for all } t \leq \tau \text { and } x \in(-\beta, 0) \\
\text { and } \quad h(t, x, \alpha) \leq \gamma x & \text { for all } t \leq \tau \text { and } x \in(0, \beta) .
\end{array}
$$

We therefore obtain

$$
\lim _{t \rightarrow-\infty} d\left(\tilde{\lambda}\left(\tau, t, U_{\frac{\beta}{2}}(0), \alpha\right) \mid\{0\}\right)=0,
$$

and consequently,

$$
\lim _{t \rightarrow-\infty} d\left(\lambda\left(\tau, t, U_{\frac{\beta}{2}}(\mu(t, \alpha)), \alpha\right) \mid\{\mu(\tau, \alpha)\}\right)=0
$$

holds (cf. Proposition A.1.7). Thus, the solution $\mu(\cdot, \alpha)$ is past attractive. Moreover, the limit relation $\lim _{t \rightarrow-\infty} \mu(t, \alpha)=x_{0}$ is obviously fulfilled. The uniqueness of $\mu(\cdot, \alpha)$ follows directly from Proposition 2.1.27 (i).

STEP $2 . \mu$ is continuous.

First, we consider the function $d:\left[\alpha_{-}, \alpha_{+}\right] \rightarrow\left(x_{-}, x_{+}\right)$, defined by

$$
d(\alpha):=\mu(\tau, \alpha) \text { for all } \alpha \in\left[\alpha_{-}, \alpha_{+}\right] .
$$

Suppose, there exist a $\tilde{\alpha} \in\left[\alpha_{-}, \alpha_{+}\right]$and a sequence $\left\{\tilde{\alpha}_{n}\right\}_{n \in \mathbb{N}}$ with $\lim _{n \rightarrow \infty} \tilde{\alpha}_{n}=\tilde{\alpha}$ such that $\left\{d\left(\tilde{\alpha}_{n}\right)\right\}_{n \in \mathbb{N}}$ does not converge to $d(\tilde{\alpha})$. Since this sequence is bounded, we assume w.l.o.g. that it is convergent with limit $\tilde{x} \in \operatorname{cls} U_{\beta}\left(x_{0}\right), \tilde{x} \neq d(\tilde{\alpha})$. Due to Step 1 , there exists a $\tilde{t}<\tau$ with $\lambda(\tilde{t}, \tau, \tilde{x}, \tilde{\alpha}) \notin \operatorname{cls} U_{\beta}\left(x_{0}\right)$. The continuity of the general solution implies the existence of a neighborhood $V$ of $(\tilde{x}, \tilde{\alpha})$ such that

$$
\lambda(\tilde{t}, \tau, x, \alpha) \notin \operatorname{cls} U_{\beta}\left(x_{0}\right) \quad \text { for all }(x, \alpha) \in V .
$$

In particular, there exists an $n \in \mathbb{N}$ with $\left(d\left(\tilde{\alpha}_{n}\right), \tilde{\alpha}_{n}\right) \in V$. This implies

$$
\mu\left(\tilde{t}, \tilde{\alpha}_{n}\right)=\lambda\left(\tilde{t}, \tau, \mu\left(\tau, \tilde{\alpha}_{n}\right), \tilde{\alpha}_{n}\right)=\lambda\left(\tilde{t}, \tau, d\left(\tilde{\alpha}_{n}\right), \tilde{\alpha}_{n}\right) \notin \operatorname{cls} U_{\beta}\left(x_{0}\right) .
$$

This is a contradiction, and therefore, the function $d$ is continuous. To prove the continuity of $\mu$, we choose a sequence $\left\{\left(\hat{t}_{n}, \hat{\alpha}_{n}\right)\right\}_{n \in \mathbb{N}}$ in $(-\infty, \tau] \times\left[\alpha_{-}, \alpha_{+}\right]$with $\lim _{n \rightarrow \infty}\left(\hat{t}_{n}, \hat{\alpha}_{n}\right)=(\hat{t}, \hat{\alpha})$. The continuity of $\mu$ follows from

$$
\begin{aligned}
\lim _{n \rightarrow \infty} \mu\left(\hat{t}_{n}, \hat{\alpha}_{n}\right) & =\lim _{n \rightarrow \infty} \lambda\left(\hat{t}_{n}, \tau, \mu\left(\tau, \hat{\alpha}_{n}\right), \hat{\alpha}_{n}\right) \\
& =\lim _{n \rightarrow \infty} \lambda\left(\hat{t}_{n}, \tau, d\left(\hat{\alpha}_{n}\right), \hat{\alpha}_{n}\right) \\
& =\lambda(\hat{t}, \tau, d(\hat{\alpha}), \hat{\alpha})=\mu(\hat{t}, \hat{\alpha}) .
\end{aligned}
$$


STEP 3. The statements (i), (ii) and (iii) are fulfilled.

The asserted relations for $\mathcal{A}_{\mu(\cdot, \alpha)}^{\leftarrow}$ and the existence of a past repeller follow directly from Lemma 7.1.5 if we define the repulsive set $S$ as $\left\{x_{0}^{-}, x_{0}^{+}\right\}$in case (i), $\left\{x_{0}^{-}\right\}$in case (ii) or $\left\{x_{0}^{+}\right\}$in case (iii), respectively (cf. also Figure 7.1).

7.2.3 Lemma (Existence of Future Attractive Solutions). Let $\alpha_{-} \leq \alpha_{+}$be in $\left(\alpha_{0}, \alpha_{1}\right]$, and consider the nonautonomous differential equation

$$
\dot{x}=f(t, x, \alpha)
$$

depending on a parameter $\alpha$ with a $C^{1}$-function $f:(0, \infty) \times\left(x_{-}, x_{+}\right) \times\left[\alpha_{-}, \alpha_{+}\right] \rightarrow \mathbb{R}$. We assume that

$$
\lim _{t \rightarrow \infty} f(t, x, \alpha)=g(x, \alpha) \text { and } \lim _{t \rightarrow \infty} D_{2} f(t, x, \alpha)=D_{1} g(x, \alpha)
$$

hold uniformly for all $x \in\left(x_{-}, x_{+}\right)$and $\alpha \in\left[\alpha_{-}, \alpha_{+}\right]$. Furthermore, we suppose that

$$
D_{1} g\left(x_{0}, \alpha\right)<0 \quad \text { for all } \alpha \in\left[\alpha_{-}, \alpha_{+}\right] \text {. }
$$

Then, there exist $\tau>0$ and $\beta>0$ such that every solution $\lambda(\cdot, \tau, x, \alpha)$ for $x \in U_{\beta}\left(x_{0}\right)$ and $\alpha \in\left[\alpha_{-}, \alpha_{+}\right]$is future attractive with

$$
\lim _{t \rightarrow \infty} \lambda(t, \tau, x, \alpha)=x_{0}
$$

Let $v:[\tau, \infty) \rightarrow \mathbb{R}$ be such a solution of $(7.22)_{\alpha}$ for fixed $\alpha \in\left[\alpha_{-}, \alpha_{+}\right]$. Then, the following statements are fulfilled:

(i) In case there exist $x_{0}^{-}<x_{0}$ and $x_{0}^{+}>x_{0}$ with

$$
g\left(x_{0}^{-}, \alpha\right)=g\left(x_{0}^{+}, \alpha\right)=0, \quad D_{1} g\left(x_{0}^{-}, \alpha\right)>0 \quad \text { and } \quad D_{1} g\left(x_{0}^{+}, \alpha\right)>0,
$$

and $g(x, \alpha) \neq 0$ for all $x \in\left(x_{0}^{-}, x_{0}\right) \cup\left(x_{0}, x_{0}^{+}\right)$, we have

$$
\mathcal{A}_{v} \rightarrow\left(x_{0}^{-}-x_{0}, x_{0}^{+}-x_{0}\right) .
$$

Furthermore, there exists a future repeller $R(\alpha)$ of $(7.22)_{\alpha}$ with

$$
\left(x_{0}^{-}, x_{0}^{+}\right) \subset \liminf _{t \rightarrow \infty} R(\alpha, t) \subset \limsup _{t \rightarrow \infty} R(\alpha, t) \subset\left[x_{0}^{-}, x_{0}^{+}\right] .
$$

(ii) In case there exists a $x_{0}^{-}<x_{0}$ with

$$
g\left(x_{0}^{-}, \alpha\right)=0 \quad \text { and } \quad D_{1} g\left(x_{0}^{-}, \alpha\right)>0,
$$

and $g(x, \alpha) \neq 0$ for all $x \in\left(x_{0}^{-}, x_{0}\right) \cup\left(x_{0}, x_{+}\right)$, we have

$$
\mathcal{A}_{v}^{\rightarrow}=\left(x_{0}^{-}-x_{0}, x_{+}-x_{0}\right) \text {. }
$$

(iii) In case there exists a $x_{0}^{+}>x_{0}$ with 


$$
g\left(x_{0}^{+}, \alpha\right)=0 \quad \text { and } D_{1} g\left(x_{0}^{+}, \alpha\right)>0,
$$

and $g(x, \alpha) \neq 0$ for all $x \in\left(x_{-}, x_{0}\right) \cup\left(x_{0}, x_{0}^{+}\right)$, we have

$$
\mathcal{A}_{v} \rightarrow=\left(x_{-}-x_{0}, x_{0}^{+}-x_{0}\right) \text {. }
$$

PROOF. Due to the hypotheses (please note that $\left[\alpha_{-}, \alpha_{+}\right]$is compact and $g$ is uniformly continuous on compact sets), there exist $\beta>0, \gamma<0$ and $\tau>0$ with

$$
f\left(t, x_{0}-\beta, \alpha\right)>0, f\left(t, x_{0}+\beta, \alpha\right)<0 \quad \text { and } \quad D_{2} f(t, x, \alpha) \leq \gamma
$$

for all $x \in U_{2 \beta}\left(x_{0}\right), t \geq \tau$ and $\alpha \in\left[\alpha_{-}, \alpha_{+}\right]$. We fix $\hat{x} \in U_{\beta}\left(x_{0}\right)$ and $\alpha \in\left[\alpha_{-}, \alpha_{+}\right]$and consider for the rest of the proof in particular the solution $v(\cdot):=\lambda(\cdot, \tau, \hat{x}, \alpha)$ of $(7.22)_{\alpha}$ on the interval $[\tau, \infty)$. Obviously, the relation $\lim _{t \rightarrow \infty} v(t)=x_{0}$ holds. To show that this solution is future attractive, we study the differential equation of the perturbed motion

$$
\dot{x}=h(t, x, \alpha):=f(t, x+v(t), \alpha)-f(t, v(t), \alpha),
$$

whose general solution will be denoted by $\tilde{\lambda}$. Due to the mean value theorem, we have

$$
h(t, x, \alpha)=x \int_{0}^{1} D_{2} h(t, \theta x, \alpha) d \theta=x \int_{0}^{1} D_{2} f(t, \theta x+v(t), \alpha) d \theta .
$$

This implies

$$
\begin{array}{lll} 
& h(t, x, \alpha) \geq \gamma x & \text { for all } t \geq \tau \text { and } x \in(-\beta, 0) \\
\text { and } & h(t, x, \alpha) \leq \gamma x & \text { for all } t \geq \tau \text { and } x \in(0, \beta) .
\end{array}
$$

We therefore obtain

$$
\lim _{t \rightarrow \infty} d\left(\tilde{\lambda}\left(t, s, U_{\frac{\beta}{2}}(0), \alpha\right) \mid\{0\}\right)=0 \quad \text { for all } s \geq \tau,
$$

and consequently,

$$
\lim _{t \rightarrow \infty} d\left(\lambda\left(t, s, U_{\frac{\beta}{2}}(v(s)), \alpha\right) \mid\{v(t)\}\right)=0 \quad \text { for all } s \geq \tau
$$

holds (cf. Proposition A.1.7). Thus, the solution $v$ is future attractive. The asserted relations for $\mathcal{A}_{v}$ and the existence of a future repeller follow directly from Lemma 7.1.6 if we define the repulsive set $S$ as $\left\{x_{0}^{-}, x_{0}^{+}\right\}$in case (i), $\left\{x_{0}^{-}\right\}$in case (ii) or $\left\{x_{0}^{+}\right\}$in case (iii), respectively (cf. also Figure 7.1).

Under the assumption $D_{1} g\left(x_{0}, \alpha\right)>0$ for all $\alpha \in\left[\alpha_{-}, \alpha_{+}\right]$, analogous statements are obtained for past (future, respectively) repulsive solutions.

7.2.4 Lemma (Existence of Past Repulsive Solutions). Let $\alpha_{-} \leq \alpha_{+}$be in $\left(\alpha_{0}, \alpha_{1}\right]$, and consider the nonautonomous differential equation

$$
\dot{x}=f(t, x, \alpha)
$$

depending on a parameter $\alpha$ with a $C^{1}$-function $f:(-\infty, 0) \times\left(x_{-}, x_{+}\right) \times\left[\alpha_{-}, \alpha_{+}\right] \rightarrow \mathbb{R}$. We assume that 


$$
\lim _{t \rightarrow-\infty} f(t, x, \alpha)=g(x, \alpha) \text { and } \lim _{t \rightarrow-\infty} D_{2} f(t, x, \alpha)=D_{1} g(x, \alpha)
$$

hold uniformly for all $x \in\left(x_{-}, x_{+}\right)$and $\alpha \in\left[\alpha_{-}, \alpha_{+}\right]$. Furthermore, we suppose that

$$
D_{1} g\left(x_{0}, \alpha\right)>0 \text { for all } \alpha \in\left[\alpha_{-}, \alpha_{+}\right] \text {. }
$$

Then, there exist $\tau<0$ and $\beta>0$ such that every solution $\lambda(\cdot, \tau, x, \alpha)$ for $x \in U_{\beta}\left(x_{0}\right)$ and $\alpha \in\left[\alpha_{-}, \alpha_{+}\right]$is past repulsive with

$$
\lim _{t \rightarrow-\infty} \lambda(t, \tau, x, \alpha)=x_{0}
$$

Let $v:(-\infty, \tau] \rightarrow \mathbb{R}$ be such a solution of $(7.23)_{\alpha}$ for fixed $\alpha \in\left[\alpha_{-}, \alpha_{+}\right]$. Then, the following statements are fulfilled:

(i) In case there exist $x_{0}^{-}<x_{0}$ and $x_{0}^{+}>x_{0}$ with

$$
g\left(x_{0}^{-}, \alpha\right)=g\left(x_{0}^{+}, \alpha\right)=0, \quad D_{1} g\left(x_{0}^{-}, \alpha\right)<0 \quad \text { and } \quad D_{1} g\left(x_{0}^{+}, \alpha\right)<0,
$$

and $g(x, \alpha) \neq 0$ for all $x \in\left(x_{0}^{-}, x_{0}\right) \cup\left(x_{0}, x_{0}^{+}\right)$, we have

$$
\mathcal{R}_{v}^{\leftarrow}=\left(x_{0}^{-}-x_{0}, x_{0}^{+}-x_{0}\right) .
$$

Furthermore, there exists a past attractor $A(\alpha)$ of $(7.23)_{\alpha}$ with

$$
\left(x_{0}^{-}, x_{0}^{+}\right) \subset \liminf _{t \rightarrow-\infty} A(\alpha, t) \subset \limsup _{t \rightarrow-\infty} A(\alpha, t) \subset\left[x_{0}^{-}, x_{0}^{+}\right] .
$$

(ii) In case there exists a $x_{0}^{-}<x_{0}$ with

$$
g\left(x_{0}^{-}, \alpha\right)=0 \quad \text { and } \quad D_{1} g\left(x_{0}^{-}, \alpha\right)<0,
$$

and $g(x, \alpha) \neq 0$ for all $x \in\left(x_{0}^{-}, x_{0}\right) \cup\left(x_{0}, x_{+}\right)$, we have

$$
\mathcal{R}_{v}^{\leftarrow}=\left(x_{0}^{-}-x_{0}, x_{+}-x_{0}\right)
$$

(iii) In case there exists a $x_{0}^{+}>x_{0}$ with

$$
g\left(x_{0}^{+}, \alpha\right)=0 \quad \text { and } \quad D_{1} g\left(x_{0}^{+}, \alpha\right)<0,
$$

and $g(x, \alpha) \neq 0$ for all $x \in\left(x_{-}, x_{0}\right) \cup\left(x_{0}, x_{0}^{+}\right)$, we have

$$
\mathcal{R}_{v}^{\leftarrow}=\left(x_{-}-x_{0}, x_{0}^{+}-x_{0}\right)
$$

PROOF. The assertions follow from Proposition 2.1.22 and Lemma 7.2.3.

7.2.5 Lemma (Existence of Future Repulsive Solutions). Let $\alpha_{-} \leq \alpha_{+}$be in $\left(\alpha_{0}, \alpha_{1}\right]$, and consider the nonautonomous differential equation 


$$
\dot{x}=f(t, x, \alpha)
$$

depending on a parameter $\alpha$ with a $C^{1}$-function $f:(0, \infty) \times\left(x_{-}, x_{+}\right) \times\left[\alpha_{-}, \alpha_{+}\right] \rightarrow \mathbb{R}$. We assume that

$$
\lim _{t \rightarrow \infty} f(t, x, \alpha)=g(x, \alpha) \text { and } \lim _{t \rightarrow \infty} D_{2} f(t, x, \alpha)=D_{1} g(x, \alpha)
$$

hold uniformly for all $x \in\left(x_{-}, x_{+}\right)$and $\alpha \in\left[\alpha_{-}, \alpha_{+}\right]$. Furthermore, we suppose that

$$
D_{1} g\left(x_{0}, \alpha\right)>0 \quad \text { for all } \alpha \in\left[\alpha_{-}, \alpha_{+}\right] \text {. }
$$

Then, there exist a $\tau>0$ and a continuous function $\mu:[\tau, \infty) \times\left[\alpha_{-}, \alpha_{+}\right] \rightarrow \mathbb{R}$ such that $\mu(\cdot, \alpha)$ is the uniquely determined future repulsive solution of $(7.24)_{\alpha}$ which fulfills

$$
\lim _{t \rightarrow \infty} \mu(t, \alpha)=x_{0}
$$

In addition, for fixed $\alpha \in\left[\alpha_{-}, \alpha_{+}\right]$, the following statements are fulfilled:

(i) In case there exist $x_{0}^{-}<x_{0}$ and $x_{0}^{+}>x_{0}$ with

$$
g\left(x_{0}^{-}, \alpha\right)=g\left(x_{0}^{+}, \alpha\right)=0, \quad D_{1} g\left(x_{0}^{-}, \alpha\right)<0 \quad \text { and } \quad D_{1} g\left(x_{0}^{+}, \alpha\right)<0,
$$

and $g(x, \alpha) \neq 0$ for all $x \in\left(x_{0}^{-}, x_{0}\right) \cup\left(x_{0}, x_{0}^{+}\right)$, we have

$$
\mathcal{R}_{\mu(\cdot, \alpha)}=\left(x_{0}^{-}-x_{0}, x_{0}^{+}-x_{0}\right) .
$$

Furthermore, there exists a future attractor $A(\alpha)$ of $(7.24)_{\alpha}$ with

$$
\left(x_{0}^{-}, x_{0}^{+}\right) \subset \liminf _{t \rightarrow \infty} A(\alpha, t) \subset \limsup _{t \rightarrow \infty} A(\alpha, t) \subset\left[x_{0}^{-}, x_{0}^{+}\right] .
$$

(ii) In case there exists a $x_{0}^{-}<x_{0}$ with

$$
g\left(x_{0}^{-}, \alpha\right)=0 \quad \text { and } \quad D_{1} g\left(x_{0}^{-}, \alpha\right)<0,
$$

and $g(x, \alpha) \neq 0$ for all $x \in\left(x_{0}^{-}, x_{0}\right) \cup\left(x_{0}, x_{+}\right)$, we have

$$
\mathcal{R}_{\mu(\cdot, \alpha)}=\left(x_{0}^{-}-x_{0}, x_{+}-x_{0}\right) \text {. }
$$

(iii) In case there exists a $x_{0}^{+}>x_{0}$ with

$$
g\left(x_{0}^{+}, \alpha\right)=0 \quad \text { and } \quad D_{1} g\left(x_{0}^{+}, \alpha\right)<0,
$$

and $g(x, \alpha) \neq 0$ for all $x \in\left(x_{-}, x_{0}\right) \cup\left(x_{0}, x_{0}^{+}\right)$, we have

$$
\mathcal{R}_{\mu(\cdot, \alpha)}^{\rightarrow}=\left(x_{-}-x_{0}, x_{0}^{+}-x_{0}\right) .
$$

PROOF. The assertions follow from Proposition 2.1.22 and Lemma 7.2.1.

In the following, we observe that pitchfork bifurcations of $(7.20)_{\alpha}$ give rise to total nonau- 
tonomous bifurcations. Transcritical and saddle node bifurcations, however, lead to partial nonautonomous bifurcations.

First, the attention is restricted to the situation that the autonomous differential equation (7.20) $\alpha$ admits a supercritical pitchfork bifurcation at $\left(x_{0}, \alpha_{0}\right)$. More precisely, there exist a monotone increasing continuous function $h_{1}:\left(\alpha_{0}, \alpha_{1}\right] \rightarrow\left(x_{-}, x_{+}\right)$and a monotone decreasing continuous function $h_{2}:\left(\alpha_{0}, \alpha_{1}\right] \rightarrow\left(x_{-}, x_{+}\right)$such that for all $\alpha \in\left(\alpha_{0}, \alpha_{1}\right]$, we have

$$
\begin{gathered}
h_{1}(\alpha)<x_{0}<h_{2}(\alpha), \\
g\left(h_{1}(\alpha), \alpha\right)=g\left(x_{0}, \alpha\right)=g\left(h_{2}(\alpha), \alpha\right)=0, \\
D_{1} g\left(h_{1}(\alpha), \alpha\right) \neq 0, D_{1} g\left(x_{0}, \alpha\right) \neq 0, D_{1} g\left(h_{2}(\alpha), \alpha\right) \neq 0 .
\end{gathered}
$$

Moreover, for all $\alpha \in\left(\alpha_{0}, \alpha_{1}\right]$ and $x \in\left(h_{1}(\alpha), x_{0}\right) \cup\left(x_{0}, h_{2}(\alpha)\right), g(x, \alpha) \neq 0$ is satisfied, and we have $\lim _{\alpha \rightarrow \alpha_{0}} h_{1}(\alpha)=\lim _{\alpha \rightarrow \alpha_{0}} h_{2}(\alpha)=x_{0}$.

7.2.6 Theorem (Total Past Bifurcation). We suppose that (7.20) $\alpha$ admits a pitchfork bifurcation as described above and consider the nonautonomous differential equation

$$
\dot{x}=f(t, x, \alpha)
$$

depending on a parameter $\alpha$ with a $C^{1}$-function $f:(-\infty, 0) \times\left(x_{-}, x_{+}\right) \times\left(\alpha_{0}, \alpha_{1}\right] \rightarrow \mathbb{R}$. We assume that

$$
\lim _{t \rightarrow-\infty} f(t, x, \alpha)=g(x, \alpha) \quad \text { and } \quad \lim _{t \rightarrow-\infty} D_{2} f(t, x, \alpha)=D_{1} g(x, \alpha)
$$

hold uniformly for all $x \in\left(x_{-}, x_{+}\right)$and $\alpha \in\left(\alpha_{0}, \alpha_{1}\right]$. Then, the following statements are fulfilled:

(i) In case $D_{1} g\left(x_{0}, \alpha_{1}\right)<0$, there exists a continuous function $\mu: D \subset \mathbb{R} \times\left(\alpha_{0}, \alpha_{1}\right] \rightarrow \mathbb{R}$ such that $\mu(\cdot, \alpha)$ is a past attractive solution of $(7.25)_{\alpha}$. We have a total past bifurcation, since

$$
\lim _{\alpha \unlhd \alpha_{0}} d\left(\mathcal{A}_{\mu(\cdot, \alpha)}^{\leftarrow} \mid\{0\}\right)=0
$$

Furthermore, for all $\alpha \in\left(\alpha_{0}, \alpha_{1}\right]$, there exists a past repeller $R(\alpha)$. Due to

$$
\lim _{\alpha \unlhd \alpha_{0}} d\left(\limsup _{t \rightarrow-\infty} R(\alpha, t) \mid\left\{x_{0}\right\}\right)=0,
$$

we also have a past repeller transition.

(ii) In case $D_{1} g\left(x_{0}, \alpha_{1}\right)>0$, there exists a continuous function $\mu: D \subset \mathbb{R} \times\left(\alpha_{0}, \alpha_{1}\right] \rightarrow \mathbb{R}$ such that $\mu(\cdot, \alpha)$ is a past repulsive solution of $(7.25)_{\alpha}$. We have a total past bifurcation, since

$$
\lim _{\alpha \unlhd \alpha_{0}} d\left(\mathcal{R}_{\mu(\cdot, \alpha)}^{\leftarrow} \mid\{0\}\right)=0
$$

Furthermore, for all $\alpha \in\left(\alpha_{0}, \alpha_{1}\right]$, there exists a past attractor $A(\alpha)$. Due to

$$
\lim _{\alpha \searrow \alpha_{0}} d\left(\limsup _{t \rightarrow-\infty} A(\alpha, t) \mid\left\{x_{0}\right\}\right)=0 \text {, }
$$

we also have a past attractor transition. 
Proof. We define the compact intervals $I_{0}:=\left\{\alpha \in\left(\alpha_{0}, \alpha_{1}\right]: h_{1}(\alpha) \leq x_{0}-1\right\}$ and

$$
I_{n}:=\left\{\alpha \in\left(\alpha_{0}, \alpha_{1}\right]: h_{1}(\alpha) \in\left[x_{0}-\frac{1}{n}, x_{0}-\frac{1}{n+1}\right]\right\} \quad \text { for all } n \in \mathbb{N} .
$$

(i) For all $n \in \mathbb{N}_{0}$, we restrict (7.25) $\alpha$ to the parameter area $I_{n}$ and apply Lemma 7.2.1. Therefore, there exists a continuous function $\mu_{n}:\left(-\infty, \tau_{n}\right] \times I_{n} \rightarrow \mathbb{R}$ which describes uniquely determined past attractive solutions. We define

$$
\mu(t, \alpha):=\mu_{n}(t, \alpha) \quad \text { for all } t<0 \text { and } \alpha \in\left(\alpha_{0}, \alpha_{1}\right] \text { with } \alpha \in I_{n} \text { and } t \leq \tau_{n} \text {. }
$$

Due to the uniqueness of the $\mu_{n}$, the so-defined function $\mu: D \rightarrow \mathbb{R}$ for some $D \subset \mathbb{R} \times\left(\alpha_{0}, \alpha_{1}\right]$ is well defined, and the continuity of $\mu$ follows directly. The existence of the past repellers and the limit relations are consequences of Lemma 7.2.1 (i).

(ii) For all $n \in \mathbb{N}_{0}$, we restrict $(7.25)_{\alpha}$ to the parameter area $I_{n}$ and apply Lemma 7.2.4. It is obvious that one can construct a continuous function $\mu: D \subset \mathbb{R} \times\left(\alpha_{0}, \alpha_{1}\right) \rightarrow \mathbb{R}$ which describes past repulsive solutions. The existence of the past attractors and the limit relations are consequences of Lemma 7.2.4 (i).

7.2.7 Theorem (Total Future Bifurcation). We suppose that (7.20) $\alpha$ admits a pitchfork bifurcation as described above and consider the nonautonomous differential equation

$$
\dot{x}=f(t, x, \alpha)
$$

depending on a parameter $\alpha$ with a $C^{1}$-function $f:(0, \infty) \times\left(x_{-}, x_{+}\right) \times\left(\alpha_{0}, \alpha_{1}\right] \rightarrow \mathbb{R}$. We assume that

$$
\lim _{t \rightarrow \infty} f(t, x, \alpha)=g(x, \alpha) \text { and } \lim _{t \rightarrow \infty} D_{2} f(t, x, \alpha)=D_{1} g(x, \alpha)
$$

hold uniformly for all $x \in\left(x_{-}, x_{+}\right)$and $\alpha \in\left(\alpha_{0}, \alpha_{1}\right]$. Then, the following statements are fulfilled:

(i) In case $D_{1} g\left(x_{0}, \alpha_{1}\right)<0$, there exists a continuous function $\mu: D \subset \mathbb{R} \times\left(\alpha_{0}, \alpha_{1}\right] \rightarrow \mathbb{R}$ such that $\mu(\cdot, \alpha)$ is a future attractive solution of $(7.26)_{\alpha}$. We have a total future bifurcation, since

$$
\lim _{\alpha \searrow \alpha_{0}} d\left(\mathcal{A}_{\mu(\cdot, \alpha)} \mid\{0\}\right)=0 .
$$

Furthermore, for all $\alpha \in\left(\alpha_{0}, \alpha_{1}\right]$, there exists a future repeller $R(\alpha)$. Due to

$$
\lim _{\alpha \searrow \alpha_{0}} d\left(\limsup _{t \rightarrow \infty} R(\alpha, t) \mid\left\{x_{0}\right\}\right)=0
$$

we also have a future repeller transition.

(ii) In case $D_{1} g\left(x_{0}, \alpha_{1}\right)>0$, there exists a continuous function $\mu: D \subset \mathbb{R} \times\left(\alpha_{0}, \alpha_{1}\right] \rightarrow \mathbb{R}$ such that $\mu(\cdot, \alpha)$ is a future repulsive solution of $(7.26)_{\alpha}$. We have a total future bifurcation, since

$$
\lim _{\alpha \searrow \alpha_{0}} d\left(\mathcal{R}_{\mu(\cdot, \alpha)} \mid\{0\}\right)=0 .
$$

Furthermore, for all $\alpha \in\left(\alpha_{0}, \alpha_{1}\right]$, there exists a future attractor $A(\alpha)$. Due to 


$$
\lim _{\alpha \unlhd \alpha_{0}} d\left(\limsup _{t \rightarrow \infty} A(\alpha, t) \mid\left\{x_{0}\right\}\right)=0,
$$

we also have a future attractor transition.

PROOF. See proof of Theorem 7.2.6.

In the following example, which is a special case of PötZSCHE \& RASMUSSEN [139, Example 4.1], the center manifold reduction (see, e.g., CARR [38] and HALE [74, Chapter 4] in the autonomous context) is used to verify past and future bifurcations and transitions in an asymptotically autonomous version of the Lorenz system.

7.2.8 EXAMPLE. We consider a nonautonomous version of the famous Lorenz equation (see, e.g., LORENZ [107] and KUZNETSOW [100, pp. 166, 249]), given by the three-dimensional system

$$
\begin{aligned}
& \dot{x}_{1}=\sigma_{\alpha}(t)\left(x_{2}-x_{1}\right) \\
& \dot{x}_{2}=\rho_{\alpha}(t) x_{1}-x_{2}-x_{1} x_{3} \\
& \dot{x}_{3}=-\beta_{\alpha}(t) x_{3}+x_{1} x_{2}
\end{aligned}
$$

In our situation, $\sigma_{\alpha}, \rho_{\alpha}, \beta_{\alpha}$ are perturbed nonautonomously, i.e., we assume that the functions $\sigma_{\alpha}, \rho_{\alpha}, \beta_{\alpha}: \mathbb{R} \rightarrow(0, \infty)$ are given by

$$
\sigma_{\alpha}(t)=\sigma_{0}+\alpha \sigma(t), \quad \rho_{\alpha}(t)=1+\rho_{0}+\alpha \rho(t) \quad \text { and } \quad \beta_{\alpha}(t)=\beta_{0}+\alpha \beta(t) \quad \text { for all } t \in \mathbb{R}
$$

with real constants $\sigma_{0}, \rho_{0}, \beta_{0}>0$, bounded $C^{3}$-functions $\sigma, \rho, \beta$ and $\alpha \in \mathbb{R}$, which will be the bifurcation parameter. It is our goal to study the stability of the equilibrium $x=0$ for different values of $\alpha$. From the linearization of the trivial equilibrium, which is given by

$$
\left(\begin{array}{ccc}
-\sigma_{0} & \sigma_{0} & 0 \\
\rho_{0} & -1 & 0 \\
0 & 0 & -\beta_{0}
\end{array}\right)
$$

we see that in case $\alpha=0$ (i.e., in case of the autonomous Lorenz system), the origin is attractive for $\rho_{0}<0$ and repulsive for $\rho_{0}>0$. More interesting is the nonhyperbolic case $\rho_{0}=0$, where an autonomous pitchfork bifurcation occurs as $\rho_{0}$ passes through 0 (see KUZNETSOW [100, p. 249]). To mimic this situation, we assume $\rho_{0}=0$ from now on. Before proceeding, we formally append the trivial equation $\dot{\alpha}=0$ and-to simplify our calculations-apply the transformation

$$
\left(\begin{array}{l}
y_{1} \\
y_{2} \\
y_{3} \\
y_{4}
\end{array}\right):=\left(\begin{array}{cccc}
-\sigma_{0} & 0 & 1 & 0 \\
1 & 0 & 1 & 0 \\
0 & 1 & 0 & 0 \\
0 & 0 & 0 & 1
\end{array}\right)\left(\begin{array}{l}
x_{1} \\
x_{2} \\
x_{3} \\
\alpha
\end{array}\right) .
$$

This implies the system

$$
\dot{y}=A y+F(t, y)
$$

with $A:=\operatorname{diag}\left(-\sigma_{0}-1,-\beta_{0}, 0,0\right)$ and the nonlinearity

$$
F(t, y):=\left(\begin{array}{c}
\frac{\sigma_{0}}{\sigma_{0}+1} y_{1} y_{2}-\frac{\sigma(t)+\sigma_{0}(\sigma(t)+\rho(t))}{\sigma_{0}+1} y_{1} y_{4}-\frac{1}{\sigma_{0}+1} y_{2} y_{3}+\frac{\rho(t)}{\sigma_{0}+1} y_{3} y_{4} \\
-\sigma_{0} y_{1}^{2}+\left(1-\sigma_{0}\right) y_{1} y_{3}-\beta(t) y_{2} y_{4}+y_{3}^{2}+2 y_{4}^{2} \\
\frac{\sigma_{0}^{2}}{\sigma_{0}+1} y_{1} y_{2}+\frac{\sigma(t)+\sigma_{0}\left(\sigma(t)-\sigma_{0} \rho(t)\right)}{\sigma_{0}+1} y_{1} y_{4}-\frac{\sigma_{0}}{\sigma_{0}+1} y_{2} y_{3}+\frac{\sigma_{0} \rho(t)}{\sigma_{0}+1} y_{3} y_{4} \\
0
\end{array}\right) .
$$


Thus, we can apply Theorem 5.1.3 to (7.27) to show that there exists a local two-dimensional alltime center-unstable manifold $\mathcal{S}^{-}$, given as graph of a function $s^{-}: V \times \mathbb{R} \rightarrow \mathbb{R}^{2}$, where $V \subset \mathbb{R}^{2}$ is a neighborhood of 0 . The ansatz

$$
s^{-}\left(y_{3}, y_{4}, t\right)=\sum_{i=0}^{2} y_{3}^{2-i} y_{4}^{i}\left(\begin{array}{l}
s_{2-i, i}^{1}(t) \\
s_{2-i, i}^{2}(t)
\end{array}\right)+O\left(\sqrt{y_{3}^{2}+y_{4}^{2}}\right)
$$

yields that the equation reduced to the all-time center-unstable manifold $\mathcal{S}^{-}$is given by

$$
\dot{y}_{3}=\frac{\sigma_{0}}{\sigma_{0}+1} \alpha \rho(t) y_{3}-s_{2,0}^{2}(t) y_{3}^{3}+O\left(\alpha y_{3}^{2}, \alpha^{2} y_{3}, y_{3}^{3}\right) \text {. }
$$

Using PÖTZSCHE \& RASMUSSEN [139, Theorem 3.1], we obtain $s_{2,0}^{2}(t) \equiv \frac{1}{\beta_{0}}$, and consequently, the one-dimensional bifurcation equation is given by

$$
\dot{y}_{3}=\frac{\sigma_{0}}{\sigma_{0}+1} \alpha \rho(t) y_{3}-\frac{1}{\beta_{0}} y_{3}^{3}+O\left(\alpha y_{3}^{2}, \alpha^{2} y_{3}, y_{3}^{3}\right) .
$$

We henceforth assume that our system is asymptotically autonomous for $t \rightarrow \pm \infty$, i.e., the limits $t \rightarrow \pm \infty$ of the functions $\sigma, \rho$ and $\beta$ exist. We define

$$
\sigma^{ \pm}:=\lim _{t \rightarrow \pm \infty} \sigma(t), \quad \rho^{ \pm}:=\lim _{t \rightarrow \pm \infty} \rho(t) \quad \text { and } \quad \beta^{ \pm}:=\lim _{t \rightarrow \pm \infty} \beta(t) .
$$

The autonomous limiting equations of the bifurcation equation are then given by

$$
\dot{y}_{3}=\frac{\sigma_{0}}{\sigma_{0}+1} \alpha \rho^{ \pm} y_{3}-\frac{1}{\beta_{0}} y_{3}^{3}+O\left(\alpha y_{3}^{2}, \alpha^{2} y_{3}, y_{3}^{3}\right) \text {. }
$$

It is easy to check that this equation admits a pitchfork bifurcation, i.e., the equilibrium 0 is attractive for $\alpha<0$ and repulsive for $\alpha>0$. For small $\alpha>0$, there are two additional attractive equilibria branching from the origin. One can show that the convergence of the right hand side in (7.28) is uniform in a neighborhood of 0 , and also the derivative with respect to $y_{3}$ of (7.28) converges locally uniformly to the corresponding derivative in (7.29). Thus, Theorem 7.2.6 or Theorem 7.2.7, respectively, is applicable, and therefore, system (7.28) admits a total past or future bifurcation and a past or future attractor transition, respectively. Please note that not only the reduced equation (7.28) gives rise to a nonautonomous transition but also the nonautonomous Lorenz equation itself. This is due to the fact that there exists an asymptotic phase for the center manifold (see AULBACH \& WANNER [22, Theorem 3.3]), i.e., every solution approaches a solution lying in the center manifold in forward time exponentially. Therefore, for small $\alpha>0$, there also exists a past or future attractor of the three-dimensional system, respectively, which shrinks down in the limit $\alpha \triangleleft 0$. However, the three-dimensional nonautonomous Lorenz equation does not admit a past or future bifurcation, since due to the asymptotic phase, the trivial solution is not past repulsive for $\alpha>0$.

To obtain partial nonautonomous bifurcations, we assume that the differential equation (7.20) $\alpha$ admits a supercritical transcritical or saddle node bifurcation at $\left(x_{0}, \alpha_{0}\right)$. This means, there exists a strictly increasing continuous function $h:\left(\alpha_{0}, \alpha_{1}\right) \rightarrow\left(x_{-}, x_{+}\right)$such that for all $\alpha \in\left(\alpha_{0}, \alpha_{1}\right)$, we have

$$
\begin{gathered}
h(\alpha)<x_{0}, \\
g(h(\alpha), \alpha)=g\left(x_{0}, \alpha\right)=0, \\
D_{1} g(h(\alpha), \alpha) \neq 0, D_{1} g\left(x_{0}, \alpha\right) \neq 0 .
\end{gathered}
$$


Moreover, for all $\alpha \in\left(\alpha_{0}, \alpha_{1}\right)$ and $x \in\left(h(\alpha), x_{0}\right)$, the relation $g(x, \alpha) \neq 0$ is satisfied, and we have $\lim _{\alpha \rightarrow \alpha_{0}} h(\alpha)=x_{0}$. Please note that in case of a saddle node bifurcation, one has to transform the greater equilibrium into $x_{0}$. In case of a transcritical bifurcation, we assume that the bigger equilibrium equals $x_{0}$. This can be also reached by a transformation.

7.2.9 Theorem (PARTial Past Bifurcation). We suppose that (7.20) $\alpha$ admits a transcritical or saddle node bifurcation as described above and consider the nonautonomous differential equation

$$
\dot{x}=f(t, x, \alpha)
$$

depending on a parameter $\alpha$ with a $C^{1}$-function $f:(-\infty, 0) \times\left(x_{-}, x_{+}\right) \times\left(\alpha_{0}, \alpha_{1}\right] \rightarrow \mathbb{R}$. We assume that

$$
\lim _{t \rightarrow-\infty} f(t, x, \alpha)=g(x, \alpha) \text { and } \lim _{t \rightarrow-\infty} D_{2} f(t, x, \alpha)=D_{1} g(x, \alpha)
$$

hold uniformly for all $x \in\left(x_{-}, x_{+}\right)$and $\alpha \in\left(\alpha_{0}, \alpha_{1}\right]$. Then, the following statements are fulfilled:

(i) In case $D_{1} g\left(x_{0}, \alpha_{1}\right)<0$, there exists a continuous function $\mu: D \subset \mathbb{R} \times\left(\alpha_{0}, \alpha_{1}\right] \rightarrow \mathbb{R}$ such that $\mu(\cdot, \alpha)$ is a past attractive solution of $(7.30)_{\alpha}$. We have a partial bifurcation, since

$$
\lim _{\alpha \searrow \alpha_{0}} \mathfrak{A}_{\mu(\cdot, \alpha)}^{\leftarrow}=0
$$

(ii) In case $D_{1} g\left(x_{0}, \alpha_{1}\right)>0$, there exists a continuous function $\mu: D \subset \mathbb{R} \times\left(\alpha_{0}, \alpha_{1}\right] \rightarrow \mathbb{R}$ such that $\mu(\cdot, \alpha)$ is a past repulsive solution of $(7.30)_{\alpha}$. We have a partial bifurcation, since

$$
\lim _{\alpha \searrow \alpha_{0}} \mathfrak{R}_{\mu(\cdot, \alpha)}^{\leftarrow}=0
$$

PROOF. See proof of Theorem 7.2.6.

7.2.10 Theorem (PARTial Future Bifurcation). We suppose that (7.20) $\alpha$ admits a transcritical or saddle node bifurcation as described above and consider the nonautonomous differential equation

$$
\dot{x}=f(t, x, \alpha)
$$

depending on a parameter $\alpha$ with a $C^{1}$-function $f:(0, \infty) \times\left(x_{-}, x_{+}\right) \times\left(\alpha_{0}, \alpha_{1}\right] \rightarrow \mathbb{R}$. We assume that

$$
\lim _{t \rightarrow \infty} f(t, x, \alpha)=g(x, \alpha) \text { and } \lim _{t \rightarrow \infty} D_{2} f(t, x, \alpha)=D_{1} g(x, \alpha)
$$

hold uniformly for all $x \in\left(x_{-}, x_{+}\right)$and $\alpha \in\left(\alpha_{0}, \alpha_{1}\right]$. Then, the following statements are fulfilled:

(i) In case $D_{1} g\left(x_{0}, \alpha_{1}\right)<0$, there exists a continuous function $\mu: D \subset \mathbb{R} \times\left(\alpha_{0}, \alpha_{1}\right] \rightarrow \mathbb{R}$ such that $\mu(\cdot, \alpha)$ is a future attractive solution of $(7.31)_{\alpha}$. We have a partial bifurcation, since

$$
\lim _{\alpha \searrow \alpha_{0}} \mathfrak{A}_{\mu(\cdot, \alpha)}=0
$$


(ii) In case $D_{1} g\left(x_{0}, \alpha_{1}\right)>0$, there exists a continuous function $\mu: D \subset \mathbb{R} \times\left(\alpha_{0}, \alpha_{1}\right] \rightarrow \mathbb{R}$ such that $\mu(\cdot, \alpha)$ is a future repulsive solution of $(7.31)_{\alpha}$. We have a partial bifurcation, since

$$
\lim _{\alpha \searrow \alpha_{0}} \mathfrak{R}_{\mu(\cdot, \alpha)}^{\rightarrow}=0
$$

PROOF. See proof of Theorem 7.2.6.

\subsection{Bifurcations in Dimension Two}

In this section, two-dimensional differential equations which exhibit Hopf bifurcations are studied (see, e.g., MARSDEN \& MCCRACKEN [116]). As in the previous section, this bifurcation behavior is transferred to asymptotically autonomous systems.

More precisely, we consider the autonomous differential equation

$$
\begin{aligned}
& \dot{x}=g_{1}(x, y, \alpha) \\
& \dot{y}=g_{2}(x, y, \alpha)
\end{aligned}
$$

depending on a parameter $\alpha$ with a $C^{1}$-function $g:\left(x_{-}, x_{+}\right) \times\left(y_{-}, y_{+}\right) \times\left(\alpha_{0}, \alpha_{1}\right] \rightarrow \mathbb{R}^{2}$ which admits a supercritical Hopf bifurcation at $\left(x_{0}, y_{0}, \alpha_{0}\right)$, i.e., for all $\alpha \in\left(\alpha_{0}, \alpha_{1}\right]$, we have

$$
g\left(x_{0}, y_{0}, \alpha\right)=0 \quad \text { and } \quad D_{(1,2)} g\left(x_{0}, y_{0}, \alpha\right)=\left(\begin{array}{cc}
a(\alpha) & -b(\alpha) \\
b(\alpha) & a(\alpha)
\end{array}\right)
$$

with continuous functions $a:\left(\alpha_{0}, \alpha_{1}\right] \rightarrow \mathbb{R}$ and $b:\left(\alpha_{0}, \alpha_{1}\right] \rightarrow \mathbb{R}$ which fulfill $a(\alpha) \neq 0$ and $b(\alpha) \neq 0$. Furthermore, let $S(\alpha)$ be an attractive (in case $a(\alpha)<0$ ) or a repulsive (in case $a(\alpha)>0)$ periodic orbit of $(7.32)_{\alpha}$, respectively, which depends continuously on $\alpha$ with respect to the Hausdorff distance and converges to $\left(x_{0}, y_{0}\right)$ in the limit $\alpha \rightarrow \alpha_{0}$. We denote the inner area of $S(\alpha)$ by $S^{i}(\alpha)$.

As in the previous section, in the next four lemmata, conditions for the existence of nonautonomous counterparts for the equilibrium $\left(x_{0}, y_{0}\right)$ are studied. In the first instance, the parameter area is restricted to compact subintervals of $\left(\alpha_{0}, \alpha_{1}\right]$.

7.3.1 Lemma (Existence of Past Attractive Solutions). Consider the nonautonomous differential equation

$$
\begin{aligned}
& \dot{x}=f_{1}(t, x, y, \alpha) \\
& \dot{y}=f_{2}(t, x, y, \alpha)
\end{aligned}
$$

depending on a parameter $\alpha$ with a $C^{1}$-function $f:(-\infty, 0) \times\left(x_{-}, x_{+}\right) \times\left(y_{-}, y_{+}\right) \times$ $\left[\alpha_{-}, \alpha_{+}\right] \rightarrow \mathbb{R}^{2}$. We assume that

$$
\lim _{t \rightarrow-\infty} f(t, x, y, \alpha)=g(x, y, \alpha) \quad \text { and } \quad \lim _{t \rightarrow-\infty} D_{(2,3)} f(t, x, y, \alpha)=D_{(1,2)} g(x, y, \alpha)
$$

hold uniformly for all $x \in\left(x_{-}, x_{+}\right), y \in\left(y_{-}, y_{+}\right)$and $\alpha \in\left[\alpha_{-}, \alpha_{+}\right]$. Furthermore, we suppose that 


$$
a(\alpha)<0 \text { for all } \alpha \in\left[\alpha_{-}, \alpha_{+}\right] .
$$

Then, there exist a $\tau<0$ and a continuous function $\mu:(-\infty, \tau] \times\left[\alpha_{-}, \alpha_{+}\right] \rightarrow \mathbb{R}^{2}$ such that $\mu(\cdot, \alpha)$ is the uniquely determined past attractive solution of $(7.33)_{\alpha}$ which fulfills

$$
\lim _{t \rightarrow-\infty} \mu(t, \alpha)=\left(x_{0}, y_{0}\right)
$$

Moreover, we have

$$
\mathcal{A}_{\mu(\cdot, \alpha)}^{\leftarrow}=S^{i}(\alpha)-\left(x_{0}, y_{0}\right) \quad \text { for all } \alpha \in\left[\alpha_{-}, \alpha_{+}\right]
$$

Furthermore, there exists a past repeller $R(\alpha)$ of $(7.33)_{\alpha}$ with

$$
S^{i}(\alpha) \subset \liminf _{t \rightarrow-\infty} R(\alpha, t) \subset \limsup _{t \rightarrow-\infty} R(\alpha, t) \subset \operatorname{cls} S^{i}(\alpha) \quad \text { for all } \alpha \in\left[\alpha_{-}, \alpha_{+}\right] .
$$

ProOF. For simplicity, we assume w.l.o.g. that $\left(x_{0}, y_{0}\right)=(0,0)$ in this proof. The proof is divided into three steps.

STEP 1. There exists a $\tau<0$ and a continuous function $\mu:(-\infty, \tau] \times\left[\alpha_{-}, \alpha_{+}\right] \rightarrow \mathbb{R}^{2}$ such that $\mu(\cdot, \alpha)$ is the uniquely determined past attractive solution of $(7.33)_{\alpha}$ which fulfills $\lim _{t \rightarrow-\infty} \mu(t, \alpha)=(0,0)$.

Due to the compactness of $\left[\alpha_{-}, \alpha_{+}\right]$and the uniform continuity of $g$ on compact sets, there exist $\beta>0$ and $\gamma<0$ with

$$
\frac{\partial g_{1}}{\partial x}(x, y, \alpha) \leq 2 \gamma, \frac{\partial g_{2}}{\partial y}(x, y, \alpha) \leq 2 \gamma \text { and }\left|\frac{\partial g_{1}}{\partial y}(x, y, \alpha)+\frac{\partial g_{2}}{\partial x}(x, y, \alpha)\right| \leq-\gamma
$$

for all $(x, y) \in \operatorname{cls} U_{2 \beta}((0,0))$ and $\alpha \in\left[\alpha_{-}, \alpha_{+}\right]$. This implies the existence of a $\tau<0$ with

$$
\frac{\partial f_{1}}{\partial x}(t, x, y, \alpha) \leq \gamma, \frac{\partial f_{2}}{\partial y}(t, x, y, \alpha) \leq \gamma,\left|\frac{\partial f_{1}}{\partial y}(t, x, y, \alpha)+\frac{\partial f_{2}}{\partial x}(t, x, y, \alpha)\right| \leq-\frac{\gamma}{2}
$$

and

$$
\left|f_{1}(t, 0,0, \alpha)\right|+\left|f_{2}(t, 0,0, \alpha)\right| \leq-\frac{\gamma \beta}{4}
$$

for all $t \leq \tau, x, y \in \operatorname{cls} U_{2 \beta}((0,0))$ and $\alpha \in\left[\alpha_{-}, \alpha_{+}\right]$. For the rest of this step, we fix an $\alpha \in\left[\alpha_{-}, \alpha_{+}\right]$. For all $t \leq \tau$ and $x, y \in \operatorname{cls} U_{2 \beta}((0,0))$, the mean value theorem implies

$$
f(t, x, y, \alpha)=f(t, 0,0, \alpha)+\int_{0}^{1} D_{(2,3)} f(t, \theta x, \theta y, \alpha) \cdot(x, y) d \theta .
$$

It follows that for all $t \leq \tau, \alpha \in\left[\alpha_{-}, \alpha_{+}\right]$and $x, y$ with $x^{2}+y^{2}=\beta^{2}$,

$$
\begin{aligned}
& \langle f(t, x, y, \alpha),(x, y)\rangle=f_{1}(t, x, y, \alpha) x+f_{2}(t, x, y, \alpha) y \\
= & f_{1}(t, 0,0, \alpha) x+f_{2}(t, 0,0, \alpha) y+\int_{0}^{1}\left(\frac{\partial f_{1}}{\partial x}(t, \theta x, \theta y, \alpha) x^{2}+\frac{\partial f_{2}}{\partial y}(t, \theta x, \theta y, \alpha) y^{2}+\right. \\
& \left.\frac{\partial f_{1}}{\partial y}(t, \theta x, \theta y, \alpha) x y+\frac{\partial f_{2}}{\partial x}(t, \theta x, \theta y, \alpha) x y\right) d \theta \\
\leq & -\frac{\gamma \beta^{2}}{4}+\int_{0}^{1}\left(\gamma x^{2}+\gamma y^{2}-\frac{\gamma}{2}|x y|\right) d \theta \leq \frac{\gamma \beta^{2}}{4}<0
\end{aligned}
$$


holds. Therefore, the subset cls $U_{\beta}((0,0))$ of the phase space is forward invariant in the following sense: For all $t^{-} \leq t^{+} \leq \tau$ and $\alpha \in\left[\alpha_{-}, \alpha_{+}\right]$, we have

$$
\lambda\left(t^{+}, t^{-}, \operatorname{cls} U_{\beta}((0,0)), \alpha\right) \subset \operatorname{cls} U_{\beta}((0,0)) .
$$

Thus, the set

$$
\begin{aligned}
M:=\left\{(x, y) \in \operatorname{cls} U_{\beta}((0,0)):\right. & \text { There exists a } t \leq \tau \text { such that } \\
& \|\lambda(t, \tau, x, y, \alpha)\|=\beta \text { and } \\
& \lambda(t, \tau, x, y, \alpha) \neq(\beta, 0) \text { and } \\
& \lambda(t, \tau, x, y, \alpha) \neq(-\beta, 0)\}
\end{aligned}
$$

is nonempty and due to the continuity of the general solution relatively open in in cls $U_{\beta}((0,0))$. This means that $\tilde{M}:=\operatorname{cls} U_{\beta}((0,0)) \backslash M$ is closed. The sets

$$
\begin{array}{r}
M_{1}:=\{(x, y) \in \tilde{M}: \text { There exists a } t \leq \tau \text { such that } \\
\|\lambda(t, \tau, x, y, \alpha)\|=\beta \text { and } \\
\lambda(t, \tau, x, y, \alpha)=(\beta, 0)\}
\end{array}
$$

and

$$
\begin{aligned}
& M_{2}:=\{(x, y) \in \tilde{M}: \text { There exists a } t \leq \tau \text { such that } \\
& \|\lambda(t, \tau, x, y, \alpha)\|=\beta \text { and } \\
& \lambda(t, \tau, x, y, \alpha)=(-\beta, 0)\}
\end{aligned}
$$

are obviously nonempty and due to the continuity of the general solution relatively open in $\tilde{M}$. This implies that $M_{1} \cup M_{2} \subsetneq \tilde{M}$. Therefore, there exists a $(\hat{x}, \hat{y}) \in U_{\beta}(0,0)$ with

$$
\mu(t, \alpha):=\lambda(t, \tau, \hat{x}, \hat{y}, \alpha) \in U_{\beta}((0,0)) \quad \text { for all } t \leq \tau .
$$

To show that $\mu$ is past attractive, we study the differential equation of the perturbed motion

$$
\begin{aligned}
& \dot{x}=h_{1}(t, x, y, \alpha):=f_{1}\left(t, x+\mu_{1}(t, \alpha), y+\mu_{2}(t, \alpha), \alpha\right)-f_{1}(t, \mu(t, \alpha), \alpha) \\
& \dot{y}=h_{2}(t, x, y, \alpha):=f_{2}\left(t, x+\mu_{1}(t, \alpha), y+\mu_{2}(t, \alpha), \alpha\right)-f_{2}(t, \mu(t, \alpha), \alpha)
\end{aligned}
$$

Due to the mean value theorem, for all $t \leq \tau$ and $(x, y) \in U_{\beta}((0,0))$,

$$
\begin{aligned}
h(t, x, y, \alpha) & =\int_{0}^{1} D_{(2,3)} h(t, \theta x, \theta y, \alpha) \cdot(x, y) d \theta \\
& =\int_{0}^{1} D_{(2,3)} f\left(t, \theta x+\mu_{1}(t, \alpha), \theta y+\mu_{2}(t, \alpha), \alpha\right) \cdot(x, y) d \theta
\end{aligned}
$$

is fulfilled. Thus, for all $(r, \phi) \in(0, \beta) \times[0,2 \pi)$ and $t \leq \tau$, we have

$$
\begin{gathered}
h_{1}(t, r \cos \phi, r \sin \phi, \alpha) \cos \phi+h_{2}(t, r \cos \phi, r \sin \phi, \alpha) \sin \phi \\
=\int_{0}^{1}\left(\frac{\partial f_{1}}{\partial x}\left(t, \theta r \cos \phi+\mu_{1}(t, \alpha), \theta r \sin \phi+\mu_{2}(t, \alpha), \alpha\right) r \cos ^{2} \phi+\right. \\
\frac{\partial f_{2}}{\partial y}\left(t, \theta r \cos \phi+\mu_{1}(t, \alpha), \theta r \sin \phi+\mu_{2}(t, \alpha), \alpha\right) r \sin ^{2} \phi+
\end{gathered}
$$




$$
\begin{gathered}
\frac{\partial f_{1}}{\partial y}\left(t, \theta r \cos \phi+\mu_{1}(t, \alpha), \theta r \sin \phi+\mu_{2}(t, \alpha), \alpha\right) r \cos \phi \sin \phi+ \\
\left.\frac{\partial f_{2}}{\partial x}\left(t, \theta r \cos \phi+\mu_{1}(t, \alpha), \theta r \sin \phi+\mu_{2}(t, \alpha), \alpha\right) r \cos \phi \sin \phi\right) d \theta \\
\leq \int_{0}^{1}\left(\gamma r \cos ^{2} \phi+\gamma r \sin ^{2} \phi-\frac{\gamma}{2} r \cos \phi \sin \phi\right) d \theta \leq r \frac{\gamma}{2} .
\end{gathered}
$$

Applying polar coordinates (see AulBACH [14, Satz 5.2.1, p. 192]), we see that $\mu(\cdot, \alpha)$ is past attractive. Moreover, the limit relation $\lim _{t \rightarrow-\infty} \mu(t, \alpha)=(0,0)$ is obviously satisfied. The uniqueness of $\mu(\cdot, \alpha)$ follows directly from Proposition 2.1.27 (i).

STEP 2. $\mu$ is continuous.

See Step 2 of the proof of Lemma 7.2.1.

STEP 3. The assertions concerning $\mathcal{A}_{\mu(\cdot, \alpha)}^{\leftarrow}$ and the past repellers are fulfilled.

This follows directly from Lemma 7.1.5.

\subsubsection{Lemma (Existence of Future Attractive Solutions). Consider the nonau-} tonomous differential equation

$$
\begin{aligned}
& \dot{x}=f_{1}(t, x, y, \alpha) \\
& \dot{y}=f_{2}(t, x, y, \alpha)
\end{aligned}
$$

depending on a parameter $\alpha$ with a $C^{1}$-function $f:(0, \infty) \times\left(x_{-}, x_{+}\right) \times\left(y_{-}, y_{+}\right) \times$ $\left[\alpha_{-}, \alpha_{+}\right] \rightarrow \mathbb{R}^{2}$. We assume that

$$
\lim _{t \rightarrow \infty} f(t, x, y, \alpha)=g(x, y, \alpha) \quad \text { and } \quad \lim _{t \rightarrow \infty} D_{(2,3)} f(t, x, y, \alpha)=D_{(1,2)} g(x, y, \alpha)
$$

hold uniformly for all $x \in\left(x_{-}, x_{+}\right), y \in\left(y_{-}, y_{+}\right)$and $\alpha \in\left[\alpha_{-}, \alpha_{+}\right]$. Furthermore, we suppose that

$$
a(\alpha)<0 \text { for all } \alpha \in\left[\alpha_{-}, \alpha_{+}\right] .
$$

Then, there exist $\tau>0$ and $\beta>0$ such that for $(x, y) \in U_{\beta}\left(\left(x_{0}, y_{0}\right)\right)$ and $\alpha \in\left[\alpha_{-}, \alpha_{+}\right]$, the solution $\lambda(\cdot, \tau, x, y, \alpha)$ of $(7.34)_{\alpha}$ is future attractive with

$$
\lim _{t \rightarrow \infty} \lambda(t, \tau, x, y, \alpha)=\left(x_{0}, y_{0}\right)
$$

and

$$
\mathcal{A}_{\lambda(\cdot, \tau, x, y, \alpha)}=S^{i}(\alpha)-\left(x_{0}, y_{0}\right) .
$$

Furthermore, there exists a future repeller $R(\alpha)$ of $(7.34)_{\alpha}$ with

$$
S^{i}(\alpha) \subset \liminf _{t \rightarrow \infty} R(\alpha, t) \subset \limsup _{t \rightarrow \infty} R(\alpha, t) \subset \operatorname{cls} S^{i}(\alpha) \quad \text { for all } \alpha \in\left[\alpha_{-}, \alpha_{+}\right] .
$$

Proof. For simplicity, we assume w.l.o.g. that $\left(x_{0}, y_{0}\right)=(0,0)$ in this proof. Due to the compactness of $\left[\alpha_{-}, \alpha_{+}\right]$and the uniform continuity of $g$ on compact sets, there exist $\beta>0$ and $\gamma<0$ with

$$
\frac{\partial g_{1}}{\partial x}(x, y, \alpha) \leq 2 \gamma, \frac{\partial g_{2}}{\partial y}(x, y, \alpha) \leq 2 \gamma \text { and }\left|\frac{\partial g_{1}}{\partial y}(x, y, \alpha)+\frac{\partial g_{2}}{\partial x}(x, y, \alpha)\right| \leq-\gamma
$$


for all $(x, y) \in \operatorname{cls} U_{2 \beta}((0,0))$ and $\alpha \in\left[\alpha_{-}, \alpha_{+}\right]$. This implies the existence of a $\tau<0$ with

$$
\frac{\partial f_{1}}{\partial x}(t, x, y, \alpha) \leq \gamma, \frac{\partial f_{2}}{\partial y}(t, x, y, \alpha) \leq \gamma,\left|\frac{\partial f_{1}}{\partial y}(t, x, y, \alpha)+\frac{\partial f_{2}}{\partial x}(t, x, y, \alpha)\right| \leq-\frac{\gamma}{2}
$$

and

$$
\left|f_{1}(t, 0,0, \alpha)\right|+\left|f_{2}(t, 0,0, \alpha)\right| \leq-\frac{\gamma \beta}{4}
$$

for all $t \geq \tau, x, y \in \operatorname{cls} U_{2 \beta}((0,0))$ and $\alpha \in\left[\alpha_{-}, \alpha_{+}\right]$. For the rest of this proof, we fix an $\alpha \in\left[\alpha_{-}, \alpha_{+}\right]$. For all $t \geq \tau$ and $x, y \in \operatorname{cls} U_{2 \beta}((0,0))$, the mean value theorem implies

$$
f(t, x, y, \alpha)=f(t, 0,0, \alpha)+\int_{0}^{1} f_{x}(t, \theta x, \theta y, \alpha) \cdot(x, y) d \theta .
$$

Thus, for all $t \geq \tau$ and $x, y$ with $x^{2}+y^{2}=\beta^{2}$, we have

$$
\begin{aligned}
& \langle f(t, x, y, \alpha),(x, y))=f_{1}(t, x, y, \alpha) x+f_{2}(t, x, y, \alpha) y \\
= & f_{1}(t, 0,0, \alpha) x+f_{2}(t, 0,0, \alpha) y+\int_{0}^{1}\left(\frac{\partial f_{1}}{\partial x}(t, \theta x, \theta y, \alpha) x^{2}+\frac{\partial f_{2}}{\partial y}(t, \theta x, \theta y, \alpha) y^{2}+\right. \\
& \left.\frac{\partial f_{1}}{\partial y}(t, \theta x, \theta y, \alpha) x y+\frac{\partial f_{2}}{\partial x}(t, \theta x, \theta y, \alpha) x y\right) d \theta \\
\leq & -\frac{\gamma \beta^{2}}{4}+\int_{0}^{1}\left(\gamma x^{2}+\gamma y^{2}-\frac{\gamma}{2}|x y|\right) d \theta \leq \frac{\gamma \beta^{2}}{4}<0 .
\end{aligned}
$$

Therefore, the subset cls $U_{\beta}((0,0))$ of the phase space is forward invariant in the following sense: For all $t^{+} \geq t^{-} \geq \tau$, we have

$$
\lambda\left(t^{+}, t^{-}, \operatorname{cls} U_{\beta}((0,0)), \alpha\right) \subset \operatorname{cls} U_{\beta}((0,0)) .
$$

We choose $(\hat{x}, \hat{y}) \in U_{\beta}((0,0))$ arbitrarily and consider for the rest of this proof in particular the solution $v(\cdot):=\lambda(\cdot, \tau, \hat{x}, \hat{y}, \alpha)$ on the interval $[\tau, \infty)$. It is obvious that $\lim _{t \rightarrow-\infty} v(t)=(0,0)$ holds. To show that $v$ is future attractive, we study the differential equation of the perturbed motion

$$
\begin{aligned}
& \dot{x}=h_{1}(t, x, y, \alpha):=f_{1}\left(t, x+v_{1}(t), y+v_{2}(t), \alpha\right)-f_{1}(t, v(t), \alpha) \\
& \dot{y}=h_{2}(t, x, y, \alpha):=f_{2}\left(t, x+v_{1}(t), y+v_{2}(t), \alpha\right)-f_{2}(t, v(t), \alpha)
\end{aligned}
$$

Due to the mean value theorem, we have for all $t \geq \tau$ and $(x, y) \in U_{\beta}((0,0))$,

$$
\begin{aligned}
h(t, x, y, \alpha)= & \int_{0}^{1} D_{(2,3)} h(t, \theta x, \theta y, \alpha) \cdot(x, y) d \theta= \\
& \int_{0}^{1} D_{(2,3)} f\left(t, \theta x+\mu_{1}(t, \alpha), \theta y+\mu_{2}(t, \alpha), \alpha\right) \cdot(x, y) d \theta .
\end{aligned}
$$

Thus, for all $(r, \phi) \in(0, \beta) \times[0,2 \pi)$ and $t \geq \tau$, we have

$$
\begin{gathered}
h_{1}(t, r \cos \phi, r \sin \phi, \alpha) \cos \phi+h_{2}(t, r \cos \phi, r \sin \phi, \alpha) \sin \phi \\
=\int_{0}^{1}\left(\frac{\partial f_{1}}{\partial x}\left(t, \theta r \cos \phi+\mu_{1}(t, \alpha), \theta r \sin \phi+\mu_{2}(t, \alpha), \alpha\right) r \cos ^{2} \phi+\right. \\
\frac{\partial f_{2}}{\partial y}\left(t, \theta r \cos \phi+\mu_{1}(t, \alpha), \theta r \sin \phi+\mu_{2}(t, \alpha), \alpha\right) r \sin ^{2} \phi+
\end{gathered}
$$




$$
\begin{gathered}
\frac{\partial f_{1}}{\partial y}\left(t, \theta r \cos \phi+\mu_{1}(t, \alpha), \theta r \sin \phi+\mu_{2}(t, \alpha), \alpha\right) r \cos \phi \sin \phi+ \\
\left.\frac{\partial f_{2}}{\partial x}\left(t, \theta r \cos \phi+\mu_{1}(t, \alpha), \theta r \sin \phi+\mu_{2}(t, \alpha), \alpha\right) r \cos \phi \sin \phi\right) d \theta \\
\leq \int_{0}^{1}\left(\gamma r \cos ^{2} \phi+\gamma r \sin ^{2} \phi-\frac{\gamma}{2} r \cos \phi \sin \phi\right) d \theta \leq r \frac{\gamma}{2} .
\end{gathered}
$$

Applying polar coordinates, we see that $v$ is future attractive. The asserted relations concerning $\mathcal{A}_{\mu(\cdot, \alpha)}$ and the existence of the future repellers follow directly from Lemma 7.1.6.

7.3.3 Lemma (EXISTEnCE of PASt Repulsive Solutions). Consider the nonautonomous differential equation

$$
\begin{aligned}
\dot{x} & =f_{1}(t, x, y, \alpha) \\
\dot{y} & =f_{2}(t, x, y, \alpha)
\end{aligned}
$$

depending on a parameter $\alpha$ with a $C^{1}$-function $f:(-\infty, 0) \times\left(x_{-}, x_{+}\right) \times\left(y_{-}, y_{+}\right) \times$ $\left[\alpha_{-}, \alpha_{+}\right] \rightarrow \mathbb{R}^{2}$. We assume that

$$
\lim _{t \rightarrow-\infty} f(t, x, y, \alpha)=g(x, y, \alpha) \quad \text { and } \quad \lim _{t \rightarrow-\infty} D_{(2,3)} f(t, x, y, \alpha)=D_{(1,2)} g(x, y, \alpha)
$$

hold uniformly for all $x \in\left(x_{-}, x_{+}\right), y \in\left(y_{-}, y_{+}\right)$and $\alpha \in\left[\alpha_{-}, \alpha_{+}\right]$. Furthermore, we suppose that

$$
a(\alpha)>0 \text { for all } \alpha \in\left[\alpha_{-}, \alpha_{+}\right] .
$$

Then, there exist $\tau<0$ and $\beta>0$ such that for $(x, y) \in U_{\beta}\left(\left(x_{0}, y_{0}\right)\right)$ and $\alpha \in\left[\alpha_{-}, \alpha_{+}\right]$, the solution $\lambda(\cdot, \tau, x, y, \alpha)$ of $(7.35)_{\alpha}$ is past repulsive with

$$
\lim _{t \rightarrow-\infty} \lambda(t, \tau, x, y, \alpha)=\left(x_{0}, y_{0}\right)
$$

and

$$
\mathcal{R}_{\lambda(\cdot, \tau, x, y, \alpha)}^{\leftarrow}=S^{i}(\alpha)-\left(x_{0}, y_{0}\right)
$$

Furthermore, there exists a past attractor $A(\alpha)$ of $(7.35)_{\alpha}$ with

$$
S^{i}(\alpha) \subset \liminf _{t \rightarrow-\infty} A(\alpha, t) \subset \limsup _{t \rightarrow-\infty} A(\alpha, t) \subset \operatorname{cls} S^{i}(\alpha) \quad \text { for all } \alpha \in\left[\alpha_{-}, \alpha_{+}\right] .
$$

PROOF. The assertions follow directly from Proposition 2.1.22 and Lemma 7.3.2.

7.3.4 Lemma (Existence of Future Repulsive Solutions). Consider the nonautonomous differential equation

$$
\begin{aligned}
\dot{x} & =f_{1}(t, x, y, \alpha) \\
\dot{y} & =f_{2}(t, x, y, \alpha)
\end{aligned}
$$

depending on a parameter $\alpha$ with a $C^{1}$-function $f:(0, \infty) \times\left(x_{-}, x_{+}\right) \times\left(y_{-}, y_{+}\right) \times$ $\left[\alpha_{-}, \alpha_{+}\right] \rightarrow \mathbb{R}^{2}$. We assume that 


$$
\lim _{t \rightarrow \infty} f(t, x, y, \alpha)=g(x, y, \alpha) \quad \text { and } \quad \lim _{t \rightarrow \infty} D_{(2,3)} f(t, x, y, \alpha)=D_{(1,2)} g(x, y, \alpha)
$$

hold uniformly for all $x \in\left(x_{-}, x_{+}\right), y \in\left(y_{-}, y_{+}\right)$and $\alpha \in\left[\alpha_{-}, \alpha_{+}\right]$. Furthermore, we suppose that

$$
a(\alpha)>0 \quad \text { for all } \alpha \in\left[\alpha_{-}, \alpha_{+}\right] .
$$

Then, there exist a $\tau>0$ and a continuous function $\mu:[\tau, \infty) \times\left[\alpha_{-}, \alpha_{+}\right] \rightarrow \mathbb{R}^{2}$ such that $\mu(\cdot, \alpha)$ is the uniquely determined future repulsive solution of $(7.36)_{\alpha}$ which fulfills $\lim _{t \rightarrow \infty} \mu(t, \alpha)=\left(x_{0}, y_{0}\right)$. Moreover, we have

$$
\mathcal{R}_{\mu(\cdot, \alpha)}=S^{i}(\alpha)-\left(x_{0}, y_{0}\right) \quad \text { for all } \alpha \in\left[\alpha_{-}, \alpha_{+}\right] .
$$

Furthermore, there exists a future attractor $A(\alpha)$ of $(7.36)_{\alpha}$ with

$$
S^{i}(\alpha) \subset \liminf _{t \rightarrow \infty} A(\alpha, t) \subset \limsup _{t \rightarrow \infty} A(\alpha, t) \subset \operatorname{cls} S^{i}(\alpha) \quad \text { for all } \alpha \in\left[\alpha_{-}, \alpha_{+}\right] .
$$

PROOF. The assertions follow directly from Proposition 2.1.22 and Lemma 7.3.1.

As in the previous subsection, these four lemmata lead to the existence of total nonautonomous bifurcations and transitions.

7.3.5 TheOREM (PAST Hopf Bifurcation). We suppose that (7.32) $)_{\alpha}$ admits a Hopf bifurcation as described above and consider the nonautonomous differential equation

$$
\dot{x}=f(t, x, \alpha)
$$

depending on a parameter $\alpha$ with a $C^{1}$-function $f:(-\infty, 0) \times\left(x_{-}, x_{+}\right) \times\left(y_{-}, y_{+}\right) \times$ $\left(\alpha_{0}, \alpha_{1}\right] \rightarrow \mathbb{R}^{2}$. We assume that

$$
\lim _{t \rightarrow-\infty} f(t, x, y, \alpha)=g(x, y, \alpha) \quad \text { and } \quad \lim _{t \rightarrow-\infty} D_{(2,3)} f(t, x, y, \alpha)=D_{(1,2)} g(x, y, \alpha)
$$

hold uniformly for all $x \in\left(x_{-}, x_{+}\right), y \in\left(y_{-}, y_{+}\right)$and $\alpha \in\left(\alpha_{0}, \alpha_{1}\right]$. Then, the following statements are fulfilled:

(i) In case $a\left(\alpha_{1}\right)<0$, there exists a continuous function $\mu: D \subset \mathbb{R} \times\left(\alpha_{0}, \alpha_{1}\right] \rightarrow \mathbb{R}^{2}$ such that $\mu(\cdot, \alpha)$ is a past attractive solution of $(7.37)_{\alpha}$. We have a total past bifurcation, since

$$
\lim _{\alpha \searrow \alpha_{0}} d\left(\mathcal{A}_{\mu(\cdot, \alpha)}^{\leftarrow} \mid\{0\}\right)=0
$$

Furthermore, for all $\alpha \in\left(\alpha_{0}, \alpha_{1}\right]$, there exists a past repeller $R(\alpha)$. We also have a past repeller transition, since

$$
\lim _{\alpha \searrow \alpha_{0}} d\left(\limsup _{t \rightarrow-\infty} R(\alpha, t) \mid\left\{\left(x_{0}, y_{0}\right)\right\}\right)=0
$$

(ii) In case $a\left(\alpha_{1}\right)>0$, there exists a continuous function $\mu: D \subset \mathbb{R} \times\left(\alpha_{0}, \alpha_{1}\right] \rightarrow \mathbb{R}^{2}$ such that $\mu(\cdot, \alpha)$ is a past repulsive solution of $(7.37)_{\alpha}$. We have a total past bifurcation, since 


$$
\lim _{\alpha \searrow \alpha_{0}} d\left(\mathcal{R}_{\mu(\cdot, \alpha)}^{\leftarrow} \mid\{0\}\right)=0
$$

Furthermore, for all $\alpha \in\left(\alpha_{0}, \alpha_{1}\right]$, there exists a past attractor $A(\alpha)$. We also have a past attractor transition, since

$$
\lim _{\alpha \unlhd \alpha_{0}} d\left(\limsup _{t \rightarrow-\infty} A(\alpha, t) \mid\left\{\left(x_{0}, y_{0}\right)\right\}\right)=0 .
$$

PROOF. See proof of Theorem 7.2.6.

7.3.6 TheOREM (Future Hopf Bifurcation). We suppose that (7.32) $\alpha$ admits a Hopf bifurcation as described above and consider the nonautonomous differential equation

$$
\dot{x}=f(t, x, \alpha)
$$

depending on a parameter $\alpha$ with a $C^{1}$-function $f:(0, \infty) \times\left(x_{-}, x_{+}\right) \times\left(y_{-}, y_{+}\right) \times\left(\alpha_{0}, \alpha_{1}\right] \rightarrow$ $\mathbb{R}^{2}$. We assume that

$$
\lim _{t \rightarrow \infty} f(t, x, y, \alpha)=g(x, y, \alpha) \quad \text { and } \quad \lim _{t \rightarrow \infty} D_{(2,3)} f(t, x, y, \alpha)=D_{(1,2)} g(x, y, \alpha)
$$

hold uniformly for all $x \in\left(x_{-}, x_{+}\right), y \in\left(y_{-}, y_{+}\right)$and $\alpha \in\left(\alpha_{0}, \alpha_{1}\right]$. Then, the following statements are fulfilled:

(i) In case $a\left(\alpha_{1}\right)<0$, there exists a continuous function $\mu: D \subset \mathbb{R} \times\left(\alpha_{0}, \alpha_{1}\right] \rightarrow \mathbb{R}^{2}$ such that $\mu(\cdot, \alpha)$ is a future attractive solution of $(7.38)_{\alpha}$. We have a total future bifurcation, since

$$
\lim _{\alpha \searrow \alpha_{0}} d\left(\overrightarrow{\mathcal{A}_{\mu(\cdot, \alpha)}} \mid\{0\}\right)=0 .
$$

Furthermore, for all $\alpha \in\left(\alpha_{0}, \alpha_{1}\right]$, there exists a future repeller $R(\alpha)$. We also have a future repeller transition, since

$$
\lim _{\alpha \unlhd \alpha_{0}} d\left(\limsup _{t \rightarrow \infty} R(\alpha, t) \mid\left\{\left(x_{0}, y_{0}\right)\right\}\right)=0 .
$$

(ii) In case $a\left(\alpha_{1}\right)>0$, there exists a continuous function $\mu: D \subset \mathbb{R} \times\left(\alpha_{0}, \alpha_{1}\right] \rightarrow \mathbb{R}^{2}$ such that $\mu(\cdot, \alpha)$ is a future repulsive solution of $(7.38)_{\alpha}$. We have a total future bifurcation, since

$$
\lim _{\alpha \searrow \alpha_{0}} d\left(\mathcal{R}_{\mu(\cdot, \alpha)} \mid\{0\}\right)=0 .
$$

Furthermore, for all $\alpha \in\left(\alpha_{0}, \alpha_{1}\right]$, there exists a future attractor $A(\alpha)$. We also have a future attractor transition, since

$$
\lim _{\alpha \searrow \alpha_{0}} d\left(\limsup _{t \rightarrow \infty} A(\alpha, t) \mid\left\{\left(x_{0}, y_{0}\right)\right\}\right)=0 \text {. }
$$

PROOF. See proof of Theorem 7.2.6. 
This chapter is concluded with the following famous example.

7.3.7 EXAMPLE. We consider a nonautonomous version of the unforced Duffing-van der Pol equation

$$
\begin{aligned}
& \dot{x_{1}}=x_{2} \\
& \dot{x_{2}}=-x_{1}+\alpha \beta(t) x_{2}-x_{1}^{2}\left(x_{1}+x_{2}\right)
\end{aligned}
$$

depending on a real parameter $\alpha$. We assume that $\beta: \mathbb{R} \rightarrow \mathbb{R}^{+}$is a $C^{1}$-function. This differential equation describes a nonlinear oscillator. It is well-known (see, e.g., HOLMES \& RAND [81] or MARSDEN \& MCCRACKEN [116]) that in case the function $\beta$ is constant and positive, i.e., the system is autonomous, the equilibrium $(0,0)$ is attractive for $\alpha<0$. At $\alpha=0$, the system undergoes a Hopf bifurcation: The equilibrium $(0,0)$ becomes repulsive and an attractive periodic orbit appears. As a consequence, we also have a bifurcation of autonomous attractors (see AULBACH \& RASMUSSEN \& SIEGMUND [16]): For values $\alpha \leq 0$, the singleton $\{(0,0)\}$ is an attractor. If $\alpha$ is small and positive, then the interior of the bifurcating periodic orbit is an attractor of the system.

We assume that the nonautonomous system is past (future, respectively) asymptotically autonomous, i.e., the function $\beta$ fulfills

$$
\bar{\beta}:=\lim _{t \rightarrow \pm \infty} \beta(t)>0 .
$$

Then, the differential equation fulfills the hypotheses of Theorem 7.3.5 or Theorem 7.3.6 in some neighborhood of $(0,0)$, respectively, and we have a nonautonomous bifurcation and transition as described in these theorems. 


\section{APPENDIX}

This supplementary appendix contains well-known definitions and results used in this thesis which — to provide reading fluency—are not stated before.

The first section of this appendix is devoted to fundamental facts about ordinary differential equations. In Section A.2, some useful lemmata are stated, and in the last section, basic properties of projective spaces are treated.

\section{A.1 OrdinARY DifFERENTIAL EQUATIONS}

We begin with the definition of an ordinary differential equation in the Euclidian space $\mathbb{R}^{N}$.

A.1.1 Definition (Ordinary Differential Equation). For given $N, M \in \mathbb{N}$, let $D \subset \mathbb{R} \times \mathbb{R}^{N} \times \mathbb{R}^{M}$ be an open set and $f: D \rightarrow \mathbb{R}^{N}$ be a function. Then, the equation

$$
\dot{x}=f(t, x, \alpha)
$$

is called (nonautonomous) ordinary differential equation which depends on a parameter $\alpha$. For fixed $\hat{\alpha} \in \mathbb{R}^{M}$, we say that a differentiable function $\mu: \llbracket \rightarrow \mathbb{R}^{N}$, $\llbracket$ an open interval, is a solution of $(A .1)_{\hat{\alpha}}$ if $(t, \mu(t), \hat{\alpha}) \in D$ for all $t \in \mathbb{Q}$ and

$$
\dot{\mu}(t):=\frac{d \mu}{d t}(t)=f(t, \mu(t), \hat{\alpha}) \quad \text { for all } t \in \mathbb{\square}
$$

is fulfilled. The combination of the differential equation $(A .1)_{\hat{\alpha}}$ and an initial value condition $x(\tau)=\xi$ is called initial value problem. We say, a solution $\mu$ of $(A .1)_{\hat{\alpha}}$ solves this initial value problem if $\mu(\tau)=\xi$.

For the uniqueness of solutions of ordinary differential equations, the concept of Lipschitz continuity is appropriate.

A.1.2 Definition (Lipschitz Continuous Functions). For given $N, M \in \mathbb{N}$, let $D \subset \mathbb{R}^{1+N+M}$ and $g: D \rightarrow \mathbb{R}^{N}$ be a function. We say that $g$ is (globally) Lipschitz continuous if there exists a constant $L \geq 0$ with

$$
\|g(t, x, \alpha)-g(t, y, \alpha)\| \leq L\|x-y\| \quad \text { for all }(t, x, \alpha),(t, y, \alpha) \in D \text {. }
$$


$g$ is called locally Lipschitz continuous if for all $(t, x, \alpha) \in D$, there exist neighborhoods $V$ of $t$ and $W$ of $\alpha$ such that the restriction of $g$ to $V \times W \times\{\alpha\}$ is globally Lipschitz continuous.

The proof of the following proposition can be found, e.g., in AULBACH [14, Definition 2.6.2, Satz 7.2.2].

A.1.3 Proposition (General Solution). Let $N, M \in \mathbb{N}, D \subset \mathbb{R} \times \mathbb{R}^{N} \times \mathbb{R}^{M}$ be open and $f: D \rightarrow \mathbb{R}^{N}$ be a locally Lipschitz continuous function, and consider the nonautonomous differential equation $(A .1)_{\alpha}$. Then, there exist an open set $\Omega \subset \mathbb{R} \times \mathbb{R} \times \mathbb{R}^{N} \times \mathbb{R}^{M}$ and a continuous function $\lambda: \Omega \rightarrow \mathbb{R}^{N}$ such that for fixed $(\tau, \xi, \hat{\alpha}) \in D$, the function $\lambda(\cdot, \tau, \xi, \hat{\alpha})$ is a non-continuable solution of the initial value problem $(A .1)_{\hat{\alpha}}, x(\tau)=\xi$. The function $\lambda$ is called the general solution of $(A .1)_{\alpha}$.

A.1.4 REMARK. In case the differential equation $(A .1)_{\alpha}$ does not depend on $\alpha$, the fourth argument of the general solution is omitted.

A.1.5 Definition (TRANSITION OpERATOR). Let $\square \subset \mathbb{R}$ be an interval, and consider the nonautonomous linear differential equation

$$
\dot{x}=A(t) x
$$

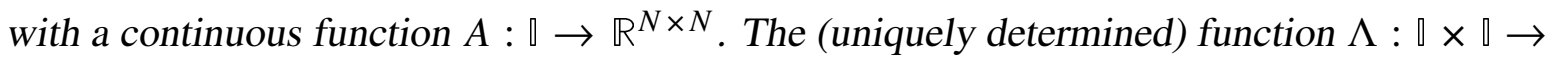
$\mathbb{R}^{N \times N}$ with

$$
\Lambda(t, \tau) \xi=\lambda(t, \tau, \xi) \quad \text { for all } t, \tau \in \mathbb{a} \text { and } \xi \in \mathbb{R}^{N},
$$

where $\lambda$ denotes the general solution of (A.2), is called transition operator of (A.2). In case (A.2) is autonomous, i.e., $A=A(t)$ for all $t \in \mathbb{\square}=\mathbb{R}$ with a matrix $A \in \mathbb{R}^{N \times N}$, we define the matrix exponential function $e^{A \cdot}: \mathbb{R} \rightarrow \mathbb{R}^{N \times N}$ by

$$
e^{A t}:=\Lambda(t, 0) \quad \text { for all } t \in \mathbb{R} \text {. }
$$

Inhomogeneous linear differential equations are solved by the variation of constants formula.

A.1.6 Proposition (VAriation of Constants Formula). Let $\square \subset \mathbb{R}$ be an interval, and consider the nonautonomous inhomogeneous linear differential equation

$$
\dot{x}=A(t) x+b(t)
$$

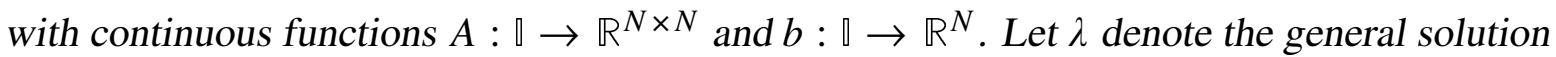
of (A.3) and $\Lambda$ denote the transition operator of $\dot{x}=A(t) x$. Then, we have the representation

$$
\lambda(t, \tau, \xi)=\Lambda(t, \tau) \xi+\int_{\tau}^{t} \Lambda(t, s) b(s) d s \quad \text { for all } t, \tau \in \mathbb{a} \text { and } \xi \in \mathbb{R}^{N}
$$

This equation is called the variation of constants formula.

Proof. See, e.g., Coppel [53, p. 45]. 
For the analysis in the vicinity of a given reference solution, the differential equation of perturbed motion is of great importance.

A.1.7 Proposition (Differential Equation of Perturbed Motion). For given $D \subset \mathbb{R} \times \mathbb{R}^{N}$, let $f: D \rightarrow \mathbb{R}^{N}$ be a locally Lipschitz continuous function, and consider the nonautonomous differential equation

$$
\dot{x}=f(t, x)
$$

with a solution $\lambda: \llbracket \rightarrow \mathbb{R}^{N}, \square$ an interval. Then, the so-called differential equation of perturbed motion

$$
\dot{x}=f(t, x+\lambda(t))-f(t, \lambda(t))
$$

has the following properties:

(i) If $v: \mathbb{l} \rightarrow \mathbb{R}^{N}$ is a solution of (A.4) and $\sqrt{ } \subset \mathbb{}$, then $\mu:=v-\lambda$ is a solution of (A.5) on $\sqrt{ }$.

(ii) If $\mu: \sqrt{ } \rightarrow \mathbb{R}^{N}$ is a solution of (A.5) and $\sqrt{ } \subset \mathbb{q}$, then $v:=\mu+\lambda$ is a solution of (A.4) on $\sqrt{ }$.

\section{A.2 UsEFul LEMMATA}

The following lemma, which goes back to GRONWALL [70], plays a central role in obtaining estimates for solutions of differential equations.

A.2.1 Lemma (GRONWALL's InequAlity). Let $a \geq 0$ and $u, b:\left[\tau_{-}, \tau_{+}\right] \rightarrow \mathbb{R}_{0}^{+}$be continuous functions, and suppose that

$$
u(t) \leq a+\int_{\tau_{-}}^{t} b(s) u(s) d s \quad \text { for all } t \in\left[\tau_{-}, \tau_{+}\right]
$$

is fulfilled. Then,

$$
u(t) \leq a \exp \left(\int_{\tau_{-}}^{t} b(s) d s\right) \quad \text { for all } t \in\left[\tau_{-}, \tau_{+}\right]
$$

Proof. See, e.g., Abraham \& Marsden \& Ratiu [1, Theorem 4.1.7, p. 242].

The following lemma provides a triangle inequality for the Hausdorff-semi distance, which has been introduced in Section 1.1.

A.2.2 Lemma (TRiangle Inequality for the Hausdorff Semi-Distance). Let $X$ be a metric space and $d$ denote the Hausdorff semi-distance. Then, for all nonempty sets $A, B, C \subset X$, the relation

$$
d(A \mid C) \leq d(A \mid B)+d(B \mid C)
$$

is fulfilled. 
ProOF. Obviously, for all nonempty sets $M_{1}, M_{2} \subset X$, the Hausdorff semi-distance fulfills

$$
d\left(M_{1} \mid M_{2}\right)=\inf \left\{\delta>0: M_{1} \subset U_{\delta}\left(M_{2}\right)\right\} .
$$

Hence, for all $\varepsilon>0$, we have

$$
A \subset U_{d(A \mid B)+\frac{\varepsilon}{2}}(B) \quad \text { and } \quad B \subset U_{d(B \mid C)+\frac{\varepsilon}{2}}(C) .
$$

This implies $A \subset U_{d(A \mid B)+d(B \mid C)+\varepsilon}(C)$ and finishes the proof of this lemma.

A.2.3 Lemma. Let $A, B, C$ be linear subspaces of the $\mathbb{R}^{N}$ such that $A \supset C$. Then, the relation

$$
A \cap(B+C)=(A \cap B)+C
$$

is fulfilled.

Proof. See SiEgmund [170, Hilfssatz 2.36, p. 58].

\section{A.3 Projective Spaces}

In this section, the real projective space $\mathbb{P}^{N-1}$ of the vector space $\mathbb{R}^{N}$ is introduced, and some basic properties are derived. Here, the $\mathbb{R}^{N}$ is equipped with the Euclidian norm $\|\cdot\|$ and the Euclidian scalar product $\langle\cdot, \cdot\rangle$ (cf. Section 1.1). We say, two nonzero elements $x, y \in \mathbb{R}^{N}$ are equivalent if there exists a real number $c \in \mathbb{R}$ such that $x=c y$. The equivalence class of $x \in \mathbb{R}^{N}$ is denoted by $\mathbb{P} x$, and we call the set of all equivalent classes the projective space $\mathbb{P}^{N-1}$. Equipped with the metric $d_{\mathbb{P}}: \mathbb{P}^{N-1} \times \mathbb{P}^{N-1} \rightarrow[0, \sqrt{2}]$, given by

$$
d_{\mathbb{P}}(\mathbb{P} v, \mathbb{P} w)=\min \left\{\left\|\frac{v}{\|v\|}-\frac{w}{\|w\|}\right\|,\left\|\frac{v}{\|v\|}+\frac{w}{\|w\|}\right\|\right\} \quad \text { for all } v, w \in \mathbb{R}^{N},
$$

the projective space is a compact metric space. For any $v \in \mathbb{P}^{N-1}$, we define

$$
\mathbb{P}^{-1} v:=\left\{x \in \mathbb{R}^{N}: \mathbb{P} x=v\right\} \cup\{0\} .
$$

A.3.1 LemmA. For all $\varepsilon>0$, there exists a $\delta \in(0,1)$ such that for all nonzero $v, w \in \mathbb{R}^{N}$ with

$$
\frac{\langle v, w\rangle^{2}}{\|v\|^{2}\|w\|^{2}} \geq 1-\delta
$$

we have

$$
d_{\mathbb{P}}(\mathbb{P} v, \mathbb{P} w) \leq \varepsilon .
$$

Proof. This is a direct consequence of Colonius \& KLIEMAnN [49, Lemma B.1.17., p. 538].

A.3.2 LemmA. Let $V, W \subset \mathbb{R}^{N}$ be linear subspaces of the $\mathbb{R}^{N}$ with $V \subsetneq W$. Then,

$$
d_{\mathbb{P}}(\mathbb{P} W \mid \mathbb{P} V)=\sqrt{2}
$$


Proof. The linear subspace $V^{\perp} \cap W$, where $V^{\perp}:=\left\{x \in \mathbb{R}^{N}:\langle x, v\rangle=0\right.$ for all $\left.v \in V\right\}$, is obviously nontrivial. Let $w$ be a nonzero element of $V^{\perp} \cap W$. Then, for all $v \in V$, we have

$$
\begin{aligned}
& d_{\mathbb{P}}(\mathbb{P} w, \mathbb{P} v)=\min \left\{\left\|\frac{v}{\|v\|} \pm \frac{w}{\|w\|}\right\|\right\} \\
& =\min \left\{\sqrt{(\underbrace{\left\langle\frac{v}{\|v\|}, \frac{v}{\|v\|}\right\rangle}_{=1}+\underbrace{\left\langle\frac{w}{\|w\|}, \frac{w}{\|w\|}\right\rangle}_{=1} \pm 2\langle\underbrace{\left\langle\frac{v}{\|v\|}, \frac{w}{\|w\|}\right)}_{=0}\}}=\sqrt{2} .\right.
\end{aligned}
$$

Since $d_{\mathbb{P}}(x, y) \leq \sqrt{2}$ for all $x, y \in \mathbb{P}^{N-1}$, this implies the assertion. 



\section{BIBLIOGRAPHY}

[1] Abraham, R. H., Marsden, J. E., And Ratiu, T. Manifolds, Tensor Analysis, and Applications. Springer, New York, 1988.

[2] Agarwal, R. P. Difference Equations and Inequalities. Marcel Dekker Inc., New York, 1992.

[3] AkIn, E. The General Topology of Dynamical Systems. No. 1 in Graduate Studies in Mathematics. American Mathematical Society, Providence, Rhode Island, 1993.

[4] Andronov, A. A., And Pontryagin, L. Systemes grossiers. Dokl. Akad. Nauk SSSR 14 (1937), 247-251.

[5] Arnold, L. Random Dynamical Systems. Springer, Berlin Heidelberg New York, 1998.

[6] ARnold, L. Recent Progress in Stochastic Bifurcation Theory. Report Nr. 439, Institut für Dynamische Systeme, Universität Bremen, 1999.

[7] ARnold, L., And Schmalfuss, B. Lyapunov's Second Method for Random Dynamical Systems. Journal of Differential Equations 177, 1 (2001), 235-265.

[8] Arnold, L., Sri Namachchivaya, N., And Schenk-Hoppé, K. R. Toward an Understanding of Stochastic Hopf Bifurcation: A Case Study. International Journal of Bifurcation and Chaos 6, 11 (1996), 1947-1975.

[9] ARnOld, L., AND XU, K. Invariant Measures for Random Dynamical Systems, and a Necessary Condition for Stochastic Bifurcation from a Fixed Point. Random \& Computational Dynamics 2, 2 (1994), 165-182.

[10] ARtstein, Z. Limiting Equations and Stability of Nonautonomous Ordinary Differential Equations. In J. P. LaSalle, The Stability of Dynamical Systems, vol. 25 of CBMS Regional Conference Series in Applied Mathematics. SIAM, Philadelphia, 1976, pp. 57-76.

[11] Artstein, Z. The Limiting Equations of Nonautonomous Ordinary Differential Equations. Journal of Differential Equations 25 (1977), 184-202.

[12] Aubin, J. P., And Frankowska, H. Set-Valued Analysis, vol. 2 of Systems and Control: Foundations and Applications. Birkhäuser, Boston, 1990.

[13] Aulbach, B. A Reduction Principle for Nonautonomous Differential Equations. Archiv der Mathematik 39 (1982), 217-232. 
[14] AulBach, B. Gewöhnliche Differenzialgleichungen. Spektrum Akademischer Verlag, Heidelberg, 2004. (in German).

[15] Aulbach, B., And Kalkbrenner, J. Exponential Forward Splitting for Noninvertible Difference Equations. Computers \& Mathematics with Applications 42 (2001), 743-754.

[16] Aulbach, B., Rasmussen, M., And Siegmund, S. Approximation of Attractors of Nonautonomous Dynamical Systems. Discrete and Continuous Dynamical Systems B 5, 2 (2005), 215-238.

[17] Aulbach, B., Rasmussen, M., And Siegmund, S. Invariant Manifolds as Pullback Attractors of Nonautonomous Difference Equations. In Proceedings of the Eighth International Conference on Difference Equations and Applications (Boca Raton, 2005), S. Elaydi, G. Ladas, B. Aulbach, and O. Dosly, Eds., CRC Press.

[18] Aulbach, B., Rasmussen, M., And Siegmund, S. Invariant Manifolds as Pullback Attractors of Nonautonomous Differential Equations. Discrete and Continuous Dynamical Systems 15, 2 (2006), 579-596.

[19] Aulbach, B., And Siegmund, S. A Spectral Theory for Nonautonomous Difference Equations. In Proceedings of the Fifth Conference on Difference Equations and Applications, Tетисо/Chile 2000 (2000), Gordon \& Breach Publishers.

[20] Aulbach, B., And Siegmund, S. The Dichotomy Spectrum for Noninvertible Systems of Linear Difference Equations. Journal of Difference Equations and Applications 7, 6 (2001), 895-913.

[21] Aulbach, B., And Wanner, T. Integral Manifolds for Carathéodory Type Differential Equations in Banach Spaces. In Six Lectures on Dynamical Systems, B. Aulbach and F. Colonius, Eds. World Scientific, Singapore, 1996.

[22] Aulbach, B., And Wanner, T. Invariant Foliations for Carathéodory Type Differential Equations in Banach Spaces. In Advances of Stability Theory at the End of XX Century, V. Lakshmikantham and A. A. Martynyuk, Eds. Gordon \& Breach Publishers, 1999.

[23] Auslander, J., Bhatia, N. P., And Seibert, P. Attractors in Dynamical Systems. Boletín de la Sociedad Matemática Mexicana. Segunda Serie. 9 (1964), 55-66.

[24] Barreira, L., And Pesin, Y. B. Lyapunov Exponents and Smooth Ergodic Theory, vol. 23 of University Lecture Series. American Mathematical Society, Providence, Rhode Island, 2002.

[25] Baxendale, P. Wiener Processes on Manifolds of Maps. Proceedings of the Royal Society of Edinburgh. Section A 87 (1980), 127-152.

[26] Bebutov, M. V. Sur les systèmes dynamiques dans l'espace des fonctions continues. C. R. Acad. Sci. URSS 27 (1940), 904-906.

[27] Benoît, E., Ed. Dynamic Bifurcations, vol. 1493 of Springer Lecture Notes in Mathematics. Springer, Berlin, 1991. 
[28] Berger, A., And Siegmund, S. On the Gap between Random Dynamical Systems and Continuous Skew Products. Journal of Dynamics and Differential Equations 15, 2-3 (2003), 237-279.

[29] Berglund, N. Adiabatic Dynamical Systems and Hysteresis. Thesis EPFL no. 1800, 1998.

[30] Bhatia, N. P., And Szegö, G. P. Stability Theory of Dynamical Systems. Springer, Berlin Heidelberg New York, 1970.

[31] Birkhoff, G. D. Dynamical Systems, vol. 9 of Colloquium Publications. American Mathematical Society, New York, 1927.

[32] Bismut, J.-M. A Generalized Formula of Itô and some other Properties of Stochastic Flows. Zeitschrift für Wahrscheinlichkeitstheorie und verwandte Gebiete 55 (1981), 331350 .

[33] Braaksma, B. L. J., And Broer, H. W. On a Quasi-Periodic Hopf Bifurcation. Annales de l'Institut Henri Poincaré - Analyse Non Linéaire 4, 2 (1987), 115-168.

[34] Braga Barros, C. J., And San Martin, L. A. B. Chain Transitive Sets for Flows on Flag Bundles. to appear in: Forum Mathematicum.

[35] Broer, H. W. Quasi Periodicity in Local Bifurcation Theory. In Bifurcation Theory, Mechanics and Physics (1983), C. P. Bruter, A. Aragnol, and A. Lichnerowicz, Eds., D. Reidel Publishing Company, pp. 177-208.

[36] Broer, H. W., Huitema, G. B., Takens, F., and Braaksma, B. L. J. Unfoldings and Bifurcations of Quasi-Periodic Tori. No. 421 in Memoirs of the AMS. American Mathematical Society, Providence, Rhode Island, 1990.

[37] Caraballo, T., And Langa, J. A. On the Upper Semicontinuity of Cocycle Attractors for Non-Autonomous and Random Dynamical Systems. Dynamics of Continuous, Discrete and Impulsive Systems A 10, 4 (2003), 491-513.

[38] CARr, J. Applications of Centre Manifold Theory, vol. 35 of Applied Mathematical Sciences. Springer, Berlin Heidelberg New York, 1981.

[39] Cesari, L. Asymptotic Behavior and Stability Problems in Ordinary Differential Equations. Springer, Berlin, 1963.

[40] Cheban, D. N., Kloeden, P. E., And Schmalfuss, B. Pullback Attractors in Dissipative Nonautonomous Differential Equations Under Discretization. Journal of Dynamics and Differential Equations 13, 1 (2001), 185-213.

[41] Cheban, D. N., Kloeden, P. E., And Schmalfuss, B. The Relationship between Pullback, Forward and Global Attractors of Nonautonomous Dynamical Systems. Nonlinear Dynamics and Systems Theory 2, 2 (2002), 125-144.

[42] Chenciner, A., And Iooss, G. Bifurcations de tores invariants. Archive for Rational Mechanics and Analysis 69 (1979), 109-198. 
[43] Chenciner, A., And Iooss, G. Persistance et bifurcation de tores invariants. Archive for Rational Mechanics and Analysis 71 (1979), 301-306.

[44] Chicone, C. Ordinary Differential Equations with Applications, vol. 34 of Texts in Applied Mathematics. Springer, New York, 1999.

[45] Chow, S.-N., And Hale, J. K. Methods of Bifurcation Theory, vol. 251 of Grundlehren der mathematischen Wissenschaften. Springer, Berlin Heidelberg New York, 1996.

[46] Chow, S.-N., Li, C., AND WANG, D. Normal Forms and Bifurcation of Planar Vector Fields. Cambridge University Press, 1994.

[47] Coddington, E. A., And Levinson, N. Theory of Ordinary Differential Equations. McGraw-Hill Book Company, New York Toronto London, 1955.

[48] Colonius, F., And Kliemann, W. The Morse Spectrum of Linear Flows on Vector Bundles. Transactions of the American Mathematical Society 348, 11 (1996), 4355-4388.

[49] Colonius, F., And Kliemann, W. The Dynamics of Control. Birkhäuser, 2000.

[50] Colonius, F., And Kliemann, W. Morse Decompositions and Spectra on Flag Bundles. Journal of Dynamics and Differential Equations 14, 4 (2002), 719-741.

[51] Colonius, F., Kloeden, P. E., And Siegmund, S., Eds. Foundations of Nonautonomous Dynamical Systems (2004), Special Issue of Stochastics and Dynamics 4, 3.

[52] Conley, C. C. Isolated Invariant Sets and the Morse Index. No. 38 in Regional Conference Series in Mathematics. American Mathematical Society, Providence, Rhode Island, 1978.

[53] Coppel, W. A. Stability and Asymptotic Behavior of Differential Equations. Heath, Boston, 1965.

[54] Coppel, W. A. Dichotomies in Stability Theory, vol. 629 of Springer Lecture Notes in Mathematics. Springer, Berlin Heidelberg New York, 1978.

[55] Crauel, H., Debussche, A., And Flandoli, F. Random Attractors. Journal of Dynamics and Differential Equations 9, 2 (1997), 307-341.

[56] Crauel, H., Duc, L. H., And Siegmund, S. Towards a Morse Theory for Random Dynamical Systems. Stochastics and Dynamics 4, 3 (2004), 277-296.

[57] Crauel, H., And Flandoli, F. Attractors for Random Dynamical Systems. Probability Theory and Related Fields 100, 3 (1994), 365-393.

[58] Crauel, H., Imkeller, P., And Steinkamp, M. Bifurcation of One-Dimensional Stochastic Differential Equation. In Stochastic Dynamics, H. Crauel and M. Gundlach, Eds. Springer, Berlin Heidelberg New York, 1999, pp. 27-47.

[59] CRAWFORD, J. Introduction to Bifurcation Theory. Reviews of Modern Physics 63 (1991), 991-1037. 
[60] DaleckiĬ, J. L., AND KReǏn, M. G. Stability of Solutions of Differential Equations in Banach Spaces, vol. 43 of Translations of Mathematical Monographs. American Mathematical Society, Providence, Rhode Island, 1974.

[61] Dieci, L., AND VAn Vleck, E. S. Lyapunov and Other Spectra: A Survey. In Collected Lectures on the Preservation of Stability under Discretization. SIAM, 2002, pp. 197-218.

[62] Dieci, L., AND VAn Vleck, E. S. Lyapunov Spectral Intervals: Theory and Computation. SIAM Journal on Numerical Analysis 40, 2 (2002), 516-542.

[63] Elstrodt, J. Maß- und Integrationstheorie. Springer, Berlin Heidelberg New York, 1996. (in German).

[64] Elworthy, K. D. Stochastic Dynamical Systems and Their Flows. In Stochastic Analysis (New York, 1978), A. Friedman and M. Pinsky, Eds., Academic Press, pp. 79-95.

[65] Fabbri, R., And Johnson, R. A. On a Saddle-Node Bifurcation in a Problem of QuasiPeriodic Harmonic Forcing. Preprint, 2005.

[66] Fabbri, R., Johnson, R. A., And Mantellini, F. A Nonautonomous Saddle-Node Bifurcation Pattern. Stochastics and Dynamics 4, 3 (2004), 335-350.

[67] Flandoli, F., And Schmalfuss, B. Random Attractors for the 3-D Stochastic NavierStokes Equation with Mulitiplicative White Noise. Stochastics and Stochastics Reports 59, 1-2 (1996), 21-45.

[68] Franks, J. A Variation on the Poincaré-Birkhoff Theorem. In Hamiltonian Dynamical Systems (1988), vol. 81 of Contemporary Mathematics, pp. 111-117.

[69] Glendinning, P. Non-Smooth Pitchfork Bifurcations. Discrete and Continuous Dynamical Systems B 4, 2 (2004), 457-464.

[70] Gronwall, T. H. Note on the Derivatives with respect to a Parameter of the Solutions of a System of Differential Equations. Annals of Mathematics 20, 2 (1919), 292-296.

[71] Guckenheimer, J., And Holmes, P. Nonlinear Oscillation, Dynamical Systems, and Bifurcations of Vector Fields. Springer, New York, 1983.

[72] Hadamard, J. Sur l'itération et les solutions asymptotiques des équations différentielles. Bulletin de la Société Mathématique de France 29 (1901), 224-228.

[73] Hahn, W. Stability of Motion. Springer, Berlin, 1967.

[74] Hale, J. K. Topics in Dynamic Bifurcation Theory. No. 47 in Regional Conference Series in Mathematics. American Mathematical Society, Providence, Rhode Island, 1981.

[75] Hale, J. K. Introduction to Dynamic Bifurcation. In Bifurcation Theory and Applications, vol. 1057 of Springer Lecture Notes in Mathematics. Springer, Berlin Heidelberg New York, 1984, pp. 106-151.

[76] Hale, J. K. Asymptotic Behavior of Dissipative Systems, vol. 25 of Mathematical Surveys and Monographs. American Mathematical Society, Providence, Rhode Island, 1988. 
[77] Hale, J. K., AND KoçAK, H. Dynamics and Bifurcations. Springer, New York, 1991.

[78] Henry, D. Geometric Theory of Semilinear Parabolic Equations, vol. 840 of Springer Lecture Notes in Mathematics. Springer, Berlin Heidelberg New York, 1981.

[79] Hirsch, M. W., Pugh, C. C., And Shub, M. Invariant Manifolds, vol. 583 of Springer Lecture Notes in Mathematics. Springer, Berlin Heidelberg New York, 1977.

[80] Hirsch, M. W., And Smale, S. Differential Equations, Dynamical Systems, and Linear Algebra. Pure and Applied Mathematics. Academic Press, 1974.

[81] Holmes, P., And RAnd, D. Phase Portraits and Bifurcations of the Nonlinear Oscillator $\ddot{x}+\left(\alpha+\gamma x^{2}\right) \dot{x}+\beta x+\delta x^{3}=0$. International Journal of Non-Linear Mechanics 15 (1980), 449-458.

[82] Ikeda, N., And Watanabe, S. Stochastic Differential Equations and Diffusion Processes. North Holland-Kodansha, Tokyo, 1981.

[83] Johnson, R. A. An Application of Topological Dynamics to Bifurcation Theory. In Topological Dynamics and Applications. A volume in honor of Robert Ellis, M. G. Nerurkar, D. P. Dokken, and D. B. Ellis, Eds., vol. 215 of Contemporary Mathematics. American Mathematical Society, Providence, Rhode Island, 1998, pp. 323-334.

[84] Johnson, R. A., Kloeden, P. E., And Pavani, R. Two-Step Transition in Nonautonomous Bifurcations: An Explanation. Stochastics and Dynamics 2, 1 (2002), 67-92.

[85] Johnson, R. A., And Mantellini, F. A Nonautonomous Transcritical Bifurcation Problem with an Application to Quasi-Periodic Bubbles. Discrete and Continuous Dynamical Systems 9, 1 (2003), 209-224.

[86] Johnson, R. A., And Yi, Y. Hopf Bifurcation from Non-Periodic Solutions of Differential Equations, II. Journal of Differential Equations 107, 2 (1994), 310-340.

[87] Kalkbrenner, J. Nichthyperbolische exponentielle Dichotomie. Diploma Thesis, University of Augsburg, 1992. (in German).

[88] Kalkbrenner, J. Exponentielle Dichotomie und chaotische Dynamik nichtinvertierbarer Differenzengleichungen. Ph. D. Thesis, University of Augsburg, 1994. (in German).

[89] Kato, J., Martynyuk, A. A., And Shestakov, A. A. Stability of Motion of Nonautonomous Systems (Method of Limiting Equations), vol. 3 of Stability and Control: Theory, Methods and Applications. Gordon \& Breach Publishers, Philadelphia, 1996.

[90] Kelley, A. Stability of the Center-Stable Manifold. Journal of Mathematical Analysis and Applications 18 (1967), 336-344.

[91] Kelley, A. The Stable, Center-Stable, Center, Center-Unstable, Unstable Manifolds. Journal of Differential Equations 3 (1967), 546-570.

[92] Kirchgraber, U., And Palmer, K. J. Geometry in the Neigborhood of Invariant Manifolds of Maps and Flows and Linearization, vol. 233 of Pitman Research Notes in Mathematical Series. Longman, Burnt Mill, 1990. 
[93] Kloeden, P. E. Lyapunov Functions for Cocycle Attractors in Nonautonomous Difference Equations. Izvetsiya Akad Nauk Rep Moldovia Mathematika 26 (1998), 32-42.

[94] Kloeden, P. E. A Lyapunov Function for Pullback Attractors of Nonautonomous Differential Equations. In Conference 05 (2000), Electronic Journal of Differential Equations, pp. 91-102.

[95] Kloeden, P. E. Pullback Attractors in Nonautonomous Difference Equations. Journal of Difference Equations and Applications 6, 1 (2000), 91-102.

[96] Kloeden, P. E. Pitchfork and Transcritical Bifurcations in Systems with Homogeneous Nonlinearities and an Almost Periodic Time Coefficient. Communications on Pure and Applied Analysis 3, 2 (2004), 161-173.

[97] Kloeden, P. E., Keller, H., And Schmalfuss, B. Towards a Theory of Random Numerical Dynamics. In Stochastic Dynamics, H. Crauel and M. Gundlach, Eds. Springer, Berlin Heidelberg New York, 1999.

[98] Kloeden, P. E., And Siegmund, S. Bifurcations and Continuous Transitions of Attractors in Autonomous and Nonautonomous Systems. International Journal of Bifurcation and Chaos 15, 3 (2005), 743-762.

[99] Kunita, H. On the Decomposition of Solutions of Stochastic Differential Equations. In Stochastic Integrals (1981), D. Williams, Ed., vol. 851 of Springer Lecture Notes in Mathematics, pp. 213-255.

[100] KuZnetsow, Y. A. Elements of Applied Bifurcation Theory, vol. 112 of Applied Mathematical Sciences. Springer, New York, 1995.

[101] LANG, S. Real and Functional Analysis. Springer, New York, 1993.

[102] Langa, J. A., Robinson, J. C., And SuÁrez, A. Stability, Instability and Bifurcation Phenomena in Non-Autonomous Differential Equations. Nonlinearity 15, 3 (2002), 887903.

[103] Langa, J. A., Robinson, J. C., And SuÁrez, A. Forwards and Pullback Behaviour of a Non-Autonomous Lotka-Volterra System. Nonlinearity 16, 4 (2003), 1277-1293.

[104] Langa, J. A., Robinson, J. C., And SuÁrez, A. Bifurcations in Non-Autonomous Scalar Equations. Journal of Differential Equations 221, 1 (2006), 1-35.

[105] Lebovitz, N. R., And SchaAR, R. J. Exchange of Stabilities in Autonomous Systems. Studies in Applied Mathematics 54 (1975), 229-260.

[106] Lebovitz, N. R., AND SchaAR, R. J. Exchange of Stabilities in Autonomous Systems II, Vertical Bifurcation. Studies in Applied Mathematics 56 (1977), 1-50.

[107] Lorenz, E. N. Deterministic Nonperiodic Flow. Journal of the Atmospheric Sciences 20 (1963), 130-141.

[108] Luo, Wang, Zhu, And Han. Bifurcation Theory and Methods of Dynamical Systems. World Scientific, Singapore, 1997. 
[109] Lyapunov, A. M. The General Problem of the Stability of Motion. Mathematical Society of Kharkov, Kharkov, 1892. (in Russian).

[110] Lyapunov, A. M. Sur les figures d'equilibre peu differentes des ellipsodies d'une masse liquide homogène donnee d'un mouvement de rotation. Academy of Science St. Petersburg, St. Petersburg, 1906. (in French).

[111] Lyapunov, A. M. Problème générale de la stabilité de mouvement. Annales de la Faculte des Sciences de Toulouse 9 (1907), 203-474. (in French).

[112] Lyapunov, A. M. Stability of Motion, vol. 30 of Mathematics in Science and Engineering. Academic Press, New York, London, 1966. Translated from Russian by F. Abramovici and M. Shimshoni.

[113] Ma, T., And Wang, S. Attractor Bifurcation Theory and its Applications to RayleighBénard Convection. Communications on Pure and Applied Analysis 2, 4 (2003), 591-599.

[114] Markus, L. Asymptotically Autonomous Differential Systems. In Contributions to the Theory of Nonlinear Oscillations III, Annals of Mathematical Studies, S. Lefschetz, Ed., vol. 36. Princeton University Press, 1956, pp. 17-29.

[115] Marsden, J. E., And Hughes, T. J. R. Mathematical Foundations of Elasticity. Prentice-Hall, Englewood Cliffs, New Jersey, 1983.

[116] Marsden, J. E., And McCracken, M. The Hopf Bifurcation and its Applications, vol. 19 of Applied Mathematical Sciences. Springer, Berlin Heidelberg New York, 1976.

[117] Massera, J. L., AND Schäffer, J. J. Linear Differential Equations and Function Spaces. Academic Press, New York, London, 1966.

[118] Mendelson, P. On Unstable Attractors. Boletín de la Sociedad Matemática Mexicana. Segunda Serie. 5 (1960), 270-276.

[119] Miller, R. K. Almost Periodic Differential Equations as Dynamical Systems with Applications to the Existence of Almost Periodic Solutions. Journal of Differential Equations 1, 3 (1965), 337-345.

[120] Mischaikow, K., Smith, H., And Thieme, H. R. Asymptotically Autonomous Semiflows: Chain Recurrence and Lyapunov Functions. Transactions of the American Mathematical Society 347, 5 (1995), 1669-1685.

[121] Norton, D. E. The Fundamental Theorem of Dynamical Systems. Commentationes Mathematicae Universitatis Carolinae 36 (1995), 585-597.

[122] Ochs, G. Weak Random Attractors. Report Nr. 449, Institut für Dynamische Systeme, Universität Bremen, 1999.

[123] Palis, J., And De Melo, W. Geometric Theory of Dynamical Systems. An Introduction. Springer, Berlin Heidelberg New York, 1982.

[124] PAlmer, K. Exponential Dichotomy, Integral Separation and Diagonalizability of Linear Systems of Ordinary Differential Equations. Journal of Differential Equations 43 (1982), 184-203. 
[125] Palmer, K. Exponential Separation, Exponential Dichotomy and Spectral Theory for Linear Systems of Ordinary Differential Equations. Journal of Differential Equations 46 (1982), 324-345.

[126] Palmer, K. Exponential Dichotomies and Transversal Homoclinic Points. Journal of Differential Equations 55 (1984), 225-256.

[127] Palmer, K., And Siegmund, S. Generalized Attractor-Repeller Pairs, Diagonalizability and Integral Separation. Advanced Nonlinear Studies 4 (2004), 189-207.

[128] Papaschinopoulos, G. Exponential Separation, Exponential Dichotomy, and Almost Periodicity of Linear Difference Equations. Journal of Mathematical Analysis and Applications 120 (1986), 276-287.

[129] PERron, O. Über Stabilität und asymptotisches Verhalten der Integrale von Differentialgleichungssystemen. Mathematische Zeitschrift 29 (1928), 129-160.

[130] PERron, O. Die Stabilitätsfrage bei Differentialgleichungen. Mathematische Zeitschrift 32 (1930), 703-728.

[131] Pliss, V. A. Principle Reduction in the Theory of the Stability of Motion. Izv. Akad. Nauk SSSR, Mat. Ser. 28 (1964), 1297-1323. (in Russian).

[132] Pliss, V. A., AND Sell, G. R. Robustness of Exponential Dichotomies in InfiniteDimensional Dynamical Systems. Journal of Dynamics and Differential Equations 11, 3 (1999), 471-513.

[133] Poincaré, H. Sur les propriétés des fonctions définies par les équations aux différences partielles. Thèse, Gauthier-Villars, Paris, 1879. (in French).

[134] Poincaré, H. Sur l'equilibre d'une masse fluids animes d'un mouvement de rotation. Acta Mathematica 7 (1885), 259-380. (in French).

[135] Poincaré, H. Mémoire sur les courbes définie par une équation différentielle IV. Journal de Mathématiques pures et appliquées 2 (1886), 151-217. (in French).

[136] Poincaré, H. Les méthodes nouvelles de la mécanique céleste. Gauthier-Villars, Paris, 1892-1899. (3 volumes, in French).

[137] Pötzsche, C. Extended Hierarchies of Invariant Fiber Bundles for Dynamic Equations on Measure Chains. Preprint.

[138] Pötzsche, C. Exponential Dichotomies of Linear Dynamic Equations on Measure Chains under Slowly Varying Coefficients. Journal of Mathematical Analysis and Applications 289, 1 (2004), 317-335.

[139] Pötzsche, C., And Rasmussen, M. Taylor Approximation of Integral Manifolds. to appear in: Journal of Dynamics and Differential Equations.

[140] Pötzsche, C., And Rasmussen, M. Local Approximation of Invariant Fiber Bundles: An Algorithmic Approach. In Difference Equations and Discrete Dynamical Systems (Singapore, 2005), L. Allen, B. Aulbach, S. Elaydi, and R. Sacker, Eds., World Scientific. 
[141] Pötzsche, C., And Rasmussen, M. Taylor Approximation of Invariant Fiber Bundles of Nonautonomous Difference Equations. Nonlinear Analysis. Theory, Methods \& Applications 60, 7 (2005), 1303-1330.

[142] Rasmussen, M. Morse Decompositions of Nonautonomous Dynamical Systems. to appear in: Transactions of the American Mathematical Society.

[143] Rasmussen, M. Approximation von Attraktoren und Mannigfaltigkeiten nichtautonomer Systeme. Diploma Thesis, University of Augsburg, 2002. (in German).

[144] Rasmussen, M. Towards a Bifurcation Theory for Nonautonomous Difference Equations. Journal of Difference Equations and Applications 12, 3-4 (2006), 297-312.

[145] Robinson, C. Dynamical Systems. Stability, Symbolic Dynamics and Chaos, 2 ed. CRC Press, Boca Raton, 1999.

[146] Ruelle, D. Small Random Perturbations of Dynamical Systems and the Definition of Attractors. Communications in Mathematical Physics 82 (1981), 137-151.

[147] Ruelle, D., And Takens, F. On the nature of turbulence. Communications in Mathematical Physics 20 (1971), 167-192.

[148] Rybakowski, K. P. The Homotopy Index and Partial Differential Equations. Springer, Berlin Heidelberg New York, 1987.

[149] SACKER, R. J. Existence of Dichotomies and Invariant Splittings for Linear Differential Systems IV. Journal of Differential Equations 27 (1978), 106-137.

[150] Sacker, R. J., AND SEll, G. R. Existence of Dichotomies and Invariant Splittings for Linear Differential Systems I. Journal of Differential Equations 15 (1974), 429-458.

[151] SaCKer, R. J., AND SEll, G. R. Existence of Dichotomies and Invariant Splittings for Linear Differential Systems II. Journal of Differential Equations 22 (1976), 478-496.

[152] Sacker, R. J., AND Sell, G. R. Existence of Dichotomies and Invariant Splittings for Linear Differential Systems III. Journal of Differential Equations 22 (1976), 497-522.

[153] Sacker, R. J., And Sell, G. R. A Spectral Theory for Linear Differential Systems. Journal of Differential Equations 27 (1978), 320-358.

[154] Salamon, D., And Zehnder, E. Flows on Vector Bundles and Hyperbolic Sets. Transactions of the American Mathematical Society 306, 2 (1988), 623-649.

[155] SchenK-Hoppé, K. R. Bifurcation Scenarios of the Noisy Duffing-van der Pol Oscillator. Nonlinear Dynamics 11 (1996), 255-274.

[156] SchenK-Hoppé, K. R. Stochastic Hopf Bifurcation: An Example. International Journal of Non-Linear Mechanics 31, 5 (1996), 685-692.

[157] Schenk-Hoppé, K. R. The Stochastic Duffing-Van der Pol Equation. Ph. D. Thesis, Fachbereich Mathematik, Universität Bremen, 1996. 
[158] SchenK-Hoppé, K. R. Random Attractors - General Properties, Existence and Applications to Stochastic Bifurcation Theory. Discrete and Continuous Dynamical Systems 4, 1 (1998), 99-130.

[159] Schmalfuss, B. Backward Cocycles and Attractors of Stochastic Differential Equations. In International Seminar on Applied Mathematics - Nonlinear Dynamics: Attractor Approximation and Global Behaviour (1992), V. Reitmann, T. Riedrich, and N. Koksch, Eds., Technische Universität Dresden, pp. 185-192.

[160] Schmalfuss, B. The Random Attractor of the Stochastic Lorenz System. Zeitschrift für Angewandte Mathematik und Physik 48, 6 (1997), 951-975.

[161] SchmidT, U. Autonome und nichtautonome dynamische Systeme. Diploma Thesis, University of Frankfurt, 2002. (in German).

[162] SchÖNEfuSS, L. W. Nichtautonome Differenzengleichungen und Kettenoperationen. Mitteilungen aus dem Mathematischen Seminar Gießen 207, Gießen, 1992. (in German).

[163] Selgrade, J. F. Isolated Invariant Sets for Flows on Vector Bundles. Transactions of the American Mathematical Society 203 (1975), 359-390.

[164] Sell, G. R. Nonautonomous Differential Equations and Dynamical Systems. I. The Basic Theory. Transactions of the American Mathematical Society 127 (1967), 241-262.

[165] Sell, G. R. Nonautonomous Differential Equations and Dynamical Systems. II. Limiting Equations. Transactions of the American Mathematical Society 127 (1967), 263-283.

[166] Sell, G. R. Topological Dynamics and Ordinary Differential Equations. Van Nostrand Reinhold Mathematical Studies, London, 1971.

[167] SELl, G. R. The Structure of a Flow in the Vicinity of an Almost Periodic Motion. Journal of Differential Equations 27 (1978), 359-393.

[168] SELl, G. R. Bifurcation of Higher Dimensional Tori. Archive for Rational Mechanics and Analysis 69 (1979), 199-230.

[169] Shub, M. Global Stability of Dynamical Systems. Springer, Berlin Heidelberg New York, 1987.

[170] Siegmund, S. Spektral-Theorie, glatte Faserungen und Normalformen für Differentialgleichungen vom Carathéodory-Typ, vol. 30 of Augsburger mathematischnaturwissenschaftliche Schriften. Wißner-Verlag, 1999. (in German).

[171] Siegmund, S. Dichotomy Spectrum for Nonautonomous Differential Equations. Journal of Dynamics and Differential Equations 14, 1 (2002), 243-258.

[172] Siegmund, S. Reducibility of Nonautonomous Linear Differential Equations. Journal of the London Mathematical Society II 65, 2 (2002), 397-410.

[173] SiEnZ, T. Der Attraktorbegriff in dynamischen Systemen. Thesis, University of Augsburg, 2002. (in German). 
[174] Smale, S. Differentiable Dynamical Systems. Bulletin of the American Mathematical Society 73 (1967), 747-817.

[175] Sri Namachchivaya, N. Stochastic Bifurcation. Applied Mathematics and Computation 38,2 (1990), 101-159.

[176] Steinkamp, M. Bifurcations of One-Dimensional Stochastic Differential Equations. Logos Verlag, Berlin, 2000.

[177] Strauss, A., And Yorke, J. A. On Asymptotically Autonomous Differential Equations. Mathematical Systems Theory 1 (1967), 175-182.

[178] Thieme, H. Asymptotically Autonomous Differential Equations in the Plane. Rocky Mountain Journal of Mathematics 24, 1 (1994), 351-380.

[179] Vanderbauwhede, A. Center Manifolds, Normal Forms and Elementary Bifurcations. In Dynamics Reported, U. Kirchgraber and H. O. Walther, Eds., vol. 2. Wiley \& Sons, B. G. Teubner, Stuttgart, 1989, pp. 89-169.

[180] WANnER, T. Invariante Faserbündel und topologische Äquivalenz bei dynamischen Prozessen. Diploma Thesis, Universität Augsburg, 1991. (in German).

[181] Wiggins, S. Introduction to Applied Nonlinear Dynamical Systems and Chaos, vol. 2 of Texts in Applied Mathematics. Springer, New York, 1990.

[182] Wiggins, S. Normally Hyperbolic Invariant Manifolds in Dynamical Systems, vol. 105 of Applied Mathematical Sciences. Springer, New York, 1994.

[183] Yı, Y. A Generalized Integral Manifold Theorem. Journal of Differential Equations 102, 1 (1993), 153-187.

[184] Zeeman, E. C. On the Classification of Dynamical Systems. Bulletin of the London Mathematical Society 20, 6 (1988), 545-557.

[185] Zeeman, E. C. Stability of Dynamical Systems. Nonlinearity 1, 1 (1988), 115-155. 